Florida International University FIU Digital Commons

$11-10-2011$

\title{
Electroproduction of hyperons at low momentum transfer
}

Armando R. Acha

Florida International University

DOI: $10.25148 /$ etd.FI13101518

Follow this and additional works at: https://digitalcommons.fiu.edu/etd

Part of the Physics Commons

\section{Recommended Citation}

Acha, Armando R., "Electroproduction of hyperons at low momentum transfer" (2011). FIU Electronic Theses and Dissertations. 1133. https://digitalcommons.fiu.edu/etd/1133

This work is brought to you for free and open access by the University Graduate School at FIU Digital Commons. It has been accepted for inclusion in FIU Electronic Theses and Dissertations by an authorized administrator of FIU Digital Commons. For more information, please contact dcc@fiu.edu. 
FLORIDA INTERNATIONAL UNIVERSITY

Miami, Florida

\section{ELECTROPRODUCTION OF HYPERONS AT LOW \\ MOMENTUM TRANSFER}

A dissertation submitted in partial fulfillment of the

requirements for the degree of

DOCTOR OF PHILOSOPHY

in

PHYSICS

by

Armando R. Acha

2011 
To: Dean Kenneth Furton

College of Arts and Sciences

This dissertation, written by Armando R. Acha, and entitled Electroproduction of Hyperons at Low Momentum Transfer, having been approved in respect to style and intellectual content, is referred to you for judgment.

We have read this dissertation and recommend that it be approved.

- $1 \frac{\text { John J. LeRose }}{\frac{\text { Lesley Northup }}{\text { Brian Raue }}} \frac{\text { Joerg Reinhold }}{\text { Pete E.C. Markowitz, Major Professor }}$

Date of Defense: November 10, 2011

The dissertation of Armando R. Acha is approved.

Dean Kenneth Furton

College of Arts and Sciences

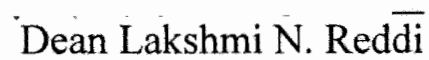

University Graduate School

Florida International University, 2011 
(C) Copyright 2011 by Armando R. Acha All rights reserved. 


\section{DEDICATION}

To my parents Ivanna and Armando, and my dearest wife Thamara. 


\section{ABSTRACT OF THE DISSERTATION \\ ELECTROPRODUCTION OF HYPERONS AT LOW \\ MOMENTUM TRANSFER}

by

Armando R. Acha

Florida International University, 2011

Miami, Florida

Professor Pete E.C. Markowitz, Major Professor

A high resolution study of the $\mathrm{H}\left(e, e^{\prime} K^{+}\right) \Lambda, \Sigma^{0}$ reaction was performed at Hall A, TJNAF as part of the hypernuclear experiment E94-107. One important ingredient to the measurement of the hypernuclear cross section is the elementary cross section for production of hyperons, $\Lambda$ and $\Sigma^{0}$. This reaction was studied using a hydrogen (i.e. a proton) target. Data were taken at very low $Q^{2}\left(\sim 0.07(\mathrm{GeV} / \mathrm{c})^{2}\right)$ and $W \sim 2.2 \mathrm{GeV}$. Kaons were detected along the direction of $\boldsymbol{q}$, the momentum transferred by the incident electron $\left(\theta_{C M} \sim 6^{\circ}\right)$. In addition, there are few data available regarding electroproduction of hyperons at low $Q^{2}$ and $\theta_{C M}$, and the available theoretical models differ significantly in this kinematical region of $W$. The measurement of the elementary cross section was performed by scaling the Monte Carlo cross section (MCEEP) with the experimental-tosimulated yield ratio. The Monte Carlo cross section includes an experimental fit and extrapolation from the existing data for electroproduction of hyperons. Moreover, the estimated transverse component of the electroproduction cross section of $\mathrm{H}\left(e, e^{\prime} K^{+}\right) \Lambda$ was compared to the different predictions of the theoretical models and exisiting data curves for photoproductions of hyperons. None of the models fully describe the cross-section 
results over the entire angular range. Furthermore, measurements of the $\Sigma^{0} / \Lambda$ production ratio were performed at $\theta_{C M} \sim 6^{\circ}$, where data are not available. Finally, data for the measurements of the differential cross sections and the $\Sigma^{0} / \Lambda$ production were binned in $Q^{2}, W$ and $\theta_{C M}$ to understand the dependence on these variables. These results are not only a fundamental contribution to the hypernuclear spectroscopy studies but also an important experimental measurement to constrain existing theoretical models for the elementary reaction. 


\section{TABLE OF CONTENTS}

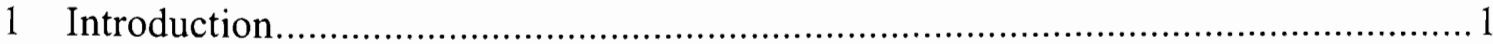

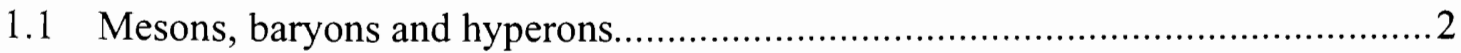

1.2 Hypernuclear physics and the elementary reaction ....................................... 4

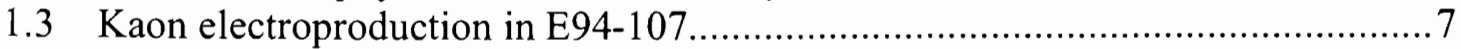

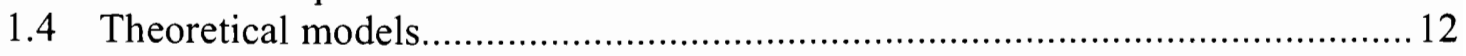

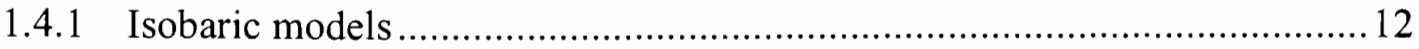

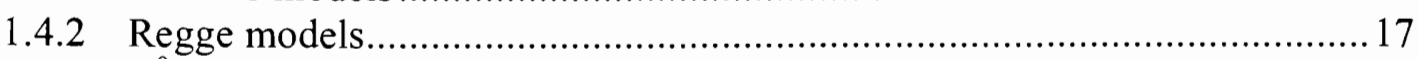

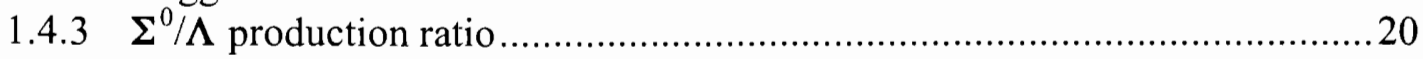

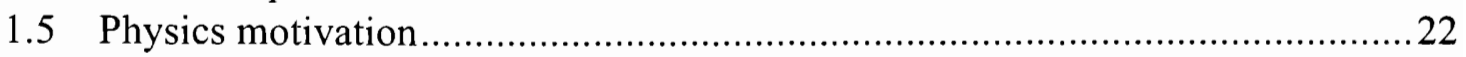

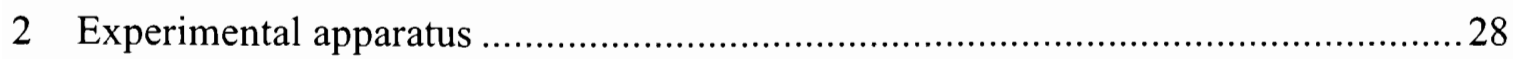

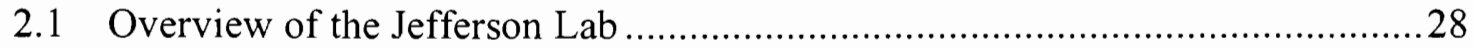

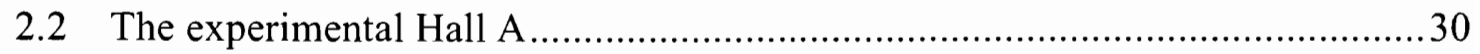

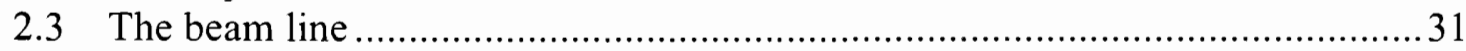

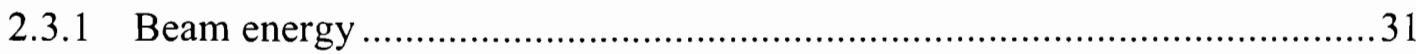

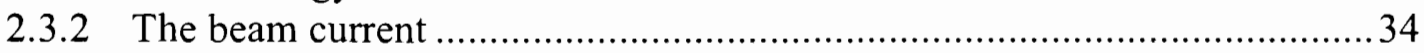

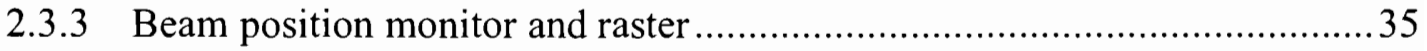

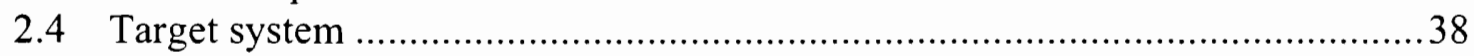

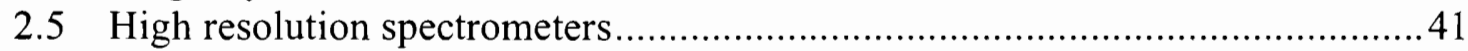

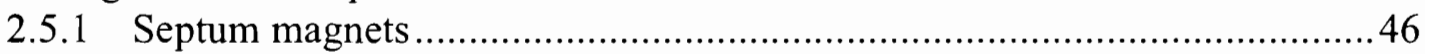

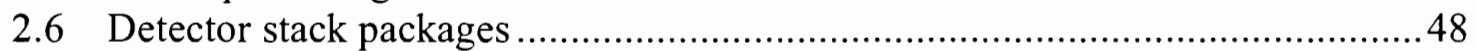

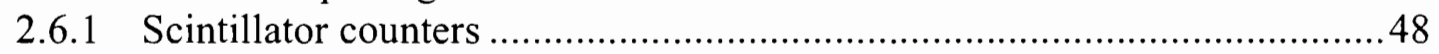

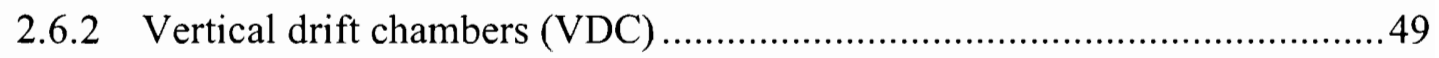

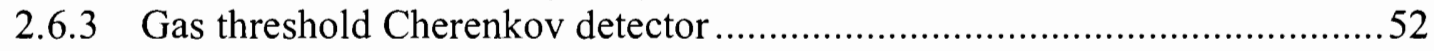

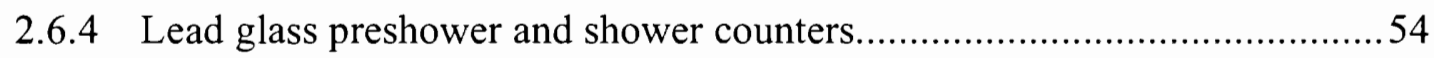

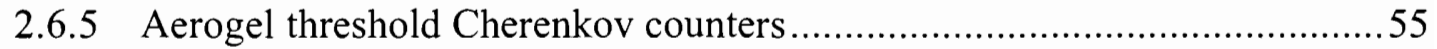

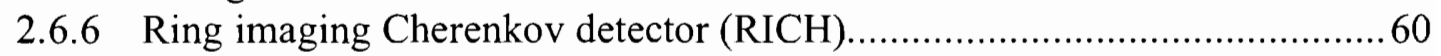

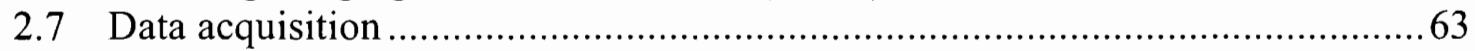

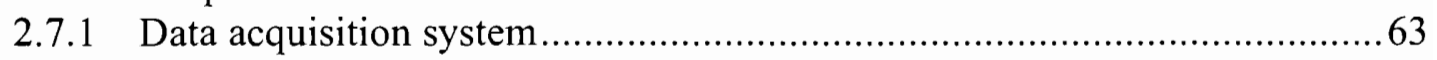

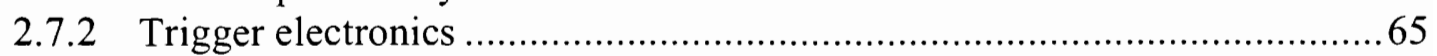

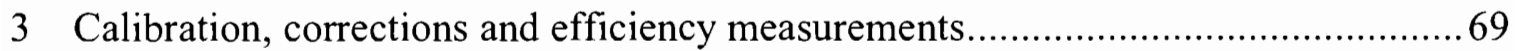

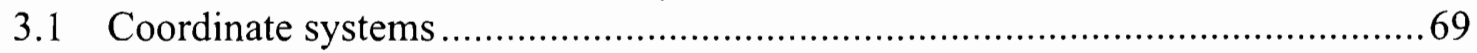

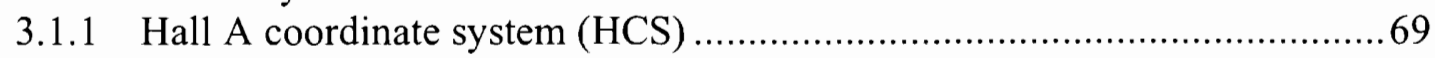

3.1.2 Target coordinate system (TCS) ……................................................... 70

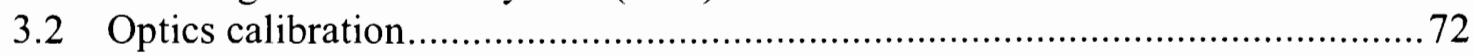

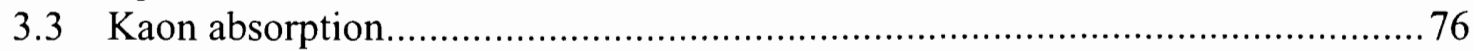

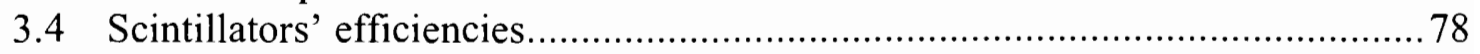

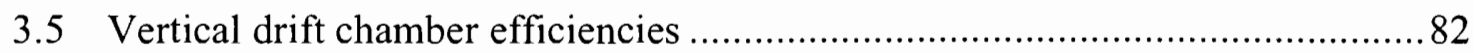

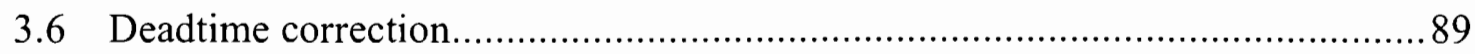

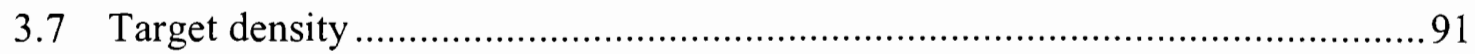




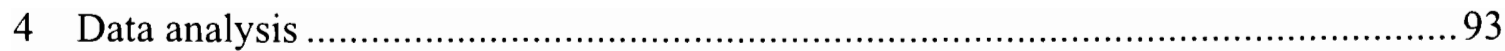

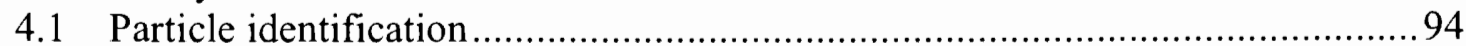

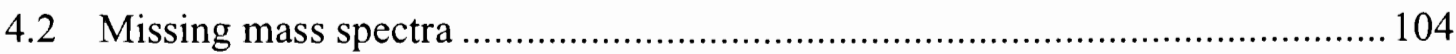

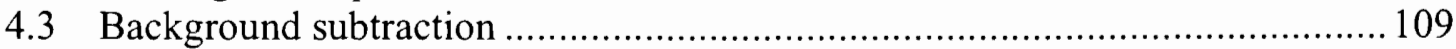

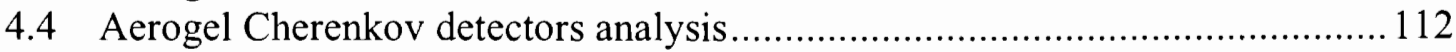

4.5 Gas threshold Cherenkov detector analysis ................................................ 124

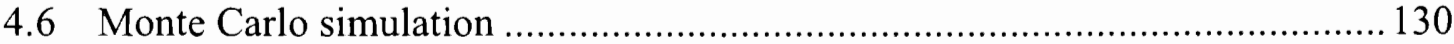

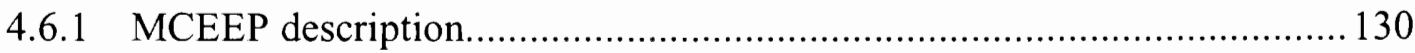

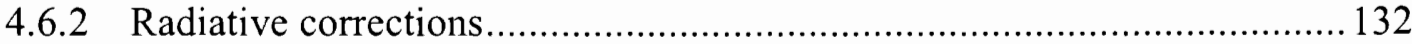

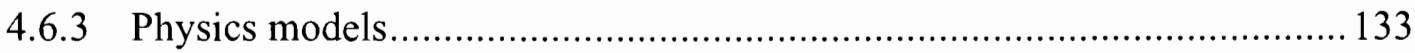

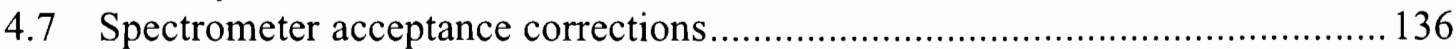

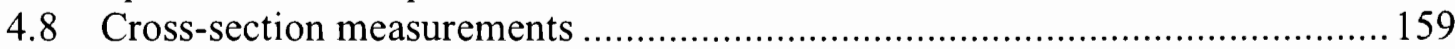

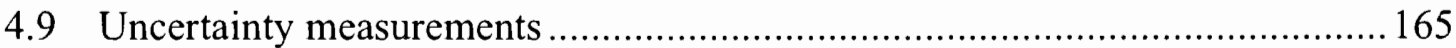

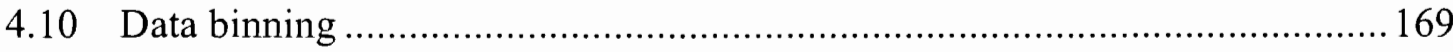

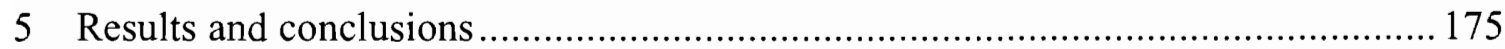

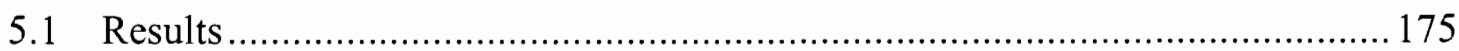

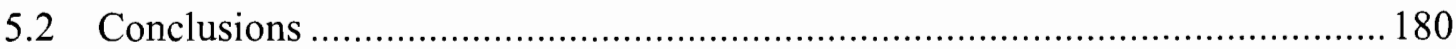

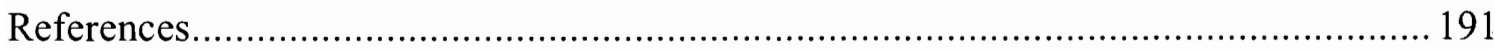

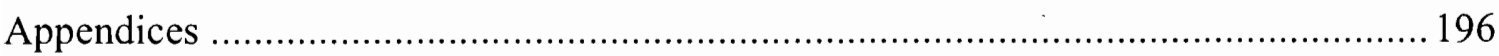

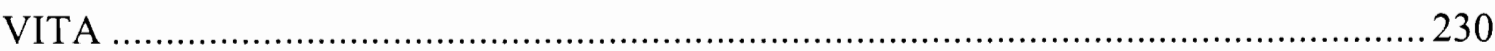




\section{LIST OF TABLES}

TABLE

PAGE

1 Properties of the lightest mesons and baryons [4] ............................................

2 Main properties of the hyperons involved in experiment E94-107 [6]. ....................4

3 Isobaric model coupling constants and resonances for $K \Lambda$ channels [16] ..............15

$4 \quad$ Isobaric model coupling and resonances for $K \boldsymbol{\Sigma}$ channels [16] ........................... 16

5 General characteristics of the HRSs of the Hall A................................................42

6 Hadron arm detector configuration for E94-107 _.................................................43

7 Electron arm detector configuration for E94-107...............................................45

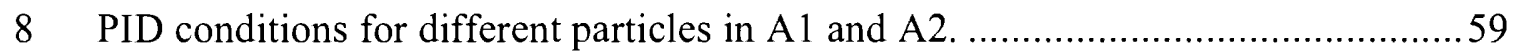

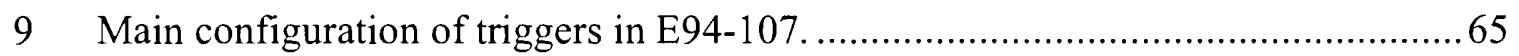

10 Kaon absorption and uncertainties in E94-107............................................... 77

11 Scintillators' efficiencies and uncertainties per run number. ................................ 81

12 VDC efficiencies in E94-107 for the three different kinematics. Firing and tracking

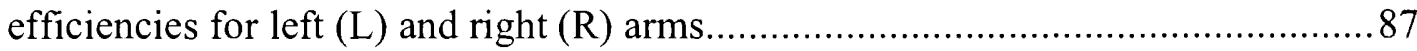

13 VDC efficiency uncertainties in E94-107 for the three different kinematics. Firing and tracking efficiency uncertainties for left $(\mathrm{L})$ and right $(\mathrm{R})$ arms. ....................8 88

14 Livetime (\%) and deadtime correction factors in E94-107 [66].........................90

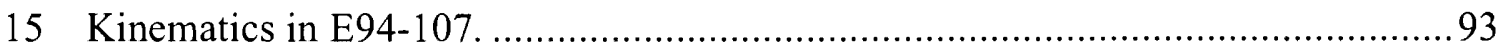

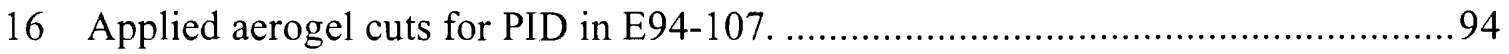

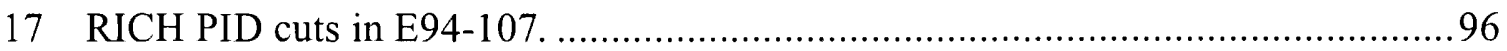

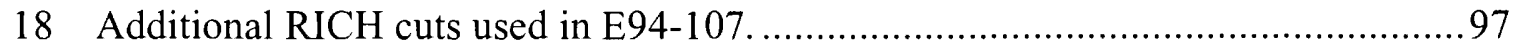

19 Coincidence times for each particle in each kinematics..................................... 104

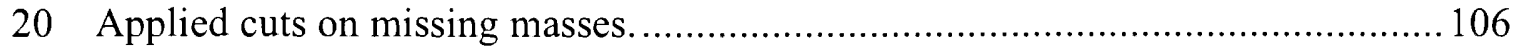


21 Particle/kinematics combinations for analyses. ............................................. 108

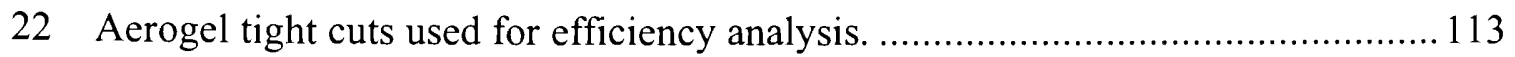

23 Hyperon selection tight cut for the aerogel analysis......................................... 113

24 A1 inefficiency dependence on A1 cut for L_KIN_1...................................... 114

25 A1 inefficiency dependence on A1 cut for L_KIN_2..................................... 115

26 A1 inefficiency dependence on A1 cut for L_KIN_3 ...................................... 116

27 A2 efficiency dependence on A2 cut for L_KIN_1 ........................................ 117

28 A2 efficiency dependence on A2 cut for L_KIN_2 ........................................... 118

29 A2 efficiency dependence on A2 cut for L_KIN_3 ............................................ 119

30 A1 inefficiency dependence on A1 cut for S_KIN_1 ......................................... 120

31 A1 inefficiency dependence on A1 cut for S_KIN_2 ....................................... 121

32 A2 efficiency dependence on A2 cut for S_KIN_1

33 A2 efficiency dependence on A2 cut for S_KIN_2 .......................................... 123

34 Tight cuts applied to A1 and A2 to select clean sample of pions [68].................. 124

$35 \mathrm{CO}_{2}$ gas Cherenkov detector efficiency at different cuts in KIN_1.....................127

$36 \mathrm{CO}_{2}$ gas Cherenkov detector efficiency at different cuts in KIN_2 ..................... 128

$37 \mathrm{CO}_{2}$ gas Cherenkov detector efficiency at different cuts in KIN_3......................129

38 Acceptance variable corrections for all kinematics/particle selections. ................. 144

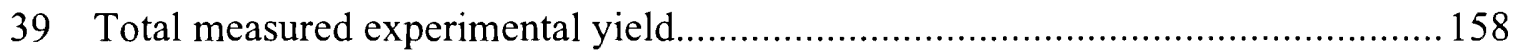

40 Charges and uncertainties in E94-107 from BCM measurements. ....................... 159

41 Correction factors for all kinematics/particles. ............................................. 162

42 KFIT3s.f input values from the MCEEP simulation......................................... 163

43 Expected cross sections for all kinematics/particle selections (from KFIT3s.f)..... 163 
44 Yields and ratios for all kinematics/particle selections. 164

45 Experimental cross sections in E94-107. Experimental average variable values are listed. Uncertainties discussed in Section 4.9. 164

46 Systematic uncertainties from detectors and electronics in the correction factors.. 167

47 Systematic uncertainties from detectors and electronics in the experimental cross sections. 168

48 Uncertainties in target density and integrated beam current................................ 168

49 Statistical uncertainties in the experimental cross sections. .............................. 169

50 Summary of uncertainties in E94-107 (Tables 47, 48 and 49 included). .............. 169

51 Ranges for binning the data in $Q^{2}$ for each kinematics/particle selection. The bins in $Q^{2}$ were labeled; Q1, Q2 and Q3

52 Ranges for binning the data in $W$ for each kinematics/particle selection. The bins in $W$ were labeled; W1, W2 and W3.

53 Ranges for binning the data in $\boldsymbol{\theta}_{C M}$ for each kinematics/particle selection. The bins in $\boldsymbol{\theta}_{C M}$ were labeled; T1, T2 and T3.

54 MCEEP average values for data binning in $Q^{2}$. The bins in $Q^{2}$ were labeled; Q1, Q2 and Q3

55 MCEEP average values for data binning in $W$. The bins in $W$ were labeled; W1, W2 and W3.

56 MCEEP average values for data binning in $\theta_{C M}$. The bins in $\boldsymbol{\theta}_{C M}$ were labeled; T1, $\mathrm{T} 2$ and $\mathrm{T} 3$.

57 Cross-section results for data binning in $Q^{2}$, summed over the other variables. The bins in $Q^{2}$ were labeled; Q1, Q2 and Q3

58 Cross-section results for data binning in $W$, summed over the other variables. The bins in $W$ were labeled; $\mathrm{W} 1, \mathrm{~W} 2$ and $\mathrm{W} 3$.

59 Cross-section results for data binning in $\theta_{C M}$ summed over the other variables. The bins in $\theta_{C M}$ were labeled; T1, T2 and T3 
$60 \frac{\Sigma^{0}}{\Lambda}$ ratios in E94-107.

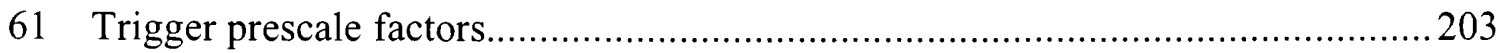

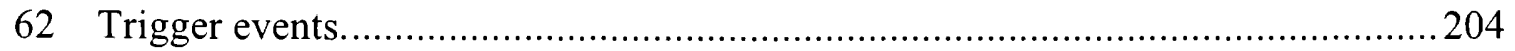

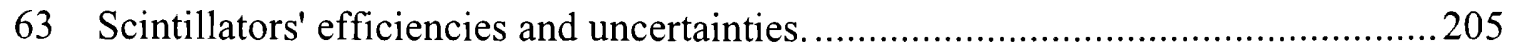

64 VDC firing plane efficiencies for different plane combinations in the left(L) or right

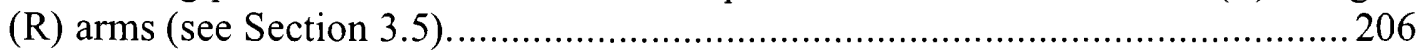

65 VDC single plane number of hits in the left (L) or right $(R)$ arms (see Section 3.5).

66 VDC number of hits for each plane combination indicated in top row in the left (L) or right (R) arms (see Section 3.5).

67 Number of events with specific number of tracks in the the left arm (see Section $3.5)$ 209

68 Number of events with specific number of tracks in the right arm (see Section 3.5).

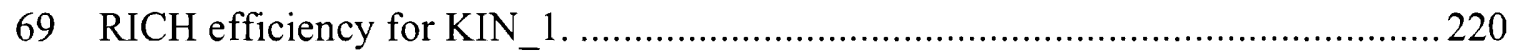

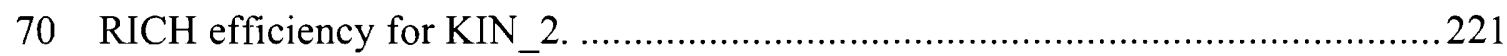

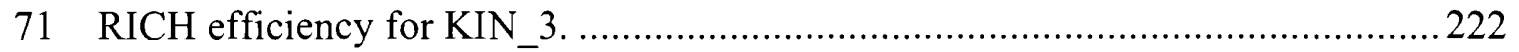

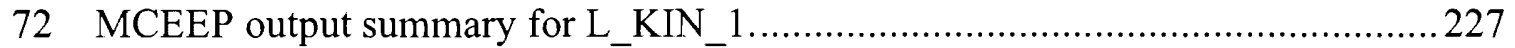

73 MCEEP output summary for L_KIN_2

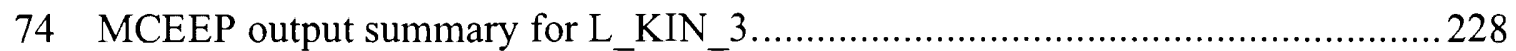

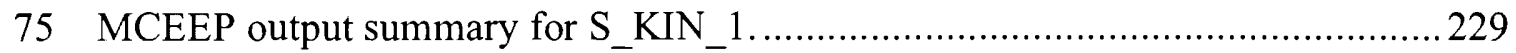

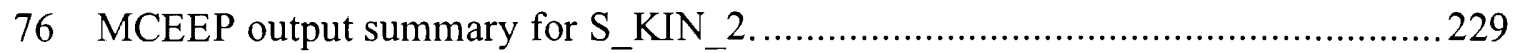




\section{LIST OF FIGURES}

FIGURE

PAGE

1 General view of the kinematics of the elementary reaction.....................................6

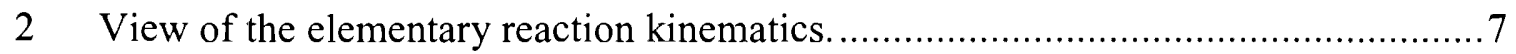

3 Feynman diagramas in isobaric models for kaon electroproduction......................13

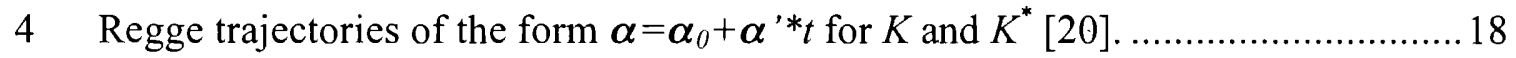

5 World data prior to 1996 for ${ }^{1} \mathrm{H}\left(e, e^{\prime} K^{+}\right) \Lambda,\langle W\rangle=2.15 \mathrm{GeV}, \theta_{C M}=0[11] \ldots \ldots \ldots . .20$

6 World data prior to 1996 for ${ }^{1} \mathrm{H}\left(e, e^{\prime} K^{+}\right) \Sigma^{0},\langle W\rangle=2.15 \mathrm{GeV}, \theta_{C M}=0[11] \ldots \ldots \ldots . .21$

7 Isobaric model predictions compared with experimental data [26].......................23

8 Isobaric and RPR2 models predictions compared to data [26], CLAS data from

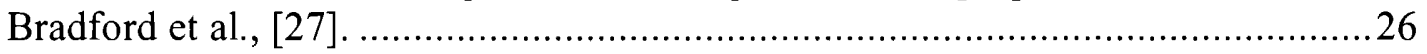

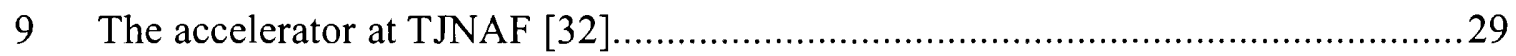

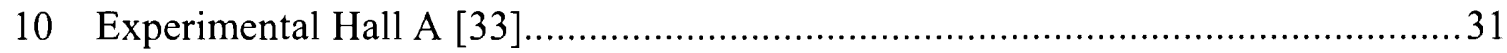

11 Charge ratio of the two amplified signals to the non-amplified signal for experiment E94-107. 35

12 Rastered beam size and position in experiment E94-107.....................................

13 Pictures of the target ladder (left) and cryotarget system (right)..........................38

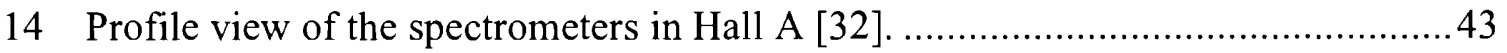

15 Configuration of the detector package in the HRS-L........................................4

16 Configuration of the detector package in the HRS_R .........................................46

17 Picture of one of the septum magnets during fabrication. ..................................4

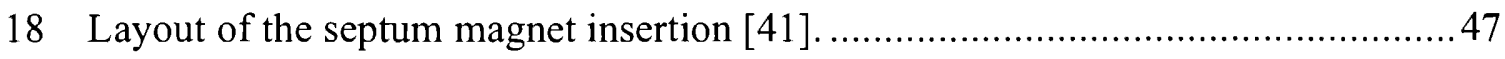

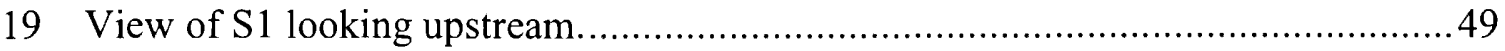




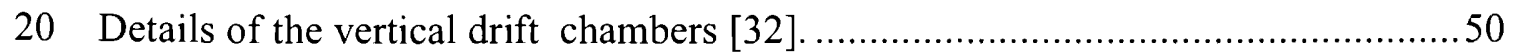

21 Electric field and equipotential lines inside the VDC [43] .................................51

$22 \mathrm{CO}_{2}$ gas threshold Cherenkov detector, JLab PHY98-06..................................53

23 HRS_R shower and preshower counters [32] ..............................................54

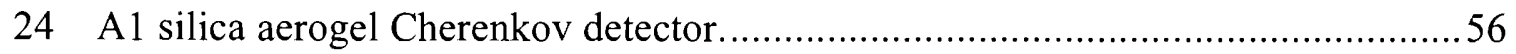

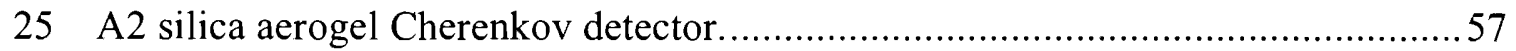

26 Threshold momenta for different particles in A1 and A2 [48] and private

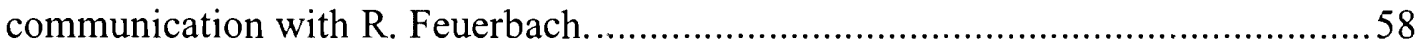

27 Picture showing from left to right; $\mathrm{A} 2, \mathrm{~A} 1$ and $\mathrm{S} 1$, in the hadron arm. Particles traverse the stack from lower right to upper left. .................................................59

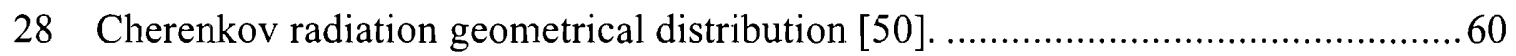

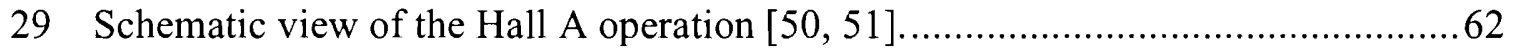

30 Picture of the RICH detector in the HRS-L detector stack, with the A2 just below it.

31 Single arm trigger in electron/hadron spectrometer in Hall A [53] .......................67

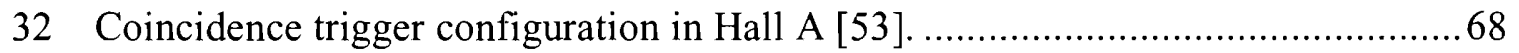

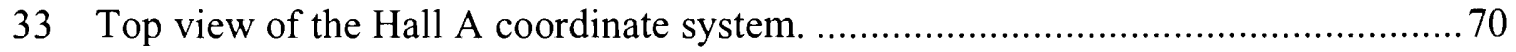

34 Target coordinate system in Hall A (top view) ............................................... 71

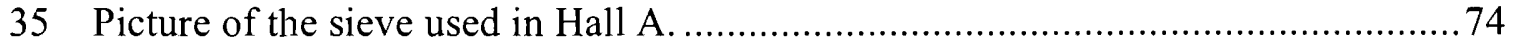

36 Sieve slit design (left), sieve reconstruction, before optimization (central) and after

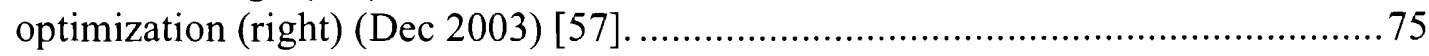

37 Hall A momentum resolution with septum magnets after optics optimization on a thin ${ }^{12} \mathrm{C}$ target (Hall A electronic logbook, December 2003) ...............................76

38 Kaon absorption vs. kaon momentum in E94-107. The errors reflect the $\pm 4 \%$

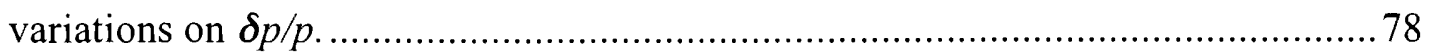

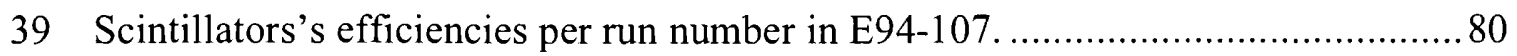


40 Number of hits on the left VDC planes for run 2509. Peak at 5 wires reflects one detected particle, peak at 10 wires reflects two detected particles simultaneously... 84

41 Number of hits on the right VDC planes for run 2509. Peak at 5 wires reflects one detected particle, peak at 10 wires reflects two detected particles simultaneously...86

42 VDC efficiencies in E94-107. .86

43 Livetime in E94-107 per run number. 91

$44 \mathrm{LH}_{2}$ cryotarget density fluctuations vs. beam current, showing three data sets of independent measurements [67].

$45 \mathrm{LH}_{2}$ cryotarget fluctuation vs. raster size, showing three data sets of independent measurents in arbitrary units [67].

46 A1 ADC signal in run 2485 , showing the $1^{\text {st }}, 2^{\text {nd }}$ and $3^{\text {rd }}$ photoelectron peaks, and A1 cut (dashed line) according to Table 16.

47 A2 ADC signal in run 2485 , showing the $1^{\text {st }}$ photoelectron peak, and the two A2 cuts (dashed line) according to Table 16.

$48 \mathrm{CO}_{2}$ gas Cherenkov ADC signal in run 2485, showing "Rcer" PID cut according to Equation 45 to discriminate electrons from negative pions..................................98

49 CT spectrum (ns) for particles identified as kaons (KIN_1) ..................................99

50 CT spectrum (ns) for particles identified as kaons (KIN_2) .............................. 100

51 CT spectrum (ns) for particles identified as kaons (KIN_3) ............................... 100

52 CT spectrum (ns) for particles identified as pions (KIN_1) ................................ 101

53 CT spectrum (ns) for particles identified as pions (KIN_2) ............................... 101

54 CT spectrum (ns) for particles identified as pions (KIN_3) .............................. 102

55 CT spectrum (ns) for particles identified as protons (KIN_1) .............................. 102

56 CT spectrum (ns) for particles identified as protons (KIN_2) …..........................103

57 CT spectrum (ns) for particles identified as protons (KIN_3) .............................. 103

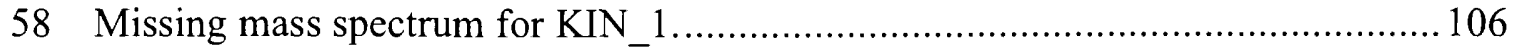




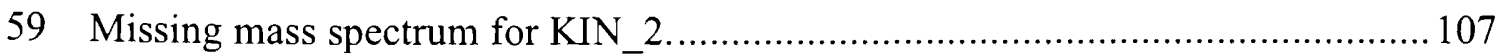

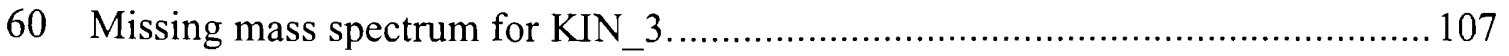

61 Background substracted spectrum for KIN_1

62 Background substracted spectrum for KIN_2 2................................................ 110

63 Background substracted spectrum for KIN_3 ................................................ 111

64 A1 inefficiency dependence on A1 cut for L_KIN_1........................................ 114

65 A1 inefficiency dependence on A1 cut for L_KIN_2........................................ 115

66 A1 inefficiency dependence on A1 cut for L_KIN_3........................................ 116

67 A2 efficiency dependence on A2 cut for L_KIN_1 ........................................... 117

68 A2 efficiency dependence on A2 cut for L_KIN_2 ............................................. 118

69 A2 efficiency dependence on A2 cut for L_KIN_3 .......................................... 119

70 A1 inefficiency dependence on A1 cut for S_KIN_1

71 A1 inefficiency dependence on A1 cut for S_KIN_2 ....................................... 121

72 A2 efficiency dependence on A2 cut for S_KIN_1

73 A2 efficiency dependence on A2 cut for S_KIN_2 ......................................... 123

74 "Banana" plot for KIN_1, showing shower and preshower detector cuts. ............ 125

75 "Banana" plot for KIN_2, showing shower and preshower detector cuts. .............. 126

76 "Banana" plot for KIN_3, showing shower and preshower detector cuts. .............. 126

$77 \mathrm{CO}_{2}$ gas Cherenkov detector efficiency at different cuts in KIN_1.....................127

$78 \mathrm{CO}_{2}$ gas Cherenkov detector efficiency at different cuts in KIN_2 ..................... 128

$79 \mathrm{CO}_{2}$ gas Cherenkov detector efficiency at different cuts in KIN_3..................... 129

80 Cross sections as functions of $W$ with different scaling functions, $f(W)$, overlaid [75] 
81 Yields comparison for L_KIN_1 before corrections (description: page 138) ........139

82 Yields comparison for L_KIN_2 before corrections (description: page 138)........ 140

83 Yields comparison for L_KIN_3 before corrections (description: page 138)........ 141

84 Yields comparison for S_KIN_1 before corrections (description: page 138). ....... 142

85 Yields comparison for S_KIN_2 before corrections (description: page 138)........ 143

86 Yields comparison for L_KIN_1 after corrections (description: page 138).......... 145

87 Yields comparison for L_KIN_2 after corrections (description: page 138)........... 146

88 Yields comparison for L_KIN_3 after corrections (description: page 138)........... 147

89 Yields comparison for S_KIN_1 after corrections (description: page 138).......... 148

90 Yields comparison for S_KIN_2 after corrections (description: page 138)........... 149

91 More yields comparison for L_KIN_1 after corrections (see page 138)................ 151

92 More yields comparison for L_KIN_2 after corrections (see page 138) ................ 152

93 More yields corrections for L_KIN_3 after corrections (see page 138)............... 153

94 More yields comparison for S_KIN_1 after corrections (see page 138)............... 154

95 More yields comparison for S_KIN_2 after corrections (see page 138)...............155

96 Yields distributions on $\varphi$ for L_KIN_1 after corrections. For a description see page

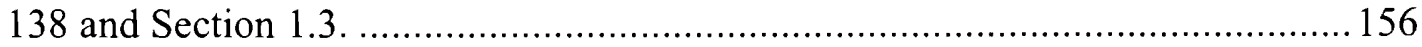

97 Yields distributions on $\varphi$ for L_KIN_2 after corrections. For a description see page 138 and Section 1.3. 156

98 Yields distributions on $\varphi$ for $\mathrm{L}_{-} \mathrm{KIN}_{-} 3$ after corrections. For a description see page 138 and Section 1.3.

99 Yields distributions on $\varphi$ for S_KIN_1 after corrections. For a description see page 138 and Section 1.3

100 Yields distributions on $\varphi$ for S_KIN_2 after corrections. For a description see page 138 and Section 1.3. 
$102 \Lambda$-channel measured cross section vs. average kaon momentum for unbinned data. $W=2.2 \mathrm{GeV}, Q^{2} \sim 0.07(\mathrm{GeV} / \mathrm{c})^{2}$ and $\theta_{\mathrm{CM}}=6^{\circ}$. Inner error bars represent statisitical uncertainties only, and outer error bars represent total uncertainties (statistical and systematic uncertainties)

$103 \Sigma^{0}$ channel measured cross sections vs. average kaon momentum for unbinned data. $W=2.2 \mathrm{GeV}, Q^{2} \sim 0.07(\mathrm{GeV} / \mathrm{c})^{2}$ and $\boldsymbol{\theta}_{\mathrm{CM}}=6^{\circ}$. Inner error bars represent statisitical uncertainties only, and Outer error bars represent total uncertainties (statistical and systematic uncertainties)

104 Cross sections vs. $Q^{2}$ in E94-107. Error bars represent total uncertainties (statistical and systematic uncertainties). Average variable values are listed in Table 57. 178

105 Cross sections vs. $W$ in E94-107. Error bars represent total uncertainties (statistical and systematic uncertainties). Average variable values are listed in Table 58...... 178

106 Cross section vs. $\theta_{\mathrm{CM}}$ in E94-107. Error bars represent total uncertainties (statistical and systematic uncertainties). Average variable values are listed in Table 59.

107 E94-107 electroproduction result with $Q^{2} \sim 0.07(\mathrm{GeV} / \mathrm{c})^{2}, W=2.2 \mathrm{GeV}$ and $\theta_{\mathrm{CM}}=6^{\circ}$ compared to photoproduction data and models, all of them with $W=2.2 \mathrm{GeV}$. Error bars in E94-107 result represent total uncertainty (statistical and systematic uncertainties). CLAS data from Bradford et al., [27].

108 Longitudinal to transversal component ratios of electroproduction cross section for JLab experiments. Data shown are at different $\theta_{\mathrm{CM}}$ and $W$ (on the range $1.5-1.8$ $\mathrm{GeV})$.

109 Estimate of the transverse component of the electroproduction cross section in E94$107\left(Q^{2} \sim 0.07(\mathrm{GeV} / \mathrm{c})^{2}, W=2.2 \mathrm{GeV}\right.$ and $\left.\theta_{\mathrm{CM}}=6^{\circ}\right)$ compared to photoproduction data and models, all of them with $W=2.2 \mathrm{GeV}$. Inner error bars in E94-107 estimate represent E94-107 uncertainty only. Outer error bars represent total uncertainty (E94107 uncertainty and assigned uncertainty from Figure 108). CLAS data from Bradford et al., [27].

$110 \frac{\Sigma^{0}}{\Lambda}$ cross section ratio results from E94-107. Values listed in Table 60. Average variable values listed in Tables 54 and 57.

$111 \frac{\Sigma^{0}}{\Lambda}$ photoproduction cross-section ratio data from Hall B, Jlab (Bradford et al., [27]) and E94-107 contribution at $\theta_{C M}=6^{0}$, with $Q^{2}=0.07(\mathrm{GeV} / \mathrm{c})^{2}$ and $W=2.2 \mathrm{GeV} .188$ 
112 Kaon Maid model for $\frac{\Sigma^{0}}{\Lambda}$ cross-section ratio vs. $Q^{2}$, with $W=2.2 \mathrm{GeV}$ and $\theta_{C M}=0^{0}$.

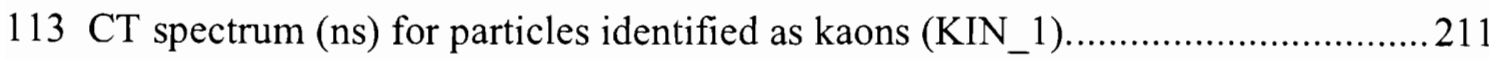

114 CT spectrum (ns) for particles identified as kaons (KIN_2) ................................211

115 CT spectrum (ns) for particles identified as kaons (KIN_3) ..............................212

116 CT spectrum (ns) for particles identified as pions (KIN_1) ..............................22

117 CT spectrum (ns) for particles identified as pions (KIN_2)...............................213

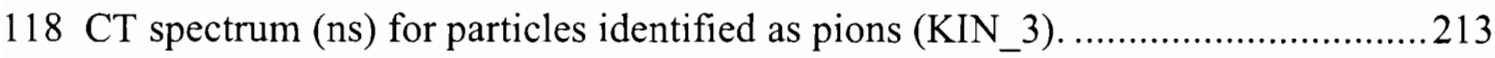

119 CT spectrum (ns) for particles identified as protons (KIN_1) ...........................2214

$120 \mathrm{CT}$ spectrum (ns) for particles identified as protons (KIN_2).............................214

121 CT spectrum (ns) for particles identified as protons (KIN_3) .............................2. 215

122 Missing mass spectrum with RICH PID cuts for KIN_1

123 Missing mass spectrum with RICH PID cuts for KIN_2

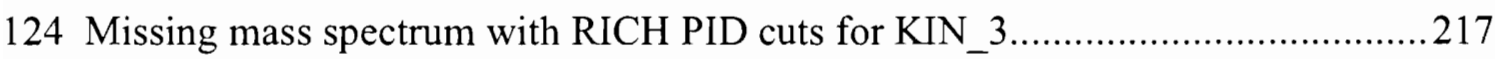

125 Background substracted spectrum with RICH PID cuts for KIN_1.....................218

126 Background substracted spectrum with RICH PID cuts for KIN_2 2......................218

127 Background substracted spectrum with RICH PID cuts for KIN_3

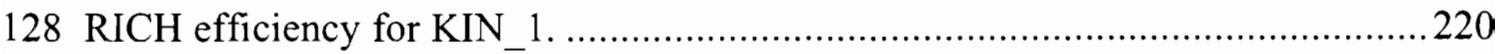

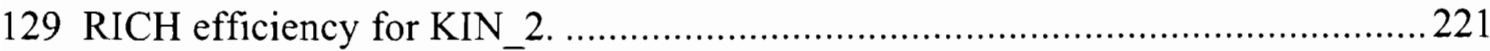

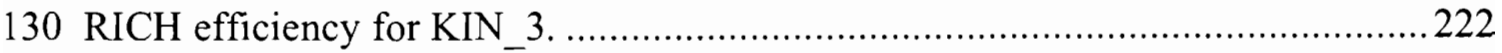


The Thomas Jefferson National Accelerator Facility (TJNAF) has unique experimental facilities. It is able to produce a high intensity electron beam up to $200 \mu \mathrm{A}$ with beam energies up to 6 gigaelectronvolts $(\mathrm{GeV})$. The high-resolution spectrometers in Hall A, among the different pieces of equipment, allow the study of sub-nuclear dynamics in the intermediate-energy range (i.e., energies of several $\mathrm{GeV}$ ). It is at these energies that a hadronic description and a quark description of nuclear reactions compete in terms of describing observations.

Quantum Hadrodynamics (QHD) constitutes a phenomenological description that uses degrees of freedom like mesons and baryons (e.g., pions, kaons, protons, neutrons, Deltas, Lambdas, Sigmas, etcetera) to describe how particles interact. Properties and strengths of interactions of those particles are determined empirically through measurements of photoproduction, electroproduction, hadron-hadron scattering, or radiative capture [1] and utilizing the symmetry relations (e.g., crossing symmetry and duality) between related reactions and the different experiments.

On the other hand, Quantum Chromodynamics (QCD) constitutes a quark description of nuclear reactions. QCD considers hadrons to be composed of a small number of more fundamental particles. These more fundamental particles are 6 quarks and 8 gluons. Therefore, QCD uses fewer particles to describe nuclear phenomena and, in principle, QHD parameters could be deduced from QCD [2]. However, calculations in QCD theory are quite difficult at few $\mathrm{GeV}$ energies, in particular due to the fact that for a given quark-gluon interaction the strength of the QCD coupling grows greater than unity 
as the transferred momentum is decreased, which constitutes an obstacle for carrying out calculations in QCD. Solutions of problems via QCD are limited to approximate methods like perturbation theory (valid for momentum transfers above some non-determined but large threshold) [2].

\subsection{Mesons, baryons and hyperons}

Hadrons are made of fundamental particles called quarks and/or antiquarks that interact through the strong force according to the standard model. Hadrons are classified into two different groups: baryons and mesons. Baryons are composed of three quarks $(q q q)$ or three anti-quarks $(\bar{q} \bar{q} \bar{q})$ and mesons are composed of quark-antiquark pairs $(q \bar{q})$. Most of the mass constituting matter and basically everything on Earth is made of up $(u)$ and down $(d)$ quarks, with no strange $(s)$ or other quarks (although virtual strange and other quarks are calculated to briefly coalesce from the vacuum before vanishing again). The lightest meson that contains strangeness is the kaon $\left(K^{+}\right)$, which is composed of an up quark and a strange antiquark $(u \bar{s})$. When baryons contain strange quarks they are called hyperons. Examples of hyperons are $\Lambda, \Sigma^{0}(u d s)$.

In experiment E94-107, Lambda $(\Lambda)$ or Sigma $\left(\Sigma^{0}\right)$ hyperons were produced together with a kaon $K^{+}$. The $\Lambda$ and $\Sigma^{0}$ particles have the same quark content. They are composed of up, down and strange quarks $(u d s)$. However, the $\Lambda$ and $\Sigma^{0}$ particles differ in their masses and also in their isospin. The lighter $\Lambda$ hyperon is an isoscalar $(I=0)$ singlet, whereas the heavier $\Sigma^{0}$ is an isovector $(I=I)$ triplet member [3]. 
Table 1 shows a selection of some of the lightest mesons and baryons composed of up, down and strange quarks and antiquarks as well as some of their main properties.

\begin{tabular}{|c|c|c|c|c|c|c|}
\hline Particle & Quarks & Mass $(\mathrm{MeV})$ & Charge & $\begin{array}{c}\text { Isospin } \\
\left|I, I_{z}\right\rangle\end{array}$ & Spin & Parity \\
\hline up quark & $u$ & $\approx 1-5$ & $+\frac{2}{3} e$ & $\left|\frac{1}{2},+\frac{1}{2}\right\rangle$ & $\frac{1}{2}$ & + \\
\hline down quark & $d$ & $\approx 3-9$ & $-\frac{1}{3} e$ & $\left|\frac{1}{2},-\frac{1}{2}\right\rangle$ & $\frac{1}{2}$ & + \\
\hline strange quark & $s$ & $\approx 60-170$ & $-\frac{1}{3} e$ & $|0,0\rangle$ & $\frac{1}{2}$ & + \\
\hline$\pi^{-}$ & $\bar{u} d$ & 139.57 & $-1 e$ & $|1,-1\rangle$ & 0 & - \\
\hline$\pi^{+}$ & $u \bar{d}$ & 139.57 & $+1 e$ & $\begin{array}{l}|1,+1\rangle \\
\left|\underline{1}+\frac{1}{\mid}\right\rangle\end{array}$ & 0 & - \\
\hline$K^{+}$ & $u \bar{s}$ & 493.68 & $+1 e$ & $-\frac{1}{2}$, & 0 & - \\
\hline$K$ & $s \bar{u}$ & 493.68 & $-1 e$ & $\left|\frac{1}{2},-\frac{1}{2}\right\rangle$ & 0 & - \\
\hline$p$ & uud & 938.27 & $+1 e$ & $\left|\frac{1}{2},+\frac{1}{2}\right\rangle$ & $\frac{1}{2}$ & + \\
\hline$n$ & $u d d$ & 939.57 & 0 & $\left|\frac{1}{2},-\frac{1}{2}\right\rangle$ & $\frac{1}{2}$ & + \\
\hline$\Lambda$ & $u d s$ & 1115.68 & 0 & $|0,0\rangle$ & $\frac{1}{2}$ & + \\
\hline$\Sigma^{0}$ & $u d s$ & 1192.64 & 0 & $|1,0\rangle$ & $\frac{1}{2}$ & + \\
\hline
\end{tabular}

Table 1: Properties of the lightest mesons and baryons [4]. 
Another interesting piece of information relevant to experiment E94-107 is how these hyperons decay. The $\Lambda$ particle decays via the weak decay either into a proton and a negative pion or into a neutron and a neutral pion. Strangeness in either decay is not conserved. The characteristic long lifetime of a weak decay is observed to be on the order of $10^{-10}$ seconds. The neutral $\Sigma^{0}$ particle decays via the strong interaction into a $\Lambda$ particle and a $\gamma$-ray conserving in this case the strangeness number with consequently a much shorter lifetime on the order of $10^{-20}$ seconds [5]. Table 2 shows the main properties of the hyperons involved in experiment E94-107:

\begin{tabular}{cccc}
\hline Hyperons & Masses $(\mathrm{MeV})$ & Lifetime $(\mathrm{s})$ & Principal decays \\
\hline$\Lambda$ & 1115.6 & $2.63 \times 10^{-10}$ & $p \pi^{-}, n \pi^{0}$ \\
$\Sigma^{0}$ & 1192.5 & $6 \times 10^{-20}$ & $\Lambda \gamma$ \\
\hline
\end{tabular}

Table 2: Main properties of the hyperons involved in experiment E94-107 [6].

\subsection{Hypernuclear physics and the elementary reaction}

Hyperons are particles that contain strangeness in their structure and are not common on Earth. On Earth, the mass comprising the majority of matter is made of "ordinary" nuclear material that contains protons and neutrons, referred to as nucleons. These nucleons sit inside the nuclei with separate nuclear orbital angular momenta, energy levels and quantum numbers (collectively describing their quantum states) and the Pauli exclusion principle prevents any two of these particles from being in the same 
quantum state. Creating a bound hyperon in the nucleus, called a hypernucleus, constitutes a different kind of matter and since a hyperon differs in structure from nucleons, it follows a separate exclusion principle. Therefore, a hyperon can have the orbital angular quantum numbers of already populated levels inside the hypernucleus. Spectroscopy experiments allow us to extract information about these energy levels where the hyperon can sit and from this we can study the way hyperons and nucleons interact inside the hypernuclei [7].

In the process of understanding hypernuclear spectroscopy experiments, it is essential to experimentally study the elementary process that creates these hyperons. One method is by studying electroproduction of hyperons, $\Lambda$ and $\Sigma^{0}$ particles, on a hydrogen target which contains only one proton (the simplest nucleus). The ratio of the two cross sections (hypernuclear and hydrogen) is proportional to the overlap of the hypernuclear wave function with the wave function of the target, which provides crucial information on the hypernuclear system. However, this elementary process is itself not very well understood so the experimental results are of great importance for theoreticians to constrain their different models. Theoretical efforts are hard in this area of research because of the additional strangeness feature in hypernuclei that increases the degrees of freedom and also greatly increases the difficulties in performing analytical calculations.

The use of an electron beam as a probe is particularly desirable because electron interactions are well understood via the theory of Quantum Electrodynamics (QED), avoiding many of the complications that arise from the influence of the strong interactions as happens in experiments utilizing hadron-hadron scattering [8,9]. The elementary electroproduction reaction is given by: 


$$
e+p \rightarrow e^{\prime}+K^{+}+\left(\Lambda \text { or } \Sigma^{0}\right)
$$

In this case an incident electron scatters off a proton by radiating a virtual photon (electromagnetic probe). The scattered electron $\left(e^{\prime}\right)$ and the ejected kaon $\left(K^{+}\right)$are then detected in coincidence. A convenient kinematics is then chosen to ensure the creation either of a Lambda $(\Lambda)$ or Sigma $\left(\Sigma^{0}\right)$ particles represented by $Y$ in Figure 1:

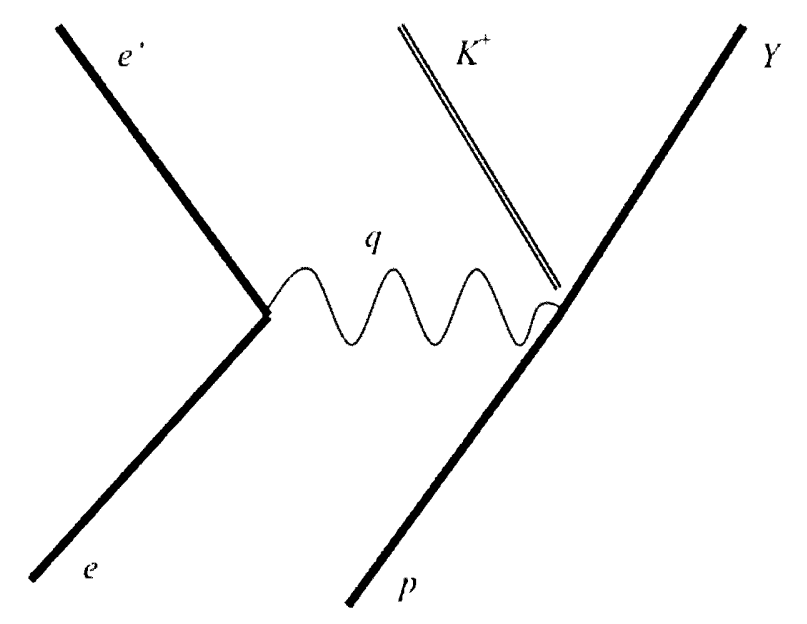

Figure 1: General view of the kinematics of the elementary reaction. 
The aim of this dissertation is the study of $\Lambda$ and $\Sigma^{0}$ electroproduction from a ${ }^{1} \mathrm{H}$ target at very forward kaon scattering angles $\left(\theta_{K}\right)$ and low 4-momentum transfer squared $\left(Q^{2}\right)$, which is an important region for understanding hyperon electroproduction via ${ }^{1} \mathrm{H}\left(e, e^{\prime} K^{+}\right) \Lambda$ and ${ }^{1} \mathrm{H}\left(e, e^{\prime} K^{+}\right) \Sigma^{0}$. My study is also an important part of the more general hypernuclear program in Hall A. Figure 2 shows the main kinematic variables for the elementary reaction:

$$
e+p \rightarrow e^{\prime}+K^{+}+Y\left(\Lambda \text { or } \Sigma^{0}\right)
$$

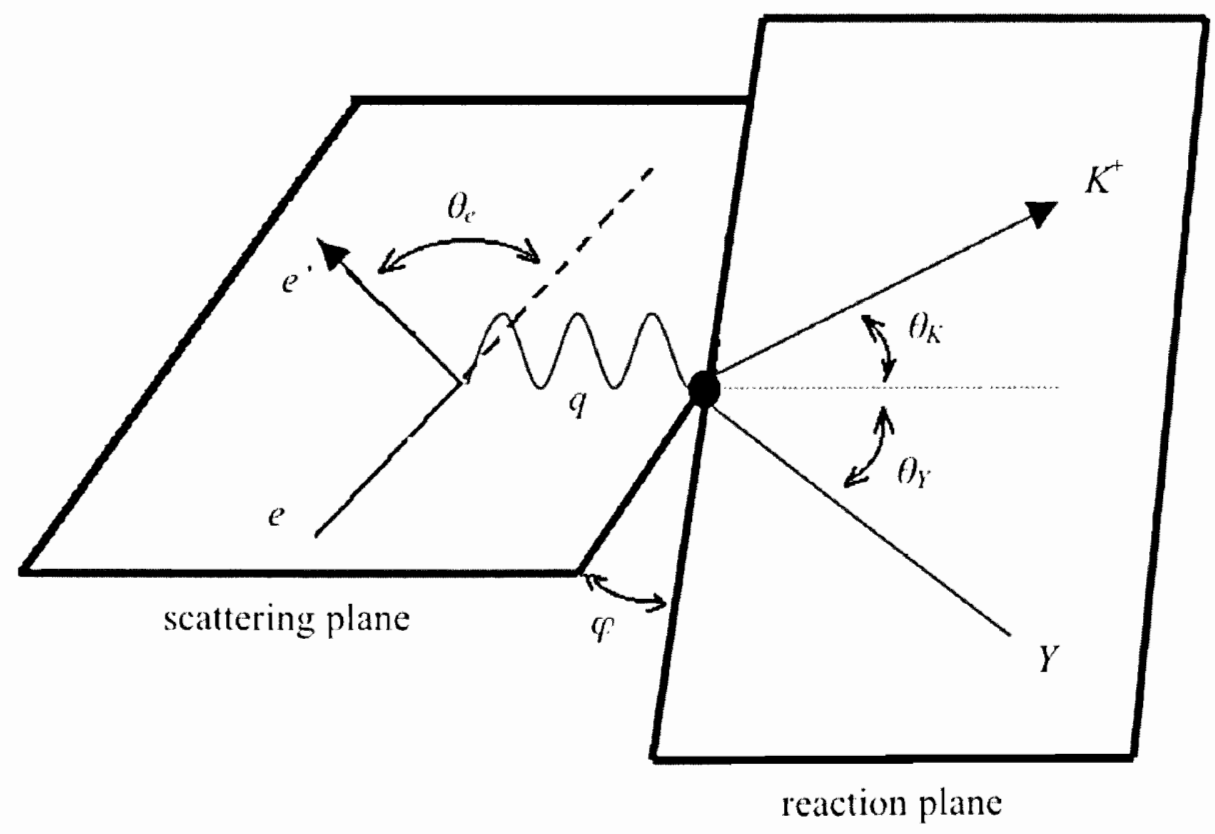

Figure 2: View of the elementary reaction kinematics. 
where the four momenta involved are:

$e=\left(E, \vec{p}_{e}\right)$, for the incident electron,

$e^{\prime}=\left(E^{\prime}, \vec{p}_{e}^{\prime}\right)$, for the scattered electron,

$p=\left(M_{p}, \overrightarrow{0}\right)$, for the target nucleon,

$k=\left(E_{K}, \vec{p}_{K}\right)$, for the produced kaon,

$Y=\left(E_{Y}, \vec{p}_{Y}\right)$, for the unobserved residual system.

Other kinematics variables including a few Lorentz invariants are defined as:

$q=(v, \vec{q})$, is the four-momentum transfer of the virtual photon,

$v=E-E^{\prime}$, is the energy of the virtual photon,

$\vec{q}=\left(\vec{p}_{e}-\vec{p}_{e}^{\prime}\right)$, is the three-vector-momentum of the virtual photon,

$q^{2}=\left(e-e^{\prime}\right)^{2}=-4 E E^{\prime} \sin ^{2}\left(\frac{\theta_{e}}{2}\right)=-Q^{2}$,

is the square of the four-momentum transfer carried by the virtual photon,

$W^{2}=(q+p)^{2}=M_{p}^{2}+2 M_{p} v-Q^{2}$,

is the mass squared of the system recoiling against the electron (i.e. the photonproton system),

$t=(q-k)^{2}=q^{2}+m_{K}^{2}-2 q k$,

is a Mandelstam variable for the four-momentum transfer squared,

$s=(e+p)^{2}=M_{p}{ }^{2}+2 M_{p} E$, 
is a Mandelstam variable for the square of the energy,

$$
u=(k-p)^{2}
$$

is a Mandelstam variable for the crossed four-momentum squared,

$$
x=\frac{Q^{2}}{2 M_{p} v}
$$

is the Bjorken scaling variable (interpreted in the quark parton model, as the fraction of the target nucleon's momentum carried by the struck quark).

The laboratory five-folded differential electroproduction cross section can be conveniently expressed in terms of the center-of-momentum $(\mathrm{CM})$ cross section. Therefore, it allows comparisons with previous or similar measurements taken at different kinematics $[10,11,12]$ :

$$
\frac{d^{5} \sigma}{d E^{\prime} d \Omega^{\prime}{ }_{e} d \Omega_{K}}=\Gamma \frac{d \cos \theta_{K}{ }^{*}}{d \cos \theta_{K}} \frac{d^{2} \sigma}{d \Omega^{*}{ }_{K}},
$$

where $\Gamma$ is the virtual-photon flux factor given by:

$$
\Gamma=\frac{\alpha}{2 \pi^{2}} \frac{\left(W^{2}-M_{p}{ }^{2}\right)}{2 M_{p}{ }^{2}} \frac{E^{\prime}}{E} \frac{1}{Q^{2}} \frac{1}{(1-\varepsilon)},
$$


and $\frac{d \cos \theta_{K}{ }^{*}}{d \cos \theta_{K}}$ is the Jacobian between the $\operatorname{CM}\left(\theta_{K}{ }^{*}\right)$ and the laboratory $\left(\theta_{K}\right)$ angle between the virtual photon and the kaon. $\varphi$ is the angle between the scattering and reaction planes as shown in Figure 2. $d \Omega_{e}^{\prime}=d \cos \theta_{e} d \varphi$ is the electron lab-frame solid angle, $d \Omega_{K}=d \cos \theta_{K} d \varphi$ is the kaon lab-frame solid angle and $d \Omega^{*}{ }_{K}=d \cos \theta^{*}{ }_{K} d \varphi$ is the kaon center-of-momentum (CM) solid angle. $\frac{d^{2} \sigma}{d \Omega^{*}{ }_{K}}$ represents the $\mathrm{CM}$ cross section and $\varepsilon$ is the virtual photon longitudinal polarization:

$$
\varepsilon=\frac{1}{1+2\left(1+\frac{v^{2}}{Q^{2}}\right) \tan ^{2}\left(\frac{\theta_{e}}{2}\right)}
$$

the cross section for kaon electroproduction in the $\mathrm{CM}$ frame can be written as follows $[12,13,14]:$

$$
\frac{d^{2} \sigma}{d \Omega^{*}{ }_{K}}=\left(\frac{d^{2} \sigma_{T}}{d \Omega^{*}{ }_{K}}\right)+\varepsilon\left(\frac{d^{2} \sigma_{L}}{d \Omega^{*}{ }_{K}}\right)+\varepsilon\left(\frac{d^{2} \sigma_{T T}}{d \Omega^{*}{ }_{K}}\right) \cos (2 \varphi)+\sqrt{2 \varepsilon(\varepsilon+1)}\left(\frac{d^{2} \sigma_{L T}}{d \Omega^{*}{ }_{K}}\right) \cos (\varphi),
$$

or in more compact notation:

$$
\frac{d^{2} \sigma}{d \Omega^{*}{ }_{K}}=\sigma_{T}+\varepsilon \sigma_{L}+\varepsilon \cos (2 \varphi) \sigma_{T T}+\sqrt{2 \varepsilon(\varepsilon+1)} \cos (\varphi) \sigma_{L T}
$$


and where:

$\sigma_{T}$ is the cross section that results from the transverse component of the virtual photons,

$\sigma_{L}$ is the cross section that results from the longitudinal component of the virtual photons,

$\sigma_{L T}$ is the cross section that results from the interference between the transverse and longitudinal components of the virtual photons, $\sigma_{T T}$ is the cross section that results from the interference between the two different states of the transverse components of the virtual photons.

The quantities $\sigma_{T}, \sigma_{L}, \sigma_{T T}$ and $\sigma_{L T}$ completely characterize the dependence of the cross section for scattering electrons off of the nucleon (nucleus). They are functions of the independent kinematical variables $Q^{2}, W$ and $t$.

It is interesting to point out that if Equation (19) is integrated in $\varphi$ over $(0,2 \pi)$ the interference terms vanish. In practical terms this means that if the experiment is carried out in such way that the acceptance has complete coverage in $\varphi$ then the interference terms vanish. Further, if data could then be taken at different $\varepsilon$ holding the other variables constant, a Rosenbluth technique can be used to separate $\sigma_{L}$ and $\sigma_{T}[13]$. However, this is not the case in E94-107, as will be shown in Chapter 4, because in this experiment the angular acceptance is not uniform in $\varphi$. Consequently, the results from E94-107 include all four of these response functions. 
Various theoretical models have been created in an attempt to describe kaon production and radiative kaon capture while maintaining consistency with SU(3) symmetry constraints on the coupling constants [15]. Most of these models have been constructed for energies of just a few GeV. These energies are low enough that the models can be formulated under the effective rules of Quantum Hadrodynamics (QHD). Moreover, when these models are tested at slightly higher energies they are consistent with perturbative Quantum Chromodynamics (pQCD) [1]. The most important models in this energy regime are isobaric models and Regge models.

\subsubsection{Isobaric models}

In isobaric models, the kaon production amplitudes are calculated from tree-level QHD Feynman diagrams. In other words, the models include only one-particle exchange. The tree level Feynman diagrams are for $s, t$ and $u$ channels. Figure 3 shows the three channels of processes. Basically, in the $s$ channel one considers the virtual photon $(q)$ as being absorbed by the proton $(p)$ which then decays emitting a meson $\left(K^{+}\right)$and a hyperon $(Y)$. The proton could also go through an intermediate resonance state before the emission as shown in Figure 3. The $t$ channel represents the virtual photon being scattered by a (virtual) $K^{+}$meson or one of its resonances so that a $K^{+}$and a hyperon are emitted. The $u$ channel treats the scattering as the virtual photon being absorbed by a hyperon (or hyperon resonance), which then emits a $K^{+}$and another hyperon. 
Different models include different couplings between the particles and different resonances. When these different model calculations are performed limits are taken on the properties of the propagators. The strengths of the couplings are empirical degrees of freedom determined by fitting the experimental data. A model described in Reference [16] uses a sum over $s$-channel nucleonic resonances up to spin $5 / 2, u$-channel hyperonic resonances (spin 1/2) and the $t$-channel kaonic resonances $K^{*}(892)$ and $K 1(1270)$. In another model, called the WJC model [15], a different selection of $s$ and $u$ channels resonances is used. In these models, each vertex in the diagrams shown in Figure 3 is associated with a particular coupling constant.
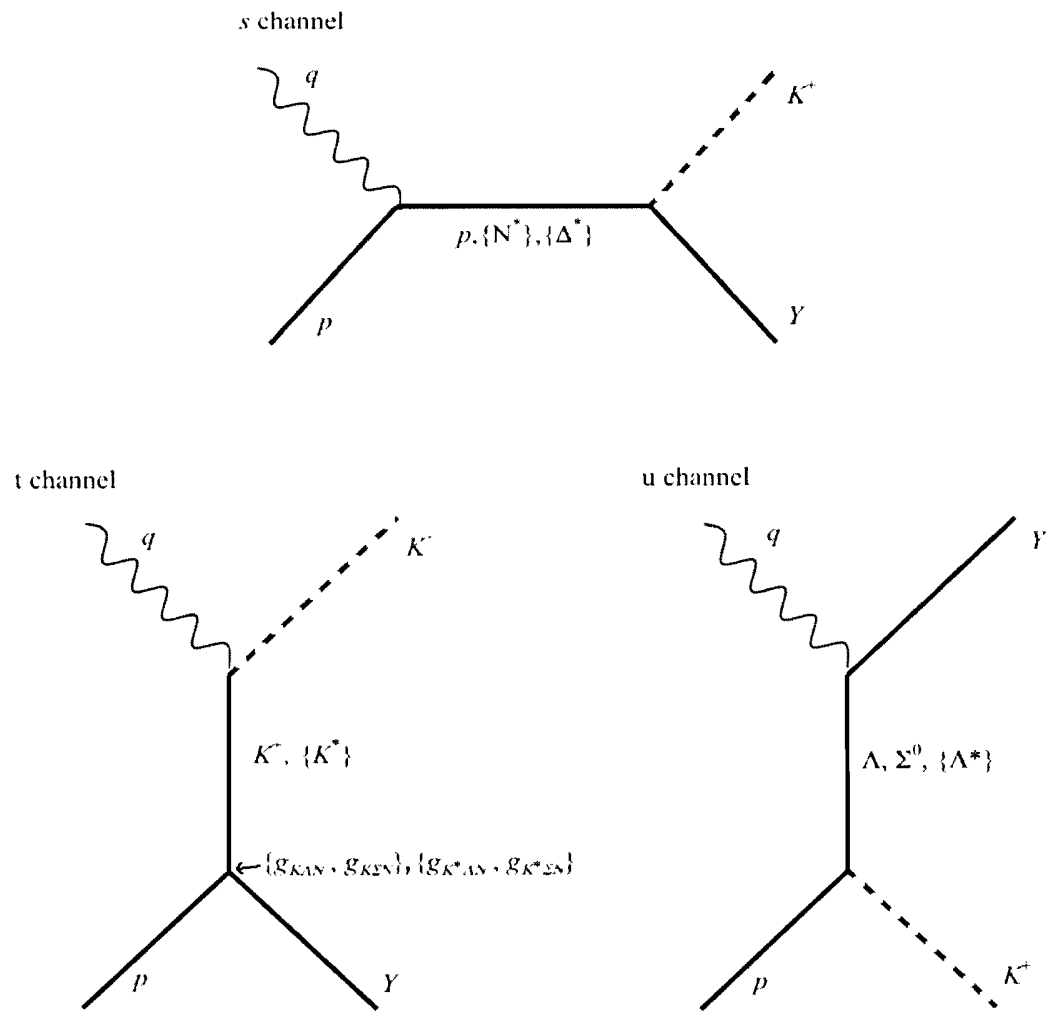

Figure 3: Feynman diagramas in isobaric models for kaon electroproduction. 
All the isobaric models start by including only spin $1 / 2$ baryonic resonances. These resonances can be different for each model and the coupling constants are obtained by performing phenomenological fits to the data. There are not enough data available to fully constrain the coupling constants. New data (especially at forward kaon angles where there are very few data) are important to discriminate between which of the isobaric models is best able to describe the experimental observations. J.C. David, G. Fayard, G.H. Lamot and B. Saghai [16] compared four different isobaric models called AS [17], WJC [15], SL [16] and MBH [18]. To perform the fits they started with the available data with energy range from threshold to $2.1 \mathrm{GeV}$, and by extending the AS model to higher energies and focusing on the $K^{+} \Lambda$ photoproduction channel they included the spin$3 / 2$ nucleonic resonances. The $K^{+} \Lambda$ electroproduction channel was also studied and in this case spin-5/2 nucleonic resonances were included resulting in the Saclay-Lyon (SL) model. See Table 3 for the $K \Lambda$ channels. In the case of the $K^{+} \Sigma$ channels, the fits were done adding up to isospin-3/2 nucleonic resonances. The resulting SL model is shown. See Table 4 and Reference [16]. 


\begin{tabular}{ccccc}
\hline Particle & Coupling & AS & WJC & SL \\
\hline$\Lambda$ & $g_{K A N} / \sqrt{4 \pi}$ & $-4.17 \pm 0.75$ & -2.38 & $-3.16 \pm 0.01$ \\
$\Sigma$ & $g_{K \Sigma N} / \sqrt{4 \pi}$ & $1.18 \pm 0.66$ & 0.27 & $0.91 \pm 0.10$ \\
& & & & \\
$K^{*}$ & $G_{V} / 4 \pi$ & $-0.43 \pm 0.07$ & -0.16 & $-0.05 \pm 0.01$ \\
& $G_{T} / 4 \pi$ & $0.20 \pm 0.12$ & 0.08 & $0.16 \pm 0.02$ \\
$K 1$ & $G_{V I} / 4 \pi$ & $-0.10 \pm 0.06$ & 0.02 & $-0.19 \pm 0.01$ \\
& $G_{T I} / 4 \pi$ & $-1.21 \pm 0.33$ & 0.17 & $-0.35 \pm 0.03$ \\
& & & & \\
$N 1$ & $G_{N 1} / \sqrt{4 \pi}$ & $-1.41 \pm 0.60$ & & $-0.01 \pm 0.12$ \\
$N 4$ & $G_{N 4} / \sqrt{4 \pi}$ & & -0.04 & \\
$N 6$ & $G_{N 6} / \sqrt{4 \pi}$ & & -0.06 & \\
$N 7$ & $G^{a}{ }_{N 7} / 4 \pi$ & & & $-0.04 \pm 0.01$ \\
& $G^{b}{ }_{N 7} / 4 \pi$ & & & $-0.14 \pm 0.04$ \\
$N 8$ & $G^{a}{ }_{N 8} / 4 \pi$ & & & $-0.63 \pm 0.10$ \\
& $G^{b}{ }_{N 7} / 4 \pi$ & & & $-0.05 \pm 0.56$ \\
& & & -0.07 & $-0.31 \pm 0.06$ \\
$L 1$ & $G_{L I} / \sqrt{4 \pi}$ & & & $-1.18 \pm 0.09$ \\
$L 3$ & $G_{L 3} / \sqrt{4 \pi}$ & $-3.17 \pm 0.86$ & & $-4.96 \pm 0.19$ \\
$L 5$ & $G_{L S} / \sqrt{4 \pi}$ & &
\end{tabular}

Table 3: Isobaric model coupling constants and resonances for $K \Lambda$ channels [16]. 


\begin{tabular}{|c|c|c|c|c|}
\hline Particle & Coupling & $\mathrm{MBH}$ & WJC & $\mathrm{SL}$ \\
\hline$\Lambda$ & $g_{K A N} \sqrt{4 \pi}$ & 0.51 & -2.38 & $-3.23 \pm 0.17$ \\
\hline$\Sigma$ & $g_{K \Sigma N} \sqrt{4 \pi}$ & 0.13 & 0.27 & $0.80 \pm 0.10$ \\
\hline \multirow[t]{2}{*}{$K^{*}$} & $G_{V} / 4 \pi$ & 0.05 & 0.11 & $0.02 \pm 0.01$ \\
\hline & $G_{T} / 4 \pi$ & 0.05 & -0.14 & $-0.07 \pm 0.02$ \\
\hline \multirow[t]{2}{*}{$K 1$} & $G_{V I} / 4 \pi$ & & -0.13 & $-0.05 \pm 0.01$ \\
\hline & $G_{T 1} / 4 \pi$ & & 0.07 & $0.23 \pm 0.04$ \\
\hline$N 1$ & $G_{N I} / \sqrt{4 \pi}$ & & & $-0.95 \pm 0.11$ \\
\hline N4 & $G_{N 4} / \sqrt{4 \pi}$ & 0.08 & 0.09 & \\
\hline N6 & $G_{N \sigma} / \sqrt{4 \pi}$ & 0.57 & 0.47 & \\
\hline \multirow[t]{2}{*}{$N 7$} & $G^{a}{ }_{N 7} / 4 \pi$ & & & $-0.04 \pm 0.02$ \\
\hline & $G^{b}{ }_{N 7} / 4 \pi$ & & & $-0.53 \pm 0.06$ \\
\hline \multirow[t]{2}{*}{ N8 } & $G^{a}{ }_{N 8} / 4 \pi$ & & & $2.02 \pm 0.20$ \\
\hline & $G^{b}{ }_{N 7} / 4 \pi$ & & & $3.91 \pm 0.57$ \\
\hline$L 1$ & $G_{L l} / \sqrt{4 \pi}$ & & 0.46 & $-0.42 \pm 0.03$ \\
\hline$L 3$ & $G_{L 3} / \sqrt{4 \pi}$ & & & $-0.10 \pm 0.09$ \\
\hline$L 5$ & $G_{L 5} / \sqrt{4 \pi}$ & & & $6.01 \pm 0.23$ \\
\hline$S 1$ & $G_{S I} / \sqrt{4 \pi}$ & & & $-1.72 \pm 0.21$ \\
\hline DI & $G_{D I} / \sqrt{4 \pi}$ & & -0.03 & \\
\hline D2 & $G_{D 2} / \sqrt{4 \pi}$ & 0.07 & -0.06 & \\
\hline D3 & $G_{D 3} / \sqrt{4 \pi}$ & 0.30 & -0.51 & $0.43 \pm 0.04$ \\
\hline \multirow[t]{2}{*}{ D4 } & $G^{a}{ }_{D 4} / 4 \pi$ & & & $-0.47 \pm 0.06$ \\
\hline & $G^{b}{ }_{D 4} / 4 \pi$ & & & $-1.88 \pm 0.14$ \\
\hline \multirow[t]{2}{*}{ D7 } & $G^{a}{ }_{D 7} / 4 \pi$ & & & $0.05 \pm 0.01$ \\
\hline & $G^{b}{ }_{D 7} / 4 \pi$ & & & $0.29 \pm 0.04$ \\
\hline
\end{tabular}

Table 4: Isobaric model coupling and resonances for $K \Sigma$ channels [16].

The different coupling constants are values are shown in Tables 3 and 4 . The tables show that the fits of the different resonances (i.e., degrees of freedom in the fits) used in the calculations result in disagreements in the coupling constants. One reason for this disagreement is the lack of sufficient data in kaon production to constrain the models. 
Additional electromagnetic kaon production data in previously unmeasured regions (i.e., forward kaon angles) are important for improving the results in the theoretical calculations by constraining the model parameters.

\subsubsection{Regge models}

A different class of models is based on Regge theory [19]. In Regge models the Feynman diagram approach is modified in such a way that at higher energies the standard single particle Feynman propagator $\frac{1}{\left(t-m^{2}\right)}$ is replaced by the Regge propagator that takes into account the exchange of a family of particles with the same intrinsic quantum numbers [20]. As an example, in photoproduction the pseudoscalar hadronic current in the $t$-channel exchange for the $\Lambda$ channel is given by:

$$
J_{K_{t-\text { exch }}}^{\mu} \alpha \operatorname{iek} \bar{Y}_{f}\left(k-p_{K}\right)^{\mu} \times \mathrm{P}_{\mathrm{Re} g g e}^{K} \times g_{K \Lambda N} \gamma^{5} N_{i} .
$$

In Equation (20) $e$ is the fundamental charge, $Y_{f}$ is the Lambda spinor, $k^{\mu}=\left(q-p_{K}\right)^{\mu}$ is the four-vector of the exchanged kaon and $k$ is its Feynman dagger, where $p_{K}^{\mu}$ is the kaon four-vector and $q^{\mu}$ is the photon four-vector, $\mathrm{P}_{\mathrm{Regge}}^{K}$ represents the Regge propagator, $g_{K \wedge N}$ is the coupling constant at the $p^{+}-\Lambda-K^{+}$vertex in the diagram and $N_{i}$ is the proton spinor.

The Regge propagator has the form: 


$$
\mathrm{P}_{\mathrm{Re} g g e}^{K}=\left(\frac{s}{s_{n}}\right)^{\alpha_{K}(t)}\left(\frac{\pi \alpha_{K}^{\prime}(t)}{\sin \pi \alpha_{K}(t)}\right)\left(\frac{S_{K}+e^{-i \pi \alpha_{K}(t)}}{2}\right)\left(\frac{1}{\Gamma\left(1+\alpha_{K}(t)\right)}\right),
$$

where $s$ is the Mandelstam variable and $s_{o}$ is a mass scale, commonly taken as $1 \mathrm{GeV}^{2}$. The $\alpha_{K}$ and $\alpha_{K}^{\prime}$ parameters characterize the Regge trajectories shown in Figure 4. $S_{K}$ with values +1 or -1 is the signature of the trajectory and $\Gamma$ is the Gamma function $\left(\Gamma(x)=\int_{0}^{\infty} t^{x-1} e^{-t} d t\right)$

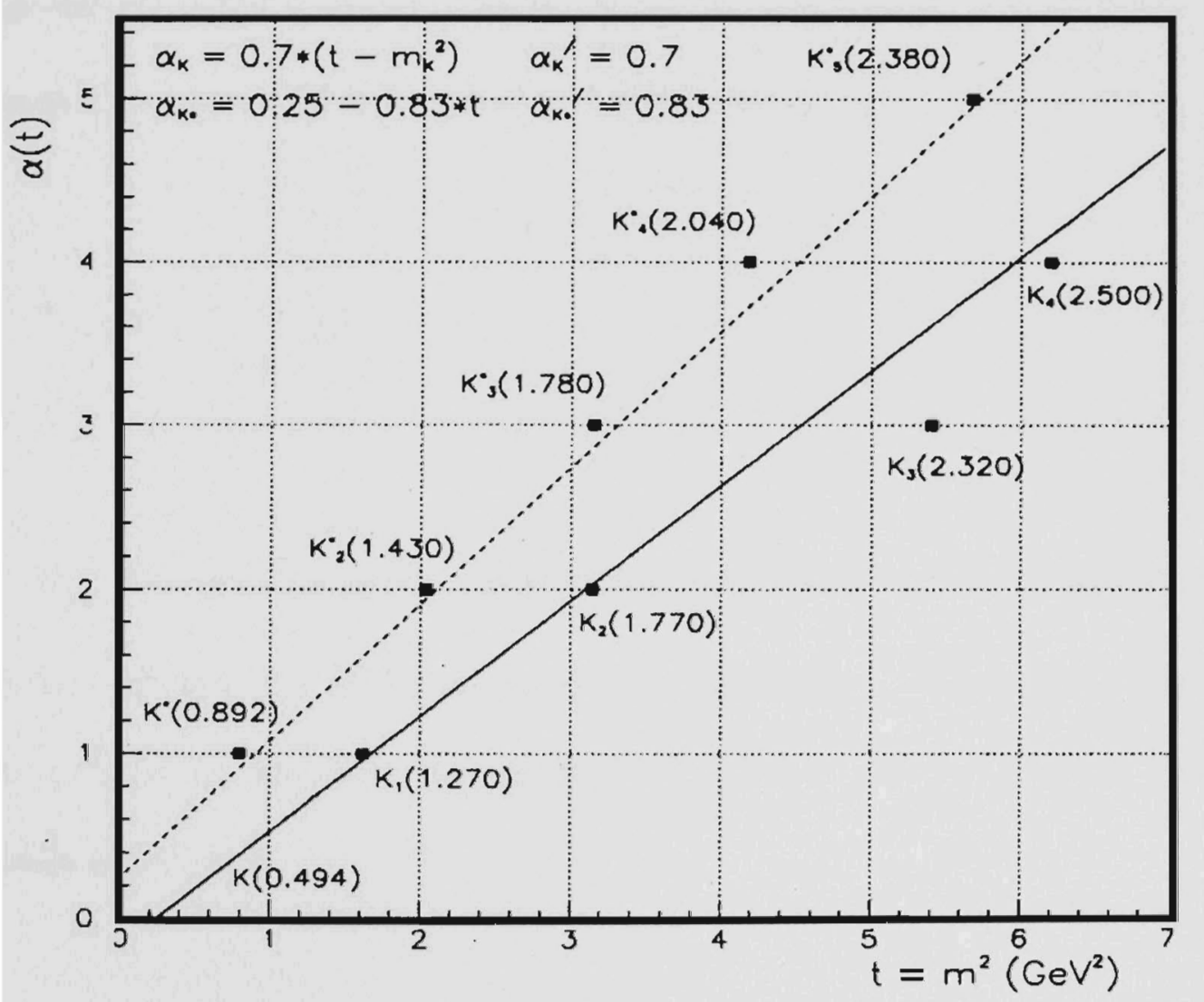

Figure 4: Regge trajectories of the form $\alpha=\alpha_{0}+\alpha^{\prime *} t$ for $K$ and $K^{*}$ [20]. 
The equation for the Regge propagator (21) reduces to the Feynman propagator when one approaches the first pole of the trajectory. In other words, this occurs when $t \rightarrow m_{K}^{2}$. A similar current operator and propagator can be constructed for the pseudovector coupling of the $K^{*}$ mesons. The only parameters in Regge model described in [19] are the first materialization of the trajectories with the external particles, that is to say $g_{K \Lambda N}, g_{K^{*} \Lambda N}, \ldots$ which have to be determined to describe all existing high-energy data $[20]$.

An extension from photoproduction to electroproduction can be done by multiplying the gauge invariant $t$-channel $K$ and $K^{*}$ diagrams by a form factor. The monopole form factor used in the model [19] is given by:

$$
F_{K, K^{*}}\left(Q^{2}\right)=\frac{1}{1+\frac{Q^{2}}{\Lambda_{K, K^{*}}^{2}}},
$$

where $\Lambda_{K, K^{*}}^{2}$ are adjustable parameters known as mass scales. The mass scales can be fit to the high $Q^{2}$ behavior of the separated electroproduction cross sections $\sigma_{T}$ and $\sigma_{L}$, which is useful in describing the results of Rosenbluth or other response function separation experiments. 


\subsection{3 $\Sigma^{0} / \Lambda$ production ratio}

From previous measurements of the ${ }^{1} \mathrm{H}\left(e, e^{\prime} K^{+}\right) \Lambda, \Sigma^{0}$ reactions it has been observed that the ratio of cross sections $\Sigma^{0} / \Lambda$ for the two channels decreases rapidly as $Q^{2}$ is increased. The following Figures 5 and 6 show world data up to 1996 from which it is clear to see that $\Sigma^{0} / \Lambda$ production ratio decreases with $Q^{2}$ [11]. The data were taken at a variety of values of $W$ and kaon angles $\left(\theta_{K}\right)$. The data have been extrapolated to a common value of $W=2.15 \mathrm{GeV}$, using the method found in References $[11,13]$. The angles $\left(\theta_{K}\right)$ were all forward, but have not been extrapolated to $0^{\circ}$.

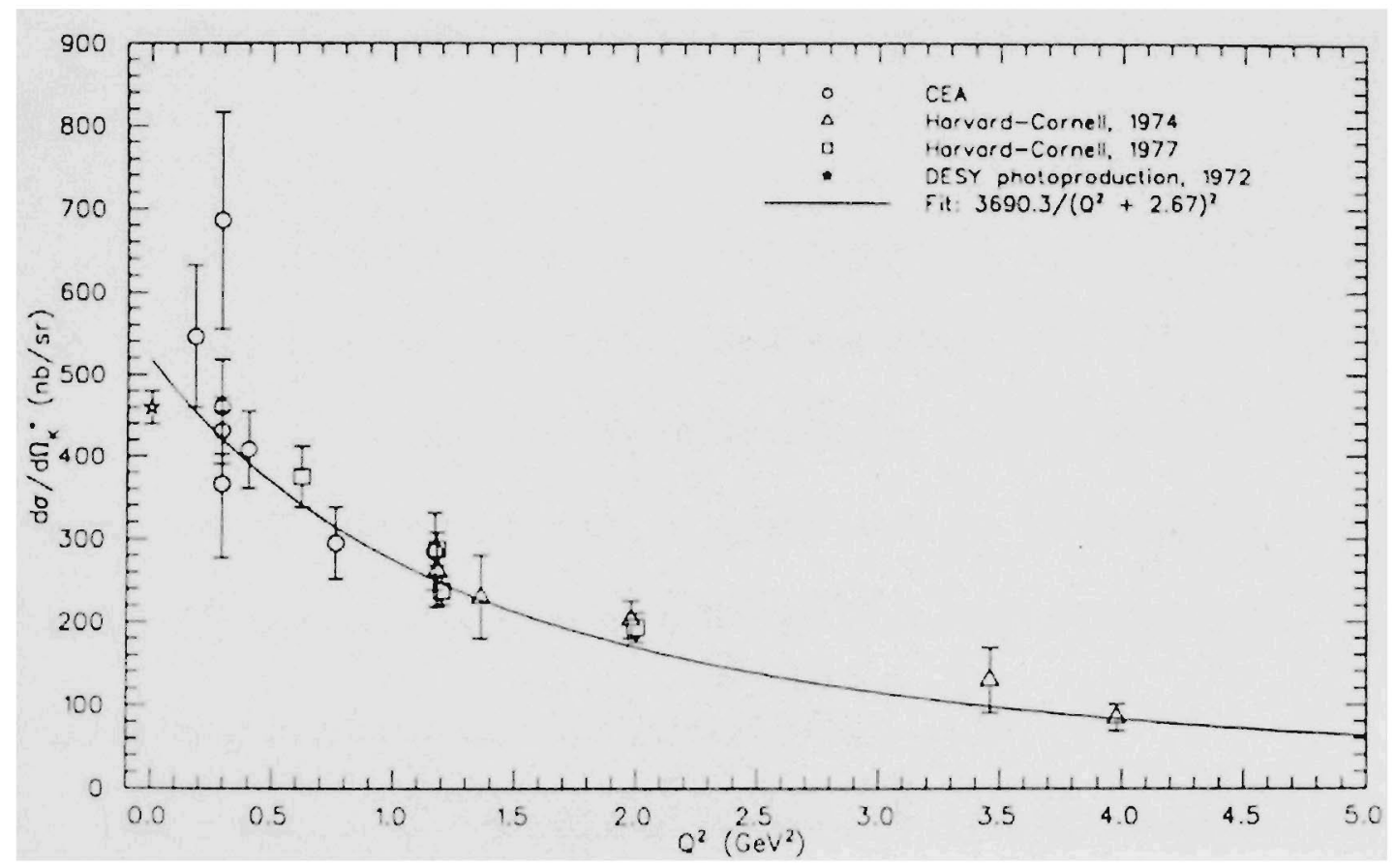

Figure 5: World data prior to 1996 for ${ }^{1} \mathrm{H}\left(e, e^{\prime} K^{+}\right) \Lambda,\langle W\rangle=2.15 \mathrm{GeV}, \theta_{C M}=0$ [11]. 


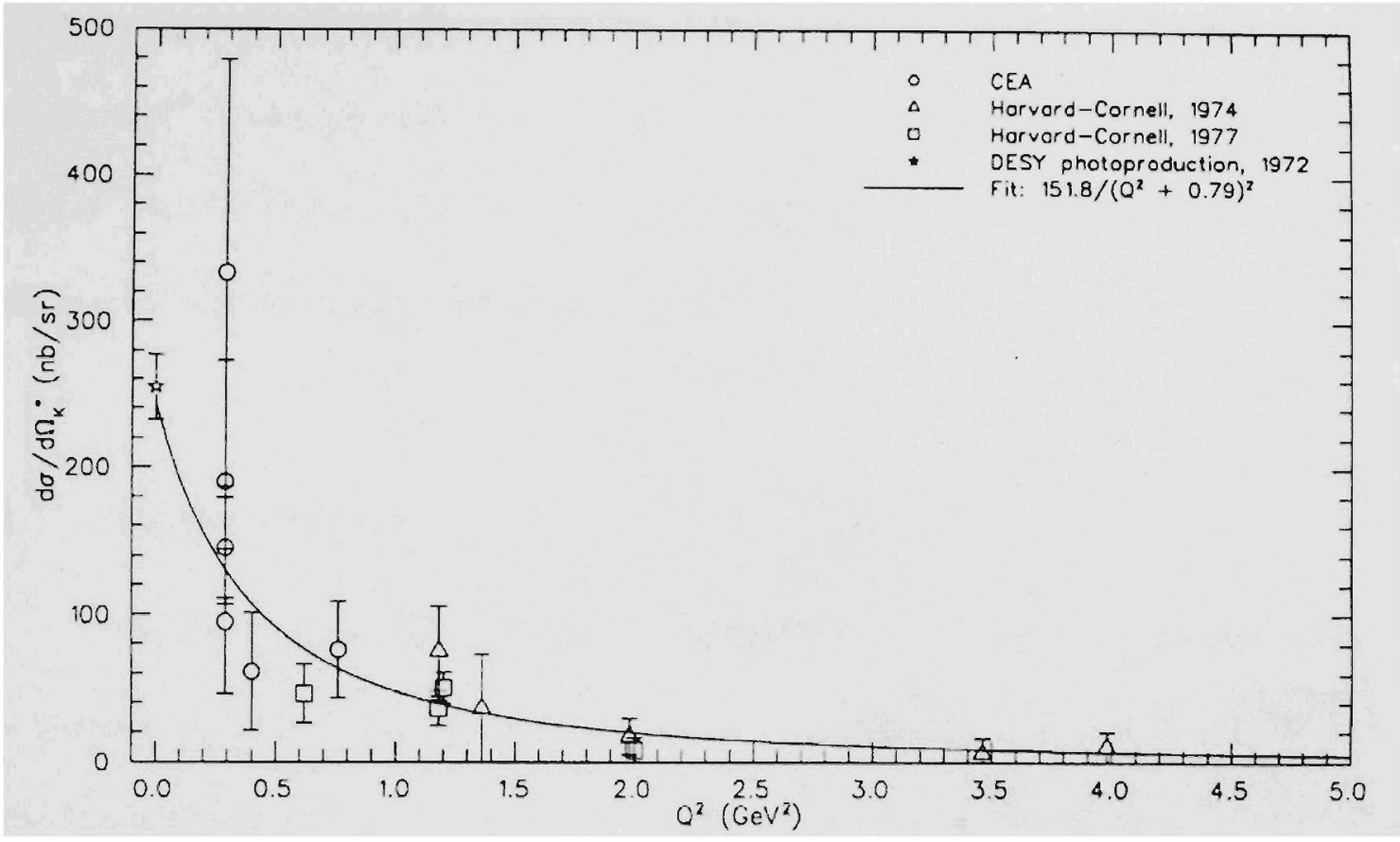

Figure 6: World data prior to 1996 for ${ }^{1} \mathrm{H}\left(e, e^{\prime} K^{+}\right) \Sigma^{0},\langle W\rangle=2.15 \mathrm{GeV}, \theta_{C M}=0$ [11].

In Figures 5 and 6 there is a photoproduction point $\left(Q^{2}=0\right)$; these figures suggest that the cross section for electroproduction of kaons at very forward angles and very low $Q^{2}$ is larger than the cross section for photoproduction [21], which rises one of the questions that this dissertation tries to answer with the measurements done on ${ }^{1} \mathrm{H}\left(e, e^{\prime} K^{+}\right) \Lambda, \Sigma^{0}$ cross sections at low $Q^{2} \sim 0.07 \mathrm{GeV}^{2}$ and a laboratory kaon angle $\theta=6^{\circ}$, corresponding to a center-of-mass (CM) kaon angle of $\theta_{C M}=6^{\circ}$.

On the basis of a simple isospin argument, the $\Sigma^{0} / \Lambda$ production ratio should decrease with $Q^{2}$. This argument is formulated in the framework of the quark parton model and is qualitative. The decrease of the $\Sigma^{0} / \Lambda$ ratio is related to the decrease of the ratio $F_{l}^{q n} / F_{1}^{q p}$ as $Q^{2}$ increases, where $F_{l}^{q p}$ and $F_{l}^{q n}$ are the usual structure functions for 
deep inelastic electron-nucleon scattering. Nachtmann and Close have shown that the observed $\Sigma^{0} / \Lambda$ ratio decreases with $Q^{2}$ using this isospin argument $[20,21,22,23,24]$. The previous references also show the $\Sigma^{0} / \Lambda$ ratio depends on the Bjorken scale $x$, which shows the expected dependence on $Q^{2}$ as well [25].

\subsection{Physics motivation}

The study of $\Lambda$ and $\Sigma^{0}$ electroproduction from a ${ }^{1} \mathrm{H}$ target at very forward kaon scattering angles and low 4-momentum transfer squared $\left(Q^{2}\right)$ is one of the objectives in the hypernuclear program at Hall A, JLab, which is an important region for measuring hyperon electroproduction via ${ }^{1} \mathrm{H}\left(e, e^{\prime} K^{+}\right) \Lambda$ and ${ }^{1} \mathrm{H}\left(e, e^{\prime} K^{+}\right) \Sigma^{0}$. At small kaon angles and low $Q^{2}$, theoretical models of the $\Lambda$ cross section vary greatly. The $\Sigma^{0}$ data also provide important information on the $\Sigma^{0} / \Lambda$ ratio at low $Q^{2}$ where this ratio changes rapidly as explained in Section 1.4 .

Currently existing models are divergent at small kaon (forward) $\mathrm{CM}$ angles and the existing data on elementary hyperon production at small forward angles is not enough to fully constrain the models. In addition most of the data have been taken at kaon CM angles larger than 20 degrees. Figure 7 shows that the deviations in the various models become large at small (forward) kaon CM angles. The models contain a number of free parameters (such as, e.g., coupling constants and cut-off masses), which can be constrained by low angle data. Since perturbative QCD is not applicable, the parameters of the models rely upon phenomenological approaches that vary from model to model. The description of the elementary process is, however, poor in the kinematical region 
relevant for the hypernuclear calculations (e.g., for photon energies larger than $1.5 \mathrm{GeV}$ and small photon mass $\left.Q^{2} \sim 0.06(\mathrm{GeV} / \mathrm{c})^{2}\right)$, which covers very forward kaon angles.

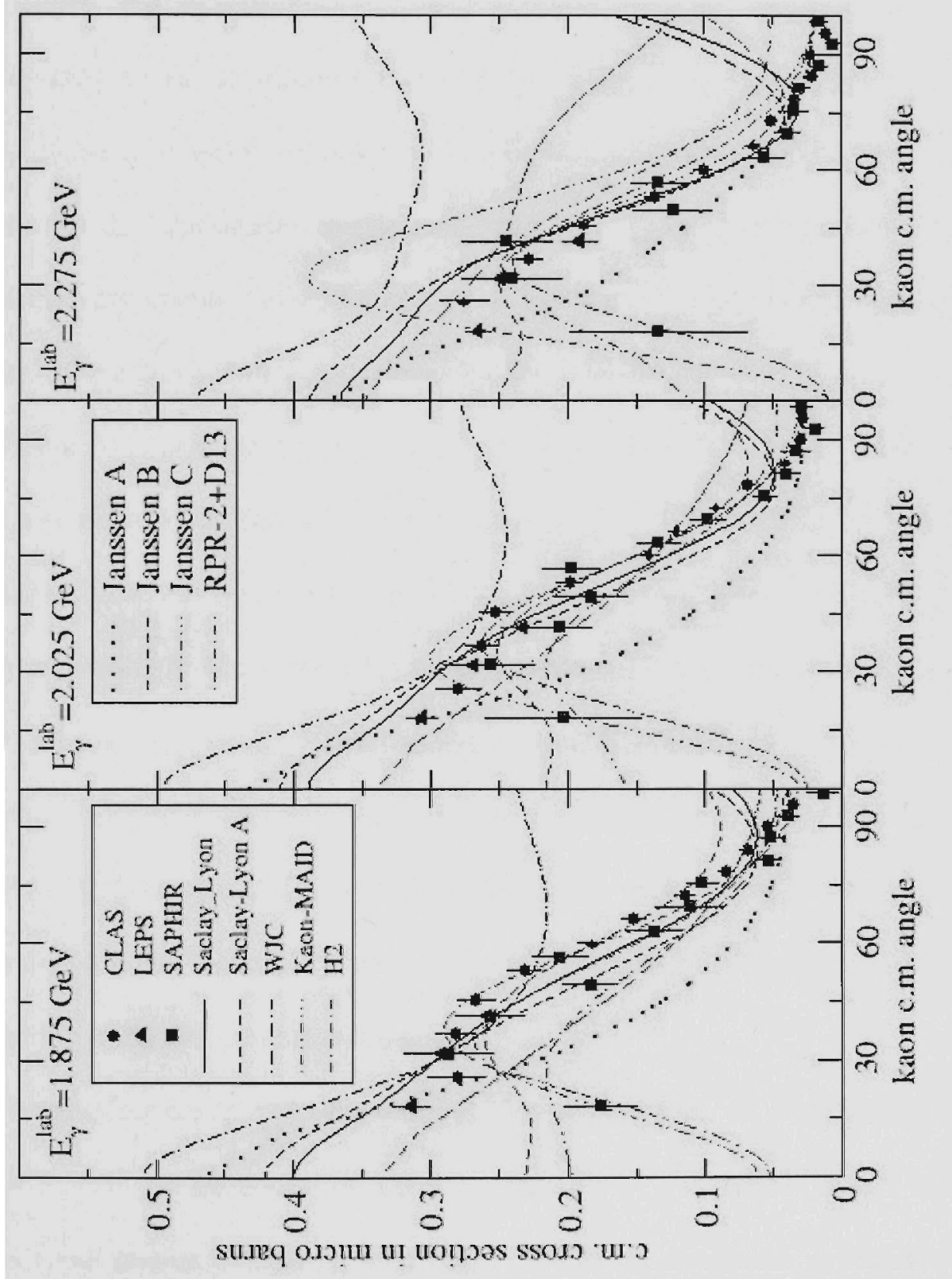

Figure 7: Isobaric model predictions compared with experimental data [26]. 
Calculations of the cross section for production of hypernuclei in excited states, e.g., to analyze data from the hypernuclear experiments carried out at Jefferson Laboratory, depend on two main ingredients: i) the elementary-production operator and ii) the nuclear and hypernuclear structure information. One has to understand the elementary process, in the relevant kinetical region to learn more about the hypernuclear structure, which is closely connected with the hyperon-nucleon interaction. However, predictions for the hypernuclear production cross sections using various isobaric models for the elementary operator differ by more than $100 \%$ at kaon laboratory angles less than 10 degrees and photon energies larger than $1.5 \mathrm{GeV}$ (See Figure 7).

Figure 7 shows predictions of the models discussed above, compared with experimental photoproduction $\left(Q^{2}=0\right)$ data from CLAS [27], SAPHIR [28], and LEPS [29] collaborations. An updated version of the hybrid RPR-2 model [30] (Regge-isobar approach) is shown in which the $\mathrm{P} 11(1900)$ resonance is replaced by the D13(1900) to better describe the latest data. Kaon-MAID, Janssen C, and H2 [31] contain a hadronic form factor (HFF), revealing very sharp damping of the cross section at kaon CM angles smaller than $30^{\circ}$, whereas the cross section in models without a HFF, Saclay-Lyon and Williams-Ji-Cotanch (WJC) [15], continue rising (Figure 7). The CLAS and SAPHIR data suggest a plateau at these forward angles.

The possible damping of the cross section at very forward angles and photon lab energy of $2 \mathrm{GeV}$ can be understood within the Saclay-Lyon A (SLA) and H2 models [31]. The cross section calculated with these models is dominated by the Born terms, particularly by the electric part of the photon exchange, at kaon $\mathrm{CM}$ angles smaller than 30 degrees. In this case, the contribution provides a plateau in the cross section as the 
angle goes to zero for the SLA results, such a feature would be very similar in both models since the main two coupling constants are almost equal. The situation is different in the $\mathrm{H} 2$ model due to a very strong suppression of the photon term by the HFF, which is of the order of $0.2 \mu \mathrm{b}$ in cross section for this energy region. The very strong suppression of the Born terms leads to a pronounced "dip" in the differential cross section at very small angles (see Figure 7 for the results of the $\mathrm{H} 2$ model). The Kaon MAID model gives a similar result. In this region, the resonances contribute only by interference with the Born terms so that they cannot fill in the dip.

The strong suppression of the cross section at very forward angles and energies above $1.7 \mathrm{GeV}$ is a common feature of the isobaric models with a HFF. Therefore, the dynamical aspect of the isobaric models requires a more detailed investigation. However, the large bulk of new data cannot discriminate between the models due to a lack of data at very forward angles. These latest data were measured only for CM kaon angles as small as 18 and 26 degrees for the LEPS, SAPHIR and CLAS sets respectively, see Figure 7.

Analysis of CLAS data on the cross section in a wide energy interval [27] shows, besides the forward peaking, a progressive flattening of the slope as the kaon angle goes to zero. The CLAS data, however, only reach to 26 degrees. The question whether the cross section continues rising, forms a plateau or decreases (as is the case for the highenergy data over $5 \mathrm{GeV}[20]$ ), as some of the models predict, is therefore another challenging question.

On top of that, there is a need for a better description of the elementary operator at forward angles, to serve as input in the hypernuclear calculations. The scarceness of data 
at forward angles can result only in a poor description of the process and therefore in a large uncertainty in the hypernuclear calculations.

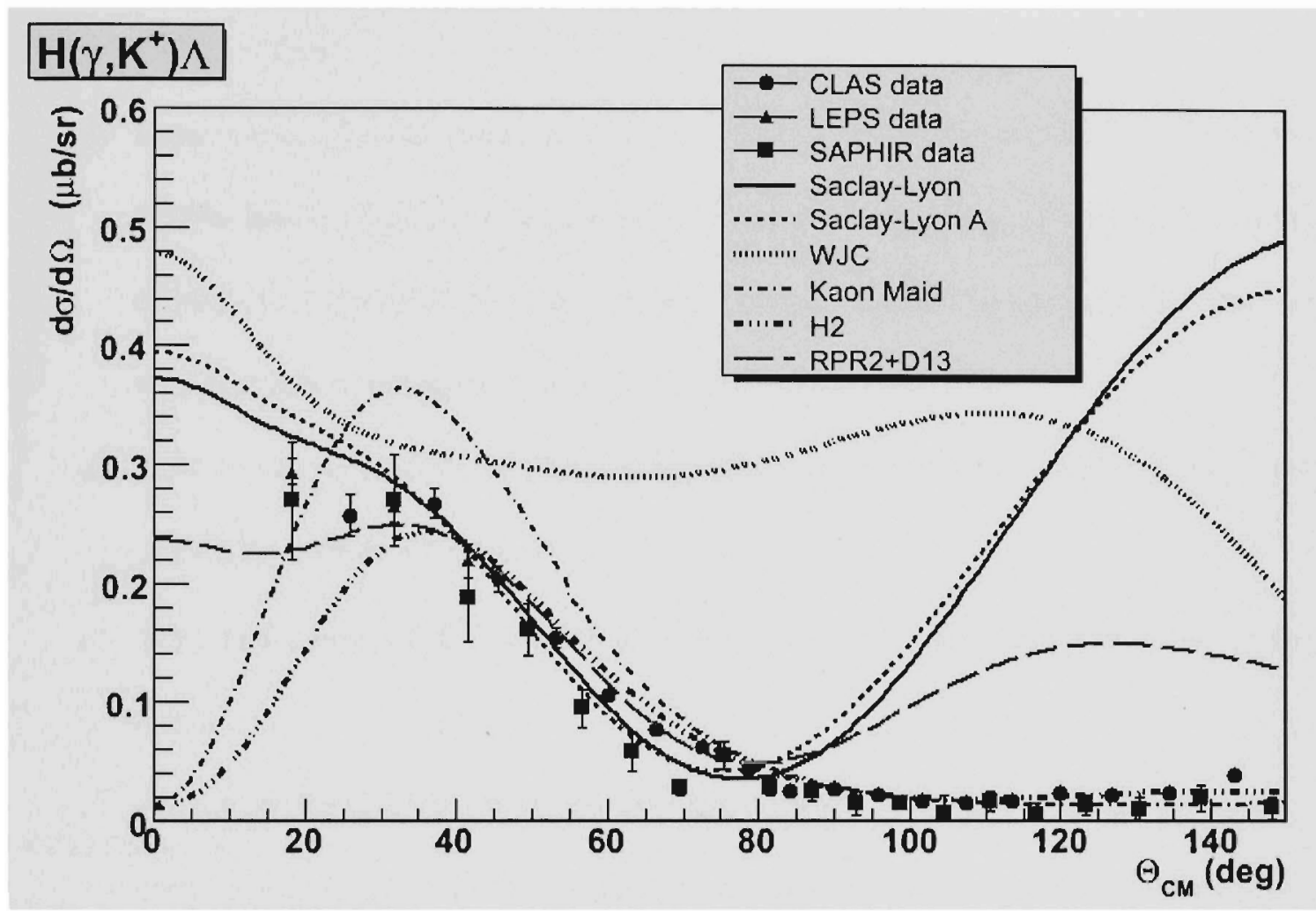

Figure 8: Isobaric and RPR2 models predictions compared to data [26], CLAS data from Bradford et al., [27].

Figure 8 shows predictions made by isobaric models and the RPR2 models for photoproduction at different kaon CM angles $\left(\theta_{C M}\right)$. The theoretical predictions differ at very forward angles and it is here where E94107 measures the cross section at 6 degrees. Although, E94107 is electroproduction, it is performed at very low $Q^{2} \sim 0.07(\mathrm{GeV} / \mathrm{c})^{2}$, which allows comparison of photoproduction and electroproduction reactions. By 
providing this important point, theoretical models can be discriminated. The main questions that this dissertation answers are:

a) What are the cross sections for ${ }^{1} \mathrm{H}\left(e, e^{\prime} K^{+}\right) \Lambda, \Sigma^{0}$ at $\theta_{K}=6^{\circ}, W=2.2 \mathrm{GeV}$ and $Q^{2} \sim$ $0.07(\mathrm{GeV} / \mathrm{c})^{2} ?$

b) Experiment E94-107 measures the cross section for electroproduction of hyperons that includes longitudinal amplitudes. What is the estimated transverse component of the electroproduction cross section? Which model better describes the data with the new experimental point included in Figure 8?

c) Does the cross section for photoproduction continue rising as the kaon angle goes to zero or is there a plateau?

d) What is the ratio of $\Sigma^{0} / \Lambda$ at low $Q^{2}$ ? How does this result compare to the existing data?

e) Which of the models describes the data at forward angles and can therefore be used in the analysis of the hypernuclear data without introducing an additional uncertainty?

Answers to the first four questions are important for understanding the dynamics of the elementary reaction process, while the last question is crucial for the hypernuclear calculations since the elementary amplitude scales the predictions. 
2.1 Overview of the Jefferson Lab

Experiment E94-107 was performed at the Thomas Jefferson National Accelerator Facility (also known as Jefferson Lab or JLab) located in Newport News, Virginia. The accelerator at Jefferson Lab can provide almost continuous electron beams to the three experimental halls: A, B and C (see Figure 9). The electron beams can reach up to energies of $5.7 \mathrm{GeV}$ with beam currents ranging from $10 \mathrm{nA}$ up to $200 \mu \mathrm{A}$.

Although the beam delivered by the accelerator is considered to be almost continuous it is actually made of pulses of electron packets accelerated by two linear accelerators (linacs) joined at their ends by recirculation arcs. The linacs consist of twenty cryomodules and each cryomodule has eight superconducting niobium cavities, which are kept at a temperature of $2 \mathrm{~K}$ during operations.

By shining a $780 \mathrm{~nm}$ laser on a GaAs photocathode, polarized electrons are obtained and accelerated up to $45 \mathrm{MeV}$ in the injector towards the first linac. At normal operations $0.4 \mathrm{GeV}$ is added to the beam each time it passes through a linac and after 5 passes the total energy reached would be $4.045 \mathrm{GeV}$. However, non-standard operation modes are available and energies close to $0.6 \mathrm{GeV}$ can be added to the beam per pass through a linac making a maximum of $5.7 \mathrm{GeV}$. There are 5 recirculation arcs at one end and 4 at the other end. The recirculation arcs have magnetic fields designed to bend the different energy beams through each arc, which before entering the next linac, get recombined again [32]. 
Radio waves with a frequency of $1497 \mathrm{MHz}$ are used in the cavities to accelerate the electron pulses, which are separated by a distant of $\mathrm{c} / 1497 \mathrm{MHz}=20 \mathrm{~cm}$, approximately. Normally one third of these pulses are sent to each hall at a frequency of one third of the accelerator frequency. Therefore, the frequency with which the pulses reach one experimental hall is $499 \mathrm{MHz}$. Subsequently, the time separation between pulses is approximately $1 / 499 \mathrm{MHz}=2 \mathrm{~ns}$. The energies used during this experiment were $3.77 \mathrm{GeV}$ and $3.96 \mathrm{GeV}$.

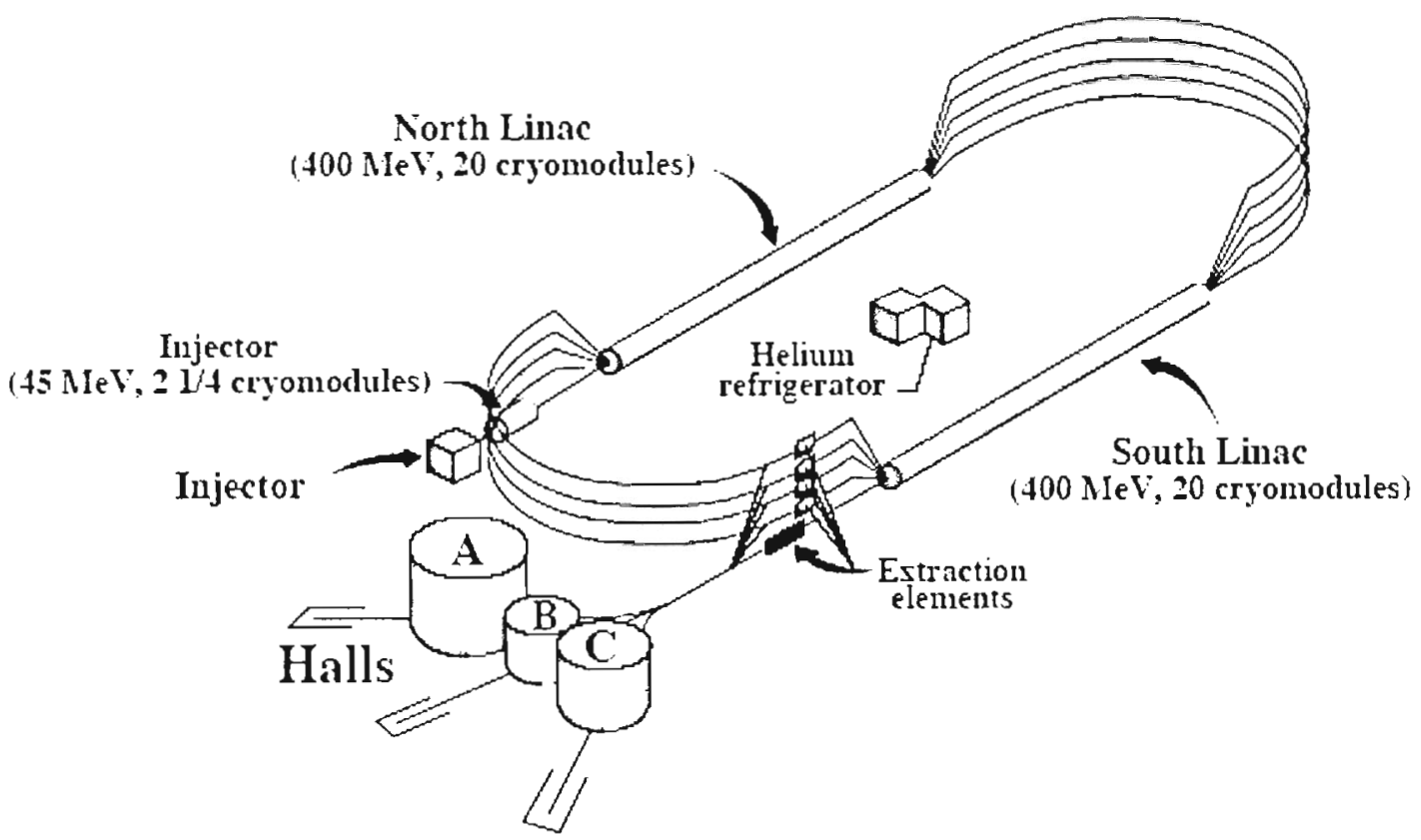

Figure 9: The accelerator at TJNAF [32].

The accelerator is now being upgraded and five new cryomodules are being added to each linac as well as a new arc added to one of the ends. The upgrade will allow the accelerator to reach a beam energy of up to $12 \mathrm{GeV}$. 


\subsection{The experimental Hall A}

Hall $\mathrm{A}$ is one of the three experimental halls where physics experiments are carried out. The electron beam enters the hall along the beam line. In order to monitor the beam current and beam position on the target there are two Beam Current Monitors (BCMs) and two Beam Position Monitors (BPMs) along the beam line just upstream of the target scattering chamber. After passing the BCMs and the BPMs the beam reaches the scattering chamber. Inside the scattering chamber the targets are located on a vertical ladder system, which allows remotely changing targets during data taking. A big portion of the beam gets through the target without interacting and continues beyond the scattering chamber to the final part of the beam line to end up in a shielded beam dump.

There are two spectrometers in the hall mounted on a system of rolling bearings so that they can be rotated to different scattering angles. The two spectrometers are designed with the same magnetic components and similar optical properties [32]. Normally, one of them, called the electron spectrometer (right) is used to measure the momentum and direction of the scattered electrons (also $\pi^{-}, \mu^{-} \ldots$ ) and the other, called the hadron spectrometer (left) is used to measure the momentum and direction of scattered positively charged particles $\left(p^{+}, \pi^{+}, K^{+} \ldots\right)$. These measurements in many cases are done in coincidence to detect particles from the same reaction.

At the end of each spectrometer there is a shielding house (also known as the detector hut) containing a detector package mounted on a support structure along with the electronics associated with the operation of the detectors for data collection. The purpose of the shielding houses is to protect the detectors and electronics by reducing the rates 
from particles or radiation that do not pass through the magnetic spectrometers. Figure 10 shows the interior of the Hall A.

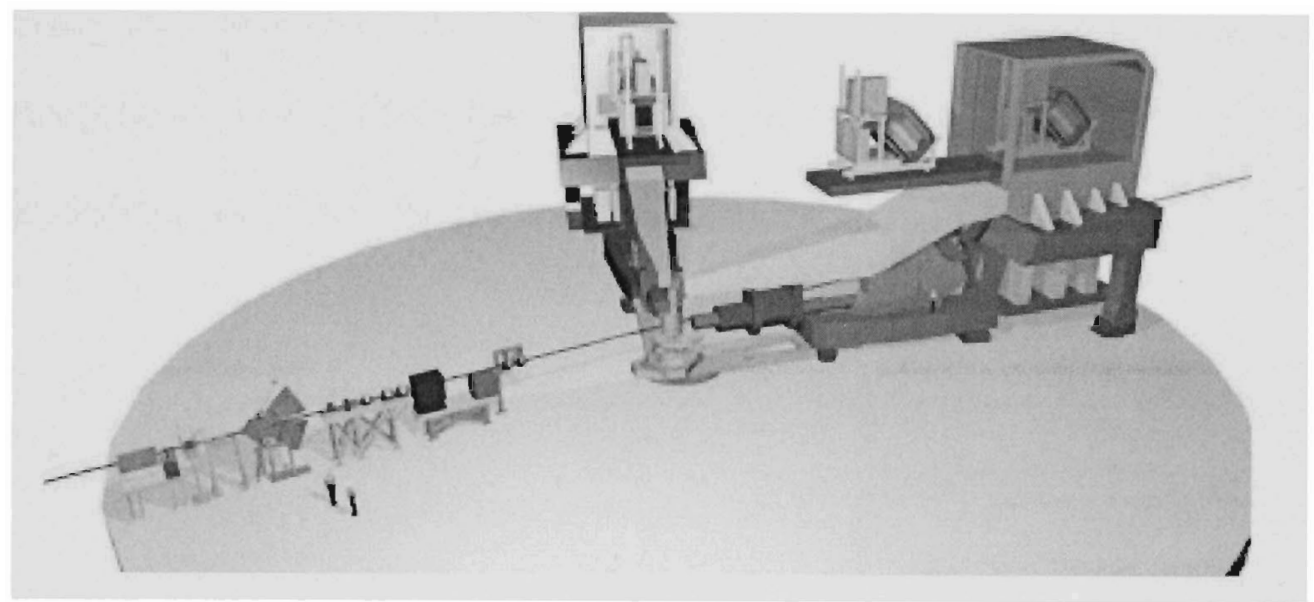

Figure 10: Experimental Hall A [33].

2.3 The beam line

\subsubsection{Beam energy}

There are two independent methods to measure the beam energy. The first method is done in the arc section of the beam line. To measure the energy, a known magnetic field is used to deflect the beam. The bending angle of the beam is $34.3^{\circ}$ and the momentum of the beam can be calculated by knowing the magnitude of the magnetic field and the net bend angle through the arc. Therefore, 


$$
p_{\text {beam }}=\frac{c}{\theta} \int \vec{B} \cdot d \vec{l}
$$

where $\mathrm{c}$ is the speed of light, $\vec{B}$ the magnetic field, and $\overrightarrow{d l}$ is the differential path length of the electrons [32]. The absolute energy resolution using the arc method is $1 \times 10^{-4} \mathrm{GeV}$. The energy used in E94-107 is $3.77 \mathrm{GeV}$, implying that in utilizing this method the energy uncertainty is about $0.4 \mathrm{MeV}$. In this method, the energy spread is measured at a high dispersion point, where the energy spread is:

$$
\frac{\sigma_{E}}{E_{\text {beam }}}=\frac{\sigma_{\delta}}{D}
$$

and $\sigma_{\delta}$ is the size of the beam due to dispersion $D$. Moreover, the total beam size is $\sigma_{\text {beam }}=\sqrt{\sigma_{\beta}{ }^{2}+\sigma_{\delta}{ }^{2}}$, where $\sigma_{\beta}$ is the beam betraton's size. However, for high dispersion point measurements the contribution of $\sigma_{\beta}$ to the beam size can be ignored, so with $\sigma_{\text {beam }}>\sigma_{\delta}$ in Equation (24). Then the upper limit of the energy spread is

$$
\frac{\sigma_{E}}{E_{\text {beam }}}<\frac{\sigma_{\text {beam }}}{D}
$$

In E94-107 the beamline optics had the dispersion $D=4 \mathrm{~m}$ and the energy spread was $3 \times 10^{-5}[34]$. 
Another method to measure the beam energy is called the "ep" method. This method is based on the kinematics of elastic scattering of the electron beam off a proton target. To do this measurement two sets of detectors are placed symmetrically about the incident beam direction. Therefore, at either side of the beam the scattered electron and the recoiling protons are detected and, by the two-body kinematics, the energy is

$$
E=M_{p} \frac{\cos \left(\theta_{e}\right)+\frac{\sin \left(\theta_{e}\right)}{\tan \left(\theta_{p}\right)}-1}{1-\cos \left(\theta_{p}\right)},
$$

where $\theta_{e}$ and $\theta_{p}$ are the scattering angles of the electrons and protons from the incident electron beam direction and $M_{p}$ is the mass of the proton [35]. The accuracy of the measurement in the "ep" method is up to $\frac{\delta E}{E}=2 \times 10^{-4}$. The agreement between the two methods (relative uncertainty) is about $3 \times 10^{-4}$.

In addition, in order to have a complete characterization of the beam energy it is also important to measure the beam energy spread as well as the centroid. These measurements are performed by two methods as well. One of the methods is Optical Transition Radiation (OTR) and it consists of a $0.3 \mu \mathrm{m}$ thin carbon foil where the transverse beam size is measured by imaging the optical transition radiation on a CCD camera and analyzing the horizontal and vertical projections. The other method is known as the Synchrotron Light Monitor and it uses a synchrotron light interferometer pattern to measure the transverse beam size [36]. 


\subsubsection{The beam current}

The precise measurement of the current is needed in order to measure cross sections normalized to the luminosity. To measure the beam current a DC current transformer monitor (DCT) is used (Unser monitor). Since the DC beam current provides no time varying flux component to generate a signal by magnetic induction, an AC flux component is "brought to the beam" via the action of a magnetic modulator circuit. The operation of a magnetic modulator, and hence a DCT, is based on the non-linear characteristics of high-quality tape-wound magnetic cores [37]. The Unser monitor is designed for non-destructive beam current measurements and it provides an absolute reference. The Unser monitor can also be calibrated by setting a known current in a wire that passes through the Unser. The nominal output is of $4 \mathrm{mV} / \mu \mathrm{A}$. However, the Unser monitor tends to drift after several minutes so it is normally used only for calibration purposes.

To monitor the current continuously, two other BCMs are used. They are two resonant cavities located in the beam line about $24.5 \mathrm{~m}$ upstream of the target. These BCMs are basically cylindrical resonant cavities made out of stainless steel with a diameter of $15.18 \mathrm{~cm}$ and a length of $15.24 \mathrm{~cm}$. Two loop coaxial antennas are inside each cavity and their positions are where the $H$ field reaches its largest magnitude. The resonant frequency of the cavities is adjusted to the frequency of the beam pulses, that is to say $499 \mathrm{MHz}$. Consequently, the output voltages levels are proportional to be beam current. An analog DC voltage level is obtained from converting the RMS output from the cavities. However, its response is not linear for currents below $5 \mu \mathrm{A}$. Therefore, to 
extend the non-linear region of this response, the signals are amplified by 3 and by 10 . Furthermore, by knowing the time duration of a run the amount of charge can be measured and then, the charge data is stored in the Experimental Physics and Industrial Control System (EPICS) data stream [38]. Figure 11 shows the charge ratio of the two amplified BCM signals with respect to the non-amplified one.

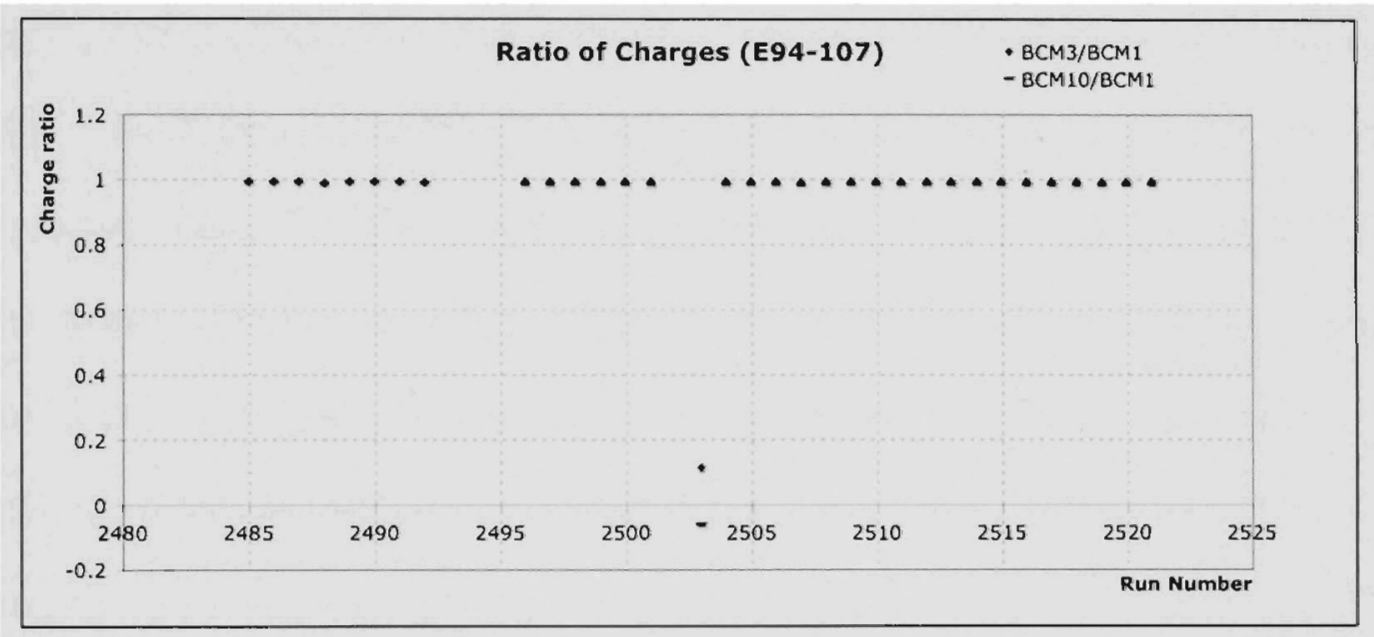

Figure 11: Charge ratio of the two amplified signals to the non-amplified signal for experiment E94-107.

\subsubsection{Beam position monitor and raster}

Two BPM devices placed upstream of the target at $1.286 \mathrm{~m}$ and $7.524 \mathrm{~m}$ are used to monitor the beam position in the horizontal $(\mathrm{X})$ and vertical $(\mathrm{Y})$ directions. Each BPM has two 4-wire antennas arrays of open-ended thin wire strip lines tuned to the fundamental radio frequency of the beam. For currents above $1 \mu \mathrm{A}$ the beam position can be determined within a precision of less than $100 \mu \mathrm{m}$. Adjacent to each of the BPMs are 
located the 'superharp' scanners at $7.353 \mathrm{~m}$ and at $1.122 \mathrm{~m}$ upstream, which are used to calibrated the absolute position of the BPMs [38]. The information obtained by the BPMs is recorded into the EPICS data stream every 3 to 4 seconds with a record of the time at that moment.

The superharp can measure not only the absolute position of the beam centroid but also the profile or spatial extent of the beam. To do this, the superharp has one tungsten wire bent twice to give 3 straight sections, two of which measure the beam profile in the $\mathrm{Y}$ direction and the third in the $\mathrm{X}$ direction. These wires are mounted into a fork-shaped frame. Therefore, when the frame is moved towards the beam making the wires cross the beam, secondary electrons are emitted producing a signal which is detected and amplified. The positions of the wires are well known allowing reliable absolute positions and widths of the beam in the $\mathrm{X}$ and $\mathrm{Y}$ directions as well. There is an analog-to-digital converter (ADC) that measures the signal on the wires and also a position encoder which measures the position of the ladder that holds the wires. The proper combination of this information determines the position and profile of the beam in the coordinate system of the hall. The beam position is typically obtained with an accuracy of about $10 \mu \mathrm{m}$ and the width of the beam is about $100 \mu \mathrm{m}$ (FWHM).

When data are collected, an intense beam is used to maximize the luminosity. However, an intense beam can destroy the target. To prevent the target from being damaged by the beam, a raster system is used. A raster system is located $24 \mathrm{~m}$ upstream of the target. The raster system sweeps the beam to cover an area over the target instead of a fixed point, spreading the heat from the impact of the beam over the covered area and preventing the target from large, local-density fluctuations for liquid targets. The 
raster has $\mathrm{X}$ - and $\mathrm{Y}$-direction air-core dipoles and they can operate in two different modes, sinusoidal- and amplitude-modulated. The sinusoidal-mode uses purely sine waves with a relative phase of $90^{\circ}$. In the amplitude modulated mode the $\mathrm{X}$ and $\mathrm{Y}$ magnets are driven at a frequency between $17.7 \mathrm{KHz}$ and $24.4 \mathrm{KHz}$ with a difference of phase of $90^{\circ}$ producing a circular pattern. By changing the radius of the pattern a uniform distribution of the beam is obtained. The raster of the beam in experiment E94-107 had a rectangular shape of approximately $2 \mathrm{~mm} \times 2 \mathrm{~mm}$ shown in the following Figure 12 .
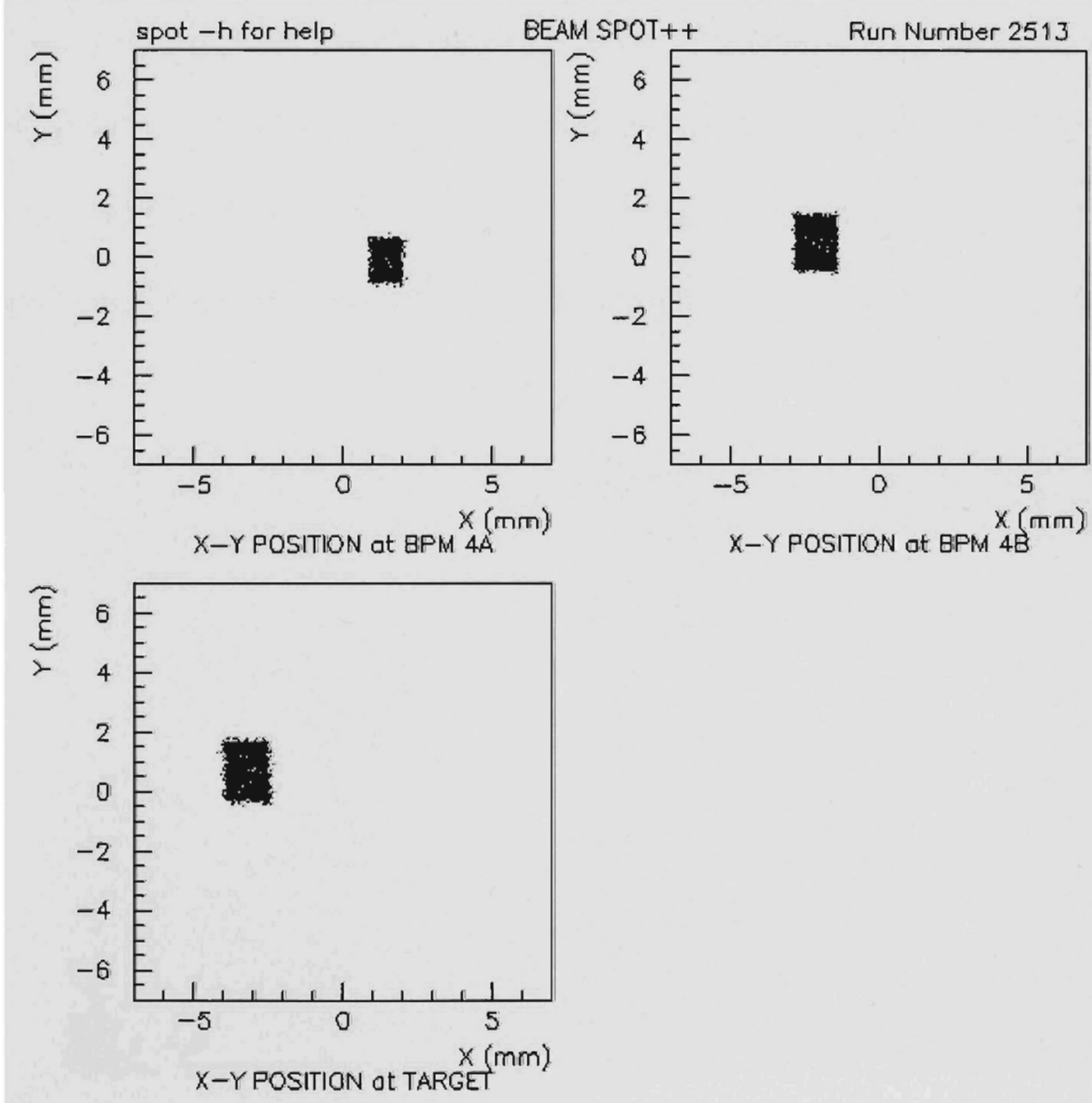

Figure 12: Rastered beam size and position in experiment E94-107. 


\subsection{Target system}

The design of the target system allows placing different targets in the same ladder, so it is possible to remotely change targets during the experiment. The different targets that can be selected are solid flat targets and cryotargets (liquid). The targets are mounted on a vertical ladder. The ladder can be moved up and down remotely through a DC stepping motor. The accuracy of the relative position of the targets with respect to the beam line is about 0.020 inches. The accuracy in moving the target from one position to another and moving it back is about 0.006 inches. The solid flat target ladder is usually mounted under a system that holds the containers with the cryotargets. The cryotarget chamber has a vertical assembly of long $(\sim 15 \mathrm{~cm})$ horizontal and short $(\sim 4 \mathrm{~cm})$ horizontal containers to hold liquid hydrogen $\left(\mathrm{LH}_{2}\right)$, Deuterium $\left(\mathrm{LD}_{2}\right)$, Helium-4 and others.
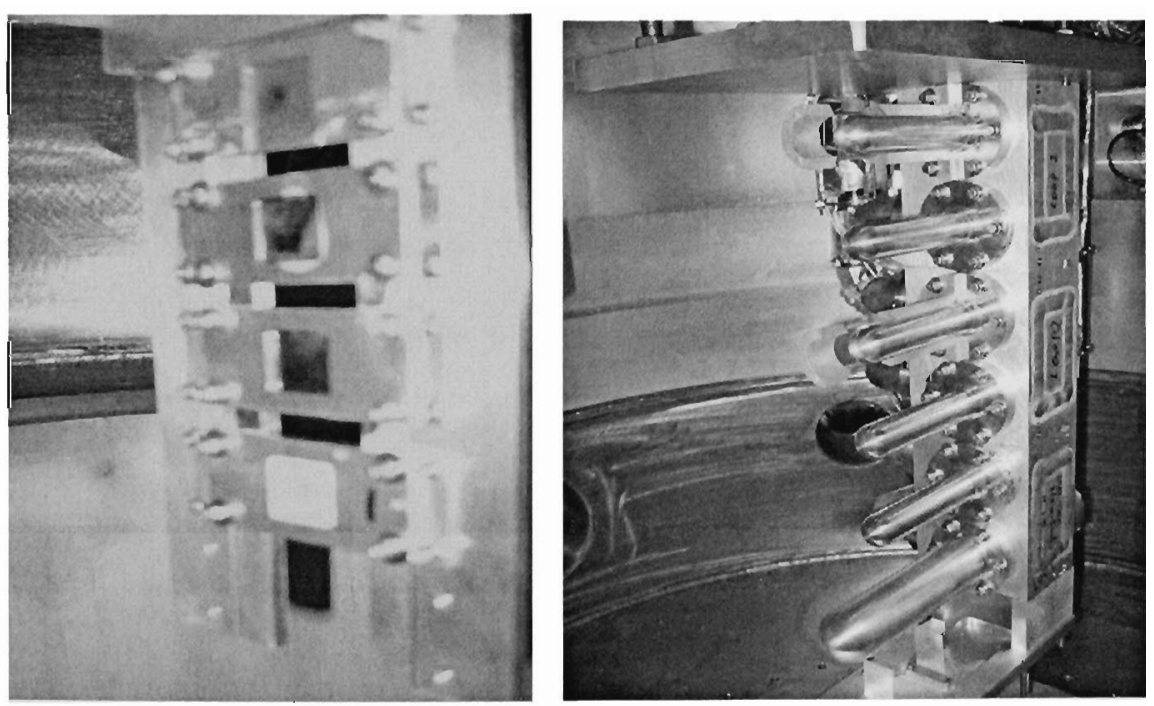

Figure 13: Pictures of the target ladder (left) and cryotarget system (right). 
The complete target system is installed inside the scattering chamber along with sub-systems for cooling, gas handling, temperature and pressure monitoring. All these variables are monitored remotely from the counting house (control room). Among the solid targets we had ${ }^{12} \mathrm{C}$ (thin $\sim 50 \mathrm{mg} / \mathrm{cm}^{2}$ and thick $100 \mathrm{mg} / \mathrm{cm}^{2}$ ), which are used for optics studies. Other solid targets were $\mathrm{BeO}$ and $\mathrm{Ta}$. $\mathrm{BeO}$ is used to have a visual position of the beam over the target since it glows when the electron beam impacts it and $\mathrm{Ta}$ is used for commissioning, since it has a large atomic number and it is useful for the scattering of electrons with a minimum recoiling momentum. Dummy targets are also availble, which consist of thick aluminum walls to simulate the cryotarget containers. These dummy targets have thickness 10 times larger than those of the cryotarget containers for the purpose of collecting data in less time. They also have the same radiation length as a full target. Once the information for a dummy target is obtained, a subtraction of the background it produces can be done from the cryotarget that contains the target itself $\left(\mathrm{LH}_{2}\right.$ or other) plus the walls of the aluminum container of the cryotarget [39]. The scattering chamber that has the purpose of protecting the targets mechanically and thermally has a cylindrical shape with an inner radius of about $100 \mathrm{~cm}$ and a height of $90 \mathrm{~cm}$. The thickness of the wall of the scattering chamber is about $5 \mathrm{~cm}$. Openings for entrance and exit of the beam are at both ends of the chamber. The scattered particles exit the scattering chamber through very thin aluminum windows. Each aluminum window is about $18 \mathrm{~cm}$ high and the thickness of the aluminum walls is about $40 \mu \mathrm{m}$.

The cryotargets are installed with loops that contain a cooling system, a heat exchanger, and a fan system that it set to run at $60 \mathrm{~Hz}$. All this equipment is meticulously 
organized to keep the target at a very low temperature and in a narrow range of temperatures. In E94107, the $\mathrm{LH}_{2}$ target was kept at $19 \mathrm{~K}$.

In E94-107, the solid targets were also used for the different optics studies and commissioning. The $\mathrm{LH}_{2}$ (April 2004) and the waterfall (June 2005) targets were used for the production data. The waterfall target was installed in 2005 , for the second part of the experiment. Technical reasons did not allow having the cryotarget system and the waterfall target simultaneously. The waterfall target was installed in the space occupied by the cryotarget system for the second part of the experiment. The waterfall target has the special feature to allow us to have two different kinds of targets at the same time: oxygen and hydrogen. In other words, we look into the two nuclear reactions: ${ }^{1} \mathrm{H}\left(e, e^{\prime} K^{+}\right) \Lambda, \Sigma^{0}$ and ${ }^{16} \mathrm{O}\left(e, e^{\prime} K^{+}\right){ }_{\Lambda}^{16} \mathrm{~N}$ simultaneously. The former represents the elementary production of hyperons and the latter is for hypernuclear spectroscopy studies of the ${ }_{\Lambda}^{16} \mathrm{~N}$ hypernucleus. This dissertation is focused on the elementary production of hyperons at low momentum transfer. The data used in this dissertation comes from the first part of the experiment E94-107 in which a $\mathrm{LH}_{2}$ cryotarget was used. The study of the elementary reaction is also used to calibrate the hypernuclear spectroscopy study of ${ }_{\Lambda}^{16} \mathrm{~N}$, whose data is obtained in the second part of E94107 using the waterfall target.

The waterfall target consists of water falling with thickness controlled by a system, which through a gear pump magnetically coupled to a DC motor, used to produce a stable water film. By changing the speed of the pump, monitored with a tachometer the thickness can be changed. A cooler is used to control the temperature and the thickness is measured by elastic scattering of electrons off hydrogen [40]. 


\subsection{High resolution spectrometers}

The two high resolution spectrometers (HRSs) each have at their entrances two superconducting focusing quadrupole magnets Q1 and Q2 that are followed by a dispersing $6.6 \mathrm{~m}$ magnetic dipole, which bends the particle trajectories vertically upwards, with a nominal bending angle of $45^{\circ}$. The magnetic dipoles use a field gradient to further focus the particles that pass through them. A third superconducting quadrupole magnet Q3 does a final focusing before particles get into the detector hut. Therefore, both HRSs have a QQDQ configuration, $12.5^{\circ}$ being the minimum forward angle they can go and $125^{\circ}$ as the maximum. The dipole magnetic field can be set at a central-momentum value ranging from $0.3 \mathrm{GeV} / \mathrm{c}$ to $4 \mathrm{GeV} / \mathrm{c}$. The central-ray particle trajectory is the reference for the symmetry plane of the spectrometer magnets. The optical length of the central ray from the target to the exit window of the spectrometers is $23.4 \mathrm{~m}$ (see Figure 14). All these features constitute the standard configuration and the parameters that characterize the HRSs are shown in Table 5 [32]: 
HRS General Characteristics:

Momentum range: $0.3-4.0 \mathrm{GeV} / \mathrm{c}$

Configuration: QQDQ

Bending angle: $45^{\circ}$

Optical Length: $23.4 \mathrm{~m}$

Momentum Acceptance: $\pm 4.5 \%$

Dispersion at the focus(D): $11.9 \mathrm{~m}$ (without septum), $12.4 \mathrm{~m}$ (with septum).

Momentum Resolution $\delta p / p$ (FWHM): $1 \times 10^{-4}$

Horizontal Angular Acceptance: $\pm 30 \mathrm{mrad}$

Vertical Angular Acceptance: $\pm 60 \mathrm{mrad}$

Solid Angle at $\delta p / p=0, y_{0}=0: 6 \mathrm{msr}$

Horizontal Angular Resolution: $0.5 \mathrm{mrad}$

Vertical Angular Resolution: $1.0 \mathrm{mrad}$

Angular Determination Accuracy: $0.1 \mathrm{mrad}$

Table 5: General characteristics of the HRSs of the Hall A. 


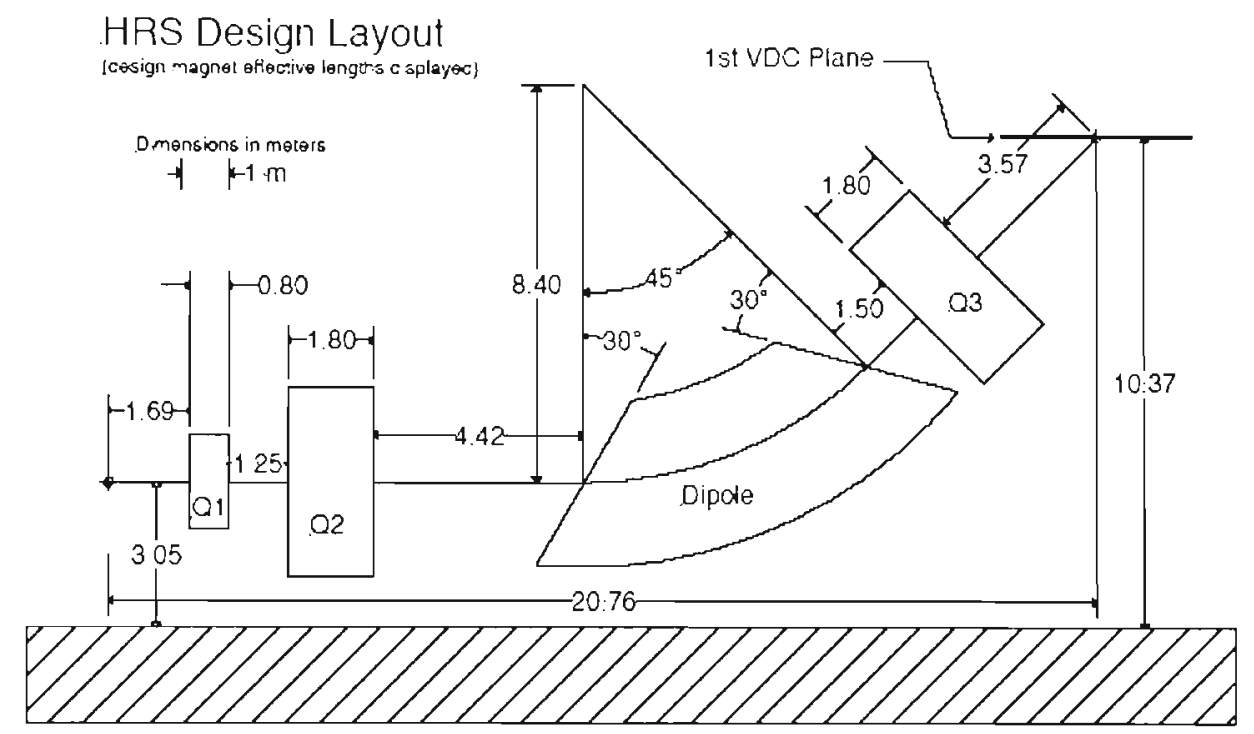

Figure 14: Profile view of the spectrometers in Hall A [32].

On top of the HRSs are the detector huts with their detector packages that can be moved in and out for maintenance and access. Each detector package contains some standard detectors and some specific detectors designed for this specific experiment. The detector configurations for the experiment E94-107 are shown in Tables 6 and 7 and Figures 15 and 16:

Hadron Spectrometer (HRS-L)

VDC: Two Vertical Drift Chambers

S1: Trigger Scintillator Counters

Al: $1^{\text {st }}$ Aerogel Counter

A2: $2^{\text {nd }}$ Aerogel Counter

RICH: Ring Imaging Cherenkhov Detector

S2: Trigger Scintillator Counter

Table 6: Hadron arm detector configuration for E94-107. 
In experiment E94-107 The HRS-L was configured to analyze positively charged particles like $K^{+}, \pi^{+}$and $p^{+}$. The VDCs provide information on momentum and position of particles while the two layers of scintillators S1 and S2 provide information on the time of flight. A1 and A2 discriminate $K^{+}$from $\pi^{+}$and $p^{+}$background. The RICH detector enhances the particle identification (PID) done by Al and A2. More details are discussed in Section 2.6.

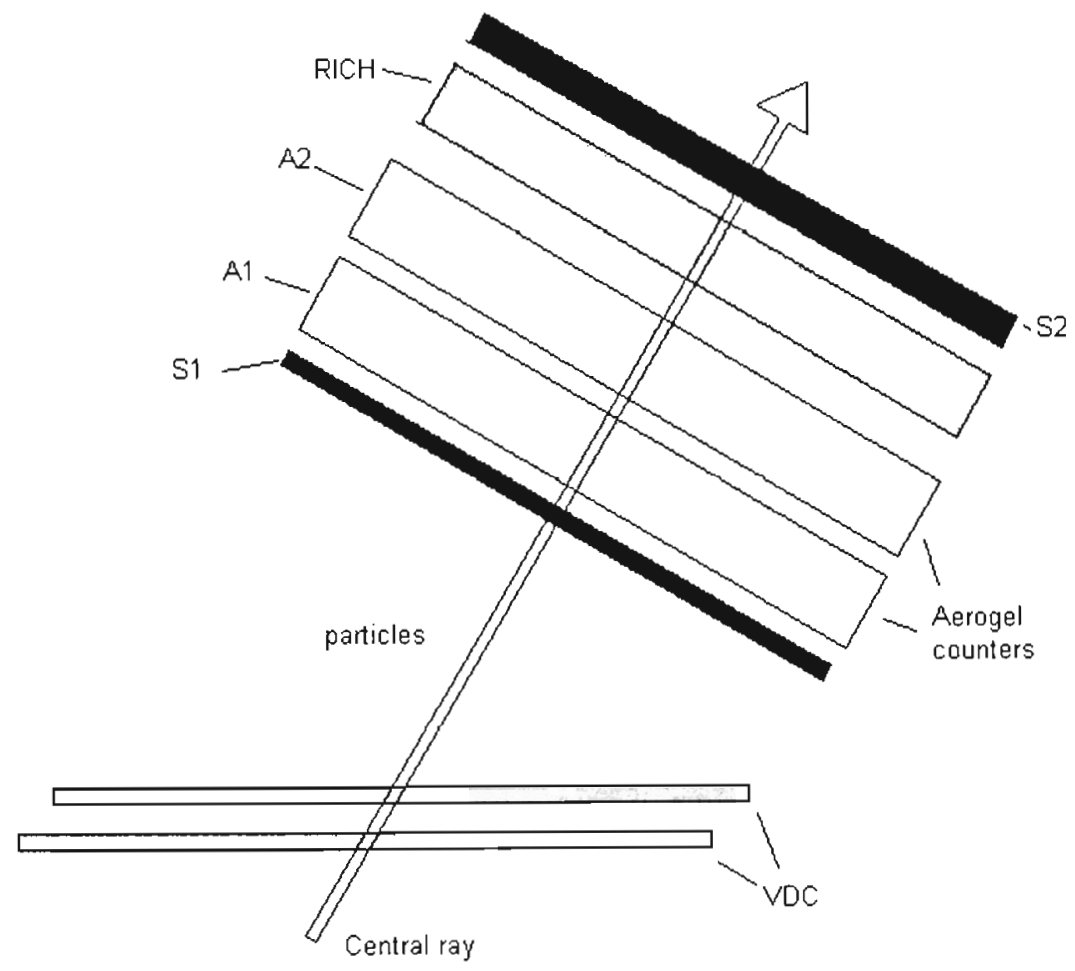

Figure 15: Configuration of the detector package in the HRS-L. 
The right HRS has the following configuration:

\begin{tabular}{l} 
Electron Spectrometer (HRS-R): \\
\hline VDC: Two Vertical Drift Wire Chambers \\
S1: Trigger Scintillator Counters \\
CER: $\mathrm{CO}_{2}$ Gas Cherenkhov counter \\
S2: Trigger Scintillator Counters \\
PS: Preshower Counters \\
SH: Total Shower Counters
\end{tabular}

Table 7: Electron arm detector configuration for E94-107.

In experiment E94-107 The HRS-R was configured to analyze negatively charged particles as $e^{-}$and $\pi^{-}$. The VDCs provide information on momentum and position of particles while the two layers of scintillators S1 and S2 provide information of the time of flight. The $\mathrm{CO}_{2}$ Gas Cherenkhov counter (CER) discriminates $e^{-}$from $\pi^{-}$background. The preshower (PS) and shower (SH) detectors enhances the particle identification (PID) done by CER. More details are discussed in Section 2.6. 


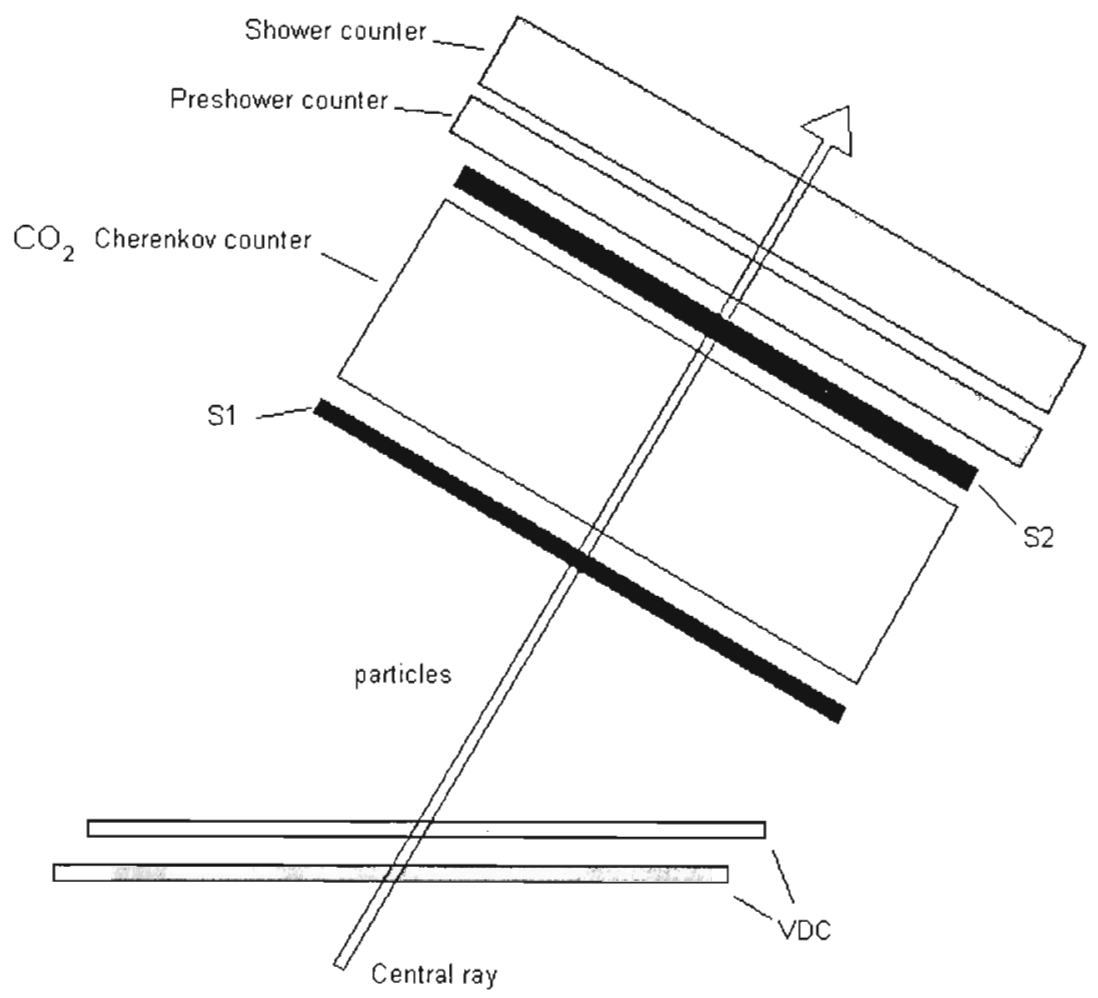

Figure 16: Configuration of the detector package in the HRS_R.

\subsubsection{Septum magnets}

Experiment E94-107 required very forward scattering angles of $\sim 6^{\circ}$. Since the HRSs cannot go further than $12.5^{\circ}$ a new pair of magnets were inserted in front of the two spectrometers and after the scattering chamber to be able to horizontally bend trajectories of scattered particles at forward angles $\left(\sim 6^{0}\right)$ towards the entrance of the spectrometers located at $12.5^{\circ}$ without significantly affecting the optics and momentum resolution of the two HRSs and in this way ensure good resolution in the missing mass spectra (see Figure 17). An important consideration in the design of the septa is maximizing the angular acceptance to match the solid angle of the spectrometers of around $4.5 \mathrm{msr}$. 


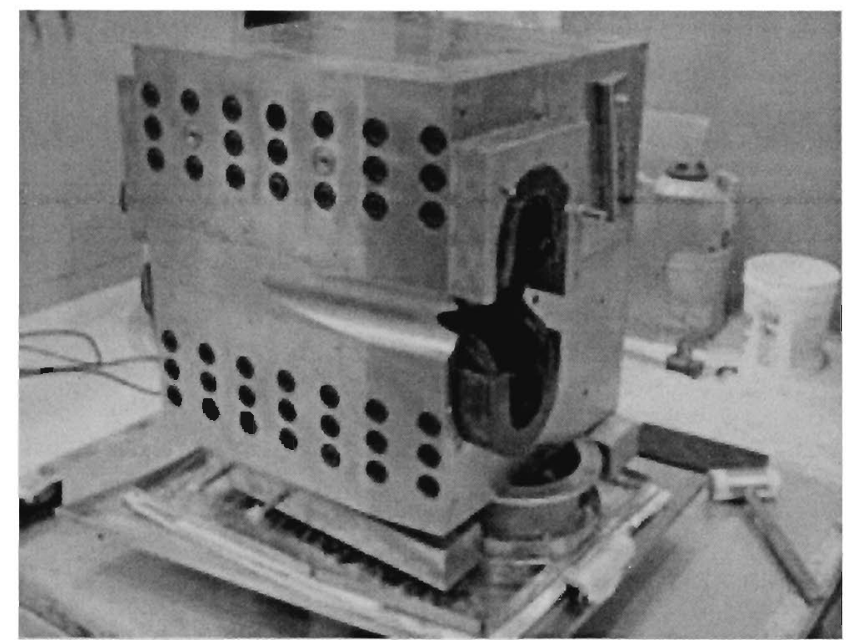

Figure 17: Picture of one of the septum magnets during fabrication.

The design of the septa allows complete angular coverage over the full range from $6^{0}$ to $12.5^{0}$ to smoothly overlap the standard HRS minimum angle for all momenta up to 4 $\mathrm{GeV} / \mathrm{c}$. In particular, there are two important parameters, they are the distance (d) that the target chamber was moved upstream in order to provide enough space to insert the septa, $80 \mathrm{~cm}$, and the distance $(\mathrm{T})$ from the septum gap to the beam line which was minimized to be $2.5 \mathrm{~cm}$ (see Figure 18) [41]. The optics was studied and the results were as expected and are described in Section 3.2.

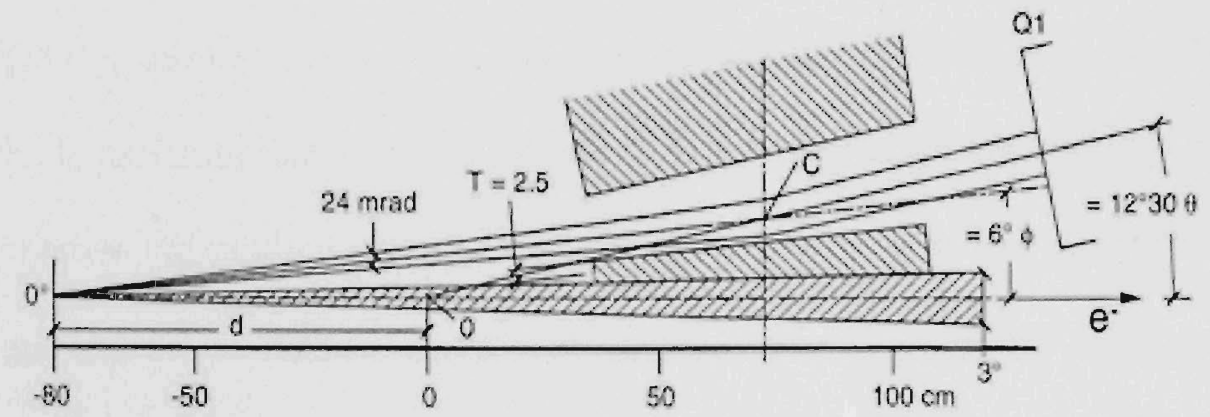

Figure 18: Layout of the septum magnet insertion [41]. 


\subsection{Detector stack packages}

The two high-resolution spectrometers are very similar except for some detectors used for PID. The position of the detectors in the detector stacks is shown in Figure 15 and Figure 16. The detectors used in experiment E94107 are listed in Tables 6 and 7.

\subsubsection{Scintillator counters}

Each HRS uses two layers of scintillators called S1 and S2. These layers are made of thin plastic scintillators (polyvinyl toluene, PVT) to minimize hadron absorption. They are separated $2 \mathrm{~m}$ along the flight path. The S1 consists of six overlapping paddles with dimensions $36 \mathrm{~cm} \times 30 \mathrm{~cm} \times 0.5 \mathrm{~cm}$ and the $\mathrm{S} 2$ consists of six paddles with dimensions $60 \mathrm{~cm} \times 37 \mathrm{~cm} \times 0.5 \mathrm{~cm}$. At both ends of each counter (paddle) there are photomultiplier tubes (PMT) attached through light couplers. The PMTs are Burle 8575 and their time resolution is $200 \mathrm{ps}$ per plane [32].

The set of two layers S1 and S2 in each detector stack allows measuring the time it takes for charged particles to travel the distance in between. Therefore, using the time of flight (TOF), the velocity of particles can be measured. In order to prevent light leakage, the scintillators are wrapped in one layer of aluminum foil and two layers of Tedlar (polyvinyl fluoride). The active area for $\mathrm{S} 1$ is $170 \mathrm{~cm} \times 35 \mathrm{~cm}$ and for $\mathrm{S} 2$ is 220 $\mathrm{cm} \times 54 \mathrm{~cm}$ and the scintillator paddles overlap by $5 \mathrm{~mm}$ to maximize coverage of the area through which particles go (see Figure 19). The first scintillator plane (S1) is located at $1.5 \mathrm{~m}$ from the center of the first vertical drift chamber (VDC) and the second 
scintillator plane (S2) is located at $3.5 \mathrm{~m}$ from the center of the first plane of the VDC.

Triggers are generated by the PMT signals from the scintillator planes and are sent to all other detectors and data acquisition system.

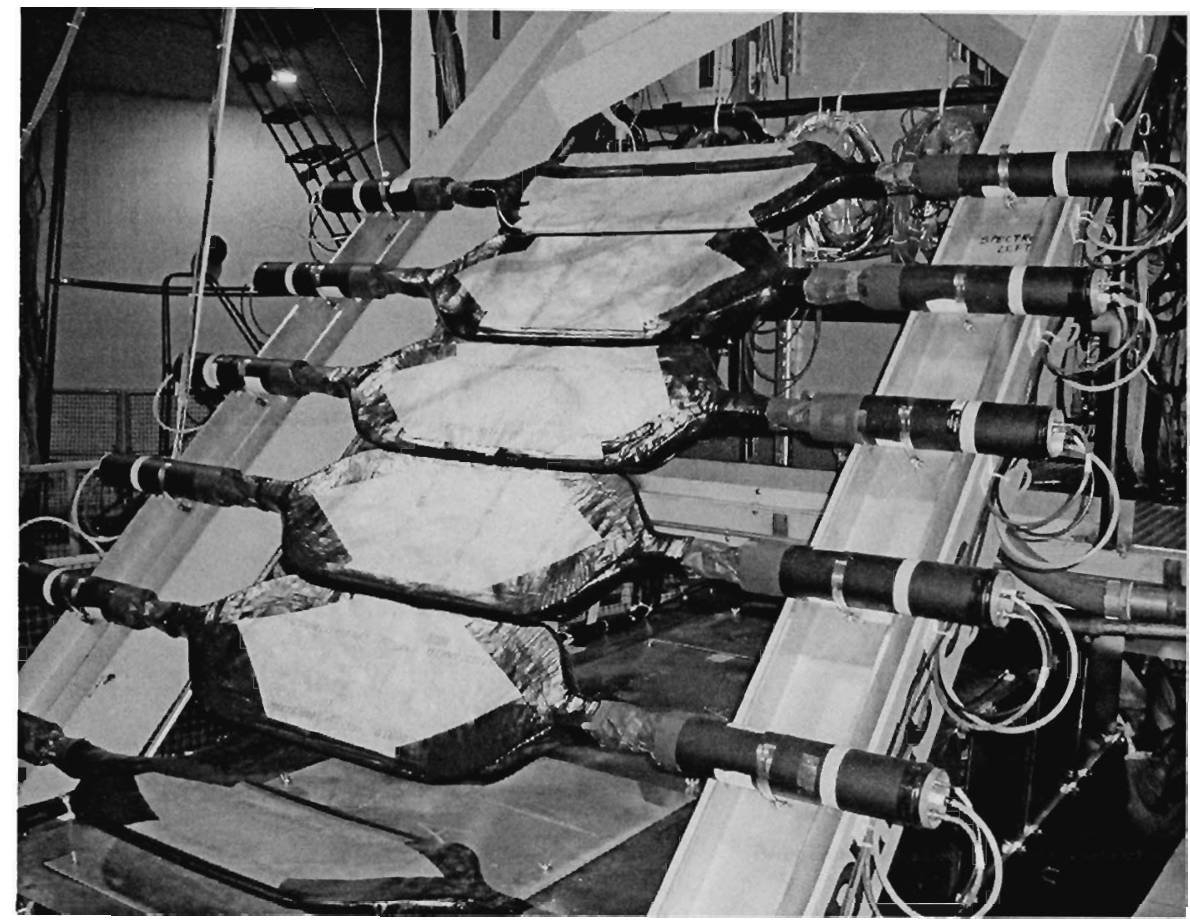

Figure 19: View of S1 looking upstream.

\subsubsection{Vertical drift chambers (VDC)}

The VDCs are the HRS tracking detectors. They provide measurements of position and angle for both electrons (HRS-R) and hadrons (HRS-L) at the focal planes of the respective spectrometers $[32,42]$. The VDC consists of two planes separated by a distance of $33.5 \mathrm{~cm}$ in each HRS detector stack. However, because of the $45^{\circ}$ angle of the central ray, the distance along the central ray is about $47.4 \mathrm{~cm}$. Each of the VDC planes 
are composed of two wire planes $U$ and $V$. The distance between the two $U$ and $V$ planes is $26 \mathrm{~mm}$. The wires of the $U$ plane are perpendicular to the wires of the $\mathrm{V}$ plane. Both $\mathrm{U}$ and $\mathrm{V}$. wires make an angle of $45^{\circ}$ with the dispersive and transverse directions in the spectrometer. The VDC wires are in a chamber filled with a mixture of gases: Argon $(62 \%)$ and Ethane (38\%). The gas mixture flows through the chambers at 10 liters/hour.

Details are shown in Figure 20.
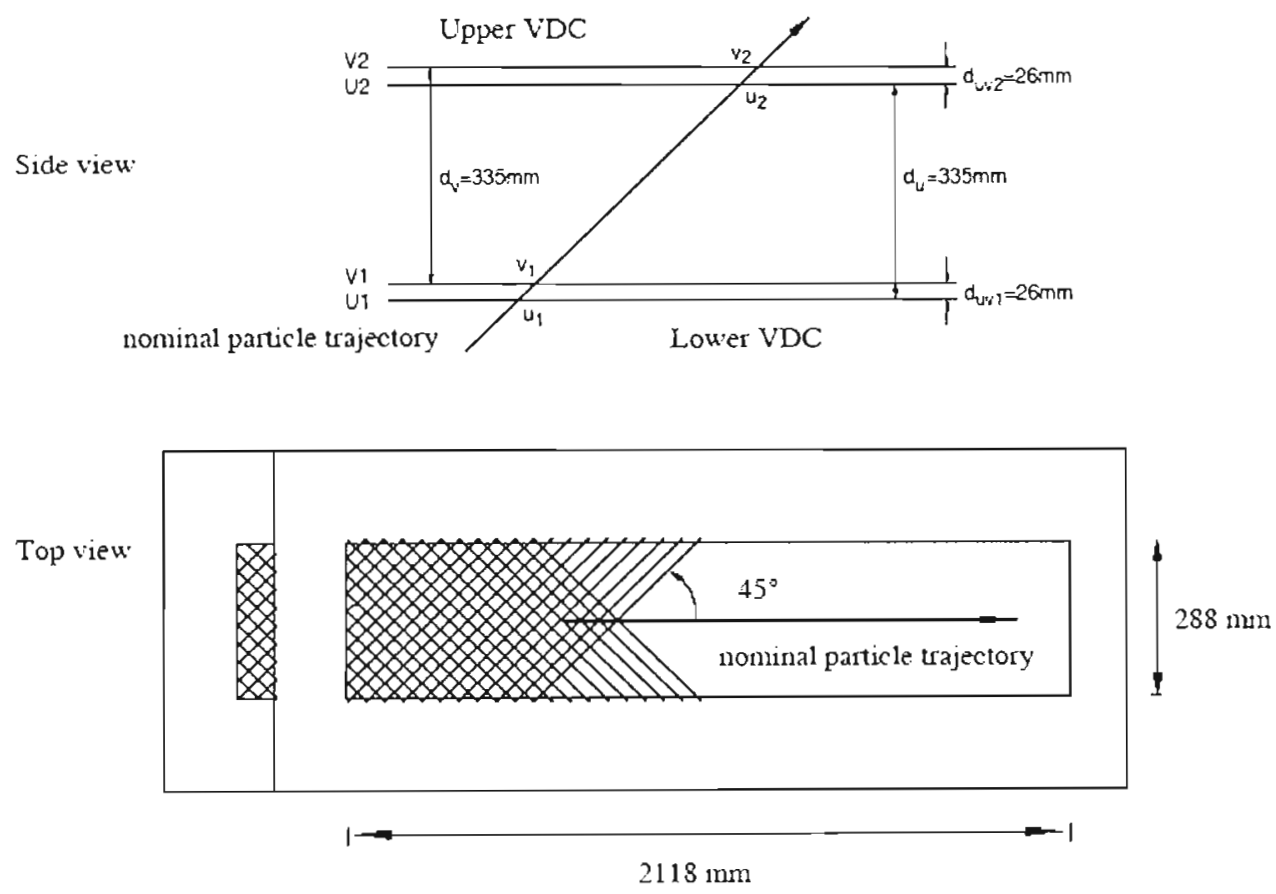

Figure 20: Details of the vertical drift chambers [32].

As charged particles pass through the gas chamber they produce ionization. In addition, each VDC has three high voltage planes (cathodes) at $-4 \mathrm{KV}$. The cathode planes 
and the $\mathrm{U}$ and $\mathrm{V}$ wire planes are parallel and alternated so each wire plane is in between two cathode planes. The resulting electric field configuration is shown in Figure 21.

When electrons are produced by ionization they drift to the wires. That time is measured by a Time to Digital Converter (TDC), which is started by the triggered wire and stopped by a signal from the trigger supervisor. The trigger supervisor is the electronic device that controls the Hall A data acquisition system.

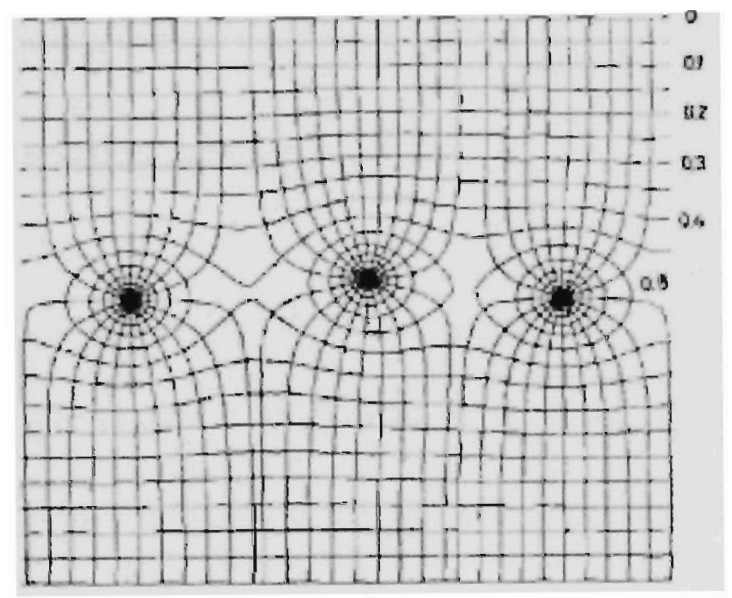

Figure 21: Electric field and equipotential lines inside the VDC [43].

Figure 21 shows a diagram of the distribution of electric field lines and equipotential lines in a VDC making most of the drift of the electrons produced by ionization along the perpendicular direction of the planes. If the drift distance and the drift time through the TDCs are obtained then the drift velocity of the electron in the gas mixture can be determined. The drift chamber TDCs measure the time that the wire detected the electrons created by the ionization of the chamber gas relative to the time of 
the trigger. Then using the scintillator TDCs to determine the time that the particle passed through the focal plane (relative to the trigger), the time it took for the electrons created by the ionizing particles to drift to the wire can be determined. The drift time is converted into a drift distance and, taking into account the distance from all the wires that fired when a particle passed through the VDC, the trajectory of the particle can be obtained. Position is determined with a resolution of $225 \mu \mathrm{m}$ (FWHM) and the angular resolution is approximately $0.3 \mathrm{mrad}$ (FWHM) [44].

\subsubsection{Gas threshold Cherenkov detector}

A $\mathrm{CO}_{2}$ gas threshold Cherenkov detector was used in the right HRS to distinguish electrons from negative pions and it is located in between the S1 and S2 scintillator planes in the HRS-R detector stack as shown in Figure 16. The detector contains $\mathrm{CO}_{2}$ at atmospheric pressure with an index of refraction $(n)$ of 1.00041 . The length of the particle path in the gas radiator is about $130 \mathrm{~cm}$. The threshold speed of particles is $\beta_{t h}=1 / n=0.999590$. Consequently, the threshold momentum given by $p_{t h}=\frac{m_{o} c}{\sqrt{1-\beta^{2}}}$, with $m_{o}$ as the rest mass, is $4.8 \mathrm{GeV} / \mathrm{c}$ for negative pions and $17 \mathrm{MeV} / \mathrm{c}$ for electrons [45]. Therefore, the $\mathrm{CO}_{2}$ gas threshold Cherenkov detector with a momentum range from 0.30 $\mathrm{GeV} / \mathrm{c}$ to $4.0 \mathrm{GeV} / \mathrm{c}$ is an excellent tool for particle identification (PID) as described in Chapter 4.5. 


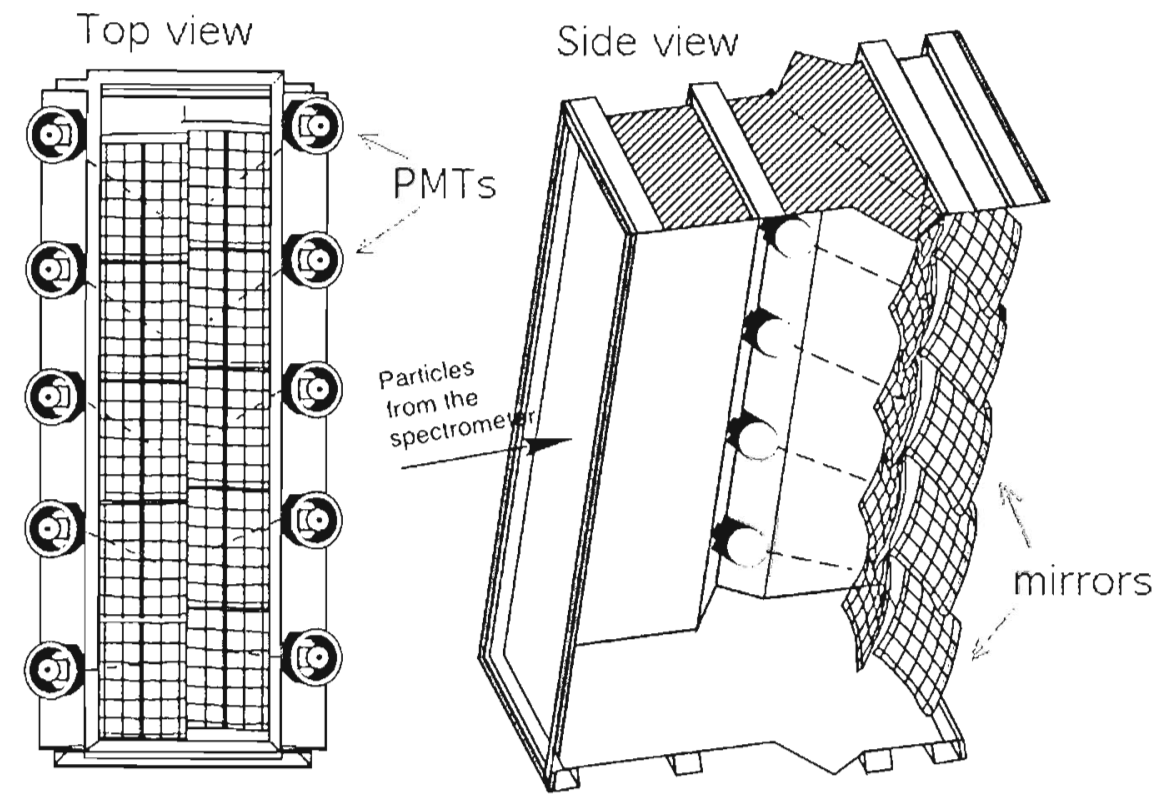

Figure 22: $\mathrm{CO}_{2}$ gas threshold Cherenkov detector, JLab PHY98-06.

The detector is made of steel with thin entrance and exit windows made of Tedlar. It has a 10 spherical mirror arranged as shown in Figure 22 [46]. The mirrors are very light and represent a thickness no more than $0.23 \mathrm{~g} / \mathrm{cm}^{2}$ for the particles passing through. Each mirror is individually coupled to PMTs (Burle 8854), which are located at the focal point of the mirrors $(45 \mathrm{~cm}$ from the mirrors). All the electric signals resulted from the light collected by the PMT, are sent to ADCs. Although pions are not supposed to emit Cherenkov radiation they can still interact with the material, knocking out electrons, that in turn produce Cherenkov radiation. As a result, a small amount of light caused by negative pions will also be observed. However, the light produced as a result of pion interactions will produce fewer numbers of photoelectrons on average compared to those produced by electrons. Consequently, electrons can be distinguished from negative pions. 
The average number of photoelectrons produced by a $1.56 \mathrm{GeV}$ electron in the $\mathrm{CO}_{2}$ gas threshold Cherenkov detector is of $\sim 14$ p.e. [47].

\subsubsection{Lead glass preshower and shower counters}

There are two lead glass counters in the electron arm (HRS-R). The preshower counter consists of an array of $2 \times 24=48$ blocks of lead glass transversely oriented with respect to the directions of the scattered electrons (Figure 23). The shower counter consists of $5 \times 20=100$ blocks of lead glass oriented parallel to the direction of the scattered electrons [32].

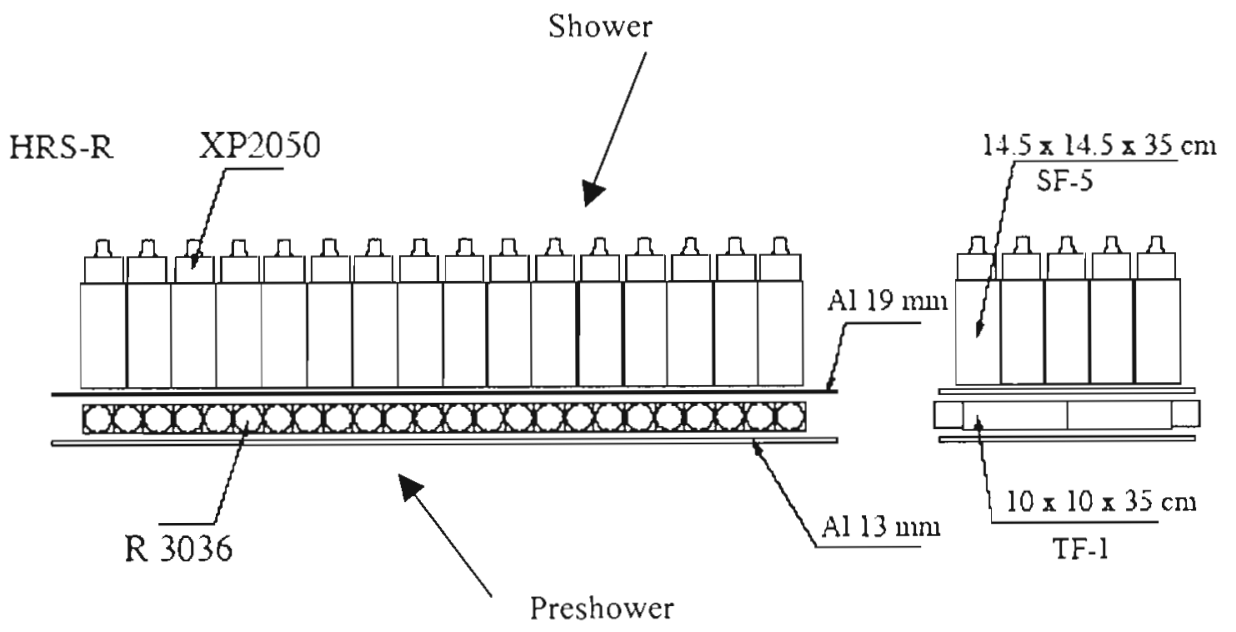

Figure 23: HRS_R shower and preshower counters [32].

Each lead glass block in the preshower is $10 \mathrm{~cm} \times 10 \mathrm{~cm} \times 35 \mathrm{~cm}$ and in the shower is

$14.5 \mathrm{~cm} \times 14.5 \mathrm{~cm} \times 35 \mathrm{~cm}$. They are made of DF6 lead glass with a density of $5.18 \mathrm{~g} / \mathrm{cm}^{3}$. 
These detectors provide additional PID and are located behind the second scintillator plane S2. The signal detected by the lead glass counters is linearly proportional to the energy deposited by the incoming particle. The longitudinal distribution of the energy deposited in the counter can be used to identify the incident particles because, due to the longer hadronic mean free path, hadronic showers do not develop while electromagnetic showers do. Hadrons produce a low ADC signal while electrons produce a high ADC signal. The resolution of the detector comes from separating the tails of the two ADC signals. To improve this resolution a double layer of lead glass counters is used, because electrons will deposit most of their energy in the first layer while hadrons will deposit more of their energy in the second. The proper combination of the two lead glass layers enhances the detection of electrons or pions.

\subsubsection{Aerogel threshold Cherenkov counters}

The aerogel threshold Cherenkov counters are located in the hadron arm (HRS-L) inside the detector stack. There are two aerogel threshold Cherenkov counter detectors called A1 and A2. The purpose of using A1 and A2 in experiment E94-107 is to discriminate kaons from pions and protons. The detectors A1 and A2 form the basic hadron PID procedure, which is further enhanced with a Ring Imaging Cherenkov detector described in Chapter 2.6.6. The A1 and A2 have similar designs. Both are diffusion box types of Cherenkov threshold detectors with different threshold momenta for the different particles. 
The A1 has 24 PMTs Burle RCA 8854 with high quantum efficiencies in UV and visible ranges of the electromagnetic spectrum (see Figure 24). The PMTs are aligned at both sides of the diffusion box, which is internally covered with millipore paper with high reflectivity (more than 95\%). A $9 \mathrm{~cm}$ thickness layer of silica aerogel over a honeycomb structure resides at the bottom and constitutes the Cherenkov radiator. The refractive index of the silica aerogel in $\mathrm{A} 1$ is $\mathrm{n}_{1}=1.015$.

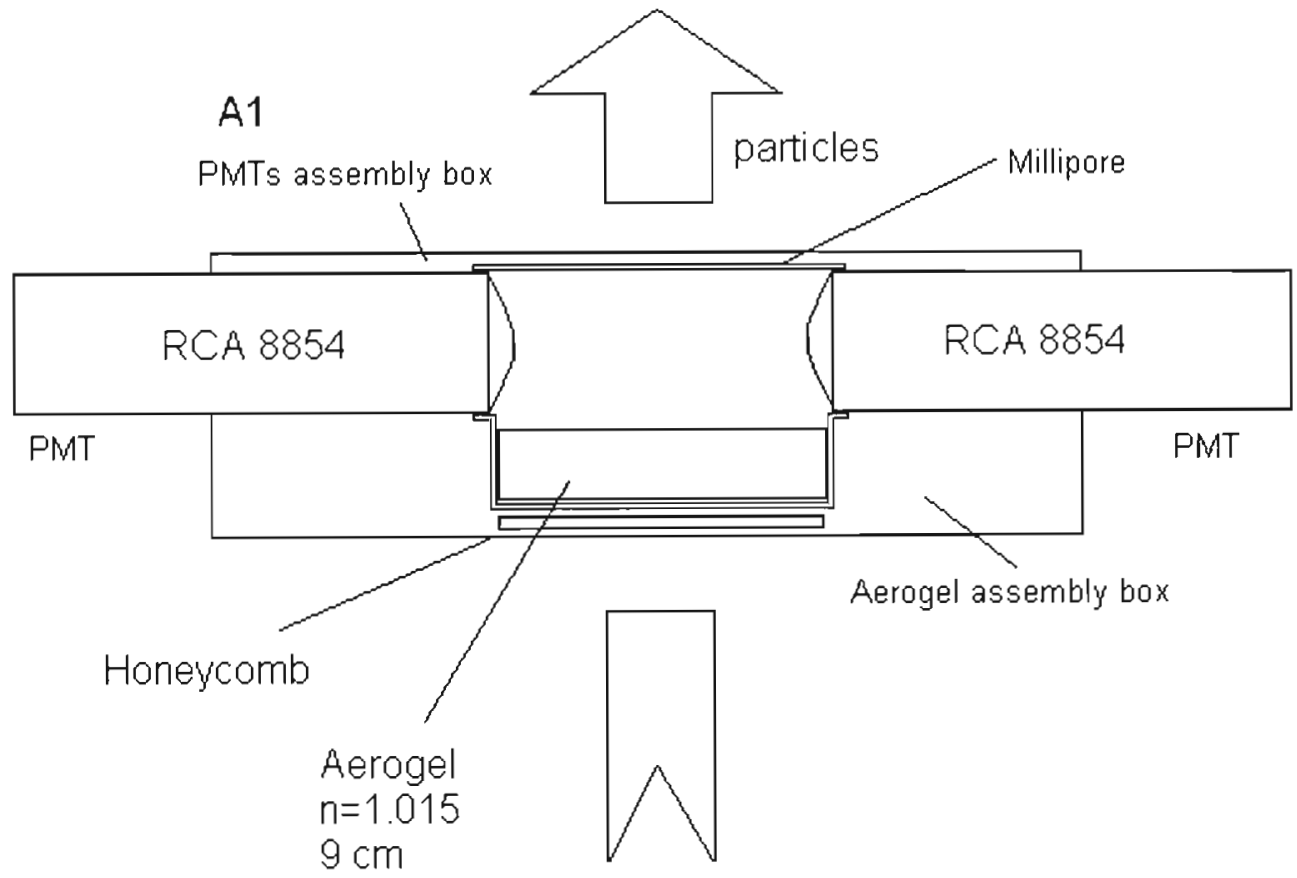

Figure 24: Al silica aerogel Cherenkov detector.

The A2 detector was designed with similar characteristics (see Figure 25), but it has 26 Photonics XP 4572 PMTs and $5 \mathrm{~cm}$ of silica aerogel with a refractive index of $\mathrm{n}_{2}=$ $1.055[32,48]$. 


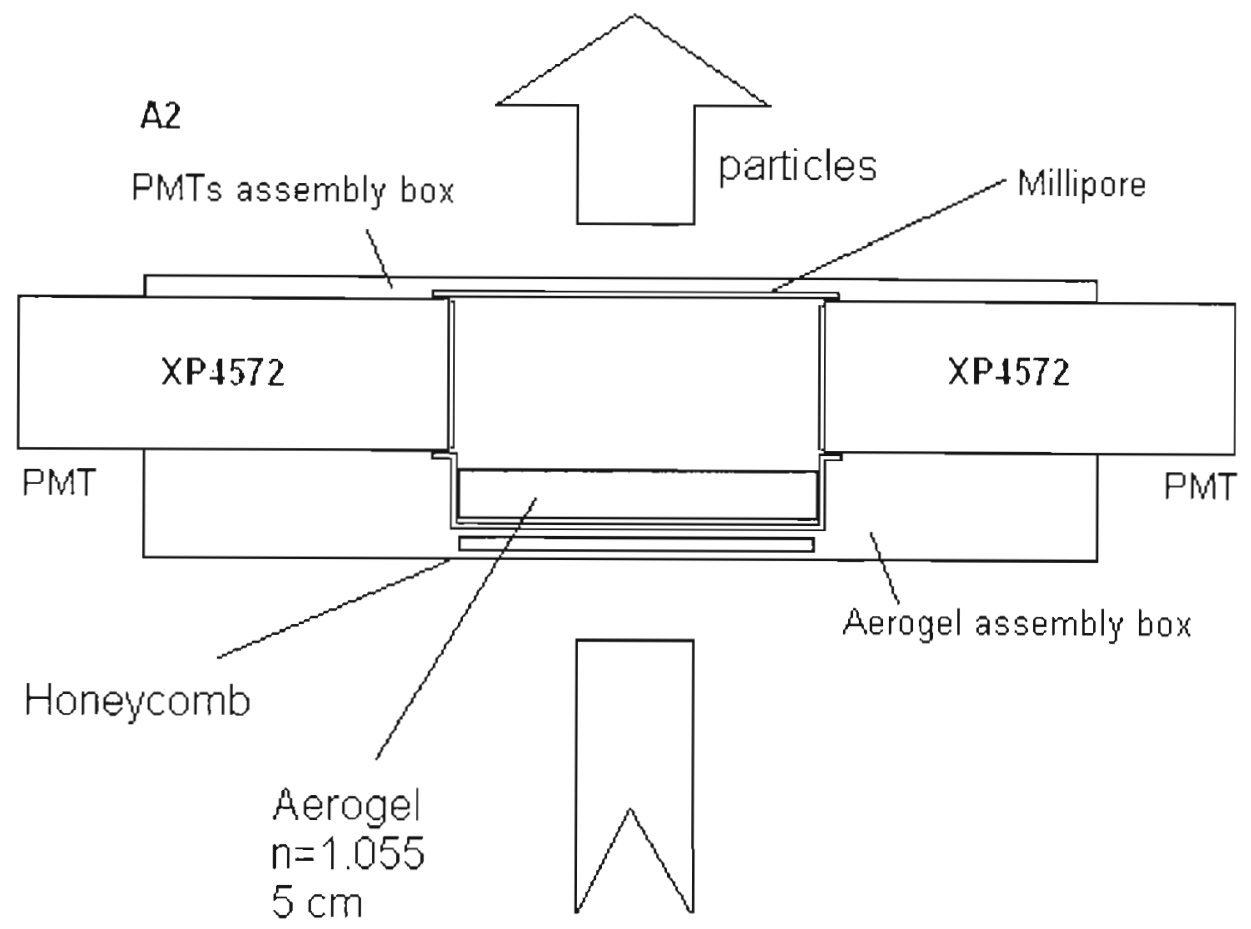

Figure 25: A2 silica aerogel Cherenkov detector.

The PMTs in A2 are different because they do not need to be so sensitive since the number of photoelectrons produced is greater. The greater the refractive index the smaller the threshold momenta and the greater number of photons produced by Cherenkov radiation.

In order to use $\mathrm{A} 1$ and $\mathrm{A} 2$ to discriminate kaons from protons and pions, we took advantage of the different threshold momenta of the particles in the two detectors. Figure 26 shows the threshold momenta for kaons, protons and pions in both detectors [49]. 


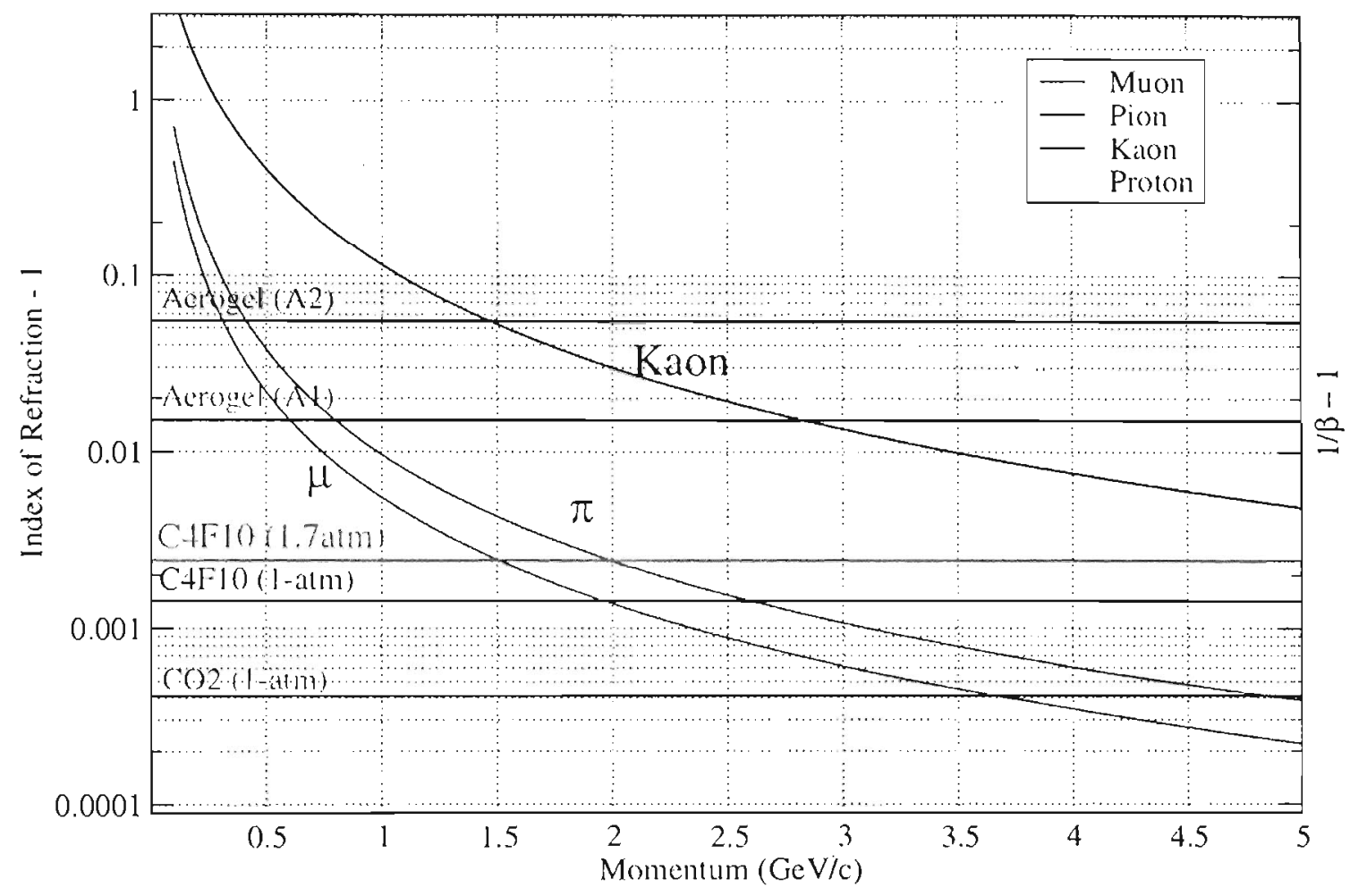

Figure 26: Threshold momenta for different particles in A1 and A2 [48] and private communication with R. Feuerbach.

In experiment E94-107 three different momenta were set in the hadron spectrometer (HRS-L). These momenta were in the range from $1.82 \mathrm{GeV} / \mathrm{c}$ to 1.96 GeV/c. According to the graph shown in Figure 26 for kaons, pions and protons we observe that pions fire both detector $\mathrm{A} 1$ and $\mathrm{A} 2$, protons fire neither $\mathrm{A} 1$ nor $\mathrm{A} 2$, and kaons fire A2 but not A1. 


\begin{tabular}{cc}
\hline Particle & Condition for identification \\
\hline Pions $\left(\pi^{+}\right)$ & A1 \& A2 \\
Kaons $\left(K^{+}\right)$ & $\overline{\mathrm{A} 1} \& \mathrm{~A} 2$ \\
Protons $\left(p^{+}\right)$ & $\overline{\mathrm{A} 1} \& \overline{\mathrm{A} 2}$ \\
\hline
\end{tabular}

Table 8: PID conditions for different particles in A1 and A2.

Specific details and results of the PID procedure are shown in Section 4.1. The average number of photoelectrons (p.e.) obtained per events was of up to 8 p.e. in A1 and of up to 30 p.e. in $\mathrm{A} 2$.

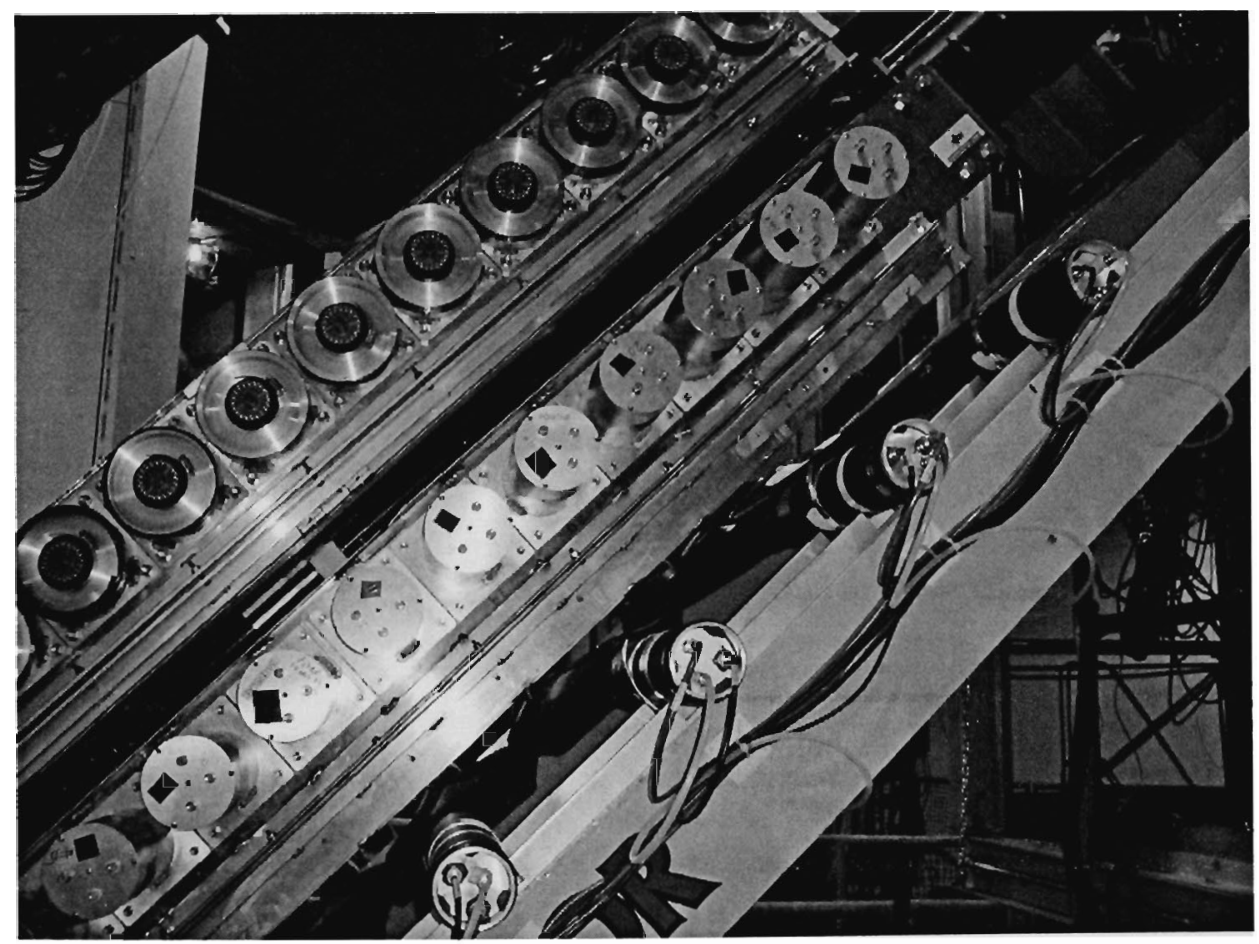

Figure 27: Picture showing from left to right; A2, Al and S1, in the hadron arm. Particles traverse the stack from lower right to upper left. 
2.6.6 Ring imaging Cherenkov detector $(\mathrm{RICH})$

The RICH detector uses the geometrical features of the distribution of the Cherenkov radiation as a tool to identify particles. Basically, Cherenkov radiation is emitted by charged particles moving faster than the speed of light in the medium. The geometrical distribution can be described by $\cos \theta_{c}=1 / \beta n$. This angle is shown in Figure 28.

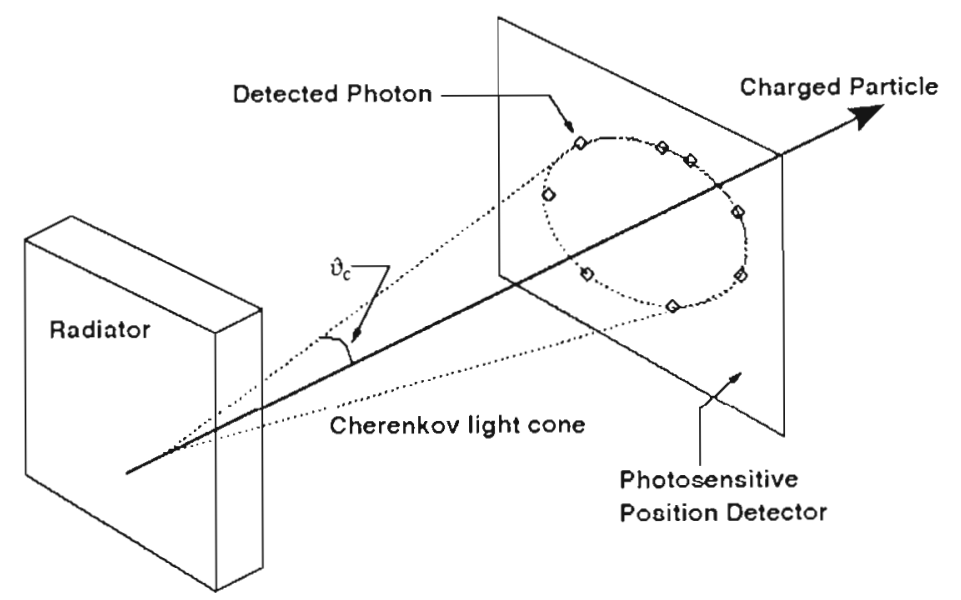

Figure 28: Cherenkov radiation geometrical distribution [50].

Therefore, at different speeds the opening angle of the Cherenkov cone will be different. In the case of experiment E94-107, pions, kaons and protons have different speeds. Their Cherenkov radiation will exhibit different cone angles $\theta_{c}$. 
The Hall A RICH detector has a Neoceram container with Freon $\left(\mathrm{C}_{6} \mathrm{~F}_{14}\right)$ radiator, which has a high refractive index of 1.28 . The exit window of the Neoceram container is made of fused quartz. This design enhances well-defined light cones of focusing Cherenkov radiation with cone angles $\theta_{c}$ as different as possible for the three different particles. The Cherenkov radiation goes through an environment of dry methane $\left(\mathrm{CH}_{4}\right)$. The RICH detector detects the Cherenkov light on a photosensitive plane made of three pads of CsI, and reconstructs the Cherenkov angle of light emitted by the incident particle [51]. After that, the information of the detection of the ring as well as the middle point of ionization are translated into electronic signals. Figure 29 shows schematically the details of the components of the RICH. Figure 30 shows a picture of the RICH at the detector stack.

Pions are expected to be faster than kaons, and kaons to be faster than protons. Therefore, the detected rings produced by pions will have bigger radii than the ones produced by kaons and the detected rings produced by kaons will have bigger radii than the ones produced by protons.

The RICH detector significantly increased the signal to noise ratio. The enhancement in the PID was very important in E94-107 for targets like carbon, beryllium and oxygen. In the case of hydrogen the time of flight provided by the scintillators and the aerogel detectors were enough to have a good PID without losing statistics. In the case of hydrogen, the RICH detector can be used to easily determined the aerogel detector efficiencies as will be described in Section 4.4. 


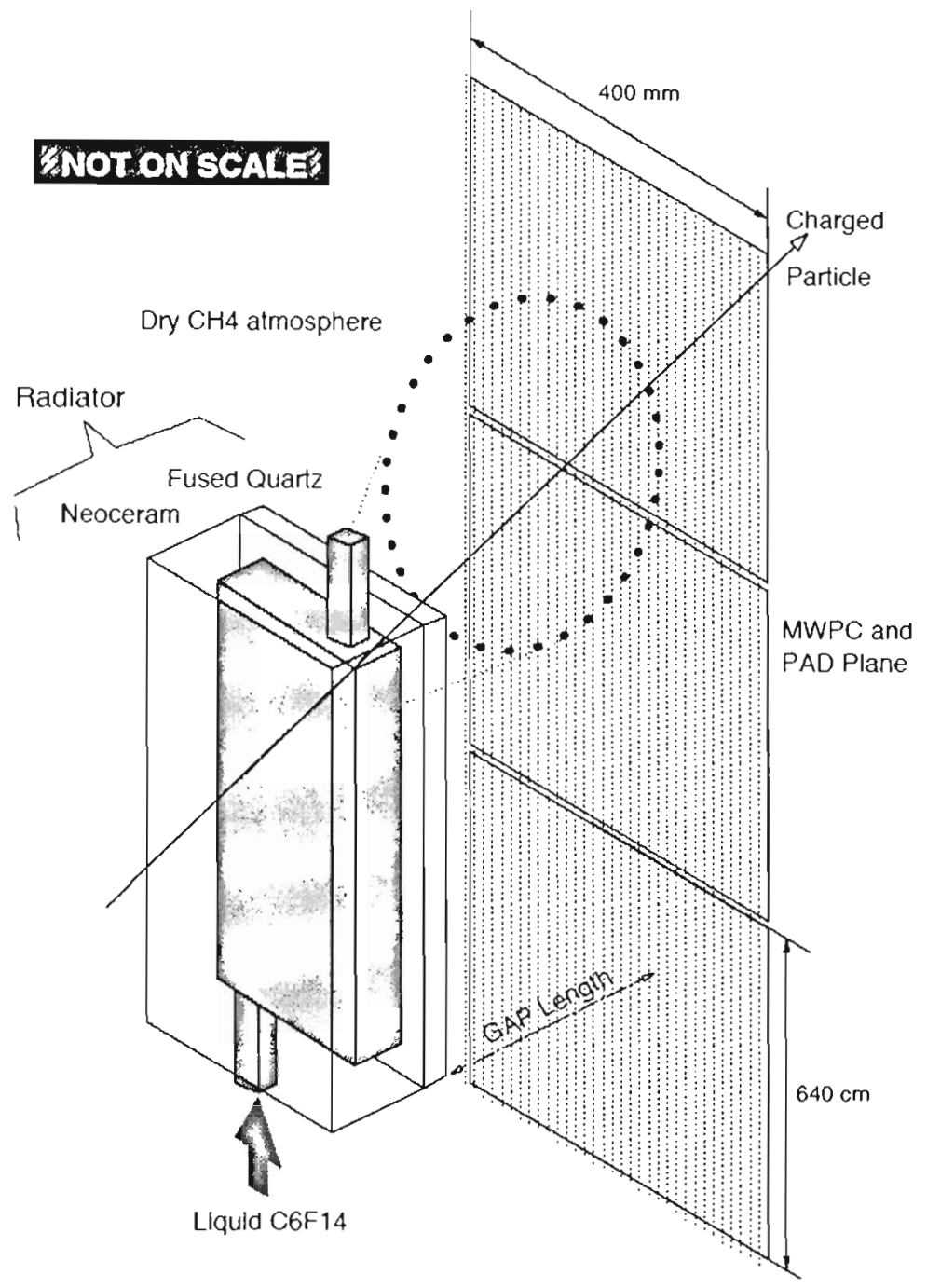

Figure 29: Schematic view of the Hall A operation [50, 51]. 


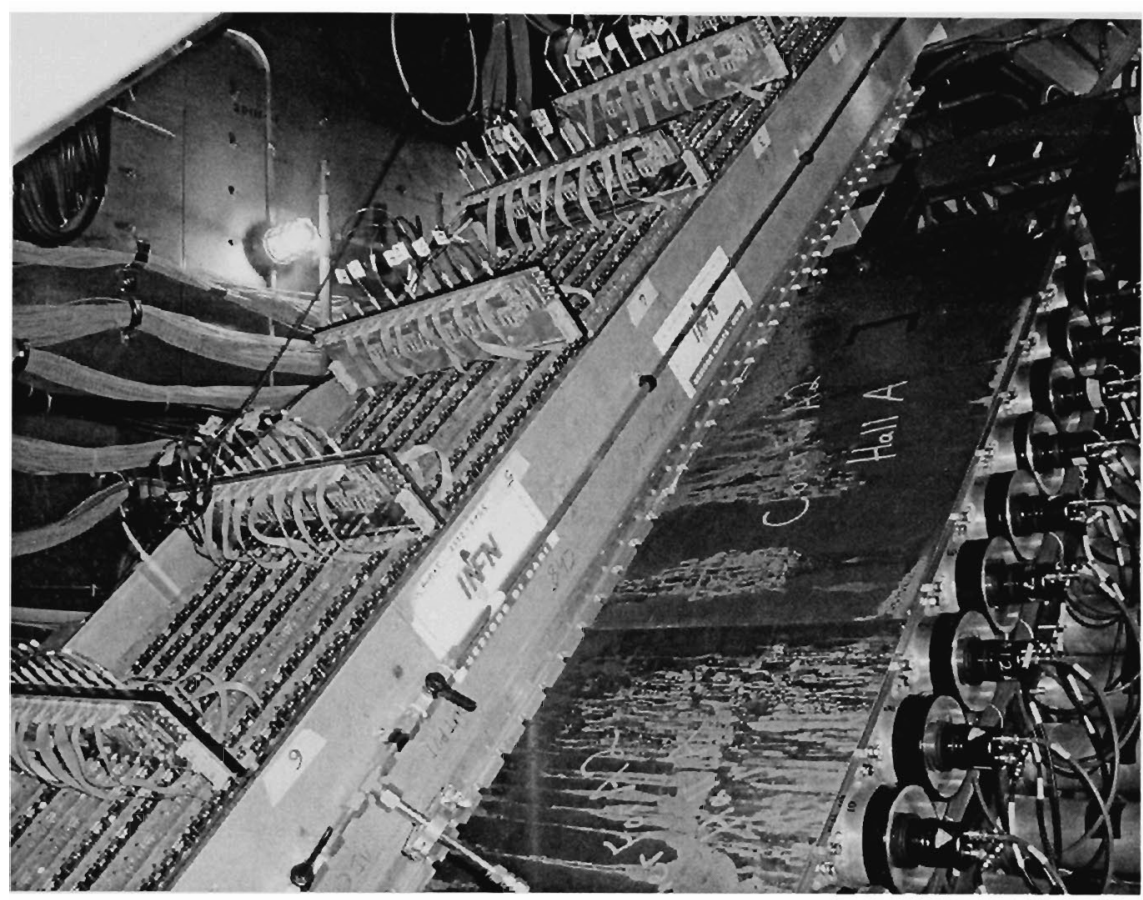

Figure 30: Picture of the RICH detector in the HRS-L detector stack, with the A2 just below it.

\subsection{Data acquisition}

\subsubsection{Data acquisition system}

All the data obtained when running the experiment comes as electronic signals, which have to be properly combined, recorded and monitored to ensure the data quality. The CEBAF On-line Data Acquisition system (CODA) is a special software developed by Jefferson Lab to readout nuclear physics experiments. In order to readout the data coming from the experiment, the acquisition system uses hardware Read Out Controllers (ROC's), an event builder (EB), and an online analyzer. The information about an event, 
such as the run number, information from the detectors, spectrometer magnets settings, angles of scattered particles, scalers data, target temperature and pressure, spectrometers positions, and beam; energy, current and profile, are transferred to the recording computers by the ROC's. After that, the information is combined and organized by the EB and then is incorporated into a common event format [52]. Then the online analyzer system is used to analyze the data and save it to the hard drives, and later, transferred to storage devices such as tapes. The online analyzer is a very important tool since it allows us to take a preliminary view of the output of the experiment to ensure that the detectors and electronic equipment are working properly while acquiring the data.

A single Run Control process controls a network of computers used to run the CODA acquisition system. The Run Control function runs the ROC subsystems, the EB, the EA, and the ER. The ADCs, TDCs and scalers in FASTBUS and VME crates monitor and process signals coming from the detectors. Three independent ROC's, each of them reading/recording part of the information associated with an event, allow the system to have over 20 front-end crates (FASTBUS and VME), with a FASTBUS interface that can read out up to 10000 detector channels.

Finally, the EB receives the data from the ROC's and checks for any missing data. Then, the EB writes the data for each event onto disk. 


\subsubsection{Trigger electronics}

The primary trigger signals come from the scintillators' PMT anode signals. The signals from the detectors are sent to the ADCs and to a discriminator. If the discriminator senses a signal greater than its threshold, whose leading edge lies within a narrow time window, then it sets an output. The VME settable registers control the time window width and offset.

The discriminator time-over-threshold signals are compared to signals coming from other scintillator detectors. In the case of coincidence experiments, a coincidence is formed between the spectrometer arms. The main trigger is formed when both scintillator planes S1 and S2 fire. Therefore, there must be a hit in both PMTs of one paddle in S1 and one in S2. The coincidence between spectrometers is an overlap AND circuit. The right spectrometer single triggers are called $\mathrm{T} 1$ and the ones in the left spectrometer are called T3, while the coincidence triggers are called T5. The aerogel Cherenkov detectors produced a trigger, $\mathrm{T} 6$, when a kaon was detected. $\mathrm{T} 7$ is created by having $\mathrm{T} 5$ and $\mathrm{T} 6$ in coincidence (coincident kaons). The main configuration of the triggers is in Table 9:

\begin{tabular}{ll}
\hline & \multicolumn{1}{c}{ TRIGGERS } \\
\hline T1 & Electron or right HRS (T1) (S1 and S2) \\
T2 & Electron or right HRS (T2) \\
T3 & Hadron or left HRS (T1) (S1 and S2) \\
T4 & Hadron or left HRS (T2) \\
T5 & HRS R \& HRS L coincidence \\
T6 & Aerogel \\
T7 & Aerogel and coincidence \\
T8 & Pulser \\
\hline
\end{tabular}

Table 9: Main configuration of triggers in E94-107. 
Additional T2 and T4 triggers are formed when only 3 PMTs fire in the right arm and in the left arm respectely, and they constitute looser triggers. The T2 and T4 triggers are useful for the Scintillators' efficiencies analysis described in Section 3.4.

The delays for the trigger system in Hall A can be configured remotely through CAMAC modules. Sometimes during an experiment the delays need to be adjusted for the timing of triggers that change with particle momenta and particle identification (PID). However, these delays are relevant for coincidence setups only. The signals from the detectors are sent to the Memory Logic Units (MLUs). The output of the MLUs for the experiment is ORs and ANDs of all detectors for which they fired. The MLUs in each spectrometer arm generated a logical output (S-ray) when both PMTs of a scintillator paddle in $\mathrm{S} 1$ and in $\mathrm{S} 2$ of an adjacent or coincident paddle fired. 
Single Arm Triggers in Each Spectrometer

R. Michaels (Aug 2003)

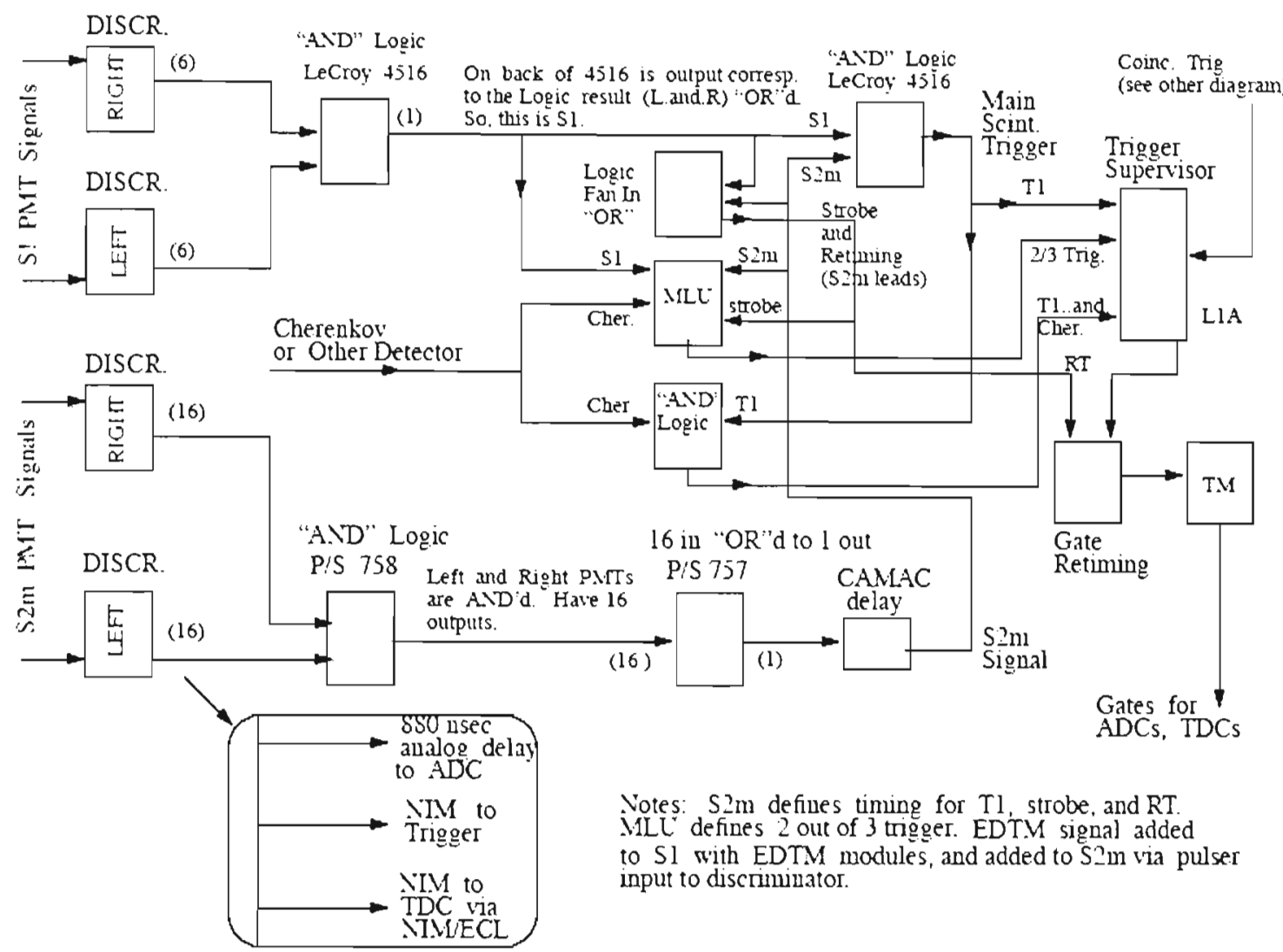

Figure 31: Single arm trigger in electron/hadron spectrometer in Hall A [53].

In Figure 31 a schematic of the single arm triggers in either the electron or hadrom spectrometer is shown. T5 constitutes the coincidence trigger and T7 the kaon coincidence trigger. A T5 is obtained each time a single arm S-ray triggers a logical AND unit. There is a time window setup of approximately $100 \mathrm{~ns}$ for the coincidence to be accepted as such. Furthermore, the number of events for every trigger type is recorded by a running scaler, which is read and logged by the data acquisition system (DAQ) every 10 seconds. Although, all triggers can fire the DAQ, it is $\mathrm{T} 7$ that has the priority and the 
other triggers are prescaled. Therefore, only a fraction of the events from triggers other than T7 are recorded. The fractions are set using prescale factors named PS1, PS2, PS3, PS4, PS5, PS6 and PS7. Consequently, T7=1 and the second priority is for T5 so it has the smallest acceptable PS5 (typically $\sim 3$, see Section 3.4). The encoding of the analog signals and the transfer of the digitized signal to the computer buffers takes about $700 \mu \mathrm{s}$.

The T7 represents the true coincidence events for ${ }^{\mathrm{H}} \mathrm{H}\left(e, e^{\prime} K^{+}\right) \Lambda, \Sigma^{0}$, for that reason it was not prescaled $(\mathrm{PS} 7=1)$. Figure 32 shows schematically the coincidence trigger configuration in Hall A:

Coincidence Trigger

Electronics on Left Arm
R. Michaels (Aug 2003)

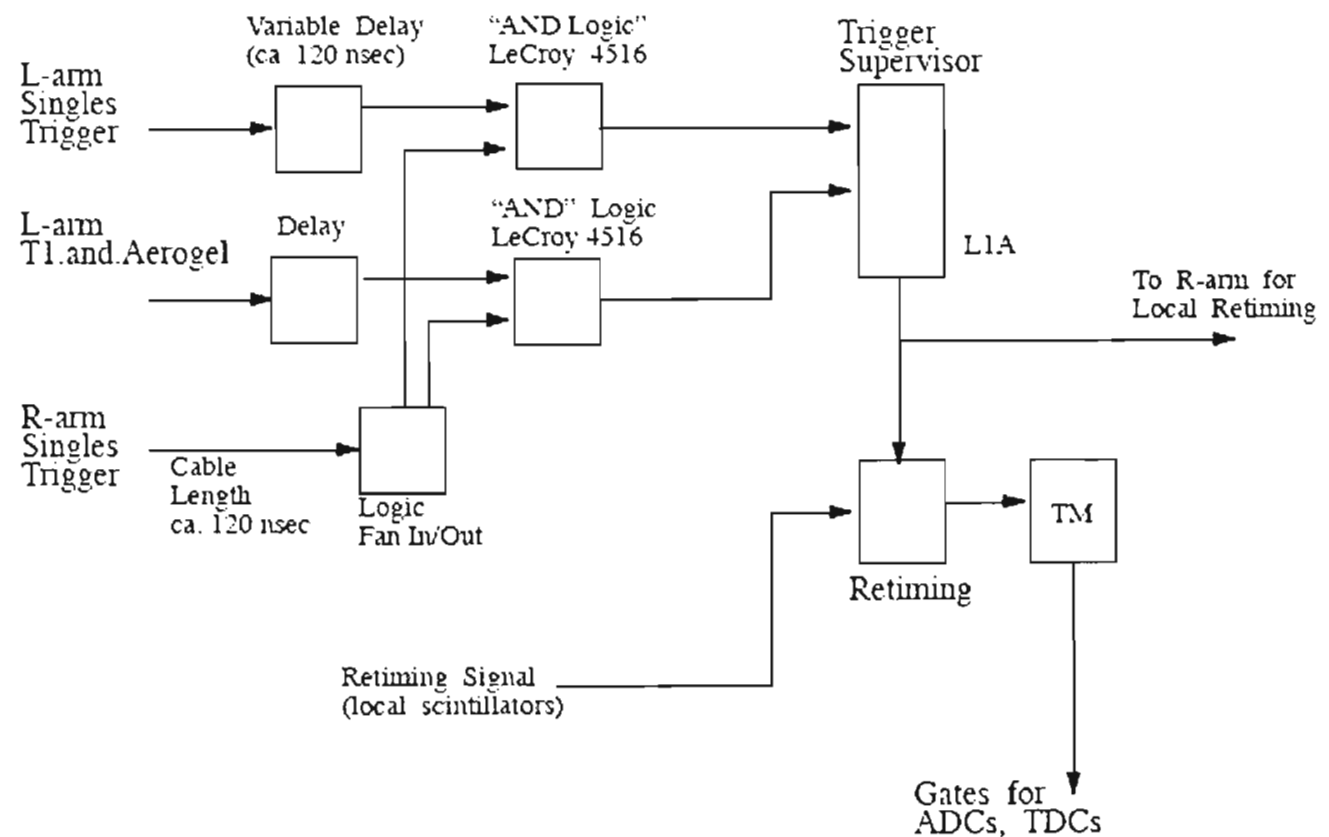

Figure 32: Coincidence trigger configuration in Hall A [53]. 
3 Calibration, corrections and efficiency measurements

A brief description of coordinate systems, optics calibration and some of the corrections and efficiency measurements without PID software analysis are presented in this section, which are important pieces of information that later, will be used in Section 4 to extract the cross section for the reactions ${ }^{1} \mathrm{H}\left(e, e^{\prime} K^{+}\right) \Lambda, \Sigma^{0}$. To obtain this information specific programming codes have been created that not only provide efficiencies but also particle tracking reconstruction.

\subsection{Coordinate systems}

\subsubsection{Hall A coordinate system (HCS)}

Usually, the origin of the HCS is at the center of the hall, as defined by the intersection of the electron unrastered beam and the vertical symmetry axis of the target lifter assembly on the hall's spectrometer pivot. However, as a result of the insertion of the septum magnets the optical center was moved $80 \mathrm{~cm}$ upstream from the its normal position. A top view of the HCS is shown in Figure 33. The $z$ axis is along the beam line and points in the direction of the beam dump, $y$ is vertically upward, and $\hat{x}=\hat{y} \times \hat{z}$ [32]. 


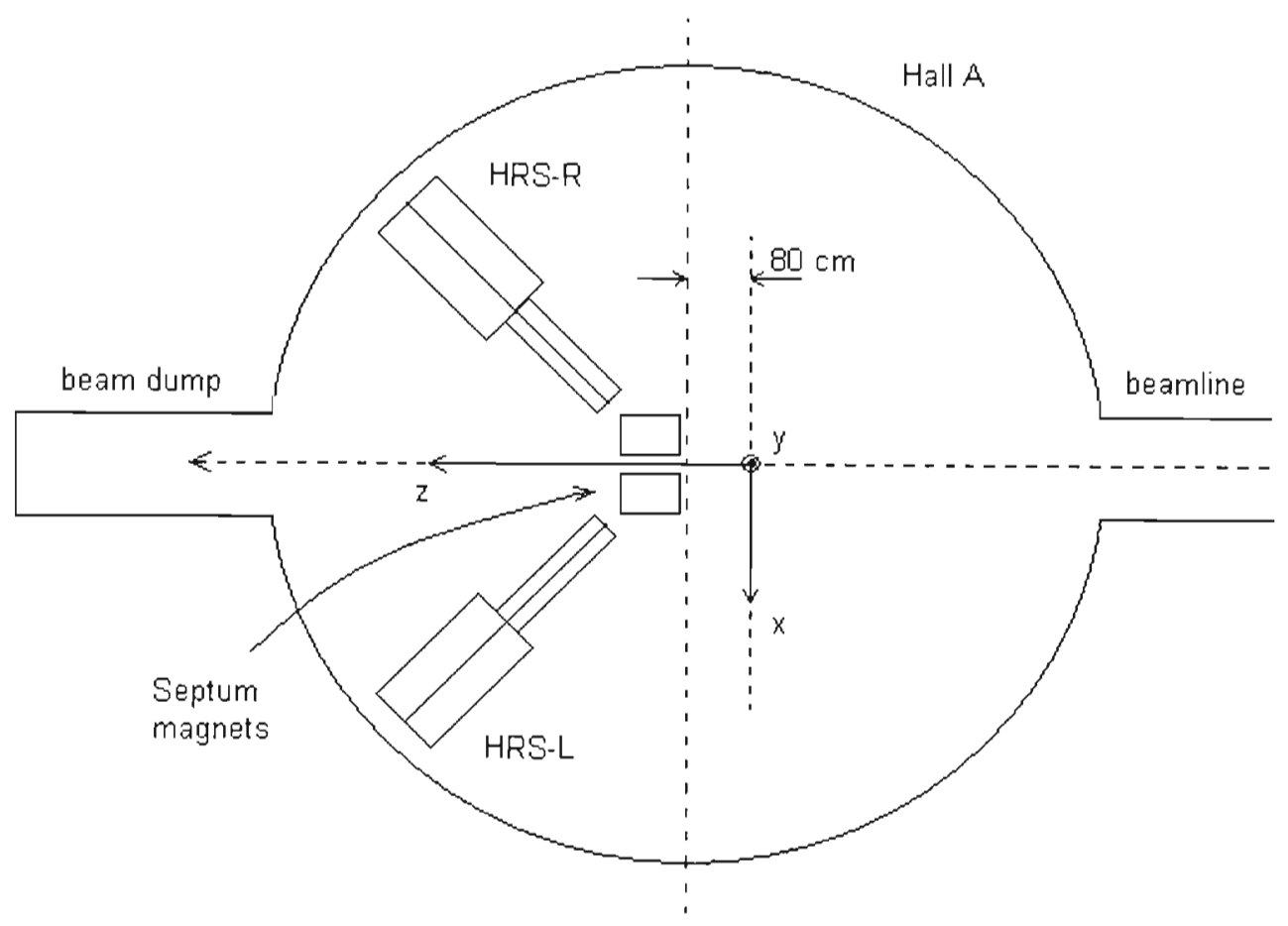

Figure 33: Top view of the Hall A coordinate system.

\subsubsection{Target coordinate system (TCS)}

The TCS is referenced by the line passing through the target along the central momentum of either of the spectrometers. The line defines, at the target, the $z$ axis of the TCS for a given spectrometer. Consequently, each spectrometer has its own TCS. The $x$ axis is the line crossing perpendicularly both, the electron beam line and the $\mathrm{z}$ axis, and it points downward (see Figure 34). The $x z$ plane determines the $y$ axis of the TCS. The triplet $\hat{x}, \hat{y}$ and $\hat{z}$ is right handed. Variables referring to the coordinates at the target are designed by the subscript "tg". Therefore, variables $x_{t g}$ and $y_{t g}$ are defined as the $x$ and $y$ 
coordinates of the point of intersection of a particle trajectory with the $z_{t g}=0$ plane. The variables $\theta_{\lg }$ and $\phi_{\lg }$ are defined as [32]:

$$
\begin{aligned}
& \tan \theta_{t g}=\frac{d x_{t g}}{d z_{t g}} \\
& \tan \phi_{t g}=\frac{d y_{t g}}{d z_{t g}}
\end{aligned}
$$

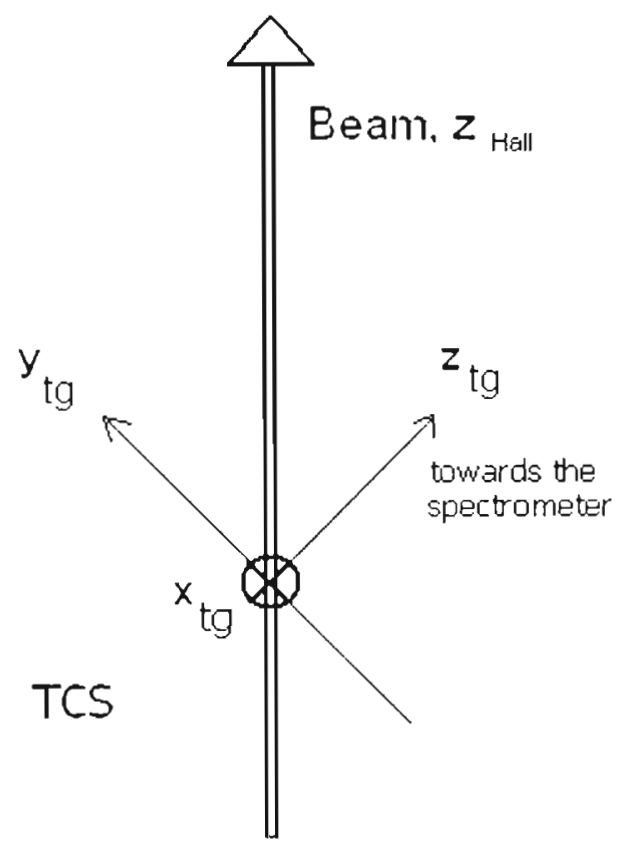

Figure 34: Target coordinate system in Hall A (top view).

The relative momentum $\delta_{t g}$ is defined by $\delta_{t g}=\frac{\left(p-p_{0}\right)}{p_{0}}$, where $p$ is the particle momentum and $p_{0}$ is the spectrometer central momentum. 


\subsection{Optics calibration}

In Hall A, conventionally the two HRS are an identical pair of QQDQ magnetic spectrometers with optical properties that are point-to-point in the dispersive direction. However, for E94-107 septum magnets were added, making each spectrometer arrangement into a Septum + QQDQ, which required a recalibration of the optics. The main purposes of the optics calibration are to improve the accuracy in reconstructing the variables at the target from the variables observed at the VDC detectors (at the focal plane) and to independently establish the spectrometer resolution [54].

The optics database is a set of tensor elements defining the transport tensor which links the coordinates measured at the focal plane of the spectrometers with the angular, spatial coordinates at the target, and the momentum. The focal plane coordinates are described by the four quantities; $x_{f p}, y_{f p}, \theta_{f p}, \phi_{f p}$, A detailed description of the coordinate systems used is given in $[55,56]$. When the left-right symmetry of the spectrometers with respect to their vertical mid-planes holds then the relationship between the focal plane and target coordinates could be written in a first-order approximation as a simple matrix given by:

$$
\left(\begin{array}{l}
\delta \\
\theta \\
y \\
\phi
\end{array}\right)_{t g}=\left(\begin{array}{cccc}
\langle\delta \mid x\rangle & \langle\delta \mid \theta\rangle & 0 & 0 \\
\langle\theta \mid x\rangle & \langle\theta \mid \theta\rangle & 0 & 0 \\
0 & 0 & \langle y \mid y\rangle & \langle y \mid \phi\rangle \\
0 & 0 & \langle\phi \mid y\rangle & \langle\phi \mid \phi\rangle
\end{array}\right)\left(\begin{array}{l}
x \\
\theta \\
y \\
\phi
\end{array}\right)_{f p}
$$

where $x_{t g}$ is determined independently by BPM measurements. 
However, the insertion of the septum magnets destroys the symmetry of the spectrometers with respect to their vertical mid-planes so the matrix (29) gets replaced with one that is more complicated (e.g., has more non-zero elements). Thus, the target variables are given by:

$$
\begin{aligned}
& y_{t g}=\sum_{i j k l} Y_{i j k l} x_{f p}^{i} \tan ^{j}\left(\theta_{f p}\right) y_{f p}^{k} \tan ^{l}\left(\phi_{f p}\right), \\
& \tan \left(\theta_{t g}\right)=\sum_{i j k l} T_{i j k l} x_{f p}^{i} \tan ^{j}\left(\theta_{f p}\right) y_{f p}^{k} \tan ^{l}\left(\phi_{f p}\right), \\
& \tan \left(\phi_{t g}\right)=\sum_{i j k l} P_{i j k l} x_{f p}^{i} \tan ^{j}\left(\theta_{f p}\right) y_{f p}^{k} \tan ^{l}\left(\phi_{f p}\right), \\
& \delta_{t g}=\sum_{i j k l} D_{i j k l} x_{f p}^{i} \tan ^{j}\left(\theta_{f p}\right) y_{f p}^{k} \tan ^{l}\left(\phi_{f p}\right),
\end{aligned}
$$

and the tensor elements; $Y_{i j k l}, T_{i j k l}, P_{i j k l}, D_{i j k l}$ are polynomial expansions on $x_{f p}$ up to the fifth order $(i+j+k+l \leq 5)[57]$.

The database optimization starts with sets of data from elastic scattering on a thin ${ }^{12} \mathrm{C}$ target. Generally, 5 open collimator measurements are performed at $\Delta p / p$ values stepping between $-4.5 \%$ and $+4.5 \%$ of the spectrometer central momentum, so that the elastic peak moves across the focal plane. The sieve slit is used to optimize the reconstruction of the angular coordinates. It is positioned behind the target at the entrance of the septum magnets. Each slit has 49 holes of different sizes in well-defined $x_{\text {sieve }}$ and $y_{\text {sieve }}$ positions as shown in Figure 35 . 


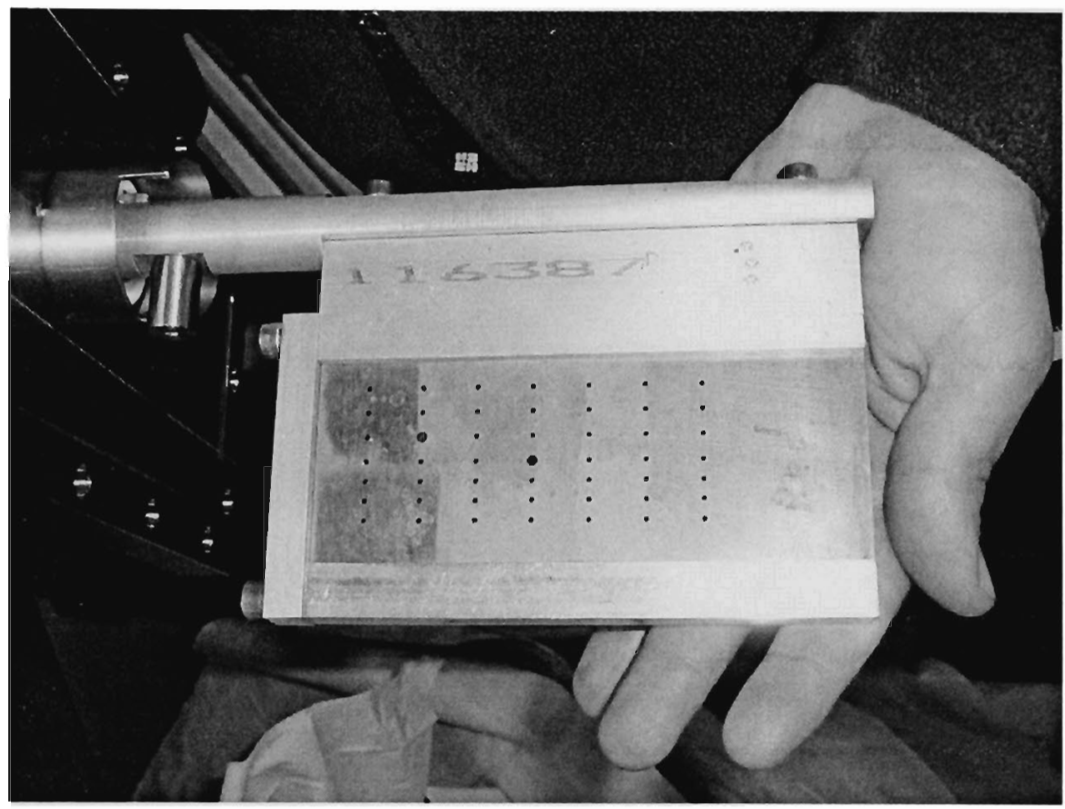

Figure 35: Picture of the sieve used in Hall A.

An iterative procedure is performed in analysis to obtain the best tensor elements for the optics database. Optics tensor elements for both spectrometers have been optimized over the full ranges many times prior to the insertion of the septum magnets making the process well understood. However the E94-107 setup is the first optimized setup with both septum magnets included. Furthermore, the $\mathrm{LH}_{2}$ cryotarget used in E94107 was only $4 \mathrm{~cm}$ long. The modest length target together with the kinematics of E94107 (which was carried out at very forward angles), made the target look quite thin from the point of view of the magnetic spectrometers. Thus, the iterative process of optimization converged faster to the optimal values for the tensor elements

Worthy of note, the spectrometer optics is very sensitive to the values of the magnetic fields in Q2 and Q3. Since the quadrupoles are set by current and not by field, a cycling procedure is required because of the hysteresis effects in the magnets [58]. Figure 36 shows the angular reconstruction of the sieve slit plotting the axes in millimeters. 

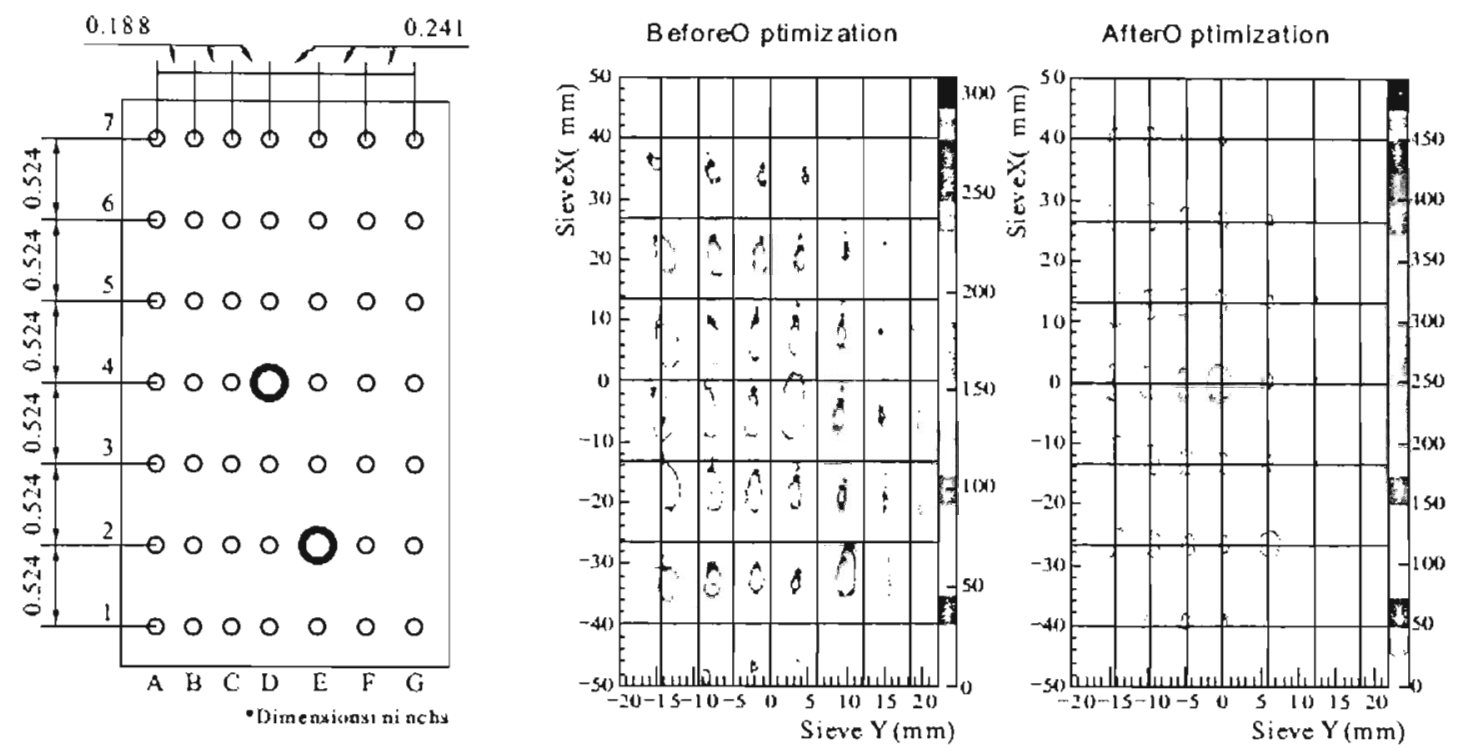

Figure 36: Sieve slit design (left), sieve reconstruction, before optimization (central) and after optimization (right) (Dec 2003) [57].

The optics optimization analysis was performed at the same spectrometer settings as the experiment. The result of the $\delta$ optimization for the kinematics settings of E94-107 corresponded to an average momentum resolution of $\delta p / p=1.8 \times 10^{-4} \mathrm{FWHM}$ on the left arm and $\delta p / p=1.6 \times 10^{-4} \mathrm{FWHM}$ on the right arm. By selecting the central hole of the sieve slit, a slightly improved resolution of $1.4 \times 10^{-4}$ was obtained on both HRS arms as was expected for the HRS [32]. The elastic scattering peak width includes the incident beam energy spread, yielding a measured sigma of $1.2 \times 10^{-4}$ (not FWHM, see Figure 37). 


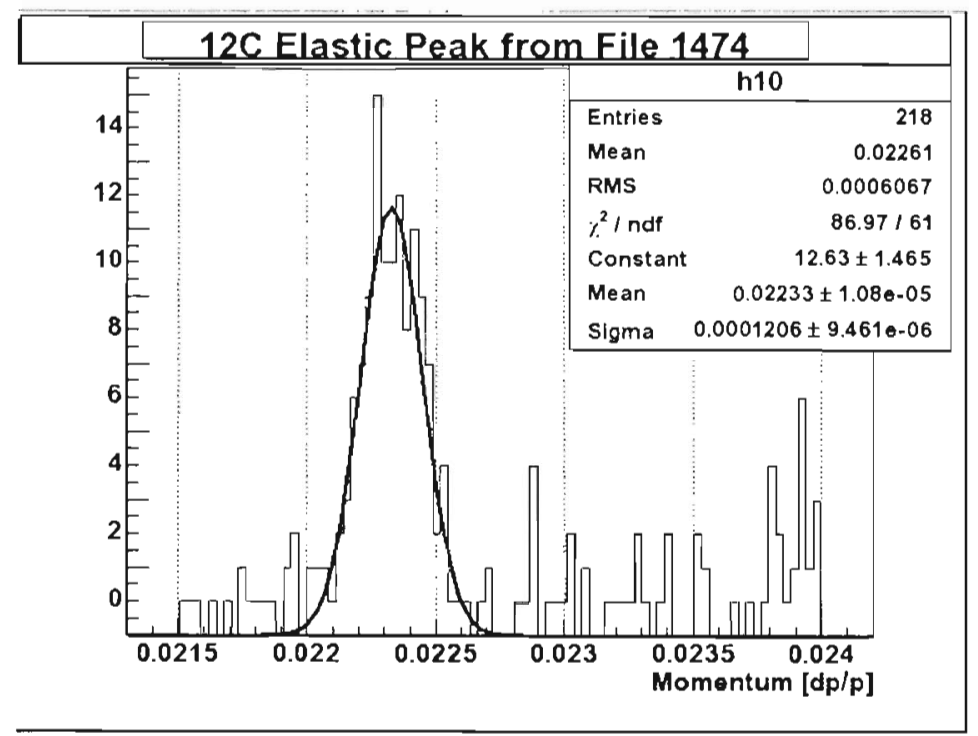

Figure 37: Hall A momentum resolution with septum magnets after optics optimization on a thin ${ }^{12} \mathrm{C}$ target (Hall A electronic logbook, December 2003).

\subsection{Kaon absorption}

Particles traverse different materials along the path from the target up to the detector stack. Consequently, some kaons are absorbed [59]. Dr. Joerg Reinhold developed a program using the kaon-proton cross-section information in the Particle Data Book [60] and on the basis of the eikonal approximation [61] to calculate the kaon absorption depending on the kaon momentum [62]. The program was written in Fortran and it basically determines the ratio of the real to the imaginary part of the kaon nucleon scattering amplitude for forward angles by fitting the $K^{+}$absorption data on ${ }^{12} \mathrm{C}$ from the Particle Data Book [60]. The kaon absorption is proportional to this ratio. 
In order to run the program it needed to be adapted to the conditions of experiment E94-107. The program requires detailed information on the thickness of the different materials the kaons traverse on the way to the final detector, including the density and the atomic weight of these materials.

Figure 38 shows how the absorption of kaons decreases as momentum increases. These results show a slowly varying uncertainty, which is sensible considering the small momentum acceptance of $\pm 4 \%(\delta p / p)$ in the magnetic spectrometer. The program containing the list of all materials is in Appendix A.

During experiment E94-107 three slightly different momenta were set in the hadron arm (HRS-L) to allow some acceptance studies as will be shown in Section 4. Therefore, the kaon absorption coefficients and the associated uncertainties were calculated for those three momenta as shown in Table 10:

\begin{tabular}{lccccccc}
\hline & \multicolumn{5}{c}{$\begin{array}{c}\text { Variation in } \\
\text { momentum }\end{array}$} & \multicolumn{4}{c}{$\begin{array}{c}\text { Variation in } \\
\text { absorption }\end{array}$} \\
\hline KIN & $\begin{array}{c}\text { Kaon } \\
\text { Momentum } \\
(\mathrm{GeV} / \mathrm{c})\end{array}$ & $-4 \%$ & $4 \%$ & absorption & $-4 \%$ & $4 \%$ & uncertainty \\
& & & & & & & \\
KIN_1 & 1.8665 & 1.7918 & 1.9412 & 0.03585 & 0.03736 & 0.03450 & 0.00143 \\
KIN_2 & 1.8218 & 1.7489 & 1.8947 & 0.03672 & 0.03821 & 0.03541 & 0.00140 \\
KIN_3 & 1.9600 & 1.8816 & 2.0384 & 0.03415 & 0.03559 & 0.03283 & 0.00138 \\
\hline
\end{tabular}

Table 10: Kaon absorption and uncertainties in E94-107. 


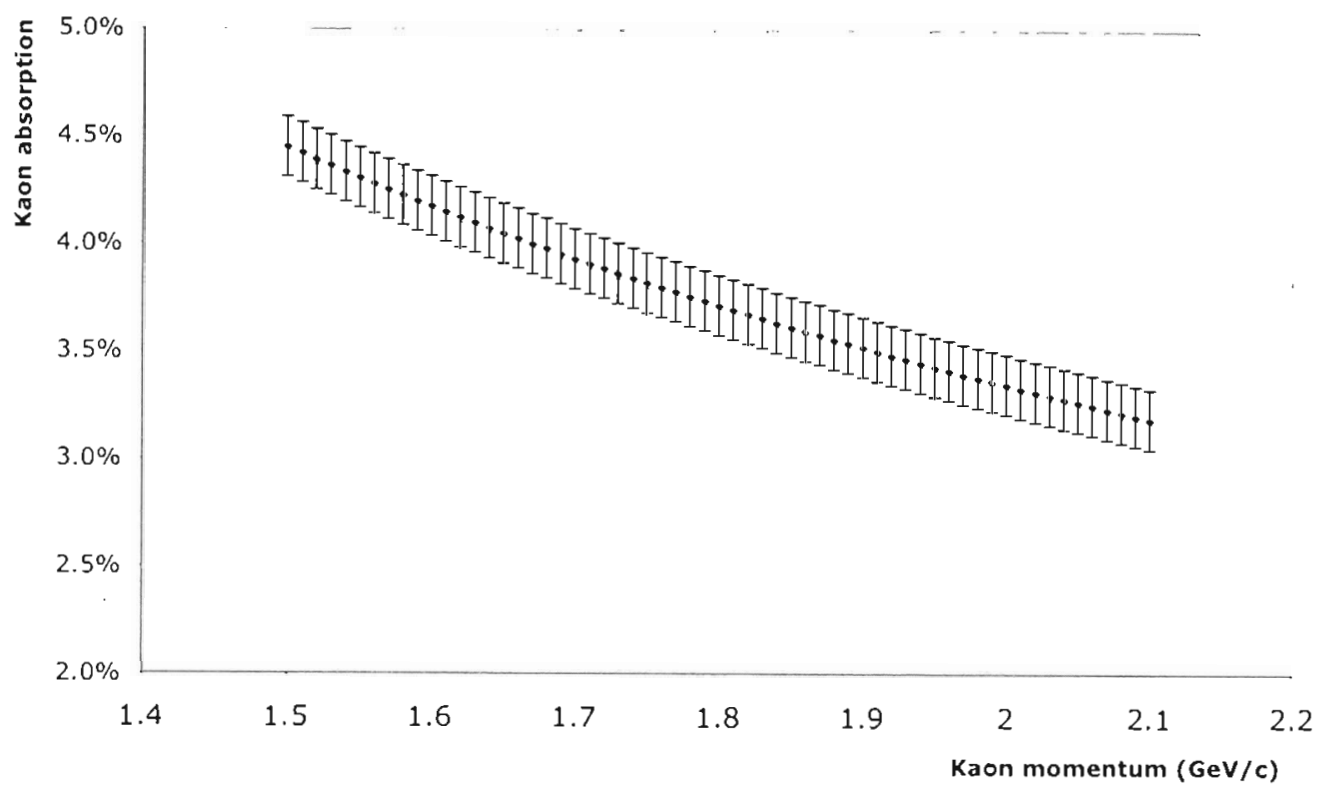

Figure 38: Kaon absorption vs. kaon momentum in E94-107. The errors reflect the $\pm 4 \%$ variations on $\delta p / p$.

\subsection{Scintillators' efficiencies}

The trigger types must be considered carefully to determine the scintillator efficiencies. The basic triggers are T1 (electron arm) and T3 (hadron arm). They are formed by the overlap of the S1 and S2 scintillator planes. The description of the scintillators is in Section 2.6.1. The triggers $\mathrm{Tl}$ and $\mathrm{T} 3$ are formed when the 4 photomultipliers (PMTs) of the two overlapping paddles of scintillators fire in their respective spectrometers. The less stringent $\mathrm{T} 2$ (electron arm) and T4 (hadron arm) are formed when only 3 PMTs fire instead. In this sense T2 and T4 are looser triggers than 
$\mathrm{T} 1$ and $\mathrm{T} 3$. The $\mathrm{T} 5$ triggers are formed by the $\mathrm{T} 1$ and $\mathrm{T} 3$ in coincidence. In addition, there is also T6 trigger formed by the aerogel detectors (see Section 2.6.5) when there is a positive identification of a kaon, and finally there is $\mathrm{T} 7$ trigger that is formed by having T6 and T5 triggers, in other words kaons in coincidence.

Imposing a pre-scale factor to a certain trigger determines the number of events that will be rejected in between storing the event with that particular trigger type. The pre-scale factors are labeled as PS1, PS2, ... to PS7. Consequently, the trigger T7 that is formed by the most interesting events has a PS7 $=1$. Nevertheless, all T5, T6 and T7 are analyzed to maximize our statistics. As a consequence, other particles like protons and pions will be also counted and particle identification cuts are needed to discriminate kaons from protons and pions (see Section 4.1). In order to obtain the scintillators' efficiencies the ratio of all events that fired 4 PMTs to all events that fired at least 3 PMTs is considered. Therefore, on the basis of the previous lines the following equations are used to evaluate the scintillator efficiencies in the electron arm $\left(E_{e}\right)$ and in the hadron $\operatorname{arm}\left(\mathrm{E}_{\mathrm{H}}\right)$ :

$$
\begin{aligned}
& \mathrm{E}_{\mathrm{e}}=\frac{\mathrm{PS} 1 \cdot \mathrm{T} 1+\mathrm{PS} 5 \cdot \mathrm{T} 5+\mathrm{PS} 7 \cdot \mathrm{T} 7}{\mathrm{PS} 1 \cdot \mathrm{T} 1+\mathrm{PS} 2 \cdot \mathrm{T} 2+\mathrm{PS} 5 \cdot \mathrm{T} 5+\mathrm{PS} 7 \cdot \mathrm{T} 7} \\
& \mathrm{E}_{\mathrm{H}}=\frac{\mathrm{PS} 3 \cdot \mathrm{T} 3+\mathrm{PS} 5 \cdot \mathrm{T} 5+\mathrm{PS} 6 \cdot \mathrm{T} 6+\mathrm{PS} 7 \cdot \mathrm{T} 7}{\mathrm{PS} 3 \cdot \mathrm{T} 3+\mathrm{PS} 4 \cdot \mathrm{T} 4+\mathrm{PS} 5 \cdot \mathrm{T} 5+\mathrm{PS} 6 \cdot \mathrm{T} 6+\mathrm{PS} 7 \cdot \mathrm{T} 7}
\end{aligned}
$$

The total efficiency then can be measured just by multiplying the Equations (34) and (35) [63]. 
$\mathrm{E}_{\text {Scint }}=\mathrm{E}_{\mathrm{e}} \times \mathrm{E}_{\mathrm{H}}$

It is also important that a "good" sample of events is being used when measuring the efficiencies. To obtain good events, geometrical cuts are used on the active area of the scintillators (to avoid the edges) and a projected particle track can be required to pass through that active area. Although, this ensures a better determination of the scintillators' efficiencies there is a negative effect, which is to decrease in statistics. Usually in doing analysis physicists try to optimize these competing requirements. However, in E94-107 with the hydrogen cryotarget the number of electron-kaon coincidence events was fairly small so instead a larger number of good events was obtained by not imposing particle ID cuts on the detectors (see Section 2.6.2). The results of the scintillators' efficiencies run by run are graphically shown in Figure 39 and in full details in Appendix B.

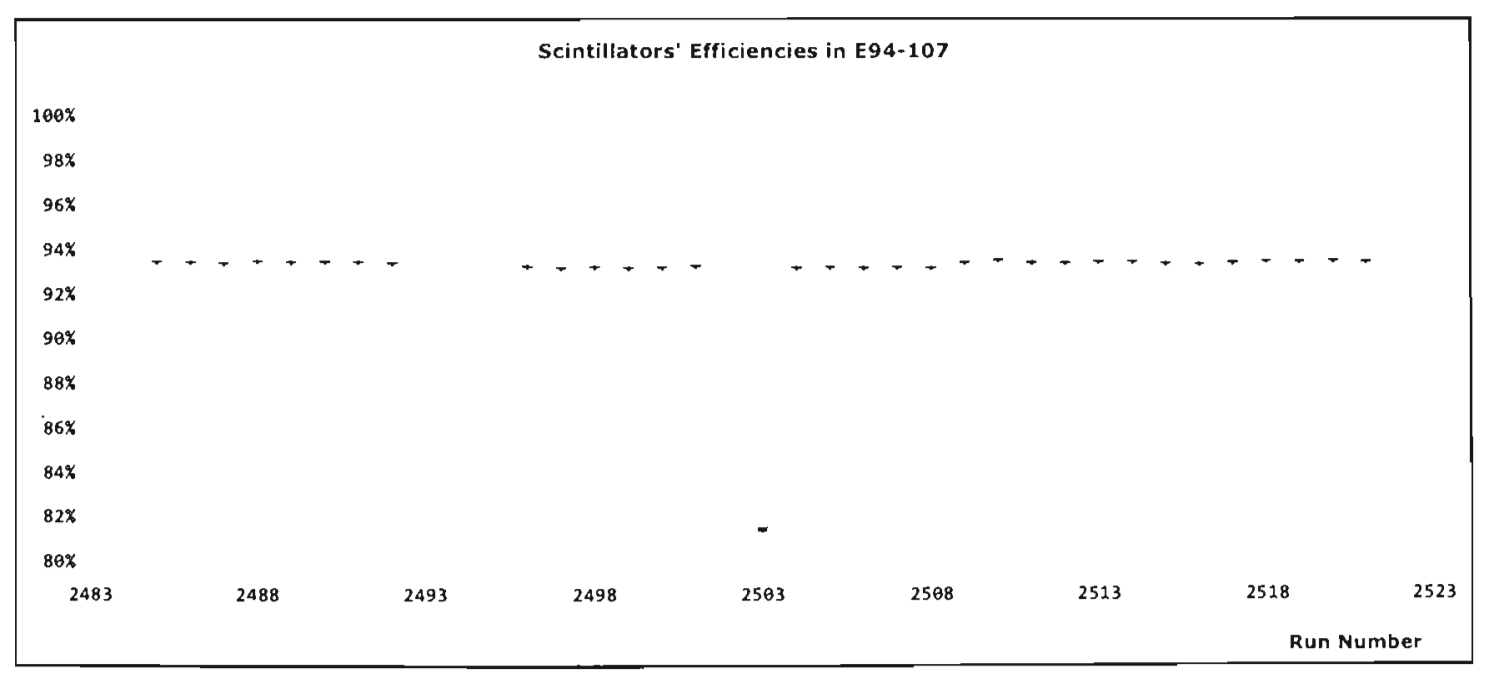

Figure 39: Scintillators's efficiencies per run number in E94-107. 
The summary of results is shown in Table 11. The run numbers are grouped into three different kinematics (momentum setting of the hadron). See Section 4.9 for details on how uncertainties were determined.

\begin{tabular}{|c|c|c|}
\hline Run Number & Scint_eff & Uncert. \\
\hline KIN_1 & $p_{K}=1.8665 \mathrm{GeV} / \mathrm{c}$ & \\
\hline 2485 & 0.93576 & 0.00002 \\
\hline 2486 & 0.93556 & 0.00002 \\
\hline 2487 & 0.93493 & 0.00002 \\
\hline 2488 & 0.93589 & 0.00003 \\
\hline 2489 & 0.93546 & 0.00003 \\
\hline 2490 & 0.93573 & 0.00003 \\
\hline 2491 & 0.93544 & 0.00003 \\
\hline 2492 & 0.93501 & 0.00003 \\
\hline KIN 2 & $p_{K}=1.8218 \mathrm{GeV} / \mathrm{c}$ & \\
\hline 2496 & 0.93326 & 0.00003 \\
\hline 2497 & 0.93249 & 0.00003 \\
\hline 2498 & 0.93307 & 0.00003 \\
\hline 2499 & 0.93257 & 0.00003 \\
\hline 2500 & 0.93280 & 0.00003 \\
\hline 2501 & 0.93342 & 0.00003 \\
\hline 2503 & 0.81533 & 0.00065 \\
\hline 2504 & 0.93262 & 0.00003 \\
\hline 2505 & 0.93273 & 0.00003 \\
\hline 2506 & 0.93251 & 0.00004 \\
\hline 2507 & 0.93278 & 0.00003 \\
\hline 2508 & 0.93243 & 0.00003 \\
\hline KIN_3 & $p_{K}=1.9600 \mathrm{GeV} / \mathrm{c}$ & \\
\hline 2509 & 0.93490 & 0.00002 \\
\hline 2510 & 0.93585 & 0.00002 \\
\hline 2511 & 0.93474 & 0.00002 \\
\hline 2512 & 0.93466 & 0.00002 \\
\hline 2513 & 0.93511 & 0.00002 \\
\hline 2514 & 0.93511 & 0.00002 \\
\hline 2515 & 0.93431 & 0.00002 \\
\hline 2516 & 0.93400 & 0.00003 \\
\hline 2517 & 0.93474 & 0.00002 \\
\hline 2518 & 0.93536 & 0.00002 \\
\hline 2519 & 0.93518 & 0.00002 \\
\hline 2520 & 0.93561 & 0.00002 \\
\hline 2521 & 0.93515 & 0.00002 \\
\hline
\end{tabular}

Table 11: Scintillators' efficiencies and uncertainties per run number. 


\subsection{Vertical drift chamber efficiencies}

The VDCs are the first detectors that particles get through in the detector stacks. The main reason to have these VDC detectors in front of the rest of detectors is to minimize undesired multiple scattering. Detector inefficiencies cause particles to not fire triggers, which are then missed from the data sample. Inefficiencies in the drift chambers (hardware) or tracking algorithms (software) both separately reject events. Even if a trigger is formed there will be some events where the information is too incomplete to reconstruct a particle track. The main source of these types of inefficiencies is events in which too many or too few wires fire in the drift chambers. It is usual for VDC detectors that any real particle going through the VDCs would produce three or more hits in each of the four planes. Therefore, a cut was applied to the number of wires that give a signal for a given event (multiplicity variable) in all four planes (U1, U2, V1, V2) for both hadron and electron arms. The cut applied was such that events that fired between 2 and 20 wires in each of the 4 wire planes were considered for the analysis.

The VDC efficiency represents the ratio of good events to the sum of good events and undetected events. The cross section requires the true number of events so the inverse of the efficiency is used as a correction factor for the yield to determine the cross section (see Section 4.8).

The number of particles that hit all planes is determined by means of the multiplicities on each individual plane for any VDC by:

$$
\mathrm{N}_{1234}=\mathrm{M}_{1} \cdot \mathrm{M}_{2} \cdot \mathrm{M}_{3} \cdot \mathrm{M}_{4}
$$


where the multiplication represents the logical AND operator. $M_{k}$ represents multiplicity and the indices 1, 2, 3 and 4 denote the planes $\mathrm{U} 1, \mathrm{~V} 1, \mathrm{U} 2$ and V2, respectively. The generic equation of the number of planes hit in any of the 3 VDC planes can be taken as:

$$
\mathrm{N}_{\mathrm{ijk}}=\mathrm{M}_{\mathrm{i}} \cdot \mathrm{M}_{\mathrm{j}} \cdot \mathrm{M}_{\mathrm{k}},(\mathrm{i}<\mathrm{j}<\mathrm{k})
$$

The tracking efficiency is determined by dividing the number of good 1-track particles by the number of hits in all planes given by (37). The number of good 1-track particles is the number of particles with a single track that made the detector fire. Therefore the efficiency for tracking can be expressed as (for any arm) [64]:

$$
\mathrm{E}_{\mathrm{VDC}}^{\mathrm{T}}=\frac{\mathrm{M}_{1} \cdot \mathrm{M}_{2} \cdot \mathrm{M}_{3} \cdot \mathrm{M}_{4} \cdot 1 \_ \text {Track }}{\mathrm{M}_{1} \cdot \mathrm{M}_{2} \cdot \mathrm{M}_{3} \cdot \mathrm{M}_{4}}
$$

The following plots in Figures 40 and 41 show the histograms of number of counts vs. number of hit wires per plane in the left arm and the right arm, and two peaks can be observed. The VDCs were set in such a way that when one particle is detected traversing them, is because around 5 wires get hit. Therefore, with less likehood, when two particles are detected traversing them, is because around 10 wires get hit. 

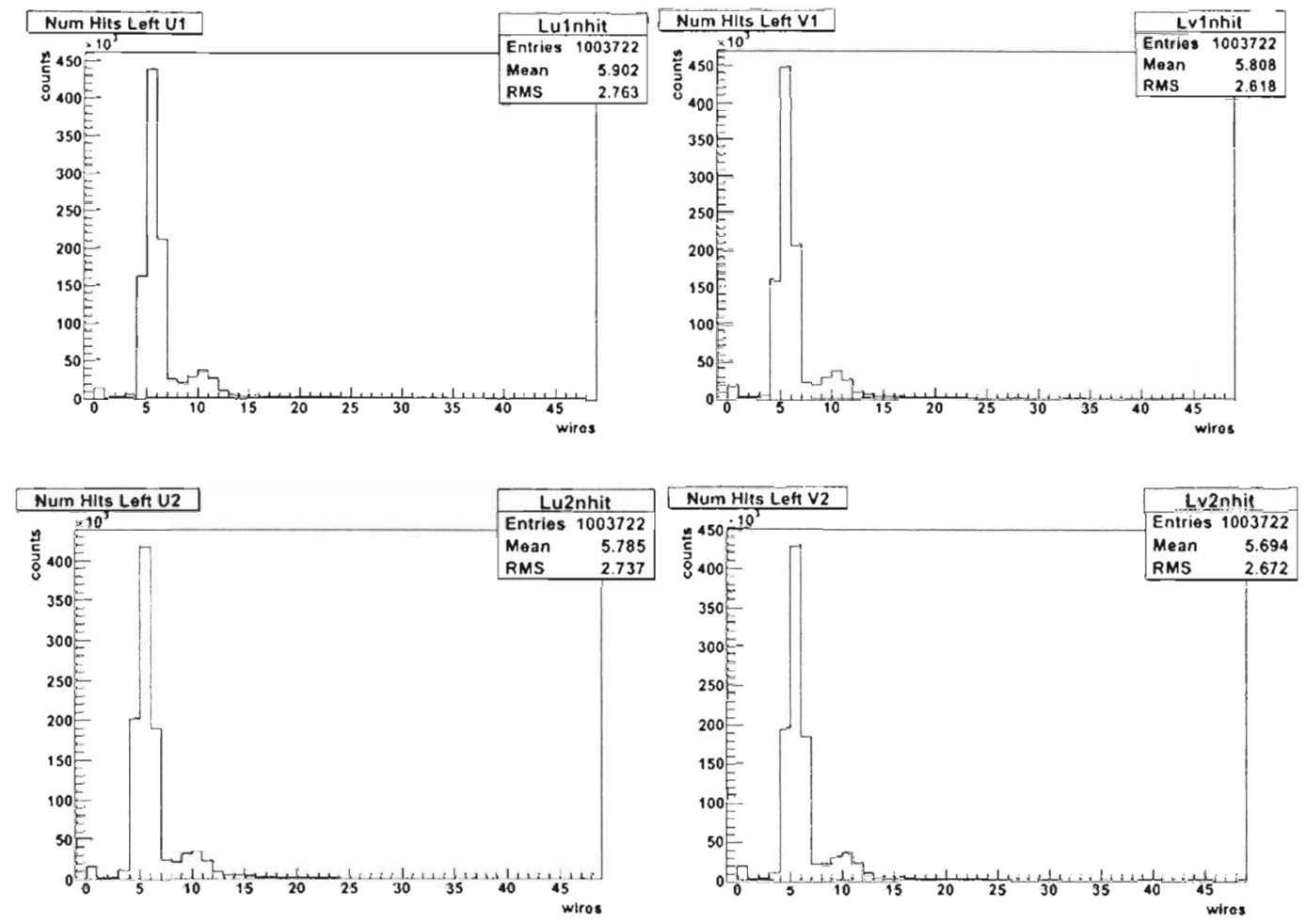

Figure 40: Number of hits on the left VDC planes for run 2509. Peak at 5 wires reflects one detected particle, peak at 10 wires reflects two detected particles simultaneously.

The single plane firing efficiency $\left(E_{\mathrm{VDC}}^{\mathrm{F}}\right)$ represents the probability a plane has been fired and it is defined as the ratio of the particles that fired all planes to those that fired at least three planes but without taking into account the plane for which the firing efficiency is being determined.

$$
E_{V D C_{1}}^{F}=\frac{N_{i j k l}}{N_{i j k}}
$$

Therefore, the total efficiency in the VDC either in the hadron arm or the electron arm can be define as the product (dependent probabilities) given by: 


$$
\mathrm{E}_{\mathrm{VDC}}^{\mathrm{F}}=\prod_{\mathrm{k}=1}^{4} \mathrm{E}_{\mathrm{VDC}}^{\mathrm{F}}
$$

Once the firing efficiency and the tracking efficiency for each arm are determined then the total VDC efficiency for each arm can be measured as the product:

$$
\begin{aligned}
& \mathrm{E}_{\mathrm{VDC}}^{\mathrm{e}}=\mathrm{E}_{\mathrm{VDC}}^{\mathrm{e}, \mathrm{F}} \times \mathrm{E}_{\mathrm{VDC}}^{\mathrm{e}, \mathrm{T}} \\
& \mathrm{E}_{\mathrm{VDC}}^{\mathrm{H}}=\mathrm{E}_{\mathrm{VDC}}^{\mathrm{H}, \mathrm{F}} \times \mathrm{E}_{\mathrm{VDC}}^{\mathrm{H}, \mathrm{T}}
\end{aligned}
$$

where e denotes electron arm and $\mathrm{H}$ denotes hadron arm.

Finally, the total VDC efficiency with the hadron VDC detector and the electron VDC detector combined results from multiplying (42) and (43)

$$
\mathrm{E}_{\mathrm{VDC}}=\mathrm{E}_{\mathrm{vDC}}^{\mathrm{e}} \times \mathrm{E}_{\mathrm{vDC}}^{\mathrm{H}}
$$

Figure 42 shows the VDC efficiencies plotted vs. the run number in E94-107. The uncertainty bars are very small and can barely be seen in the graph. In addition, Tables 12 and 13 show a summary of the main VDC efficiencies with their uncertainties. See Section 4.9 for details on how uncertainties are determined. Extra information on the VDC analysis can be found in Appendix C. 

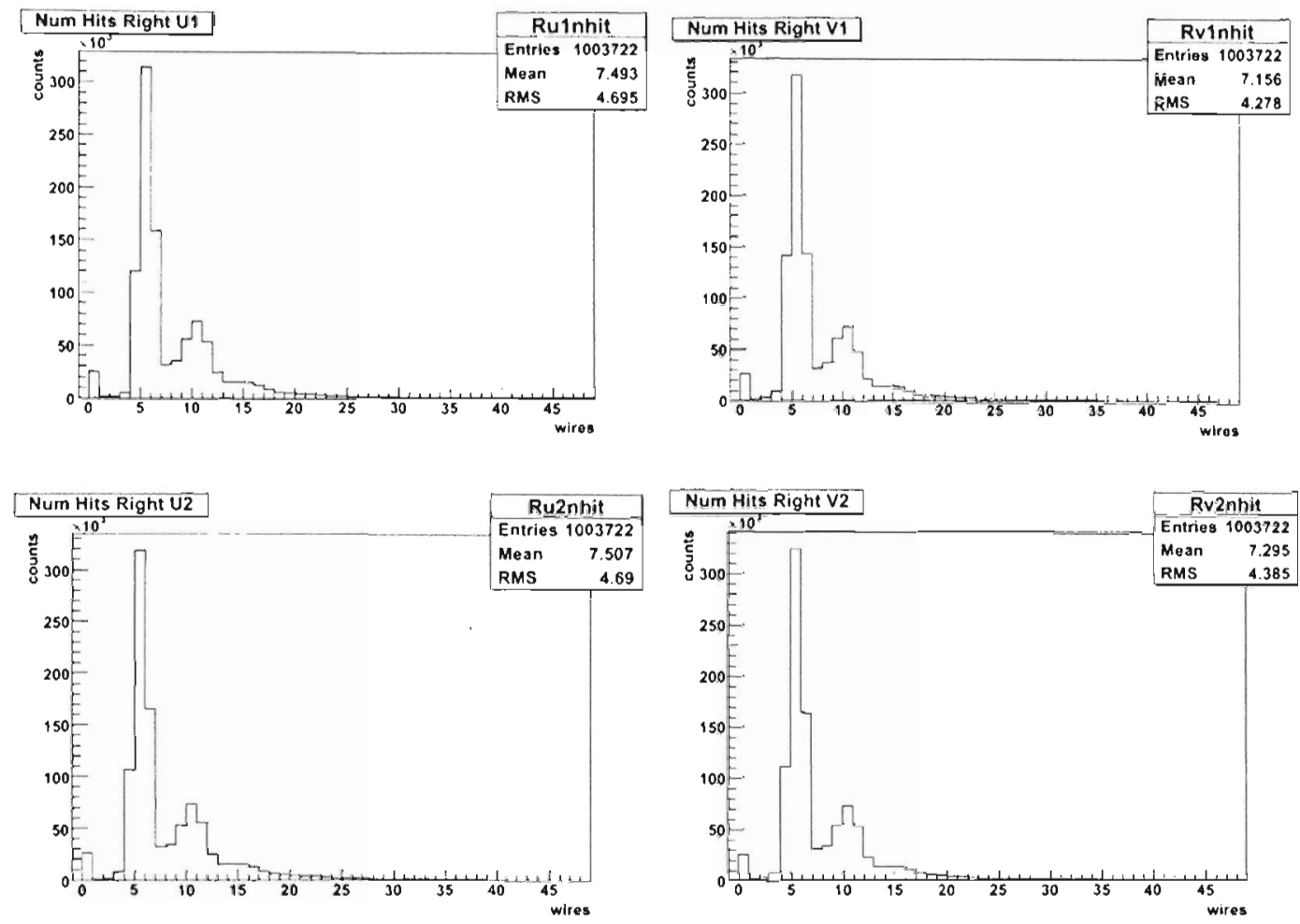

Figure 41: Number of hits on the right VDC planes for run 2509. Peak at 5 wires reflects one detected particle, peak at 10 wires reflects two detected particles simultaneously.

VDC Efficiencies in E94-107

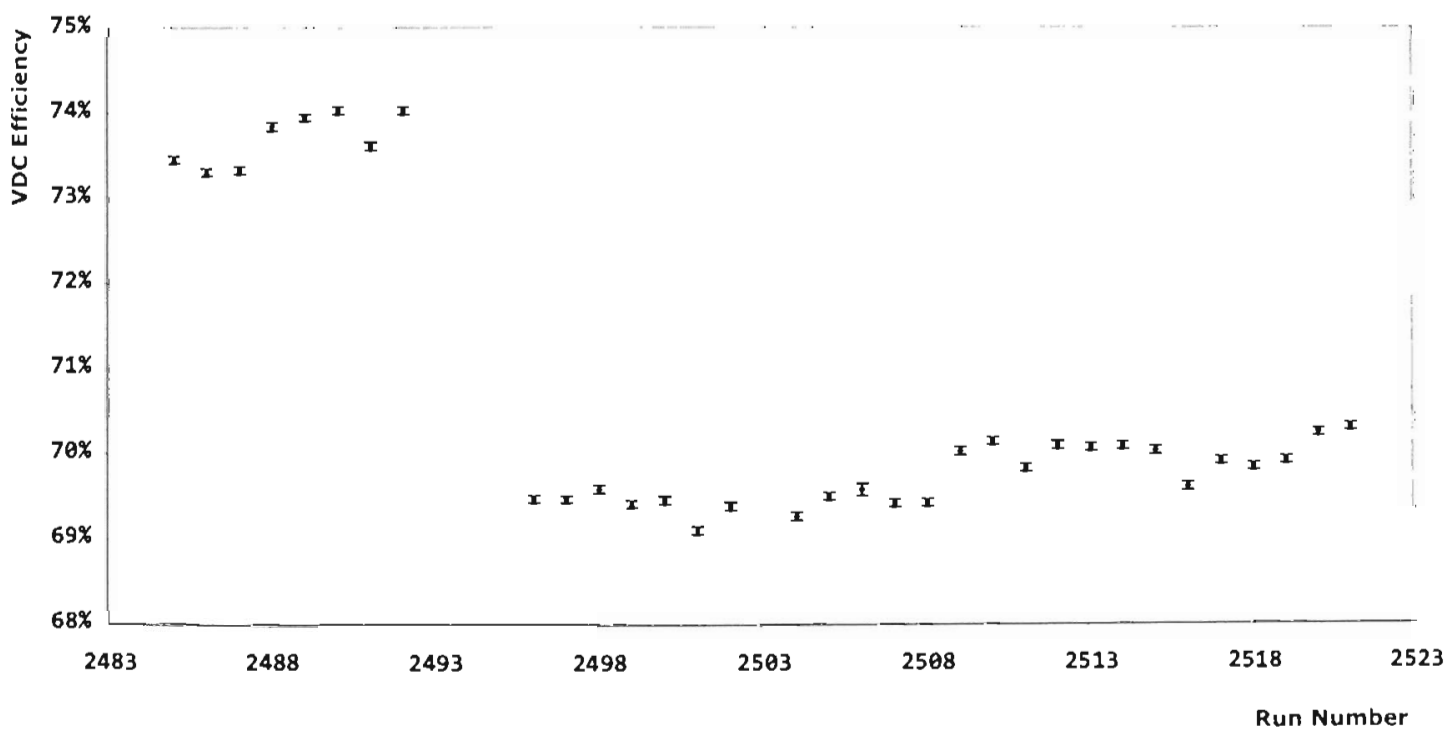

Figure 42: VDC efficiencies in E94-107. 


\begin{tabular}{|c|c|c|c|c|c|c|c|}
\hline RUN & $\begin{array}{l}\text { VDC } \\
\text { eff. }\end{array}$ & $\begin{array}{c}\text { VDC eff. } \\
\mathrm{R}\end{array}$ & $\begin{array}{c}\text { VDC eff. } \\
\mathrm{L}\end{array}$ & $\mathrm{R}$ tracking & $\begin{array}{c}\mathrm{L} \\
\text { tracking }\end{array}$ & $\begin{array}{l}\text { R firing } \\
\text { eff. }\end{array}$ & $\begin{array}{l}\text { L firing } \\
\text { eff. }\end{array}$ \\
\hline $\mathrm{K} I \mathrm{~N}_{-} 1$ & \multicolumn{2}{|c|}{$\mathrm{p}=1.8665 \mathrm{GeV} / \mathrm{c}$} & & & & & \\
\hline 2485 & 0.7344 & 0.8049 & 0.9124 & 0.8311 & 0.9329 & 0.9685 & 0.9780 \\
\hline 2486 & 0.7329 & 0.8042 & 0.9114 & 0.8302 & 0.9318 & 0.9686 & 0.9781 \\
\hline 2487 & 0.7332 & 0.8038 & 0.9121 & 0.8299 & 0.9326 & 0.9686 & 0.9781 \\
\hline 2488 & 0.7383 & 0.8074 & 0.9144 & 0.8336 & 0.9335 & 0.9687 & 0.9795 \\
\hline 2489 & 0.7394 & 0.8085 & 0.9146 & 0.8339 & 0.9340 & 0.9694 & 0.9792 \\
\hline 2490 & 0.7402 & 0.8088 & 0.9152 & 0.8349 & 0.9341 & 0.9687 & 0.9798 \\
\hline 2491 & 0.7360 & 0.8057 & 0.9135 & 0.8318 & 0.9328 & 0.9686 & 0.9793 \\
\hline 2492 & 0.7402 & 0.8086 & 0.9154 & 0.8343 & 0.9344 & 0.9691 & 0.9797 \\
\hline KIN_2 & \multicolumn{2}{|c|}{$\mathrm{p}=1.8218 \mathrm{GeV} / \mathrm{c}$} & & & & & \\
\hline 2496 & 0.6947 & 0.7747 & 0.8968 & 0.8028 & 0.9172 & 0.9650 & 0.9778 \\
\hline 2497 & 0.6946 & 0.7750 & 0.8963 & 0.8031 & 0.9170 & 0.9650 & 0.9775 \\
\hline 2498 & 0.6958 & 0.7761 & 0.8966 & 0.8041 & 0.9173 & 0.9652 & 0.9775 \\
\hline 2499 & 0.6940 & 0.7748 & 0.8957 & 0.8031 & 0.9167 & 0.9649 & 0.9771 \\
\hline 2500 & 0.6945 & 0.7748 & 0.8965 & 0.8029 & 0.9170 & 0.9649 & 0.9776 \\
\hline 2501 & 0.6910 & 0.7721 & 0.8949 & 0.8002 & 0.9158 & 0.9649 & 0.9772 \\
\hline 2502 & 0.6938 & 0.7748 & 0.8954 & 0.8028 & 0.9164 & 0.9651 & 0.9771 \\
\hline \multicolumn{8}{|l|}{2503} \\
\hline 2504 & 0.6926 & 0.7733 & 0.8956 & 0.8015 & 0.9165 & 0.9649 & 0.9772 \\
\hline 2505 & 0.6949 & 0.7759 & 0.8957 & 0.8039 & 0.9166 & 0.9651 & 0.9772 \\
\hline 2506 & 0.6957 & 0.7761 & 0.8965 & 0.8038 & 0.9173 & 0.9655 & 0.9774 \\
\hline 2507 & 0.6942 & 0.7749 & 0.8958 & 0.8033 & 0.9164 & 0.9647 & 0.9775 \\
\hline 2508 & 0.6942 & 0.7748 & 0.8960 & 0.8032 & 0.9168 & 0.9647 & 0.9773 \\
\hline $\mathrm{KIN}_{-} 3$ & \multicolumn{2}{|c|}{$\mathrm{p}=1.9600 \mathrm{GeV} / \mathrm{c}$} & & & & & \\
\hline 2509 & 0.7002 & 0.7749 & 0.9036 & 0.8030 & 0.9241 & 0.9651 & 0.9777 \\
\hline 2510 & 0.7013 & 0.7756 & 0.9042 & 0.8036 & 0.9251 & 0.9652 & 0.9775 \\
\hline 2511 & 0.6982 & 0.7732 & 0.9031 & 0.8014 & 0.9237 & 0.9647 & 0.9777 \\
\hline 2512 & 0.7009 & 0.7756 & 0.9036 & 0.8036 & 0.9245 & 0.9652 & 0.9774 \\
\hline 2513 & 0.7006 & 0.7754 & 0.9036 & 0.8038 & 0.9244 & 0.9647 & 0.9774 \\
\hline 2514 & 0.7008 & 0.7751 & 0.9041 & 0.8032 & 0.9250 & 0.9650 & 0.9774 \\
\hline 2515 & 0.7003 & 0.7749 & 0.9036 & 0.8032 & 0.9244 & 0.9648 & 0.9775 \\
\hline 2516 & 0.6960 & 0.7713 & 0.9024 & 0.7998 & 0.9237 & 0.9644 & 0.9770 \\
\hline 2517 & 0.6990 & 0.7740 & 0.9031 & 0.8021 & 0.9241 & 0.9650 & 0.9773 \\
\hline 2518 & 0.6983 & 0.7732 & 0.9031 & 0.8012 & 0.9240 & 0.9651 & 0.9774 \\
\hline 2519 & 0.6991 & 0.7739 & 0.9034 & 0.8021 & 0.9243 & 0.9648 & 0.9774 \\
\hline 2520 & 0.7024 & 0.7766 & 0.9044 & 0.8044 & 0.9253 & 0.9654 & 0.9774 \\
\hline 2521 & 0.7030 & 0.7771 & 0.9046 & 0.8048 & 0.9254 & 0.9655 & 0.9775 \\
\hline
\end{tabular}

Table 12: VDC efficiencies in E94-107 for the three different kinematics. Firing and tracking efficiencies for left (L) and right (R) arms. 


\begin{tabular}{|c|c|c|c|c|c|c|c|}
\hline RUN & L firing eff & $\mathrm{R}$ firing eff & $\mathrm{L}$ track eff & $\mathrm{R}$ track eff & VDC eff L & VDC_eff R & VDC eff \\
\hline & uncertainty & uncertainty & uncertainty & uncertainty & uncertainty & uncertainty & uncertainty \\
\hline \multicolumn{8}{|l|}{ KIN_1 } \\
\hline 2485 & 0.0001 & 0.0002 & 0.0003 & 0.0004 & 0.0003 & 0.0004 & 0.0004 \\
\hline 2486 & 0.0001 & 0.0002 & 0.0003 & 0.0004 & 0.0003 & 0.0004 & 0.0004 \\
\hline 2487 & 0.0001 & 0.0002 & 0.0003 & 0.0004 & 0.0003 & 0.0004 & 0.0004 \\
\hline 2488 & 0.0002 & 0.0002 & 0.0003 & 0.0005 & 0.0003 & 0.0005 & 0.0005 \\
\hline 2489 & 0.0001 & 0.0002 & 0.0003 & 0.0004 & 0.0003 & 0.0004 & 0.0004 \\
\hline 2490 & 0.0001 & 0.0002 & 0.0003 & 0.0004 & 0.0003 & 0.0004 & 0.0004 \\
\hline 2491 & 0.0001 & 0.0002 & 0.0003 & 0.0004 & 0.0003 & 0.0004 & 0.0004 \\
\hline 2492 & 0.0001 & 0.0002 & 0.0003 & 0.0004 & 0.0003 & 0.0004 & 0.0004 \\
\hline \multicolumn{8}{|l|}{ KIN_2 } \\
\hline 2496 & 0.0002 & 0.0002 & 0.0003 & 0.0004 & 0.0003 & 0.0004 & 0.0005 \\
\hline 2497 & 0.0002 & 0.0002 & 0.0003 & 0.0004 & 0.0003 & 0.0004 & 0.0005 \\
\hline 2498 & 0.0002 & 0.0002 & 0.0003 & 0.0004 & 0.0003 & 0.0004 & 0.0005 \\
\hline 2499 & 0.0002 & 0.0002 & 0.0003 & 0.0004 & 0.0003 & 0.0004 & 0.0005 \\
\hline 2500 & 0.0001 & 0.0002 & 0.0003 & 0.0004 & 0.0003 & 0.0004 & 0.0005 \\
\hline 2501 & 0.0002 & 0.0002 & 0.0003 & 0.0004 & 0.0003 & 0.0005 & 0.0005 \\
\hline 2502 & 0.0002 & 0.0002 & 0.0003 & 0.0004 & 0.0003 & 0.0004 & 0.0005 \\
\hline \multicolumn{8}{|l|}{2503} \\
\hline 2504 & 0.0002 & 0.0002 & 0.0003 & 0.0004 & 0.0003 & 0.0004 & 0.0005 \\
\hline 2505 & 0.0002 & 0.0002 & 0.0003 & 0.0004 & 0.0003 & 0.0004 & 0.0005 \\
\hline 2506 & 0.0002 & 0.0003 & 0.0004 & 0.0007 & 0.0005 & 0.0007 & 0.0007 \\
\hline 2507 & 0.0002 & 0.0002 & 0.0003 & 0.0004 & 0.0003 & 0.0004 & 0.0005 \\
\hline 2508 & 0.0002 & 0.0002 & 0.0003 & 0.0004 & 0.0003 & 0.0004 & 0.0005 \\
\hline \multicolumn{8}{|l|}{ KIN_3 } \\
\hline 2509 & 0.0001 & 0.0002 & 0.0003 & 0.0004 & 0.0003 & 0.0004 & 0.0005 \\
\hline 2510 & 0.0002 & 0.0002 & 0.0003 & 0.0004 & 0.0003 & 0.0004 & 0.0005 \\
\hline 2511 & 0.0001 & 0.0002 & 0.0003 & 0.0004 & 0.0003 & 0.0004 & 0.0005 \\
\hline 2512 & 0.0002 & 0.0002 & 0.0003 & 0.0004 & 0.0003 & 0.0004 & 0.0005 \\
\hline 2513 & 0.0002 & 0.0002 & 0.0003 & 0.0004 & 0.0003 & 0.0004 & 0.0005 \\
\hline 2514 & 0.0002 & 0.0002 & 0.0003 & 0.0004 & 0.0003 & 0.0004 & 0.0005 \\
\hline 2515 & 0.0002 & 0.0002 & 0.0003 & 0.0004 & 0.0003 & 0.0004 & 0.0005 \\
\hline 2516 & 0.0002 & 0.0002 & 0.0003 & 0.0004 & 0.0003 & 0.0004 & 0.0005 \\
\hline 2517 & 0.0002 & 0.0002 & 0.0003 & 0.0004 & 0.0003 & 0.0004 & 0.0005 \\
\hline 2518 & 0.0002 & 0.0002 & 0.0003 & 0.0004 & 0.0003 & 0.0004 & 0.0005 \\
\hline 2519 & 0.0002 & 0.0002 & 0.0003 & 0.0004 & 0.0003 & 0.0004 & 0.0005 \\
\hline 2520 & 0.0002 & 0.0002 & 0.0003 & 0.0004 & 0.0003 & 0.0004 & 0.0005 \\
\hline 2521 & 0.0002 & 0.0002 & 0.0003 & 0.0004 & 0.0003 & 0.0004 & 0.0005 \\
\hline
\end{tabular}

Table 13: VDC efficiency uncertainties in E94-107 for the three different kinematics. Firing and tracking efficiency uncertainties for left (L) and right (R) arms. 


\subsection{Deadtime correction}

In general for most of the detector systems, there is a minimum amount of time that must separate two events in order for the two to be recorded as separate events. In some cases, processes in the detectors themselves may set the limiting time, and in other cases the limit may arise in the associated electronics. This minimum time separation is usually called the deadtime of the system. Conversely, the livetime is the total time a detector system runs minus the deadtime. Therefore, deadtime $=1$-livetime, where deadtime and livetime are taken as ratios with respect to total time.

In E94-107, the scalers (counters) and event stream are used to determine the deadtime correction for the analysis of the experiment by computing the ratio of accepted triggers to input triggers [65]. Information on the livetime is registered automatically on the end-of-the-run statement at the end of each run. The livetime registered accounts for both deadtimes combined; the electronics deadtime and the computer deadtime. In general it is important to compute these two different deadtimes separately to avoid errors in the deadtime correction. The scalers are normally used to compute the livetime for three different situations; livetime[+], livetime[-] and livetime[0]. The livetime[+] and livetime[-], refer to the two opposite longitudinal electron polarizations as determined by the circular laser polarization of the laser used to produce electrons, that later, are sent to the accelerator through injector. The livetime[0] refers to the sum of the two polarizations, or a net zero polarization. The different polarization of the laser knock out electrons which are produced with different polarizations and then have their spin turned longitudinal (or anti-longitudinal). However, when electrons go through the accelerator's 
arcs the magnetic precession can change the direction of the electron polarization. Therefore, to determine the livetime (1-deadtime) the information on the scalers was averaged and the related systematic uncertainty was estimated by taking the standard deviation of the three different measurements. The correction factor will be the inverse of the livetime as described above.

\begin{tabular}{|c|c|c|c|c|c|c|c|}
\hline KIN 1 & liveTIME $(\%)$ & & & & & \multicolumn{2}{|c|}{ Correction Factor } \\
\hline RUN & {$[0]$} & {$[+]$} & {$[-]$} & $\begin{array}{c}\text { Mean } \\
(\%)\end{array}$ & $\begin{array}{l}\text { Stand. } \\
\text { D. }(\%)\end{array}$ & 1/Mean & Uncertainty \\
\hline 2485 & $91.5 \mathrm{I}$ & 91.7 & 91.69 & 91.63 & 0.1069 & 1.0913 & 0.0013 \\
\hline 2486 & 91.44 & 91.61 & 91.66 & 91.57 & 0.1153 & 1.0921 & 0.0014 \\
\hline 2487 & 91.42 & 91.63 & 91.62 & 91.56 & 0.1185 & 1.0922 & 0.0014 \\
\hline 2488 & 88.92 & 89.09 & 89.18 & 89.06 & 0,1320 & 1.1228 & 0.0017 \\
\hline 2489 & 89.08 & 89.29 & 89.29 & 89.22 & 0.1212 & 1.1208 & 0.0015 \\
\hline 2490 & 89.19 & 89.43 & 89.36 & 89.33 & 0.1234 & 1.1195 & 0.0015 \\
\hline 2491 & 88.58 & 88.84 & 88.72 & 88.71 & 0.1301 & 1.1272 & 0.0017 \\
\hline 2492 & 89.09 & 89.34 & 89.28 & 89.24 & 0.1305 & 1.1206 & 0.0016 \\
\hline \multicolumn{8}{|l|}{ KIN_2 } \\
\hline RUN & {$[0]$} & {$[+]$} & {$[-]$} & & & & \\
\hline 2496 & 86.99 & 87.18 & 87.18 & 87.12 & 0.1097 & 1.1479 & 0.0014 \\
\hline 2497 & 87 & 87.17 & 87.22 & 87.13 & 0.1153 & 1.1477 & 0.0015 \\
\hline 2498 & 87 & 87.28 & 87.07 & 87.12 & 0.1457 & 1.1479 & 0.0019 \\
\hline 2499 & 86.83 & 86.99 & 87.05 & 86.96 & 0.1137 & 1.1500 & 0.0015 \\
\hline 2500 & 86.95 & 87.25 & 87.03 & 87.08 & 0.1553 & 1.1484 & 0.0020 \\
\hline 2501 & 86.51 & 86.7 & 86.71 & 86.64 & 0.1127 & 1.1542 & 0.0015 \\
\hline \multicolumn{8}{|l|}{2502} \\
\hline 2503 & 97.87 & 100 & 95.45 & 97.77 & 2.2765 & 1.0228 & 0.0238 \\
\hline 2504 & 86.68 & 86.86 & 86.87 & 86.80 & 0.1069 & 1.1520 & 0.0014 \\
\hline 2505 & 86.94 & 87.09 & 87.14 & 87.06 & 0.1041 & 1.1487 & 0.0014 \\
\hline 2506 & 86.87 & 87.03 & 87.09 & 87.00 & 0.1137 & 1.1495 & 0.0015 \\
\hline 2507 & 86.77 & 86.96 & 86.93 & 86.89 & 0.1021 & 1.1509 & 0.0014 \\
\hline 2508 & 86.84 & 87 & 87.07 & 86.97 & 0.1179 & 1.1498 & 0.0016 \\
\hline \multicolumn{8}{|l|}{ KIN_3 } \\
\hline RUN & {$[0]$} & {$[+]$} & {$[-]$} & & & & \\
\hline 2509 & 88.4 & 88.58 & 88.6 & 88.53 & 0.1102 & 1.1296 & 0.0014 \\
\hline 2510 & 88.8 & 88.96 & 89.04 & 88.93 & 0.1222 & 1.1244 & 0.0015 \\
\hline 2511 & 87.99 & 88.11 & 88.27 & 88.12 & 0.1405 & 1.1348 & 0.0018 \\
\hline 2512 & 88.69 & 88.85 & 88.91 & 88.82 & 0.1137 & 1.1259 & 0.0014 \\
\hline 2513 & 88.36 & 88.57 & 88.53 & 88.49 & 0.1115 & 1.1301 & 0.0014 \\
\hline 2514 & 88.34 & 88.48 & 88.59 & 88.47 & 0.1253 & 1.1303 & 0.0016 \\
\hline 2515 & 88.27 & 88.42 & 88.51 & 88.40 & 0.1212 & 1.1312 & 0.0016 \\
\hline 2516 & 88.7 & 88.98 & 88.82 & 88.83 & 0.1405 & 1.1257 & 0.0018 \\
\hline 2517 & 88.12 & 88.37 & 88.22 & 88.24 & 0.1258 & 1.1333 & 0.0016 \\
\hline 2518 & 88.05 & 88.23 & 88.25 & 88.18 & 0.1102 & 1.1341 & 0.0014 \\
\hline 2519 & 88.11 & 88.32 & 88.26 & 88.23 & 0.1082 & 1.1334 & 0.0014 \\
\hline 2520 & 88.5 & 88.67 & 88.69 & 88.62 & 0.1044 & 1.1284 & 0.0013 \\
\hline 2521 & 88.52 & 88.67 & 88.75 & 88.65 & 0.1168 & 1.1281 & 0.0015 \\
\hline
\end{tabular}

Table 14: Livetime (\%) and deadtime correction factors in E94-107 [66]. 
As shown in Table 14 the uncertainty on the livetime (1-deadtime) is quite small (see Figure 43). Therefore, this estimate will not imply a significant contribution in the overall uncertainty since it is very small compared to the uncertainties obtained from the aerogel threshold Cherenkov detector analysis in Section 4.4.

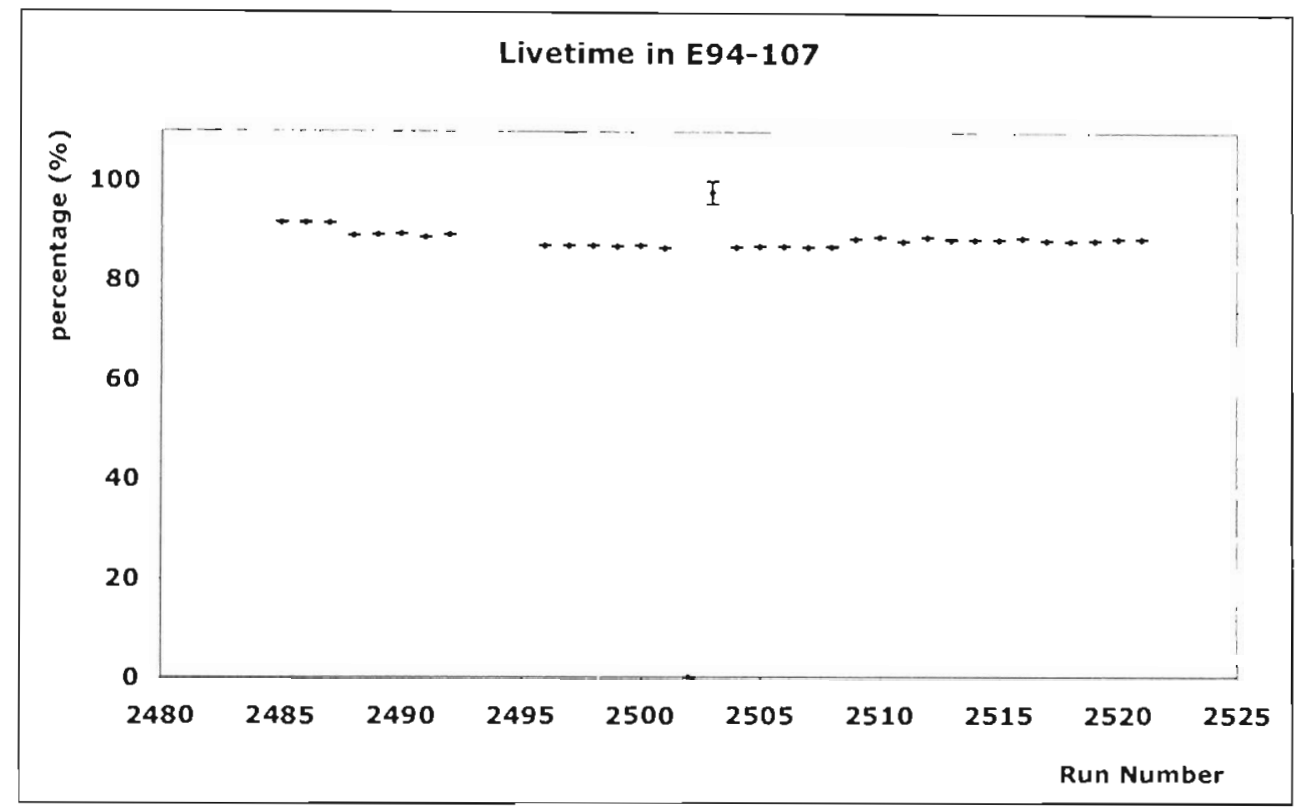

Figure 43: Livetime in E94-107 per run number.

\section{$3.7 \quad$ Target density}

In many experiments an important factor to consider during analysis is the target density fluctuation with the beam current fluctuation, raster size changes and fan speed. The density fluctuation can be observed by using luminosity monitors when colliding the electron beam on different targets since the luminosity is proportional to the density. D.S. 
Armstrong et al., showed that for the hydrogen cryotarget used in E94-107 and for currents of up to $100 \mu \mathrm{A}$, the target density remains constant (see Figures 44 and 45). Consequently, for E94-107 analysis there is no boiling correction to perform [67].

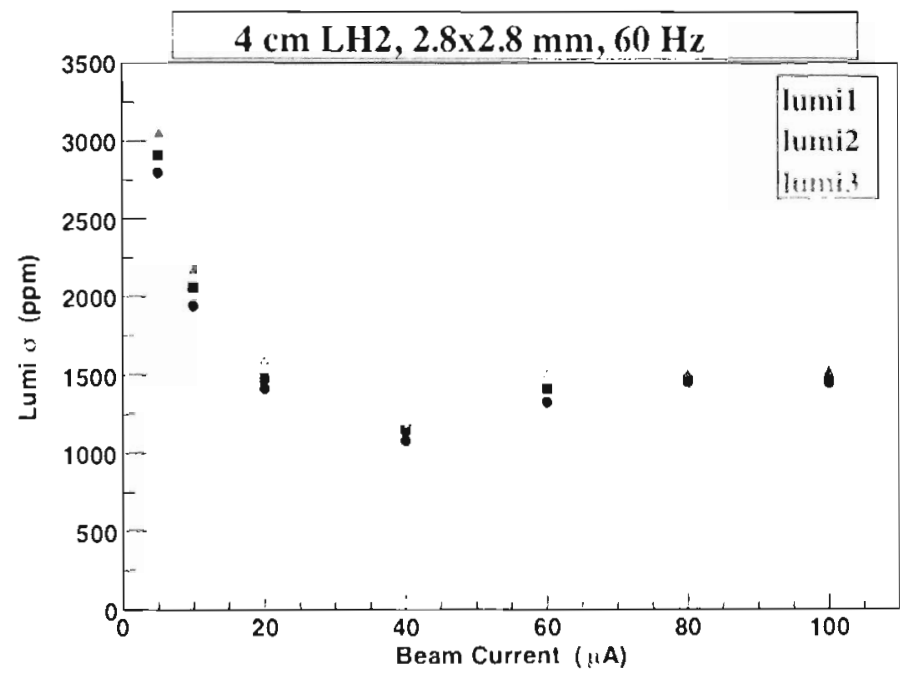

Figure 44: $\mathrm{LH}_{2}$ cryotarget density fluctuations vs. beam current, showing three data sets of independent measurements [67].

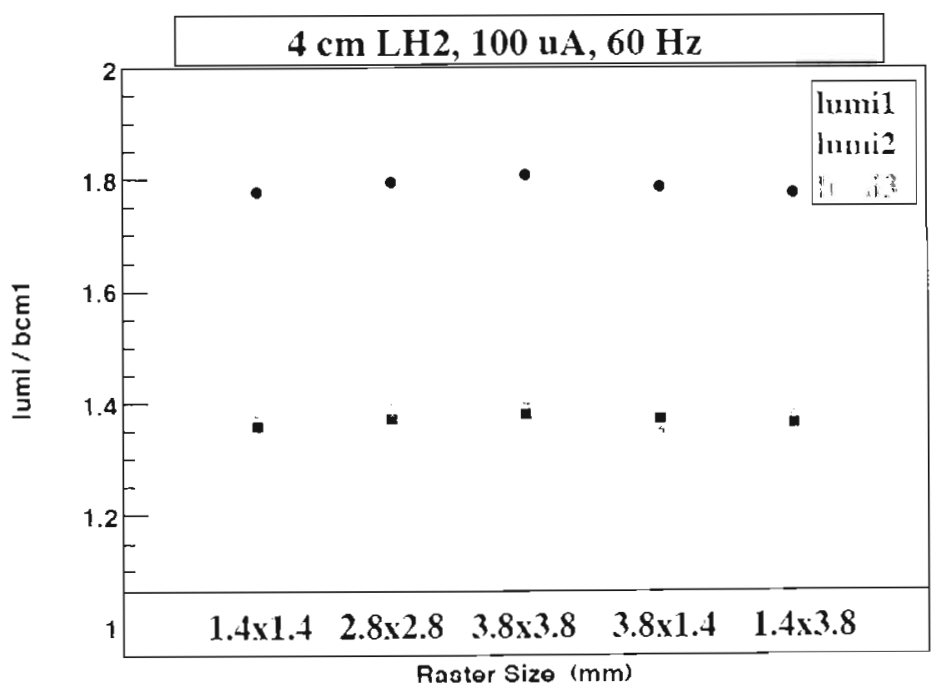

Figure 45: $\mathrm{LH}_{2}$ cryotarget fluctuation vs. raster size, showing three data sets of independent measurents in arbitrary units [67]. 
Although, the LH2 cryotarget density does not show dependence on beam currents around $100 \mu \mathrm{A}$ [67] as used in E94-107, a small uncertainty was assigned to account for the minimum variations among the different measurements reported by $\mathrm{D}$. Armstrong et al., [67]. The assigned target density uncertainty is $1 \%$ (see Table 48 ).

$4 \quad$ Data analysis

This section describes the data analysis used to determine all necessary pieces of information to extract the cross sections (which are given in Section 4.8) from the experimental measurements, and later, to do some data binning to observe the trend of the cross sections with respect to the main variables for the elementary reaction.

As mentioned before in previous chapters, three slightly different kinematic settings were used. They differ in the momentum setting of the left (kaon) spectrometer arm as shwon in Table 15.

\begin{tabular}{cc}
\hline Kinematics & L-arm momentum \\
\hline KIN_1 (runs 2485-2492) & $p=1.8665 \mathrm{GeV} / \mathrm{c}$ \\
KIN_2 (runs 2496-2508) & $p=1.8218 \mathrm{GeV} / \mathrm{c}$ \\
KIN_3 (runs 2509-2521) & $p=1.9600 \mathrm{GeV} / \mathrm{c}$ \\
\hline
\end{tabular}

Table 15: Kinematics in E94-107. 


\subsection{Particle identification}

In E94-107 the aerogel threshold Cherenkov detectors (A1 and A2) were the primary detectors used to discriminate kaons from other hadrons, e.g., pions and protons. As discussed in Section 2.6.5 concerning the PID conditions, Table 16 shows the cuts utilized in the aerogel detectors.

\begin{tabular}{ccc}
\hline Particle & $\begin{array}{c}\text { A1 cut } \\
\text { (channels) }\end{array}$ & $\begin{array}{c}\text { A2 cut } \\
\text { (channels) }\end{array}$ \\
\hline$K^{+}$ & $<10$ & $>20$ \\
$\pi^{+}$ & $>10$ & $>20$ and $<1100$ \\
$p^{+}$ & $<10$ & $<20$ \\
\hline
\end{tabular}

Table 16: Applied aerogel cuts for PID in E94-107.

The typical collected aerogel detector signals are plotted in Figures 46 (for A1) and 47 (for A2). 


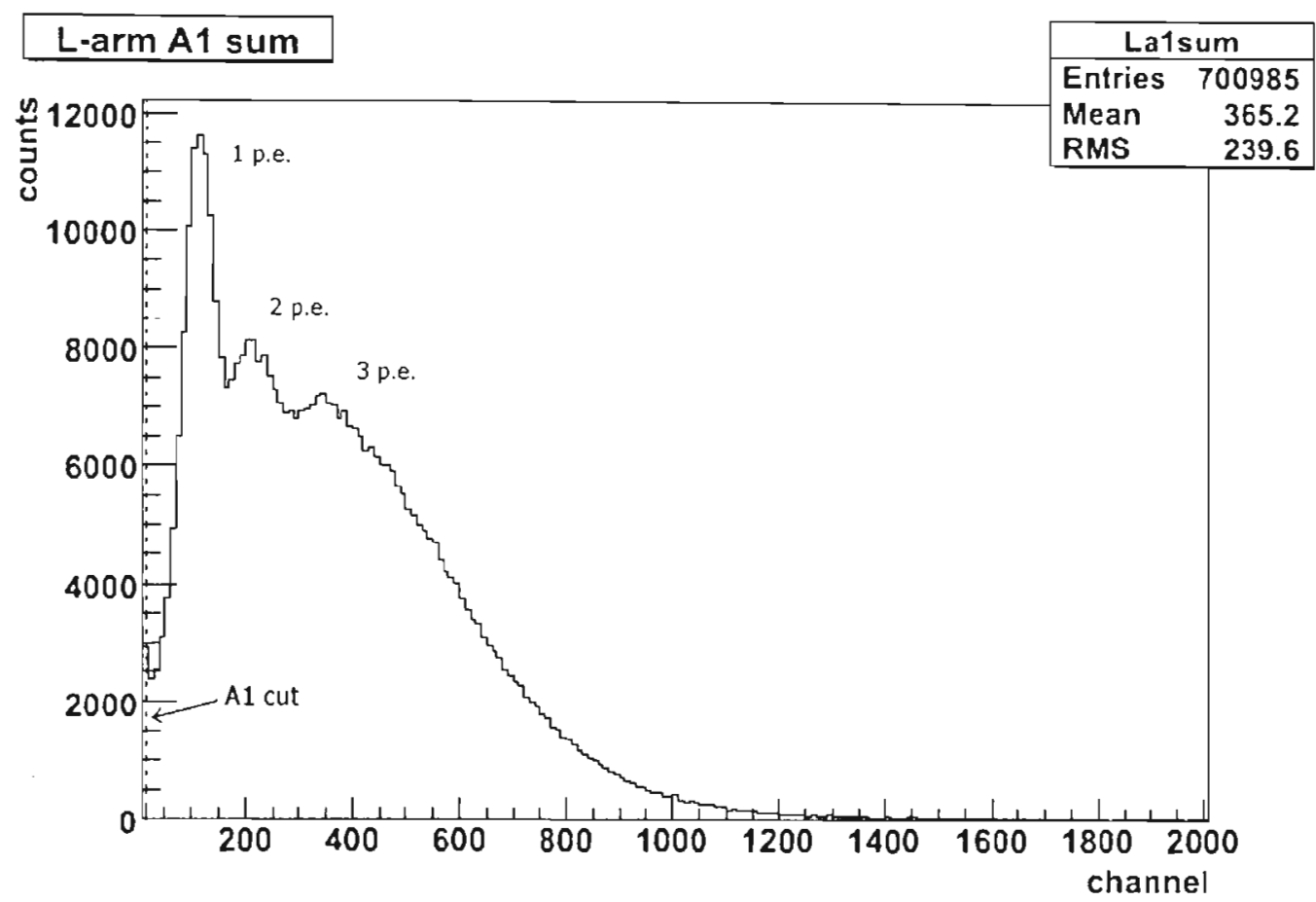

Figure 46: Al ADC signal in run 2485, showing the $1^{\text {st }}, 2^{\text {nd }}$ and $3^{\text {rd }}$ photoelectron peaks, and $\mathrm{Al}$ cut (dashed line) according to Table 16.

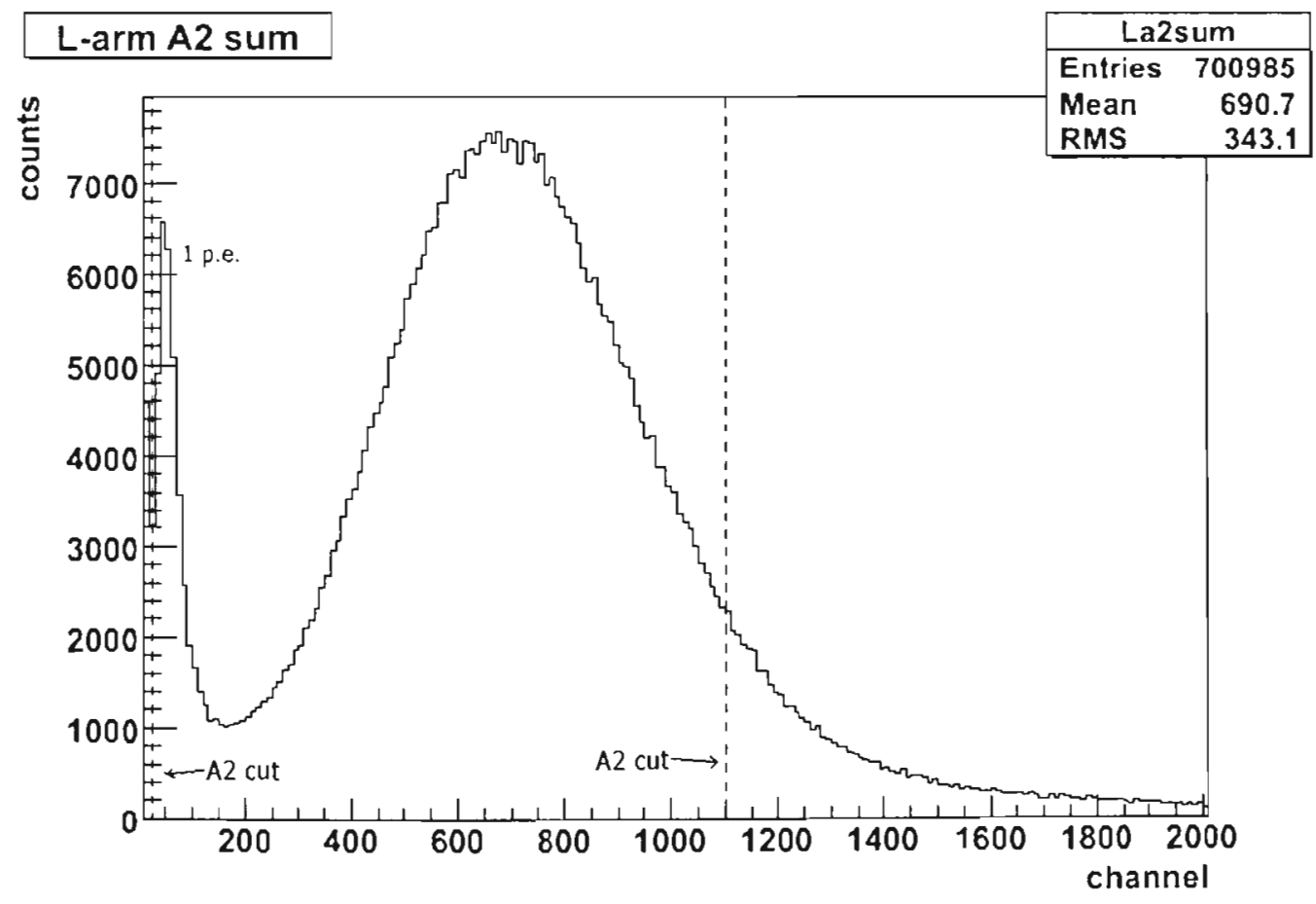

Figure 47: A2 ADC signal in run 2485, showing the $1^{\text {st }}$ photoelectron peak, and the two A2 cuts (dashed line) according to Table 16. 
In Figure 46 the single photoelectron $\left(1^{\text {st }}\right)$ peak was calibrated to the ADC channel 100, which yields approximately 4.5 photoelectrons in A1 for pions. It is worth remarking on the extreme sensitivity of the PMTs used in A1 that allow the second and the third photoelectron peaks to be distinguished. In Figure 47 the $1^{\text {st }}$ peak was calibrated to the ADC channel 50, which gives approximately 14 photoelectrons in A2 for kaons. Moreover, by variously combining the cuts shown in Table 16, the three different particles can be selected. The extra cut in $\mathrm{A} 2$ for pions $\left(\pi^{+}\right)$was found to give a clearer identification of pions, which might result a reduction of signal created by delta electrons in A2 (see Section 2.6.5).

On the other hand, for the $\mathrm{LH}_{2}$ target, the $\mathrm{RICH}$ detector played the role of an extra detector that was also used for PID. The presence of the RICH detector allowed clean samples of pions, kaons and protons to be determined, which could then in turn be used to measure the efficiency for A1 and A2. However the use of the RICH detector did result in a small loss in efficiency. The result is that the RICH was used to determine the efficiencies for $\mathrm{A} 1$ and $\mathrm{A} 2$ but not in the actual extraction of the cross sections. The cuts used for the RICH were as shown in Table 17.

\begin{tabular}{cc}
\hline Particle & RICH PID cuts (Cherenkov cone angles) \\
\hline$K^{+}$ & $0.621 \mathrm{rad}<\theta_{\mathrm{c}}<0.660 \mathrm{rad}$ \\
$\pi^{+}$ & $0.665 \mathrm{rad}<\theta_{\mathrm{c}}<0.695 \mathrm{rad}$ \\
$p^{+}$ & $0.498 \mathrm{rad}<\theta_{\mathrm{c}}<0.570 \mathrm{rad}$ \\
\hline
\end{tabular}

Table 17: RICH PID cuts in E94-107. 
Table 17 shows the cuts imposed at the different angles of the emitted Cherenkov radiation cones for the three different particles. In addition, two more common cuts for all particles that are related to the minimum ionizing point in the center of the Cherenkov rings and the tracking of the particles in the RICH wire chambers include are shown in Table 18.

\begin{tabular}{c}
\hline Number of photoelectrons (n.p.e.) $>2$ \\
\hline $50 \mathrm{~mm}<x_{\text {tracking }}<1900 \mathrm{~mm}, 50 \mathrm{~mm}<y_{\text {tracking }}<300 \mathrm{~mm}$ \\
\hline
\end{tabular}

Table 18: Additional RICH cuts used in E94-107.

Table 18 shows several cuts combined in the RICH. The first cut (line 1) on the minimum number of photoelectrons produced at the point the particle is going through the detector, which served to ensure that a particle has been detected. The second cut (line 2) on the $x$ and $y$ tracking variables sets the area over the detector where detected particles will be accepted as such. The RICH detector has its own wire tracking system that locates the position of the particle going through the detector. The values of the RICH cuts were provided by F. Cusanno, which have been extensively used in the analysis of the carbon, oxygen, and beryllium targets by the Hall A hypernuclear collaboration [58]. On the other hand, the particles that are identified in the left arm must be in coincidence with electrons in the right arm. However, in the right arm there are not only electrons but also negative pions that pass through the spectrometer. To discriminate electrons from negative pions an additional PID cut is used for the $\mathrm{CO}_{2}$ gas Cherenkov detector. The typical ADC signal of the gas Cherenkov detector in the right arm is shown in Figure 48: 


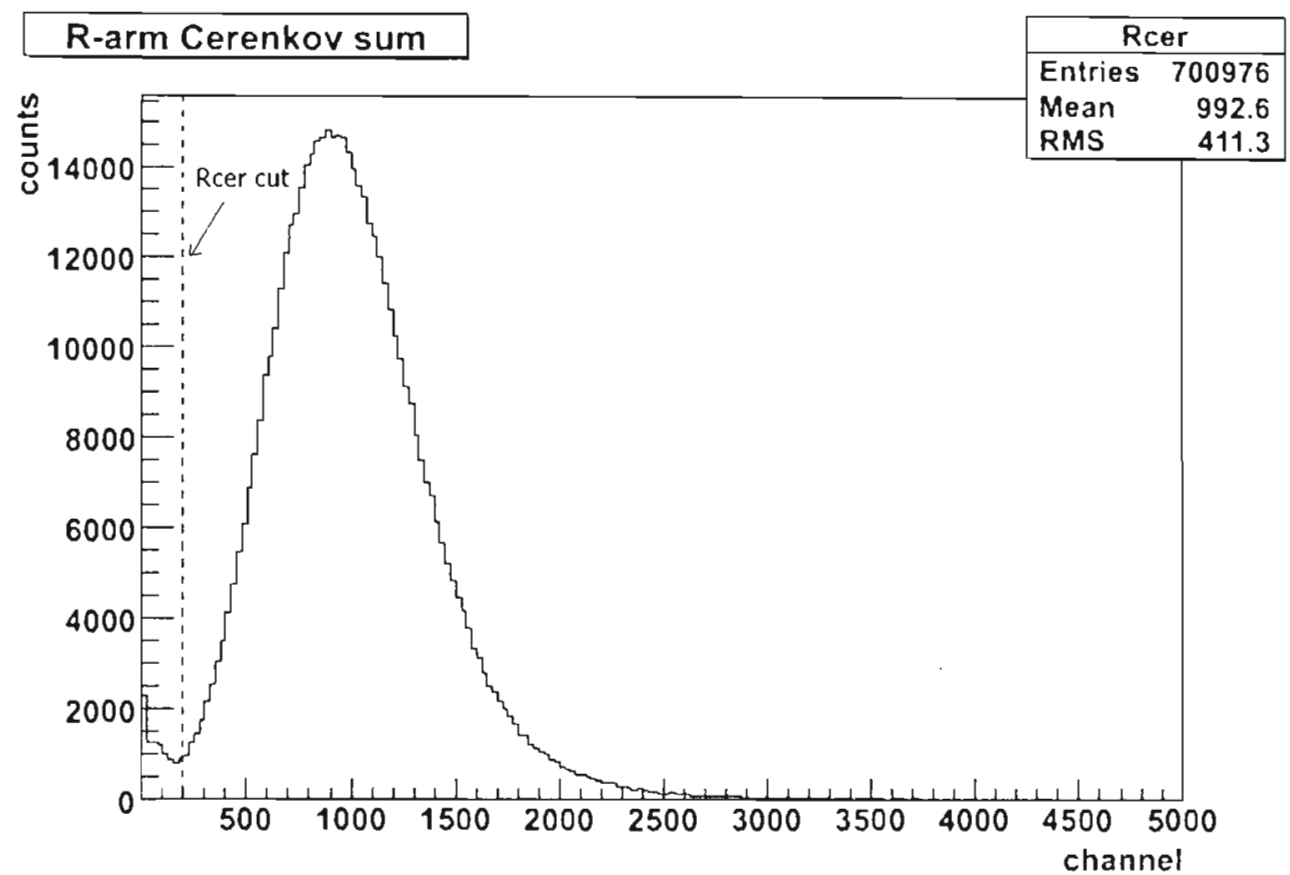

Figure 48: $\mathrm{CO}_{2}$ gas Cherenkov ADC signal in run 2485, showing "Rcer" PID cut according to Equation 45 to discriminate electrons from negative pions.

From Figure 48, the large peak corresponds to electrons while the small peak close to the pedestal corresponds to negative pions $(\pi)$ [68]. Negative pions are more massive than electrons and for this reason they have a lower velocity (at the same momenta) compared to the electrons. Therefore, negative pions produce fewer numbers of photons, which is better shown in Section 4.5. Consequently, the following cut, named "Rcer", was used to discriminate electrons from negative pions in the right arm:

Rcer $>200$ channels.

The next step is to then identify the different particles in the coincidence time of flight (TOF) spectrum. "Coincidence" here means that a particle in the left spectrometer 
was detected in the same time window as a particle in the right spectrometer. For this purpose, two different methods were used. Firstly, only the aerogel PID cuts and $\mathrm{CO}_{2}$ Cherenkov PID (Equation 45) cut were used. Later, aerogel cuts, RICH PID cuts and $\mathrm{CO}_{2}$ Cherenkov PID cut were used. The two different procedures were performed in order to verify that the RICH PID cuts work as well as the aerogel PID cuts. The following Figures from 49 to 57 show the coincidence TOF spectra (or CT spectra) using aerogel PID cuts and $\mathrm{CO}_{2}$ gas Cherenkhov PID cut. The $y$ axis denotes counts while the $x$ axis shows time in nanoseconds. Path length corrections were included in these analyses.

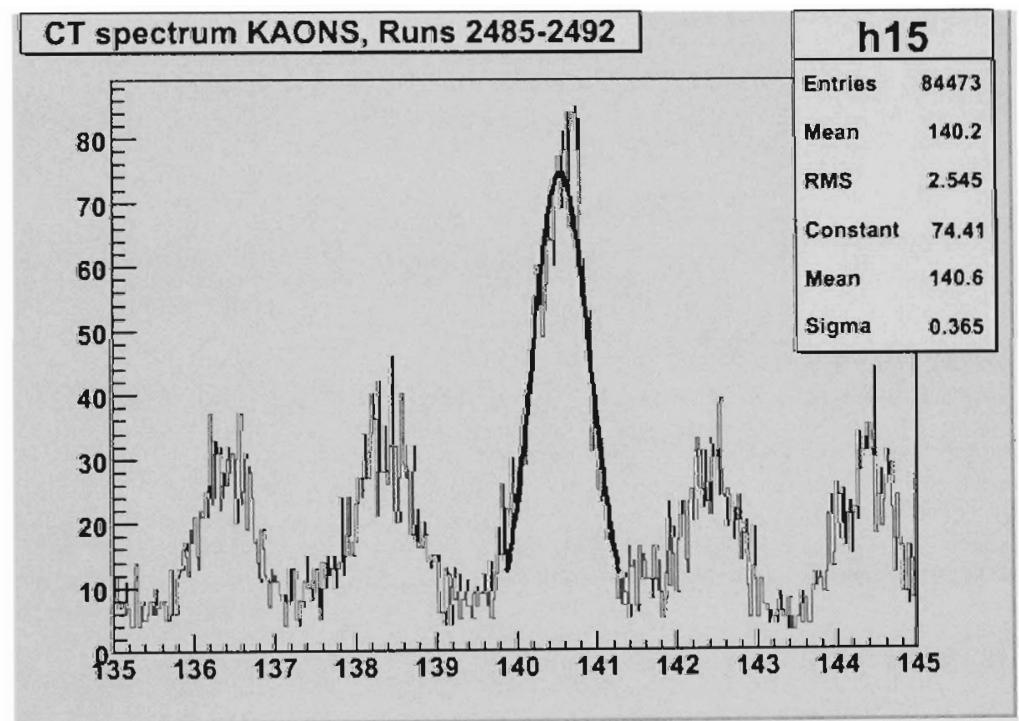

Figure 49: CT spectrum (ns) for particles identified as kaons (KIN_1). 


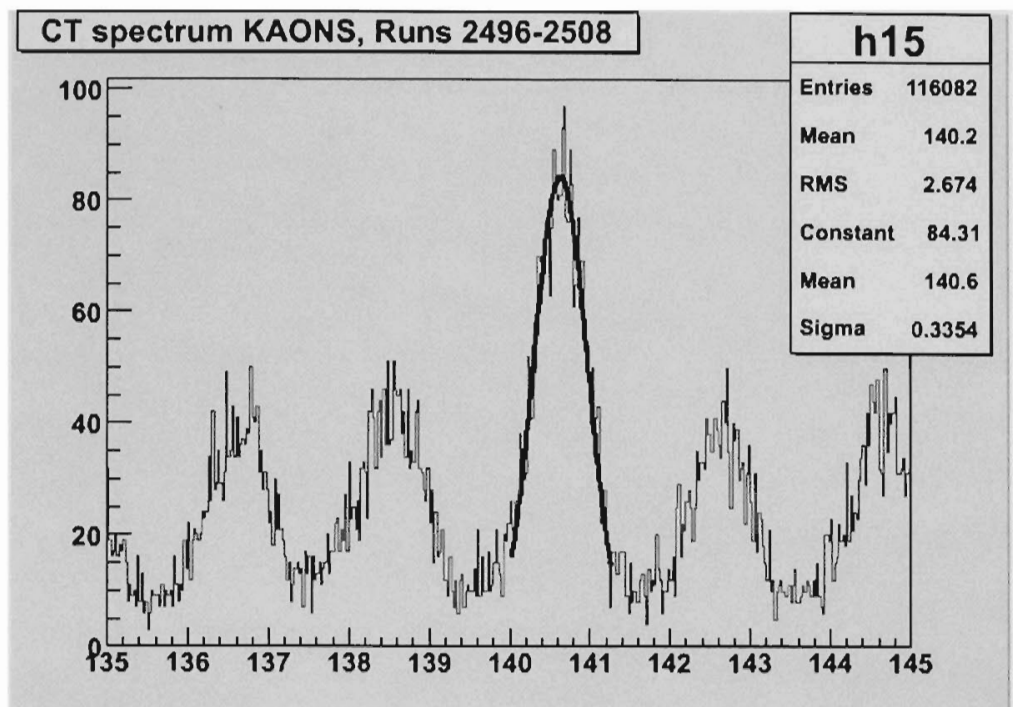

Figure 50: CT spectrum (ns) for particles identified as kaons (KIN_2).

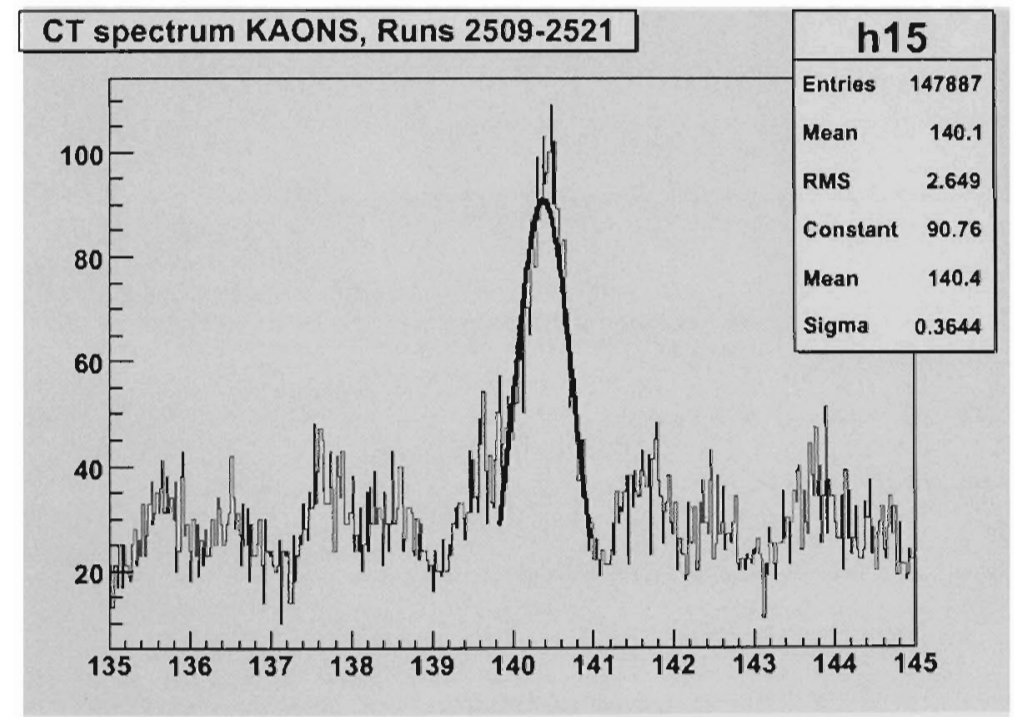

Figure 51: CT spectrum (ns) for particles identified as kaons (KIN_3). 


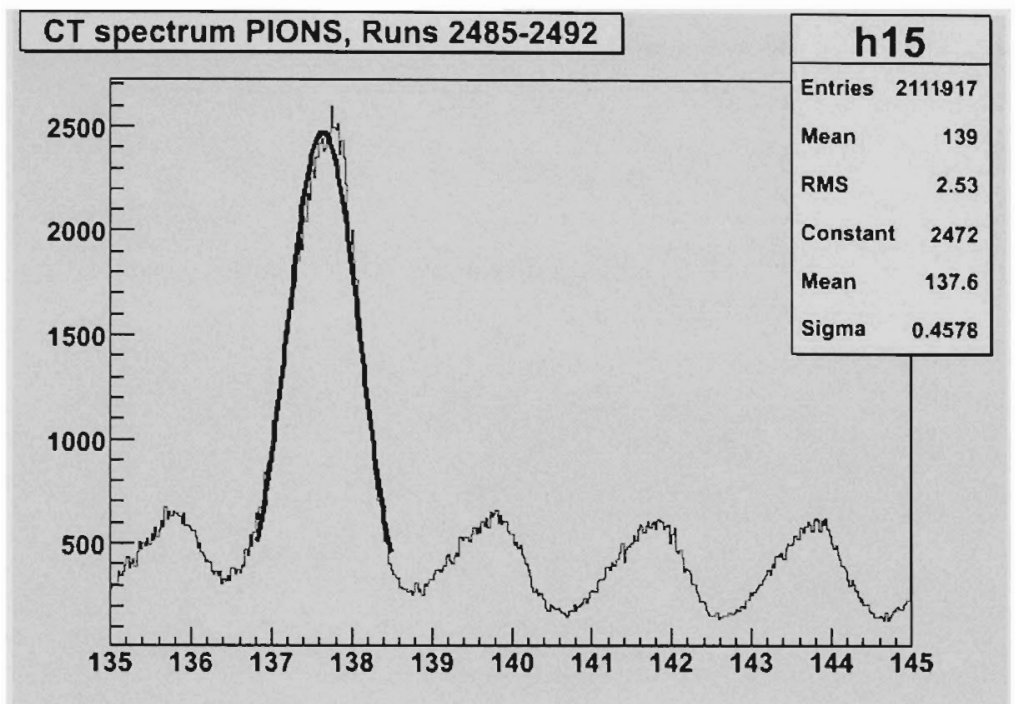

Figure 52: CT spectrum (ns) for particles identified as pions (KIN_1).

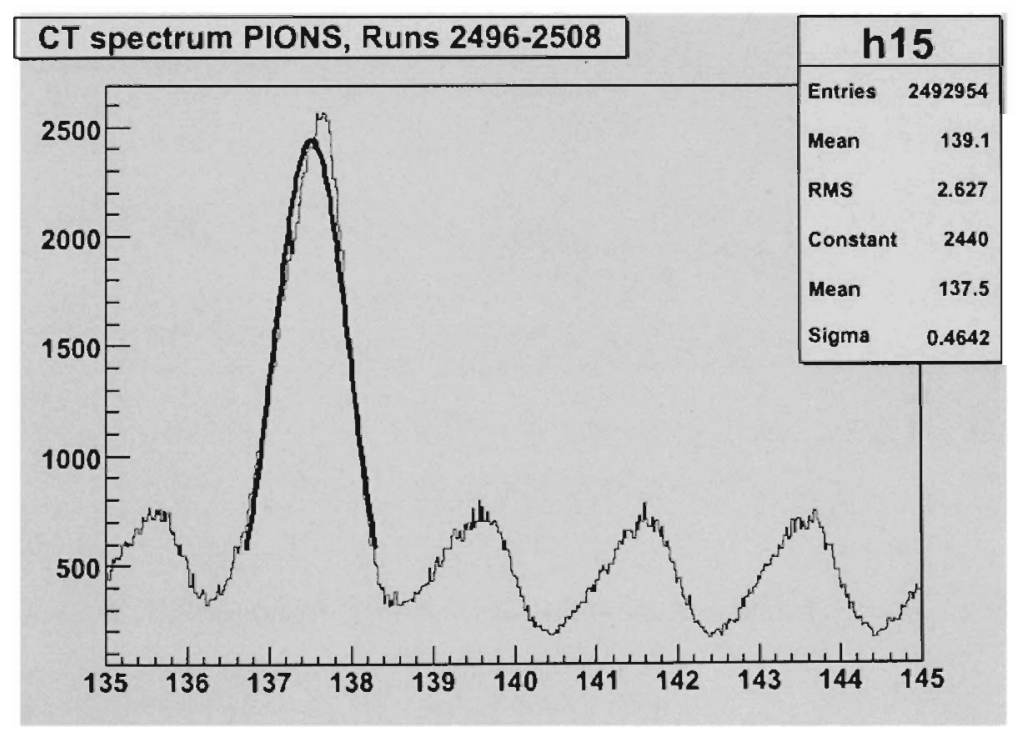

Figure 53: CT spectrum (ns) for particles identified as pions (KIN_2). 


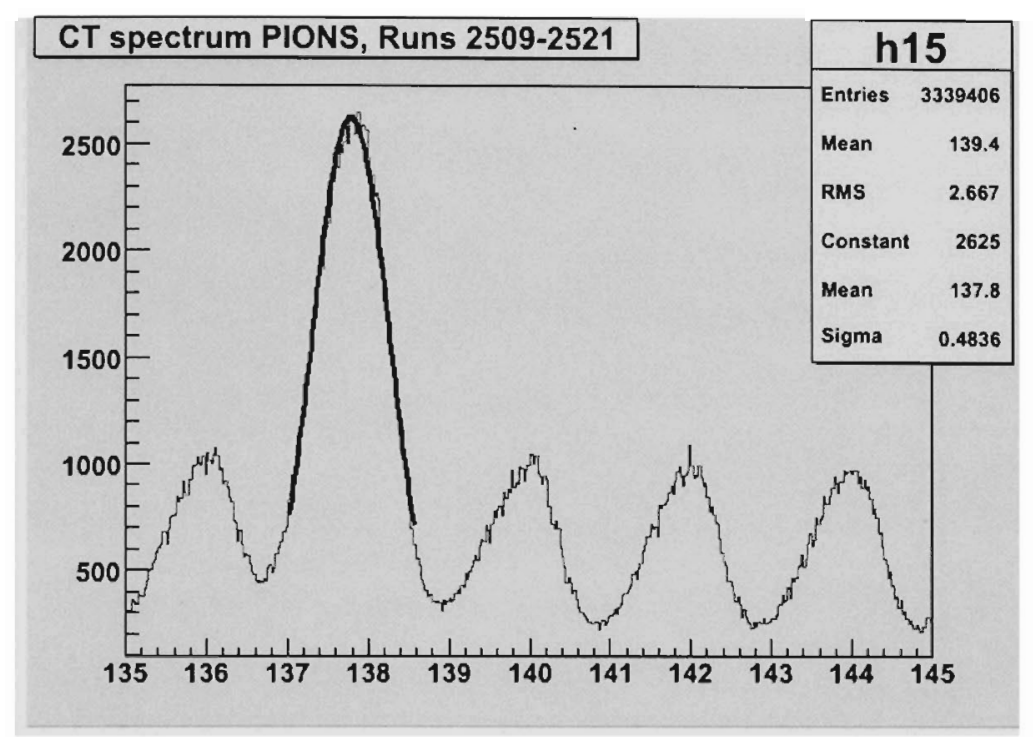

Figure 54: CT spectrum (ns) for particles identified as pions (KIN_3).

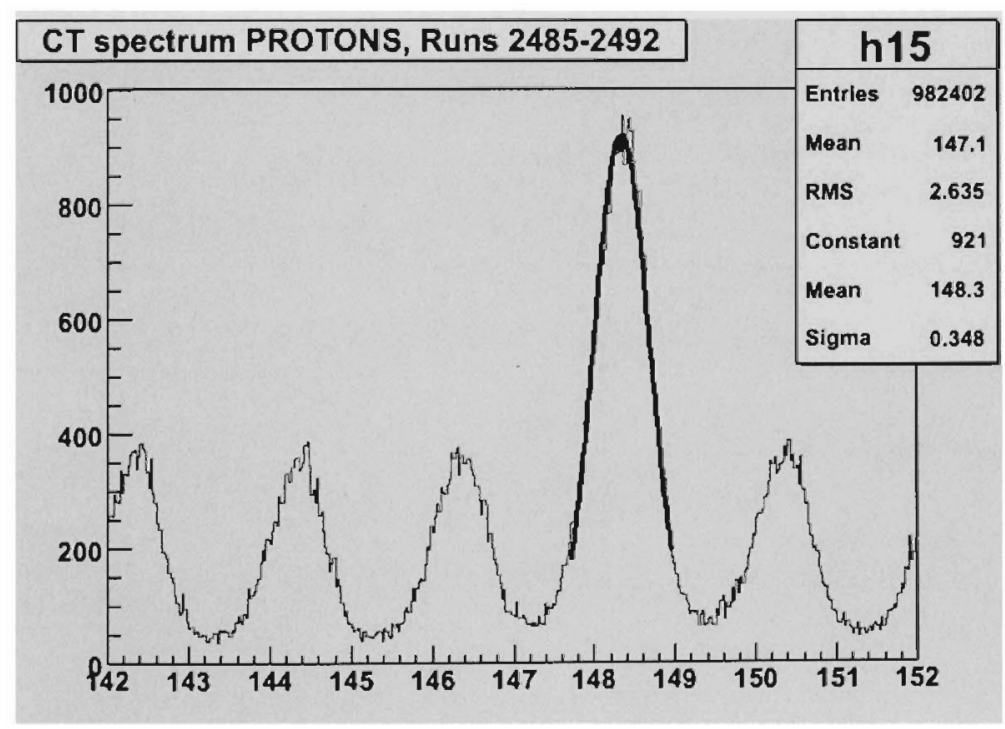

Figure 55: CT spectrum (ns) for particles identified as protons (KIN_1). 


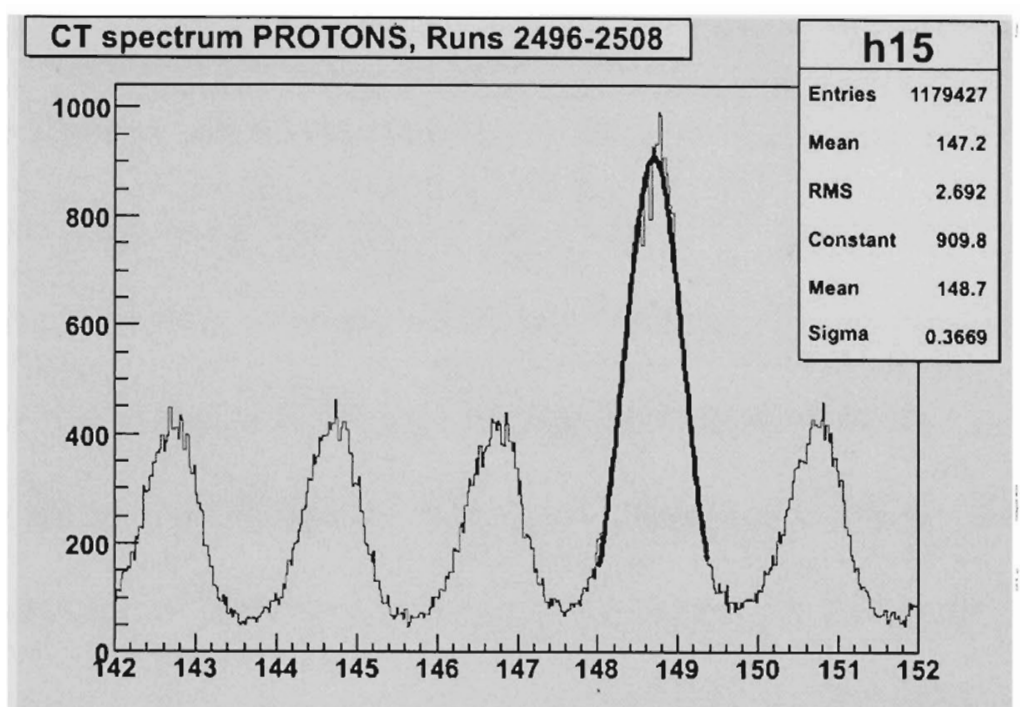

Figure 56: CT spectrum (ns) for particles identified as protons (KIN_2).

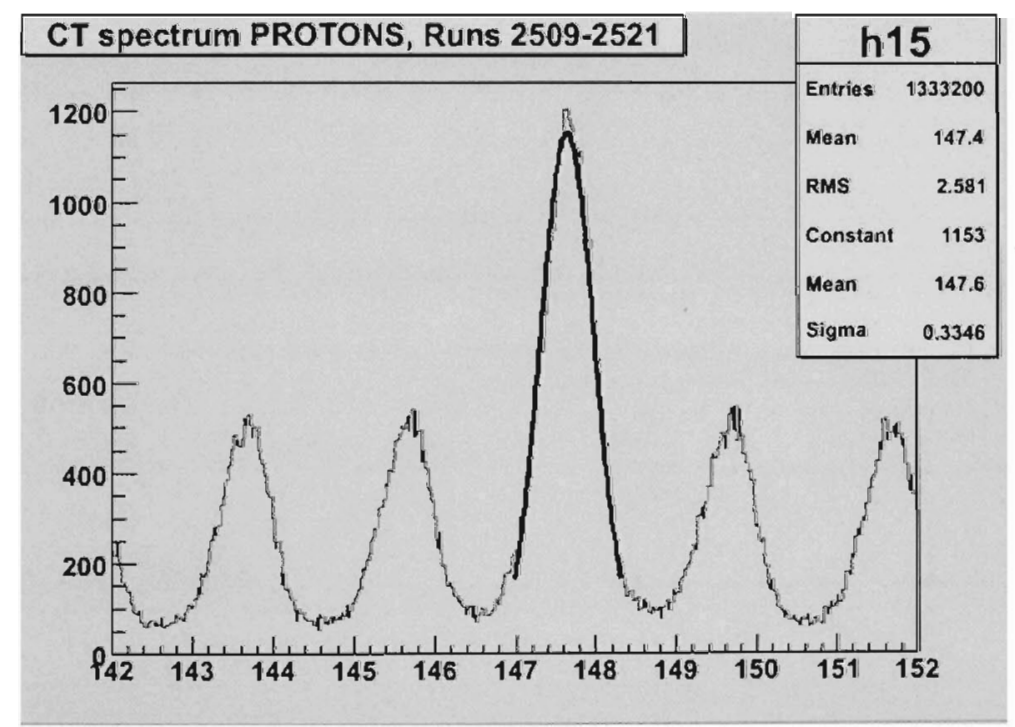

Figure 57: CT spectrum (ns) for particles identified as protons (KIN_3). 
Similar TOF spectra are shown in Appendix D, which include not only the aerogel cuts and $\mathrm{CO}_{2}$ Cherenkov PID cut but also the RICH cuts. In general, it can be seen that both detectors can satisfactorily identify the three different observed particles in the hadron arm. The bump structure of the of the TOF spectra represents accidental coincidences from incident electrons which arriving every $2 \mathrm{~ns}$ at the target. Therefore, a width of \pm ns was assigned to the coincidence time for all particles on the basis of the resolution shown in the $\mathrm{CT}$ spectra. It is also important to remark that the obtained coincidence times for all particles are basically the same if the RICH cuts are used or not. Essentially, the main difference between using only the aerogel cuts and whether the RICH cuts are included or not is that the RICH reduces the statistics significantly. The coincidence times are summarized in Table 19.

\begin{tabular}{cccc}
\hline CTOF & KAONS (ns) & PIONS (ns) & PROTONS (ns) \\
\hline KIN_1 & 140.7 & 137.8 & 148.3 \\
KIN_2 & 140.7 & 137.6 & 148.6 \\
KIN_3 & 140.5 & 137.9 & 147.6 \\
\hline
\end{tabular}

Table 19: Coincidence times for each particle in each kinematics.

\subsection{Missing mass spectra}

The missing mass is the mass that the recoiling undetected third particle in the reaction must have in order to conserve energy and momentum. Sometimes, to make calculations simpler it is convenient to add a constant to the missing mass. In a similar 
way missing energy is defined. The missing mass in the ${ }^{1} \mathrm{H}\left(e, e^{\prime} K^{+}\right) \mathrm{Y}$ reaction of producing hyperons can be reconstructed by using all the cuts described in the previous chapter. Combining energy and momentum conservation yields:

$$
\begin{aligned}
& M_{\Lambda, \Sigma^{0}}^{m i s \sin g}=\sqrt{\left(e-e^{\prime}\right)+p-K} \\
& E_{\Lambda, \Sigma^{0}}^{m i s i n} g=\omega-T_{K}-T_{p}=M_{\Lambda}+M_{K}-M_{p}
\end{aligned}
$$

where $e\left(e^{\prime}\right)$ is the four-momentum of the incident (scattered) electron, $p$ is the fourmomentum of the target proton, $K$ is the four-momentum of the kaon, $M_{p^{+}}$is the rest mass of the proton, $M_{K^{+}}$is the rest mass of the kaon, $M_{\Lambda, \Sigma^{0}}$ is either the rest mass of the Lambda particle or the Sigma particle, $\omega=E_{e}-E_{e^{e}}$ is the energy of the virtual photon, $T_{K}$ is the kinetic energy of the kaon, and $T_{p}$ is the kinetic energy of the proton ( $=0$ in the laboratory frame). $M_{\Lambda, \Sigma^{0}}^{\text {missing }}$ and $E_{\Lambda, \Sigma^{0}}^{\text {missing }}$ are missing mass and energy when a Lambda particle is created or a Sigma particle is created. In principle, it is also possible to create heavier, more excited strange baryons. However, the experimental kinematics limited the possible baryons to the lowest two hyperons.

In Figures 58, 59 and 60, the tails sloping off toward higher missing mass from each of these peaks are caused by the effects of radiative processes. In these processes, the incident electron, scattered electron, or produced kaon loses some energy by radiating a photon. As a result, there is some extra energy and momentum missing from what is measured leading to an apparent increase in the calculated missing mass (tails towards the right of the spectra). 
A cut was placed on the missing mass in order to separate the $\Lambda$ and $\Sigma^{0}$ reaction channels in the analysis (Section 4.8) to select either $\Lambda$ or $\Sigma^{0}$ particles. It can be easily verified that the expected missing energies are $E_{\Lambda}^{m i s \sin g}=670.99 \mathrm{MeV}$ and $E_{\Sigma^{0}}^{m i s \sin 8}=747.89$ $\mathrm{MeV}$. The tails of the missing mass spectrum were included to keep as many hyperons as possible in the analysis statistics. The cuts are shown in Table 20.

\begin{tabular}{l}
\hline $0.6611 \mathrm{GeV}<M_{\Lambda}^{m i s \sin g}<0.7111 \mathrm{GeV}$ \\
\hline $0.6880 \mathrm{GeV}<M_{\Sigma^{0}}^{m i s \sin g}<0.7880 \mathrm{GeV}$
\end{tabular}

Table 20: Applied cuts on missing masses.

The cuts in Table 20 were obtained after examining the missing mass spectra for the different kinematics. Although the cuts seem wide, the Lambda and the Sigma peaks are quite far apart as shown in Figures 58 and 59.

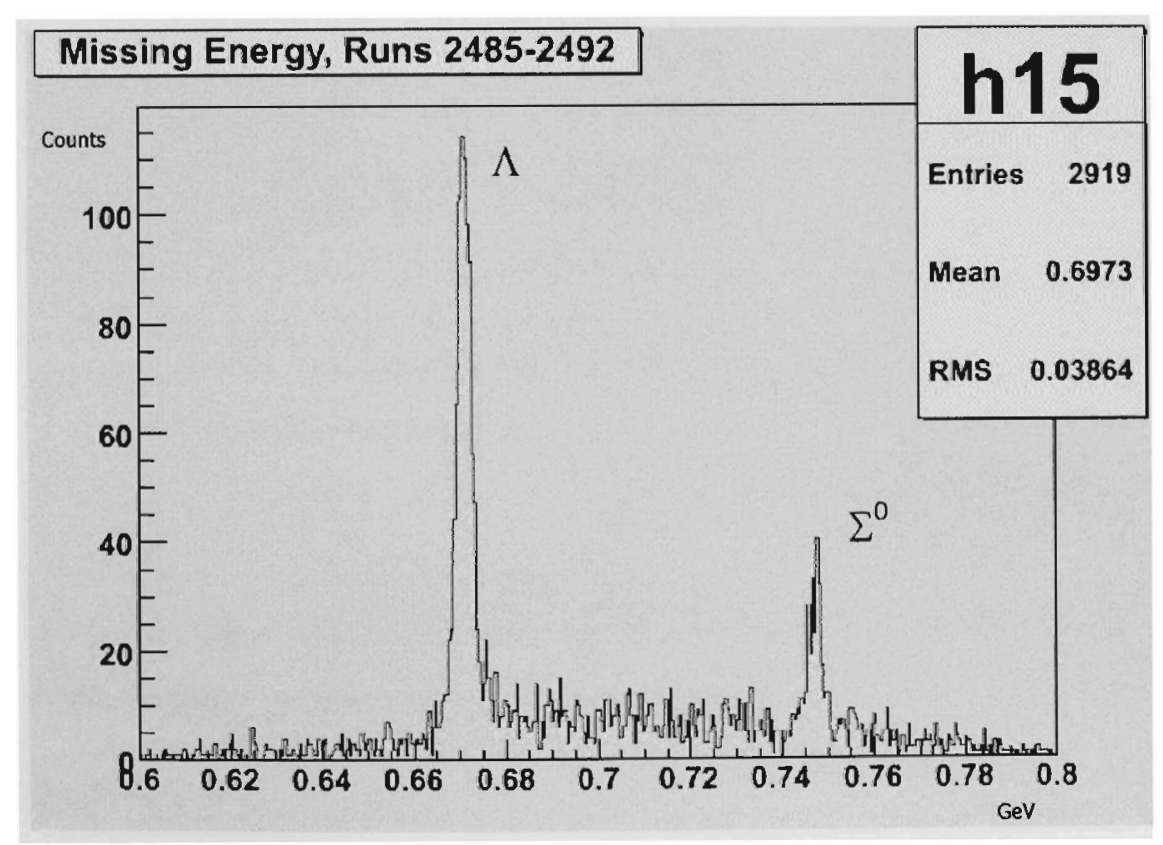

Figure 58: Missing mass spectrum for KIN_1. 


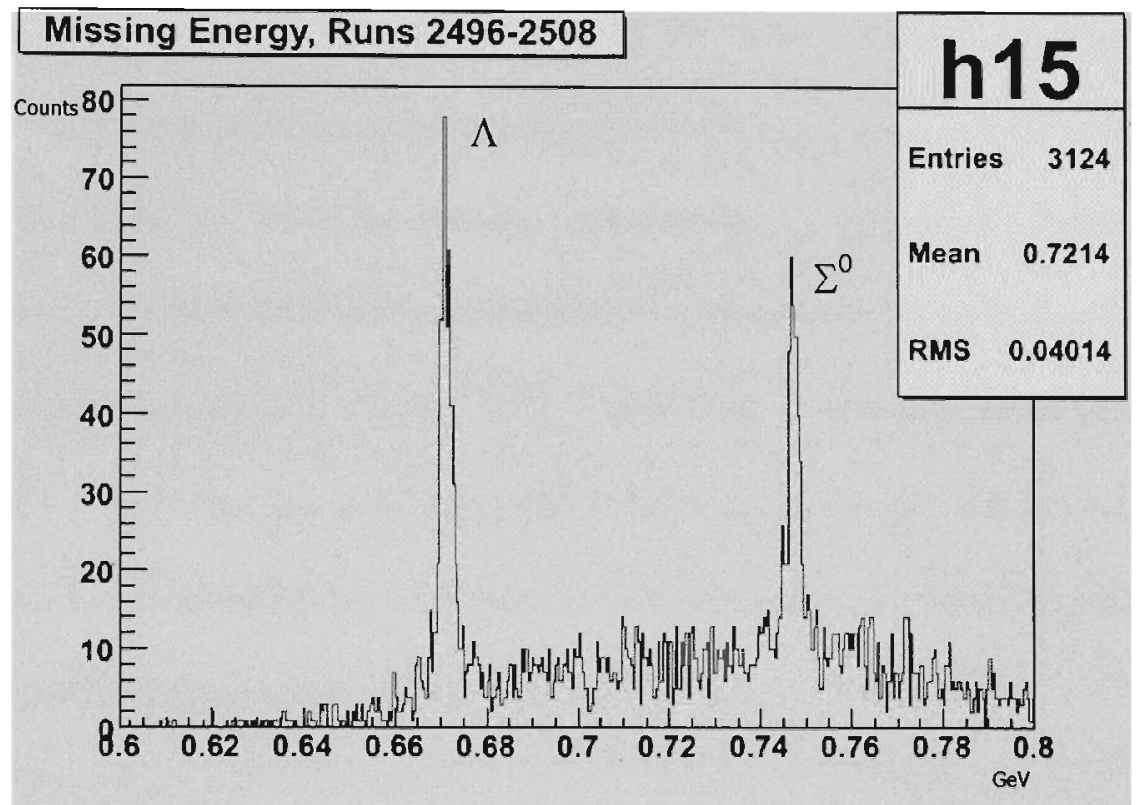

Figure 59: Missing mass spectrum for KIN_2.

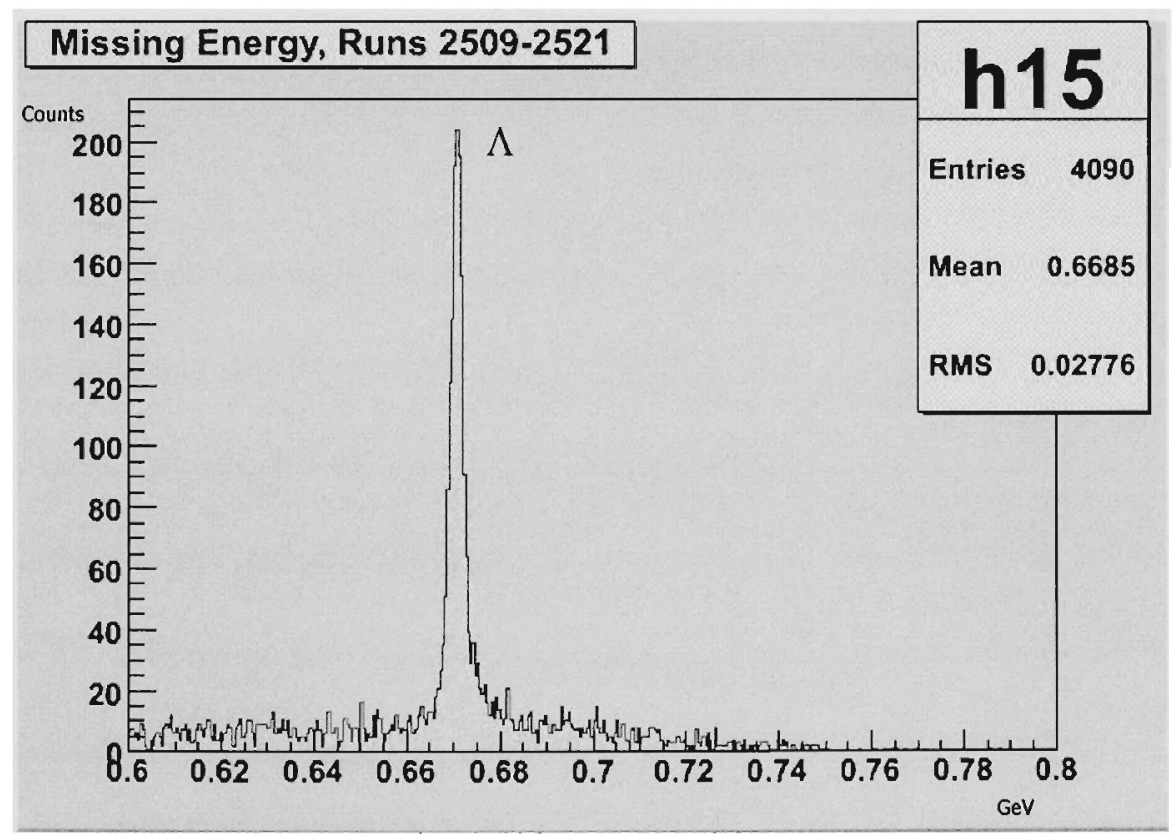

Figure 60: Missing mass spectrum for KIN_3. 
The peaks in the Figures 58, 59 and 60 are quite separated as expected but the observed background still has to be subtracted. The background is primarily caused by kaons and electrons that came from different scattering events and are therefore not true coincidences. The three different momentum settings in the left arm (Table 15) produced three different missing mass spectra. KIN_1 and KIN_2 allow selection either of $\Lambda$ or $\Sigma^{0}$. For KIN_3 only $\Lambda$ s are seen. Regarding the three different kinematics in Table 15 , the produced hyperon selections in Table 20, and the peaks shown in Figures 58, 59 and 60 that five different combinations are obtained as stated in Table 21.

\begin{tabular}{ll}
\hline Analysis & \multicolumn{1}{c}{ Combination } \\
\hline L_KIN_1 & KIN_1 and $\Lambda$ selected \\
L_KIN_2 & KIN_2 and $\Lambda$ selected \\
L_KIN_3 & KIN_3 and $\Lambda$ selected \\
S_KIN_1 & KIN_1 and $\Sigma^{0}$ selected \\
S_KIN_2 & KIN_2 and $\Sigma^{0}$ selected \\
\hline
\end{tabular}

Table 21: Particle/kinematics combinations for analyses.

The missing mass spectra obtained including the RICH PID cuts (see Appendix E) were qualitatively similar to the ones shown in this section that do not contain the RICH PID cuts. The spectra obtained with RICH PID cuts (Table 17), certainly show less background but in the general analysis (Section 4.8) the statistics were lower. Although the spectra get corrected for the RICH efficiency, the inclusion of the RICH causes a larger statistical uncertainty than is desirable. The RICH was a needed detector for PID on the oxygen target to reduce the larger backgrounds and be able to resolve the core excited state peaks, but higher luminosity was needed in return [69]. It will be shown is Section 4.7 that the aerogel PID cuts with no RICH PID are background free and the 
increased statistics provide a reduced statistical uncertainty in the measurement. However, utilizing the $\mathrm{RICH}$ as an extra detector then becomes very useful to determine the aerogel detector analysis (see Section 4.4).

\subsection{Background subtraction}

The missing mass spectra shown in Section 4.2 require background subtraction. The background shape was determined by selecting a region in the CTOF spectra (see Figures 49-57) far from the ccoincident peak. A cut of $160 \mathrm{~ns} \leq \mathrm{CTOF} \leq 180 \mathrm{~ns}$ was used. The region is also sufficiently far from the proton and pion coincidence peaks that the tails of those peaks do not contribute should any of those events leak through the PID cuts. The missing mass analysis is done over this window and then divided by 10 to find the average of the background in a 2 ns window. After that, the background is subtracted from the missing mass spectra, which has a $2 \mathrm{~ns}$ window, resulting in background free spectra as shown in Figures 61, 62 and 63. 


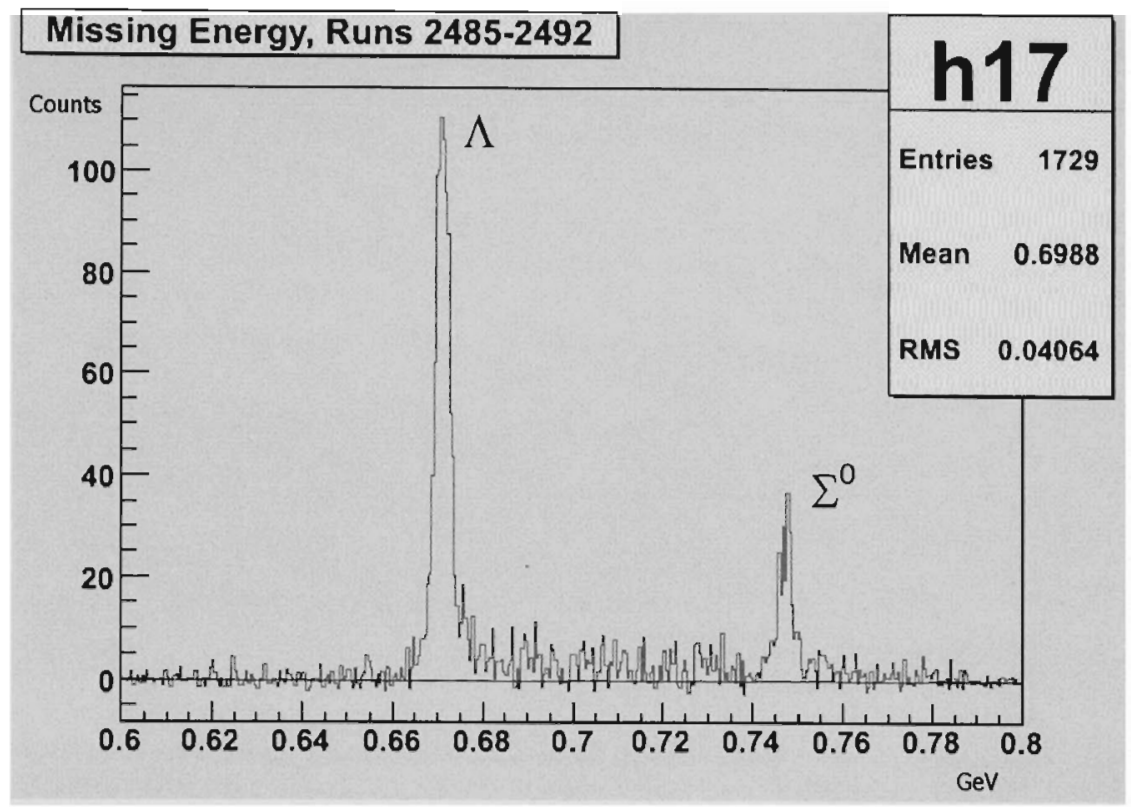

Figure 61: Background substracted spectrum for KIN_1.

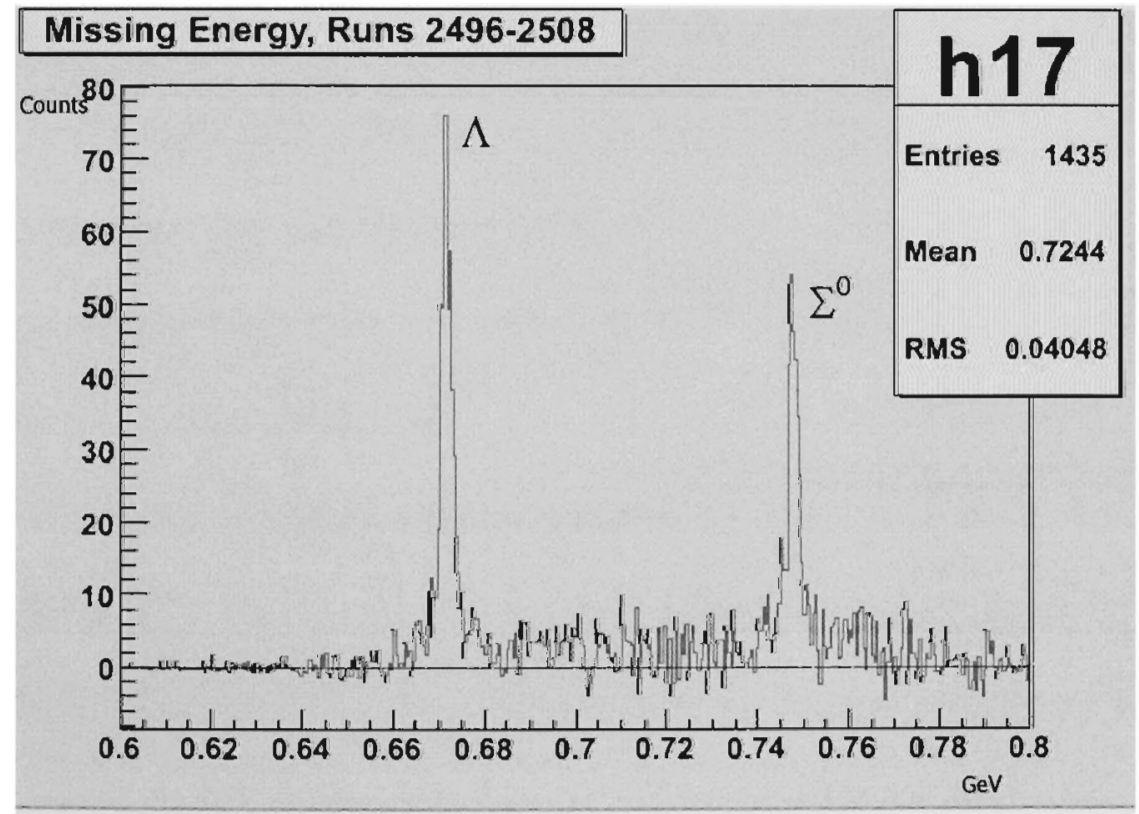

Figure 62: Background substracted spectrum for KIN_2. 


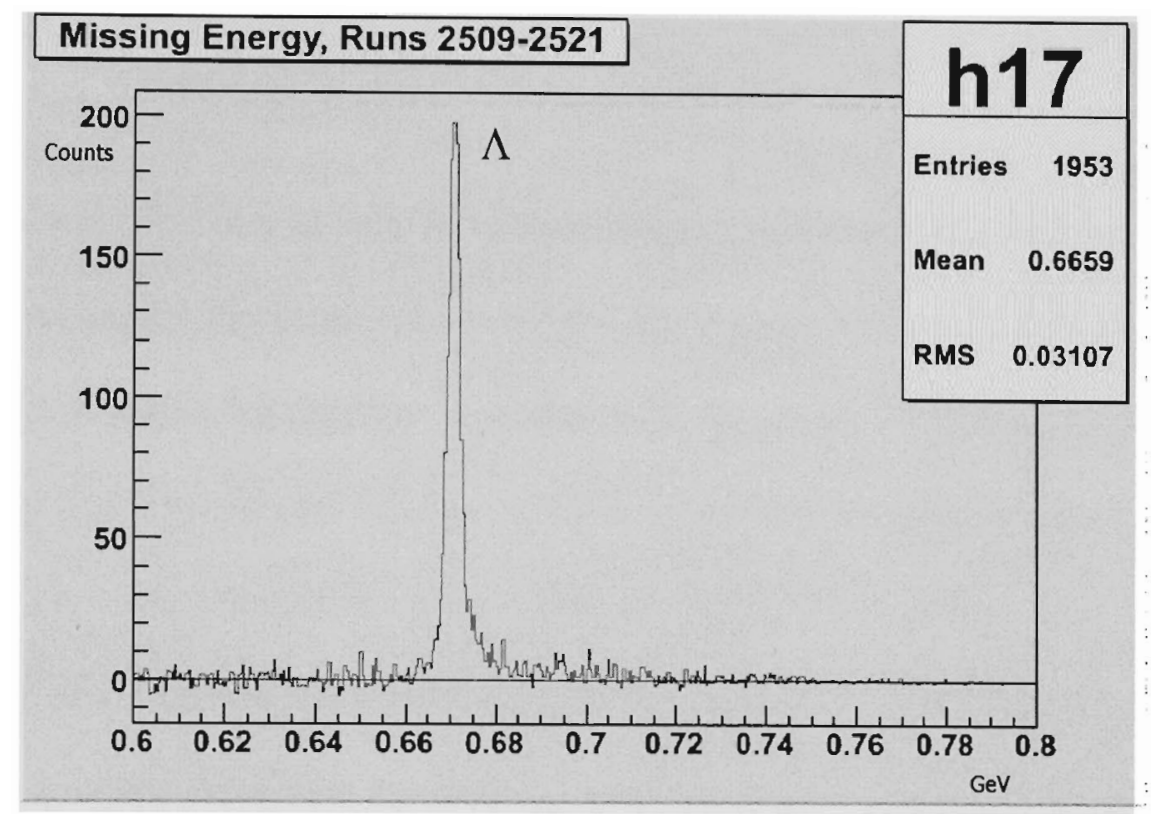

Figure 63: Background substracted spectrum for KIN_3.

It is important to note that there was no need to do "dummy" target subtraction. Although in principle the aluminum target walls can contribute with $\left(e, e^{\prime} K^{+}\right)$events, the yields due to the aluminum walls are so small that they are negligible compared to the out-of-time (or accidental coincidence) background. In addition, the $\Lambda$ and $\Sigma^{0}$ peaks are well enough separated that there was also no need to estimate any overlapping of the $\Sigma^{0}$ peak and the tail of the $\Lambda$ peak [70].

Similarly, the missing mass spectra analysis was repeated including the RICH PID cuts (Table 17). The spectra are similar. However, for reasons explained later in Section 4.7 and 4.8 the RICH was not used to measure the cross section as a result of the low efficiency of the $\mathrm{RICH}$ (about $75 \%$ to $85 \%$ ) for the $\mathrm{LH}_{2}$ cryotarget. See at the end of Section 4.4 and Appendix G. 
It is also necessary to determine the efficiency of the aerogel detectors A1 and A2 to be able to extract the cross section for the elementary reaction. These two detectors provided the primary PID for these measurements. However, it is important to be precise when describing how aerogel detector A1 was used, since it must not fire (i.e., produce Cherenkov photons) when a kaon passes through. Therefore, in case of A1 we refer to the inefficiency of the detector (see Section 2.6.5). In order words, the inefficiency of A1 will tell us how good the detector is in not firing when kaons pass through it. Physically, when kaons traverse the detector they might knock out electrons, which would be energetic enough to fire the aerogel.

The analysis of the aerogel detectors is not totally trivial. They are diffusion boxes, in which the Cherenkov light produced may scatter several times before being detected, and because the experiment is performed with three different momenta and two different hyperons being selected, the analysis of the aerogel detectors efficiencies must be considered separately according to the different combination of kinematics and particle (hyperon) selection (see Table 21). These different kinematics results in different kaon momenta or equivalently velocities. Having different momenta set and selecting different particles effectively selects different kaon momenta in the aerogel diffusion boxes. Therefore, the efficiency of the aerogel boxes will depend not only on the momentum set in the hadron arm but also on the hyperon selected. For this reason, five different results for the aerogel efficiencies are expected, each averaged over the range of kaon momenta used. 
The analysis of either A1 or A2 relies on the use of other detectors to select a clean sample of kaons. The RICH detector was used in this case along with the second aerogel to select a clean sample of kaons using the cuts shown in the RICH analysis as detailed in Table 17. In order words, if A1 is being analyzed a tight cut is applied to A2 or vice versa and always with the RICH cuts for kaons. The tight cuts in A1 and A2 applied areshown in Table 22.

\begin{tabular}{cc}
\hline Detector & $\begin{array}{c}\text { Aerogel cuts for efficiency analysis. } \\
\text { (channels) }\end{array}$ \\
\hline A1 & $100<$ A $2<300$ \\
A2 & A $1<10$ \\
\hline
\end{tabular}

Table 22: Aerogel tight cuts used for efficiency analysis.

On top of the mentioned cuts in the previous lines, background subtraction is applied (see Section 4.3) and additional tight cuts in the missing mass. Basically, these two tight cuts are narrow regions placed around the hyperon mass peaks in the missing mass spectra. The cuts are shown in Table 23.

\begin{tabular}{cc}
\hline Hyperon selected & $\begin{array}{c}\text { Missing mass cut for Aerogel analysis } \\
(\mathrm{GeV})\end{array}$ \\
\hline$\Lambda$ & $0.6682<M_{\Lambda}^{\text {missing }}<0.6736$ \\
$\Sigma^{0}$ & $0.7462<M_{\Sigma^{0}}^{\text {missing }}<0.7488$ \\
\hline
\end{tabular}

Table 23: Hyperon selection tight cut for the aerogel analysis. 
Tables 24-33 and Figures 64-73 are a sequence showing the results for both aerogel detector analyses with the five possible combinations (Table 21).

\begin{tabular}{|c|c|c|c|c|c|}
\hline KAONS & & A1sum & $\begin{array}{c}\mathrm{A} 2>100 \\
\text { and } \\
\mathrm{A} 2<300\end{array}$ & Inefficiency & \\
\hline \multirow{3}{*}{$\begin{array}{c}\mathrm{RICH}+\mathrm{A} 2: \\
\text { A1 CUT } \\
\quad(<)\end{array}$} & 569 & 79 & 561 & & \\
\hline & $\mathrm{RICH}+\mathrm{AERO}$ & counts & & & \\
\hline & TOTAL & $\begin{array}{l}\text { ACCIDENTAL } \\
\left(*^{*} 10\right)\end{array}$ & TRUE & normalized & uncertainty \\
\hline-10 & 0 & 0 & 0 & 0.0000 & 0.0000 \\
\hline 0 & 0 & 0 & 0 & 0.0000 & 0.0000 \\
\hline 5 & 516 & 65 & 509 & 0.9073 & 0.0122 \\
\hline 10 & 518 & 65 & 511 & 0.9109 & 0.0120 \\
\hline 25 & 521 & 66 & 514 & 0.9162 & 0.0117 \\
\hline 50 & 528 & 66 & 521 & 0.9287 & 0.0109 \\
\hline 75 & 535 & 68 & 528 & 0.9412 & 0.0099 \\
\hline 100 & 544 & 69 & 537 & 0.9572 & 0.0085 \\
\hline 125 & 553 & 74 & 545 & 0.9715 & 0.0070 \\
\hline 150 & 559 & 74 & 551 & 0.9822 & 0.0056 \\
\hline 175 & 559 & 75 & 551 & 0.9822 & 0.0056 \\
\hline 200 & 559 & 75 & 551 & 0.9822 & 0.0056 \\
\hline
\end{tabular}

Table 24: A1 inefficiency dependence on Al cut for $\mathrm{L}_{-} \mathrm{KIN} \_1$.

A1 inEFFICIENCY FOR RUN 2485-2492 WITH RICH (Lambda)

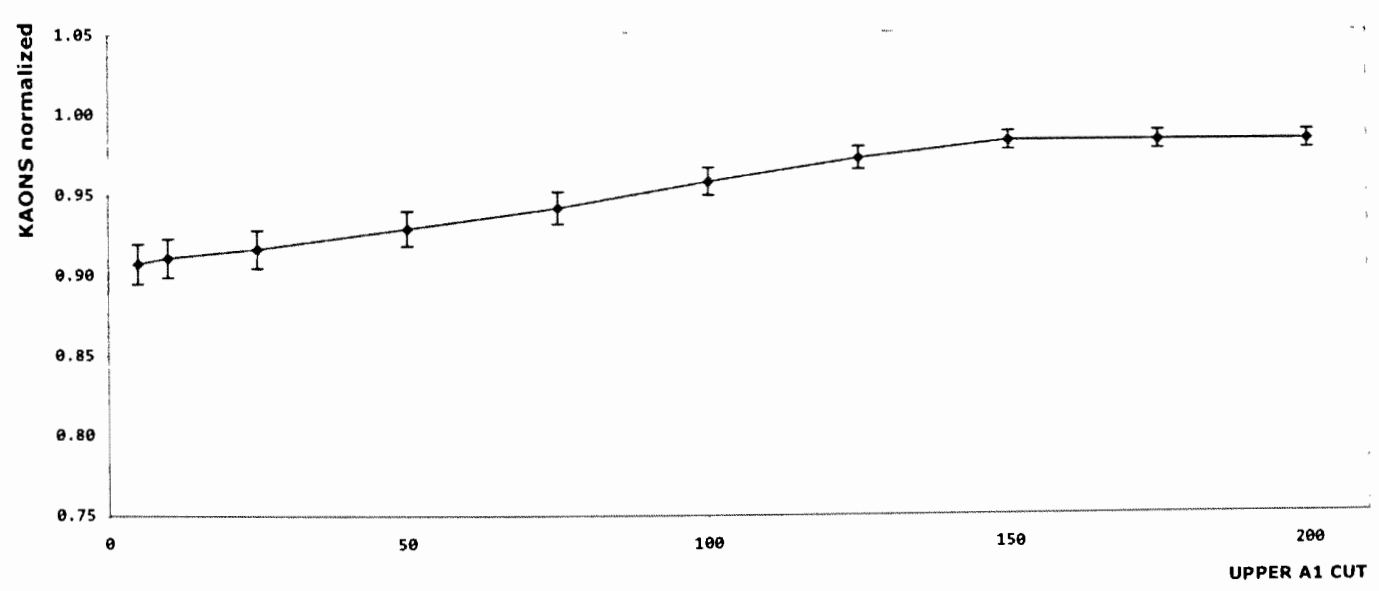

Figure 64: A1 inefficiency dependence on A1 cut for L_KIN_1. 


\begin{tabular}{|c|c|c|c|c|c|}
\hline KAONS & & Alsum & $\begin{array}{c}\mathrm{A} 2>100 \\
\text { and } \\
\mathrm{A} 2<300\end{array}$ & & \\
\hline \multirow{3}{*}{$\begin{array}{c}\mathrm{RICH}+\mathrm{A} 2: \\
\text { A1 CUT } \\
(<)\end{array}$} & 305 & 55 & 299 & & \\
\hline & $\mathrm{RICH}+\mathrm{AERO}$ & counts & & inefficiency & \\
\hline & TOTAL & $\begin{array}{l}\text { ACCIDENTAL } \\
\left({ }^{*} 10\right)\end{array}$ & TRUE & normalized & uncertainty \\
\hline-10 & 0 & 0 & 0 & 0 & 0.0000 \\
\hline 0 & 0 & 0 & 0 & 0 & 0.0000 \\
\hline 5 & 270 & 50 & 265 & 0.8863 & 0.0184 \\
\hline 10 & 275 & 50 & 270 & 0.9030 & 0.0171 \\
\hline 25 & 277 & 51 & 271 & 0.9064 & 0.0168 \\
\hline 50 & 280 & 51 & 274 & 0.9164 & 0.0160 \\
\hline 75 & 285 & 52 & 279 & 0.9331 & 0.0144 \\
\hline 100 & 292 & 52 & 286 & 0.9565 & 0.0118 \\
\hline 125 & 299 & 53 & 293 & 0.9799 & 0.0081 \\
\hline 150 & 302 & 55 & 296 & 0.9900 & 0.0058 \\
\hline 175 & 303 & 55 & 297 & 0.9933 & 0.0047 \\
\hline 200 & 303 & 55 & 297 & 0.9933 & 0.0047 \\
\hline
\end{tabular}

Table 25: A1 inefficiency dependence on A1 cut for L_KIN_2.

A1 inEFFICIENCY FOR RUN 2496-2508 WITH RICH (Lambda)

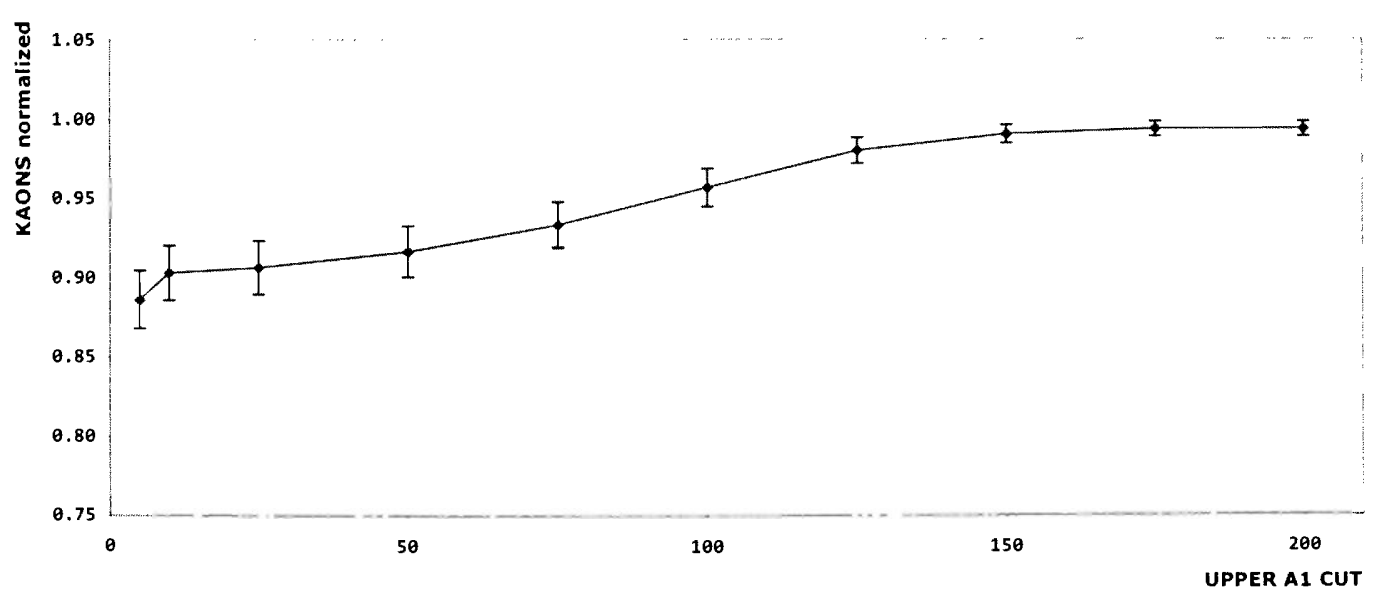

Figure 65: A1 inefficiency dependence on A1 cut for L_KIN_2. 


\begin{tabular}{cccccc}
\hline \multicolumn{5}{c}{ A2>100 } \\
KAONS & \multicolumn{5}{c}{$\begin{array}{c}\text { and } \\
\text { A2<300 }\end{array}$} \\
\hline $\begin{array}{c}\text { RICH+A2: } \\
\text { A1 CUT } \\
(<)\end{array}$ & 406 & RICH+AERO & $\begin{array}{c}\text { counts } \\
\text { ACCIDENTAL }\end{array}$ & 399 & inefficiency \\
& TOTAL & $\left({ }^{*} 10\right)$ & TRUE & normalized & uncertainty \\
-10 & 0 & 0 & 0 & 0 & 0.0000 \\
0 & 0 & 0 & 0 & 0 & 0.0000 \\
5 & 354 & 55 & 348 & 0.8722 & 0.0167 \\
10 & 362 & 55 & 356 & 0.8922 & 0.0155 \\
25 & 364 & 56 & 358 & 0.8972 & 0.0152 \\
50 & 372 & 57 & 366 & 0.9173 & 0.0138 \\
75 & 378 & 58 & 372 & 0.9323 & 0.0126 \\
100 & 390 & 58 & 384 & 0.9624 & 0.0095 \\
125 & 399 & 60 & 393 & 0.9850 & 0.0061 \\
150 & 400 & 60 & 394 & 0.9875 & 0.0056 \\
175 & 401 & 60 & 395 & 0.9900 & 0.0050 \\
200 & 401 & 61 & 394 & 0.9875 & 0.0056 \\
\hline
\end{tabular}

Table 26: A1 inefficiency dependence on $\mathrm{A} 1$ cut for $\mathrm{L}_{-} \mathrm{KIN} \_3$.

A1 inEFFICIENCY FOR RUN 2509-2521 WITH RICH (Lambda)

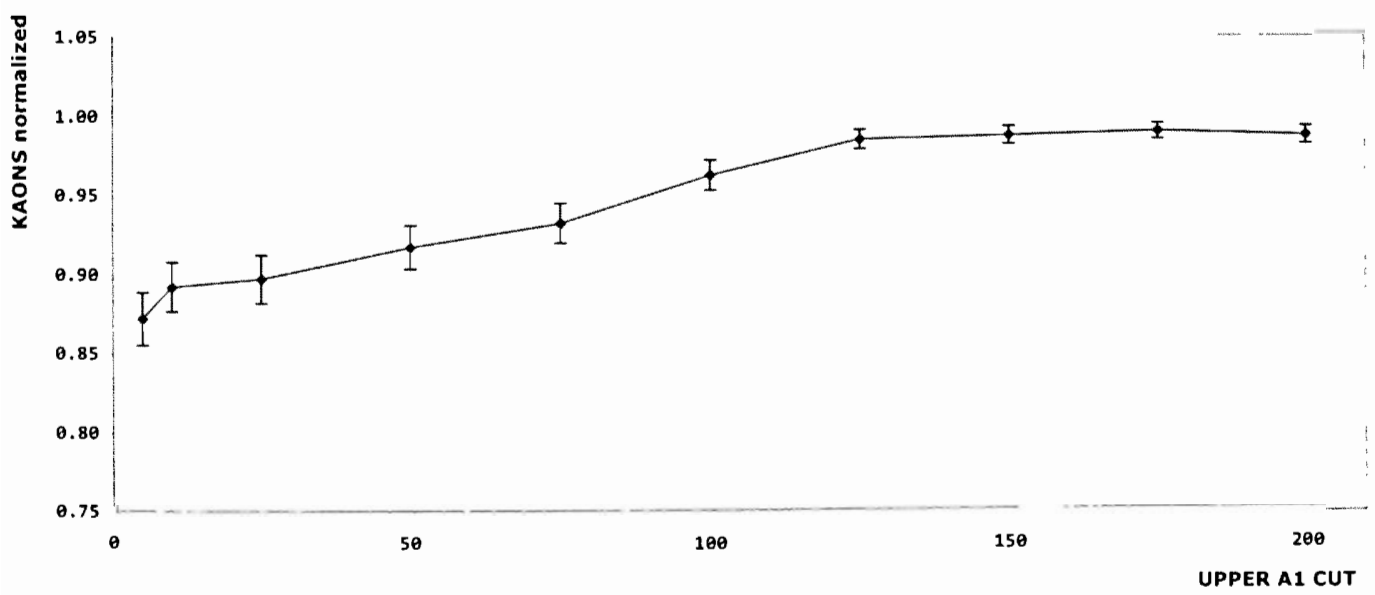

Figure 66: A1 inefficiency dependence on Al cut for L_KIN_3. 


\begin{tabular}{|c|c|c|c|c|c|}
\hline KAONS & & A2sum & $\mathrm{A} 1<10$ & & \\
\hline \multirow{3}{*}{$\begin{array}{c}\mathrm{RICH}+\mathrm{A} 1: \\
\mathrm{A} 2 \mathrm{CUT} \\
(>)\end{array}$} & 985 & 188 & 966 & & \\
\hline & $\mathrm{RICH}+\mathrm{AERO}$ & counts & & efficiency & \\
\hline & TOTAL & $\begin{array}{l}\text { ACCIDENTAL } \\
(* 10)\end{array}$ & TRUE & normalized & uncertainty \\
\hline-10 & 985 & 188 & 966 & 1.0000 & 0.0000 \\
\hline 0 & 979 & 163 & 962 & 0.9959 & 0.0021 \\
\hline 10 & 979 & 160 & 963 & 0.9969 & 0.0018 \\
\hline 20 & 978 & 159 & 962 & 0.9959 & 0.0021 \\
\hline 100 & 911 & 145 & 896 & 0.9275 & 0.0083 \\
\hline 200 & 682 & 112 & 670 & 0.6936 & 0.0148 \\
\hline 300 & 393 & 80 & 385 & 0.3986 & 0.0158 \\
\hline 400 & 216 & 54 & 210 & 0.2174 & 0.0133 \\
\hline 500 & 103 & 30 & 100 & 0.1035 & 0.0098 \\
\hline 600 & 49 & 17 & 47 & 0.0487 & 0.0069 \\
\hline 700 & 38 & 11 & 36 & 0.0373 & 0.0061 \\
\hline 800 & 31 & 7 & 30 & 0.0311 & 0.0056 \\
\hline 900 & 26 & 7 & 25 & 0.0259 & 0.0051 \\
\hline 1000 & 19 & 6 & 18 & 0.0186 & 0.0044 \\
\hline
\end{tabular}

Table 27: A2 efficiency dependence on A2 cut for L_KIN_1.

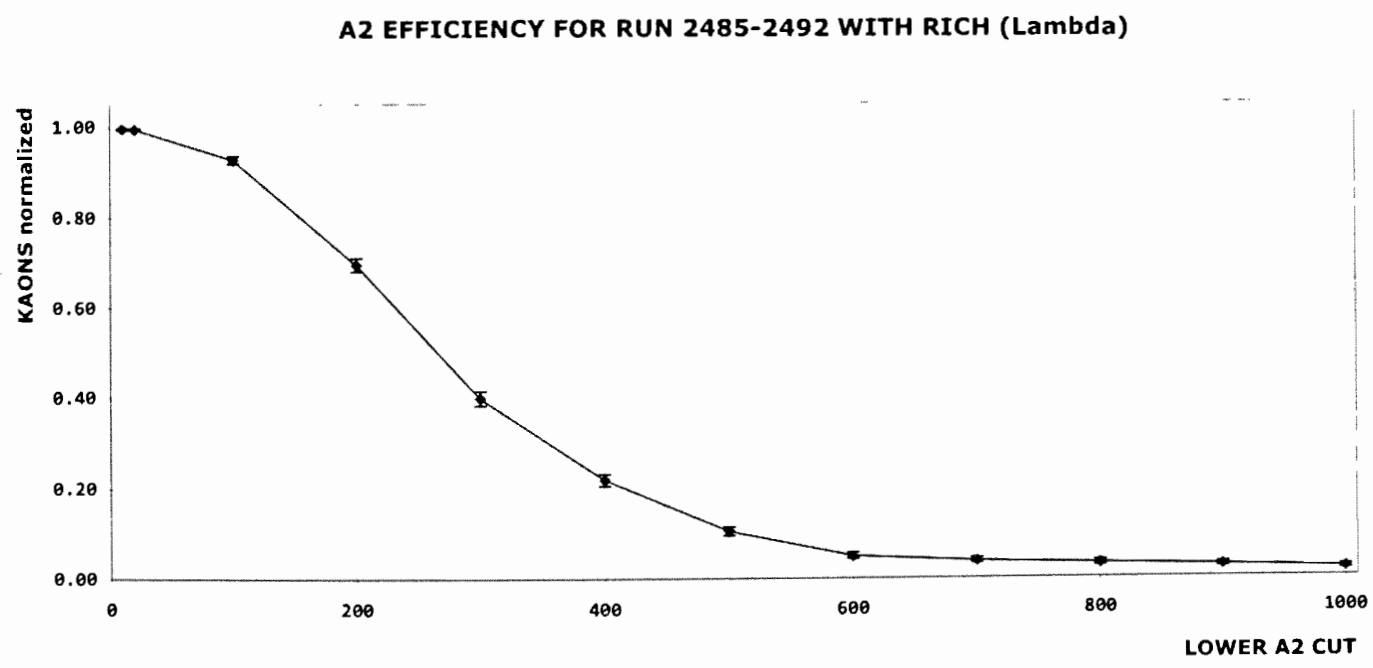

Figure 67: A2 efficiency dependence on A2 cut for L_KIN_1. 


\begin{tabular}{|c|c|c|c|c|c|}
\hline KAONS & & A2sum & $\mathrm{A} 1<10$ & & \\
\hline \multirow{4}{*}{$\begin{array}{l}\mathrm{RICH}+\mathrm{A} 1: \\
\mathrm{A} 2 \mathrm{CUT} \\
(>)\end{array}$} & 484 & 108 & 473 & & \\
\hline & & & & & \\
\hline & $\mathrm{RICH}+\mathrm{AERO}$ & & & efficiency & \\
\hline & TOTAL & $\begin{array}{l}\text { ACCIDENTAL } \\
(* 10)\end{array}$ & TRUE & normalized & uncertainty \\
\hline-10 & 484 & 108 & 473 & 1.0000 & 0.0000 \\
\hline 0 & 481 & 103 & 470 & 0.9937 & 0.0037 \\
\hline 10 & 481 & 102 & 470 & 0.9937 & 0.0037 \\
\hline 20 & 481 & 101 & 470 & 0.9937 & 0.0037 \\
\hline 100 & 448 & 97 & 438 & 0.9260 & 0.0120 \\
\hline 200 & 315 & 73 & 307 & 0.6490 & 0.0219 \\
\hline 300 & 173 & 47 & 168 & 0.3552 & 0.0220 \\
\hline 400 & 80 & 29 & 77 & 0.1628 & 0.0170 \\
\hline 500 & 38 & 20 & 36 & 0.0761 & 0.0122 \\
\hline 600 & 22 & 16 & 20 & 0.0423 & 0.0093 \\
\hline 700 & 12 & 12 & 10 & 0.0211 & 0.0066 \\
\hline 800 & 10 & 9 & 9 & 0.0190 & 0.0063 \\
\hline 900 & 10 & 1 & 9 & 0.0190 & 0.0063 \\
\hline 1000 & 7 & 0 & 7 & 0.0148 & 0.0056 \\
\hline
\end{tabular}

Table 28: A2 efficiency dependence on A2 cut for L_KIN_2.

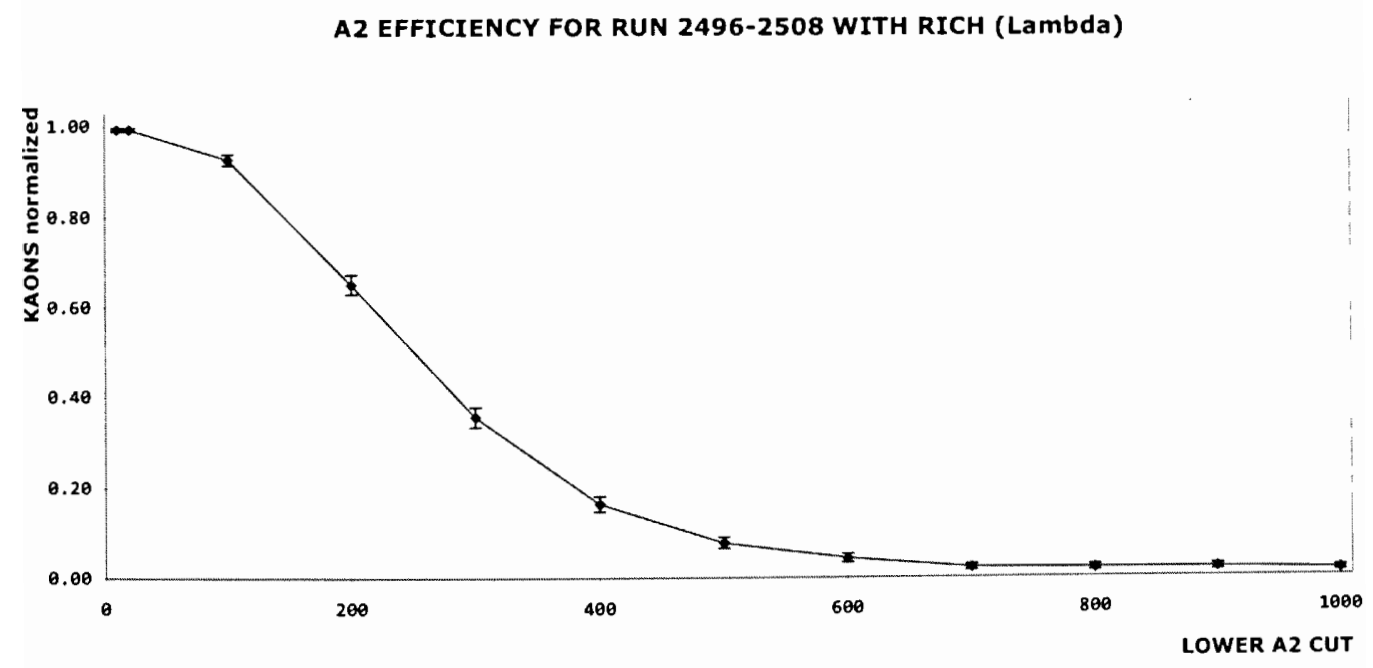

Figure 68: A2 efficiency dependence on A2 cut for L_KIN_2. 


\begin{tabular}{|c|c|c|c|c|c|}
\hline KAONS & & A2sum & $\mathrm{A} 1<10$ & & \\
\hline \multirow{3}{*}{$\begin{array}{l}\mathrm{RICH}+\mathrm{A} 1: \\
\mathrm{A} 2 \mathrm{CUT} \\
(>)\end{array}$} & 809 & 159 & 793 & & \\
\hline & $\mathrm{RICH}+\mathrm{AERO}$ & counts & & & \\
\hline & TOTAL & $\begin{array}{l}\text { ACCIDENTAL } \\
\left({ }^{*} 10\right)\end{array}$ & TRUE & normalized & uncertainty \\
\hline-10 & 809 & 159 & 793 & 1.0000 & 0.0000 \\
\hline 0 & 806 & 136 & 792 & 0.9987 & 0.0013 \\
\hline 10 & 805 & 134 & 791 & 0.9975 & 0.0018 \\
\hline 20 & 802 & 133 & 788 & 0.9937 & 0.0028 \\
\hline 100 & 751 & 123 & 738 & 0.9306 & 0.0090 \\
\hline 200 & 614 & 98 & 604 & 0.7617 & 0.0151 \\
\hline 300 & 389 & 68 & 382 & 0.4817 & 0.0177 \\
\hline 400 & 207 & 46 & 202 & 0.2547 & 0.0155 \\
\hline 500 & 105 & 23 & 102 & 0.1286 & 0.0119 \\
\hline 600 & 51 & 16 & 49 & 0.0618 & 0.0086 \\
\hline 700 & 25 & 9 & 24 & 0.0303 & 0.0061 \\
\hline 800 & 18 & 6 & 17 & 0.0214 & 0.0051 \\
\hline 900 & 14 & 5 & 13 & 0.0164 & 0.0045 \\
\hline 1000 & 12 & 4 & 11 & 0.0139 & 0.0042 \\
\hline
\end{tabular}

Table 29: A2 efficiency dependence on A2 cut for L_KIN_3.

A2 EFFICIENCY FOR RUN 2509-2521 WITH RICH (Lambda)

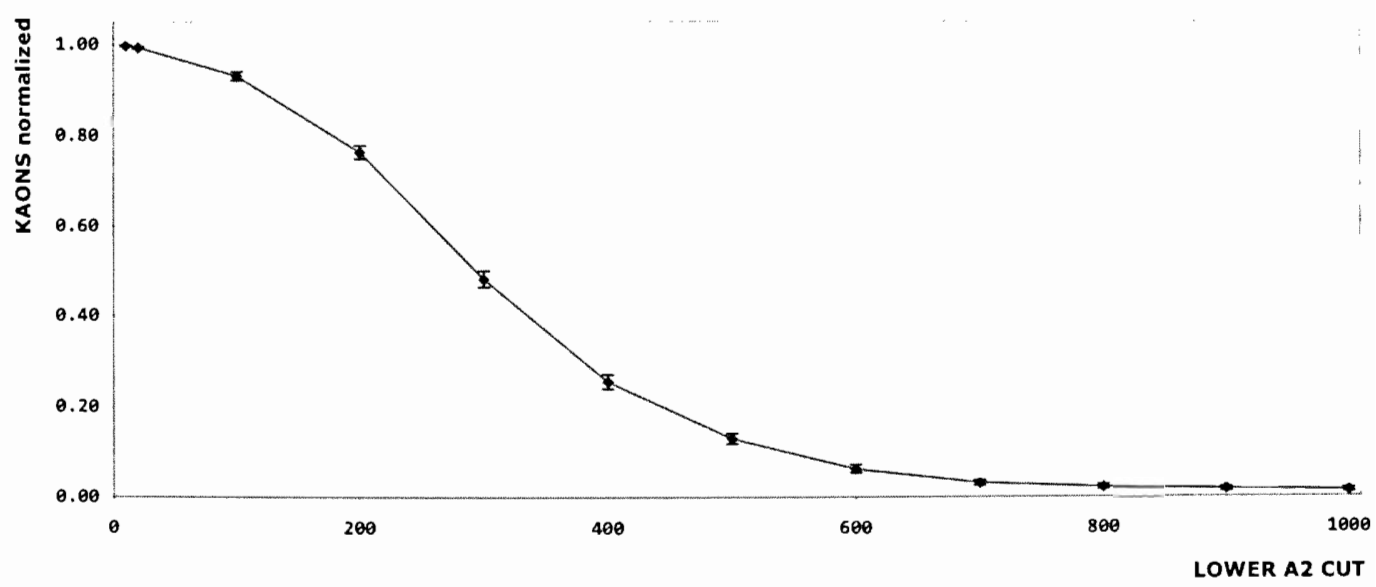

Figure 69: A2 efficiency dependence on A2 cut for L_KIN_3. 


\begin{tabular}{cccccc}
\hline & \multicolumn{5}{c}{$\begin{array}{c}\text { A2 }>100 \\
\text { and } \\
\text { A2<300 }\end{array}$} \\
\hline RICH+A2: & 70 & A1sum & 22 & 67 & \\
A1 CUT $(<)$ & RICH+AERO & & & \\
& ACCIDENTAL & & \\
& TOTAL & $\left({ }^{*} 10\right)$ & TRUE & normalized & uncertainty \\
-10 & 0 & 0 & 0 & 0 & 0.0000 \\
0 & 0 & 0 & 0 & 0 & 0.0000 \\
5 & 65 & 20 & 63 & 0.9403 & 0.0289 \\
10 & 67 & 20 & 65 & 0.9701 & 0.0208 \\
25 & 68 & 20 & 66 & 0.9851 & 0.0148 \\
50 & 68 & 21 & 65 & 0.9701 & 0.0208 \\
75 & 68 & 21 & 65 & 0.9701 & 0.0208 \\
100 & 68 & 21 & 65 & 0.9701 & 0.0208 \\
125 & 69 & 21 & 66 & 0.9851 & 0.0148 \\
150 & 69 & 21 & 66 & 0.9851 & 0.0148 \\
175 & 70 & 21 & 67 & 1.0000 & 0.0000 \\
200 & 70 & 21 & 67 & 1.0000 & 0.0000 \\
\hline
\end{tabular}

Table 30: A1 inefficiency dependence on A1 cut for S_KIN_1.

A1 inEFFICIENCY FOR RUN 2485-2492 WITH RICH (Sigma)

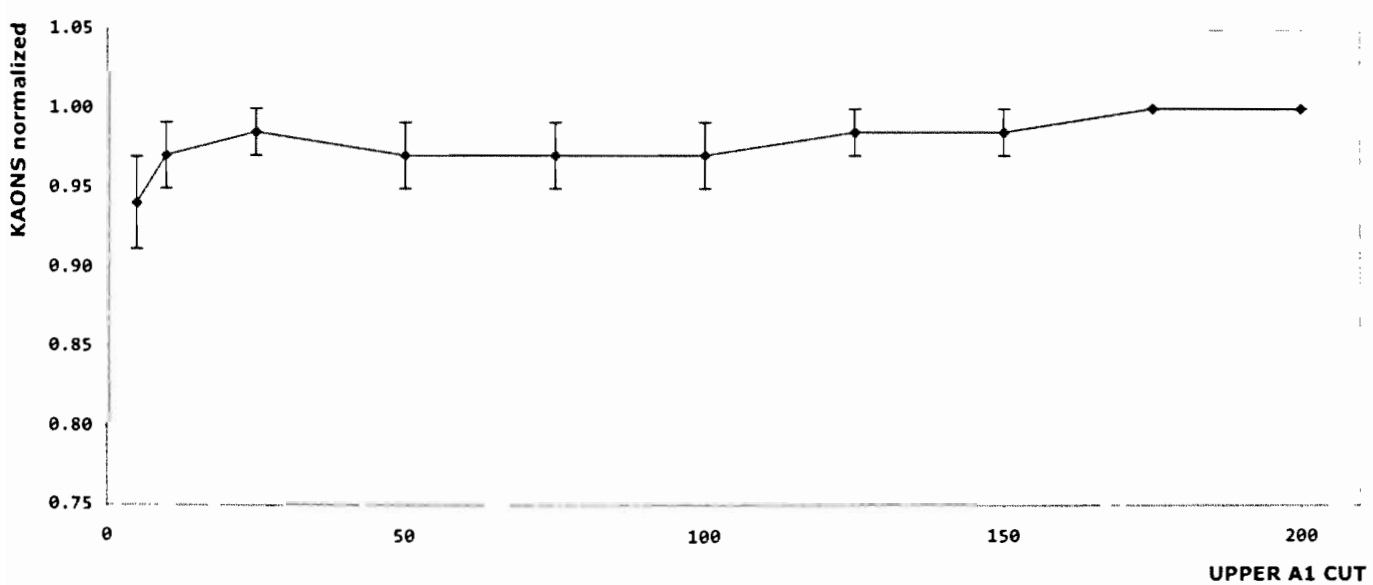

Figure 70: A1 inefficiency dependence on A1 cut for S_KIN_1. 


\begin{tabular}{|c|c|c|c|c|c|}
\hline KAONS(Sigma) & & Alsum & $\begin{array}{c}\mathrm{A} 2>100 \\
\text { and } \\
\mathrm{A} 2<300\end{array}$ & & \\
\hline $\mathrm{RICH}+\mathrm{A} 2$ : & 163 & 121 & 150 & & \\
\hline \multirow[t]{2}{*}{ Al CUT $(<)$} & $\mathrm{RICH}+\mathrm{AERO}$ & counts & & inefficiency & \\
\hline & TOTAL & $\begin{array}{l}\text { ACCIDENTAL } \\
(* 10)\end{array}$ & TRUE & normalized & uncertainty \\
\hline-10 & 0 & 0 & 0 & 0 & 0.0000 \\
\hline 0 & 0 & 0 & 0 & 0 & 0.0000 \\
\hline 5 & 140 & 104 & 129 & 0.8600 & 0.0283 \\
\hline 10 & 142 & 104 & 131 & 0.8733 & 0.0272 \\
\hline 25 & 144 & 105 & 133 & 0.8867 & 0.0259 \\
\hline 50 & 146 & 105 & 135 & 0.9000 & 0.0245 \\
\hline 75 & 149 & 106 & 138 & 0.9200 & 0.0222 \\
\hline 100 & 153 & 109 & 142 & 0.9467 & 0.0183 \\
\hline 125 & 157 & 110 & 146 & 0.9733 & 0.0132 \\
\hline 150 & 160 & 111 & 148 & 0.9867 & 0.0094 \\
\hline 175 & 160 & 111 & 148 & 0.9867 & 0.0094 \\
\hline 200 & 161 & 111 & 149 & 0.9933 & 0.0066 \\
\hline
\end{tabular}

Table 31: A1 inefficiency dependence on A1 cut for S_KIN_2.

A1 inEFFICIENCY FOR RUN 2496-2508 WITH RICH (Sigma)

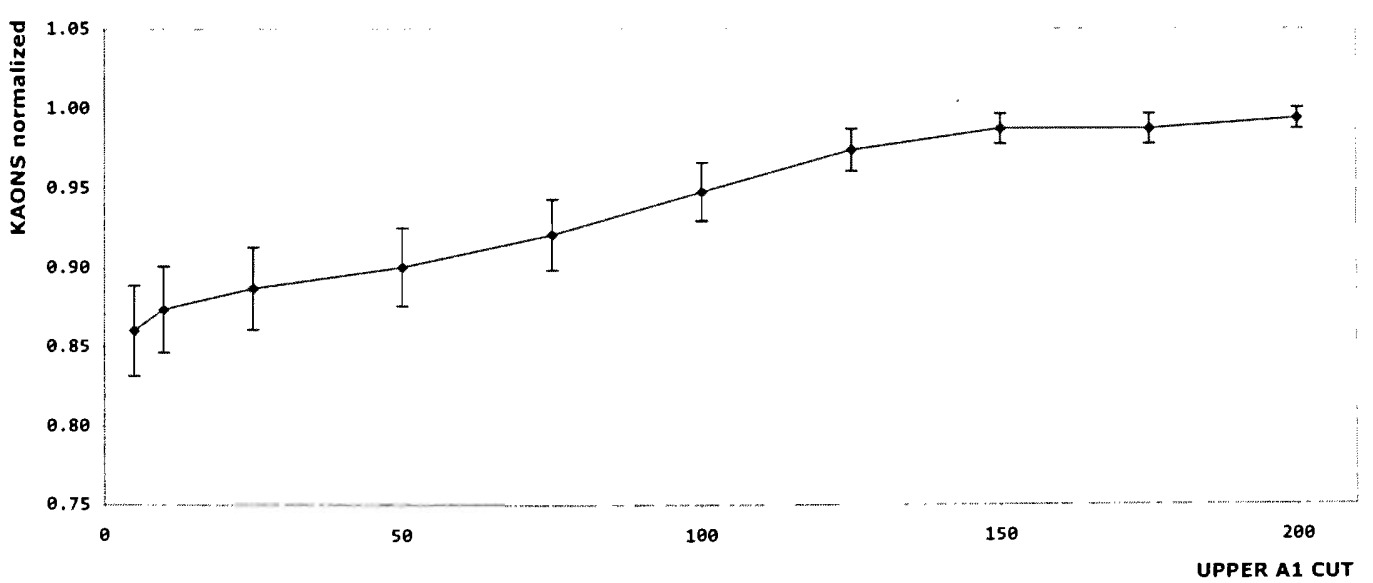

Figure 71: A1 inefficiency dependence on A1 cut for S_KIN_2. 


\begin{tabular}{|c|c|c|c|c|c|}
\hline KAONS(Sigma) & & A2sum & $\mathrm{A} 1<10$ & & \\
\hline $\mathrm{RICH}+\mathrm{A} 1:$ & 115 & 53 & 109 & & \\
\hline \multirow[t]{2}{*}{ A2 CUT $(>)$} & $\mathrm{RICH}+\mathrm{AERO}$ & counts & & efficiency & \\
\hline & TOTAL & $\begin{array}{c}\text { ACCIDENTAL } \\
(* 10)\end{array}$ & TRUE & normalized & uncertainty \\
\hline-10 & 115 & 53 & 109 & 1.0000 & 0.0000 \\
\hline 0 & 114 & 41 & 109 & 1.0000 & 0.0000 \\
\hline 10 & 114 & 40 & 110 & 1.0092 & 0.0092 \\
\hline 20 & 114 & 40 & 110 & 1.0092 & 0.0092 \\
\hline 100 & 98 & 38 & 94 & 0.8624 & 0.0330 \\
\hline 200 & 57 & 24 & 54 & 0.4954 & 0.0479 \\
\hline 300 & 31 & 18 & 29 & 0.2661 & 0.0423 \\
\hline 400 & 15 & 9 & 14 & 0.1284 & 0.0320 \\
\hline 500 & 7 & 8 & 6 & 0.0550 & 0.0218 \\
\hline 600 & 5 & 6 & 4 & 0.0367 & 0.0180 \\
\hline 700 & 5 & 4 & 4 & 0.0367 & 0.0180 \\
\hline 800 & 5 & 4 & 4 & 0.0367 & 0.0180 \\
\hline 900 & 3 & 3 & 2 & 0.0183 & 0.0129 \\
\hline 1000 & 3 & 3 & 2 & 0.0183 & 0.0129 \\
\hline
\end{tabular}

Table 32: A2 efficiency dependence on A2 cut for S_KIN_1.

A2 EFFICIENCY FOR RUN 2485-2492 WITH RICH (Sigma)

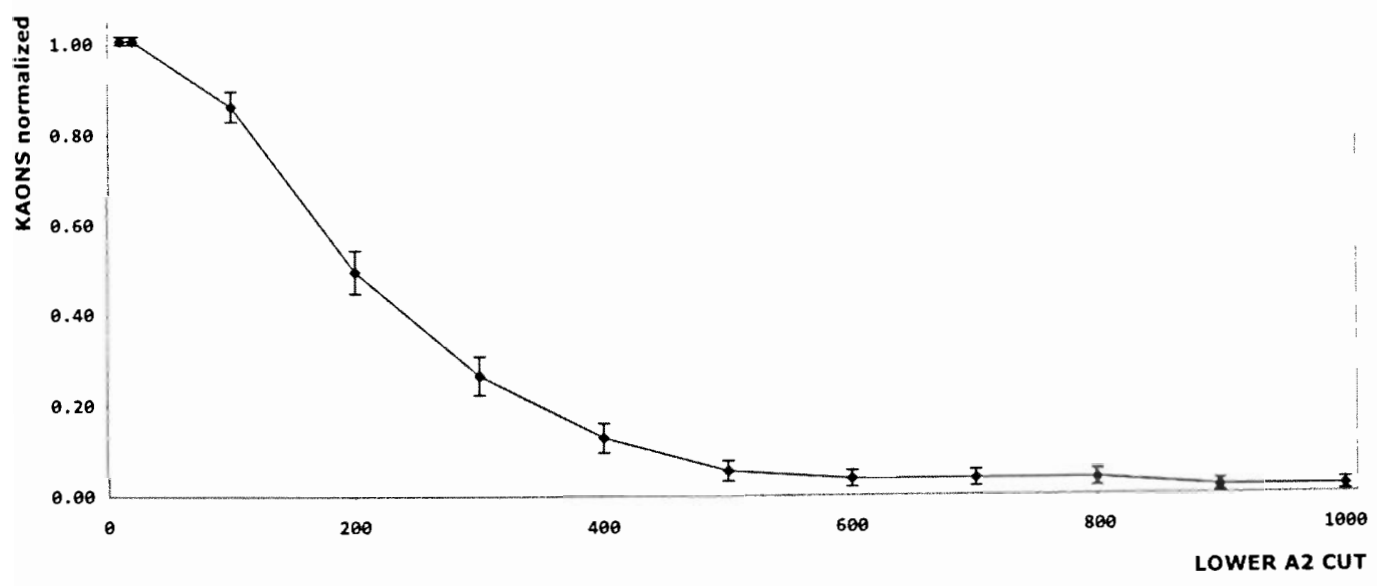

Figure 72: A2 efficiency dependence on A2 cut for S_KIN_1. 


\begin{tabular}{|c|c|c|c|c|c|}
\hline KAONS(Sigma) & & A2sum & $\mathrm{Al}<10$ & & \\
\hline $\mathrm{RICH}+\mathrm{A} 1:$ & 275 & 241 & 250 & & \\
\hline \multirow[t]{2}{*}{ A2 $\operatorname{CUT}(>)$} & $\mathrm{RICH}+\mathrm{AERO}$ & counts & & efficiency & \\
\hline & TOTAL & $\begin{array}{l}\text { ACCIDENTAL } \\
\left({ }^{*} 10\right)\end{array}$ & TRUE & normalized & uncertainty \\
\hline-10 & 275 & 241 & 250 & 1.0000 & 0.0000 \\
\hline 0 & 272 & 206 & 251 & 1.0040 & 0.0040 \\
\hline 10 & 272 & 201 & 251 & 1.0040 & 0.0040 \\
\hline 20 & 272 & 199 & 252 & 1.0080 & 0.0057 \\
\hline 100 & 244 & 183 & 225 & 0.9000 & 0.0190 \\
\hline 200 & 166 & 129 & 153 & 0.6120 & 0.0308 \\
\hline 300 & 102 & 79 & 94 & 0.3760 & 0.0306 \\
\hline 400 & 61 & 43 & 56 & 0.2240 & 0.0264 \\
\hline 500 & 27 & 29 & 24 & 0.0960 & 0.0186 \\
\hline 600 & 17 & 24 & 14 & 0.0560 & 0.0145 \\
\hline 700 & 10 & 15 & 8 & 0.0320 & 0.0111 \\
\hline 800 & 8 & 11 & 6 & 0.0240 & 0.0097 \\
\hline 900 & 6 & 7 & 5 & 0.0200 & 0.0089 \\
\hline 1000 & 3 & 4 & 2 & 0.0080 & 0.0056 \\
\hline
\end{tabular}

Table 33: A2 efficiency dependence on A2 cut for S_KIN_2.

A2 EFFICIENCY FOR RUN 2496-2508 WITH RICH (Sigma)

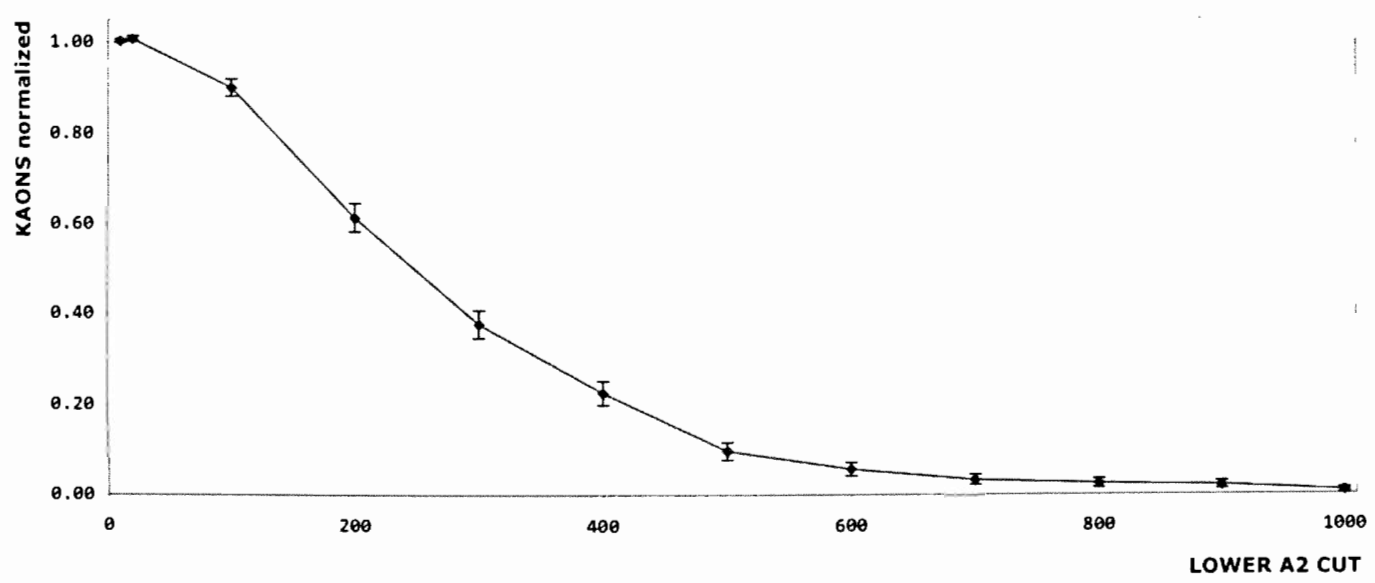

Figure 73: A2 efficiency dependence on A2 cut for S_KIN_2. 
In a similar way a RICH efficiency analysis was performed selecting tight cuts in the aerogels to ensure a clean sample of kaons according to Table 22 for the three different kinematics (see Table 19). The efficiencies are quite less than $100 \%$. The RICH low efficiencies cause a significant correction factor to the data as well as decreasing the statistics and increasing the statistical uncertainty. Therefore the RICH was instead taken as an extra detector to be used for the aerogel analysis but not for the cross section analysis. See Appendix G.

\subsection{Gas threshold Cherenkov detector analysis}

The $\mathrm{CO}_{2}$ gas Cherenkov is located in the right arm (see Section 2.6.3) and the purpose of it is to discriminate electrons from negative pions $\left(\pi^{-}\right)$. To perform the analysis of the efficiency of this detector, positive pions $\left(\pi^{+}\right)$in the left arm in coincidence with electrons in the right arm were used, which means the coincidence time for pions was selected (Table 19) for the three different kinematics (Table 15). The analysis also includes background subtraction (Section 4.2), RICH PID cut for pion selection (Table 17) and special tight cuts in the aerogels to select a clean sample of positive pions $\left(\pi^{+}\right)$in the left arm given in Table 34 .

\begin{tabular}{cc}
\hline Detector & Pion tight cut (channels) \\
\hline A1 & $>100$ \\
A2 & $>500$ \\
\hline
\end{tabular}

Table 34: Tight cuts applied to A1 and A2 to select clean sample of pions [68]. 
In addition to the previously mentioned cuts, it is also important to be sure that we have a clean sample of electrons in the right arm. To have a clean sample of electrons we made used of two extra detectors in the right arm; the shower and the preshower. If we plot shower ADC signal vs. the preshower ADC signal a so-called "banana" plot (because of its obvious resemblance) is obtained, where two "areas" with counts are seen. Electrons release more energy in the preshower and shower counters (counts at higher ADC channels) because of their lighter masses than negative pions, then it is possible to differentiate negative pions $\left(\pi^{-}\right)$from electrons $\left(e^{-}\right)$. Plotted is the energy deposited in the first layer or preshower versus the energy deposited in the rest of the detector [68]. The "banana" plots for the three different kinematics with the cuts applied to select electrons (preshower $\mathrm{ADC}>500$ and shower $\mathrm{ADC}>500$ ) in the right arm are shown in Figures 74,75 and 76.

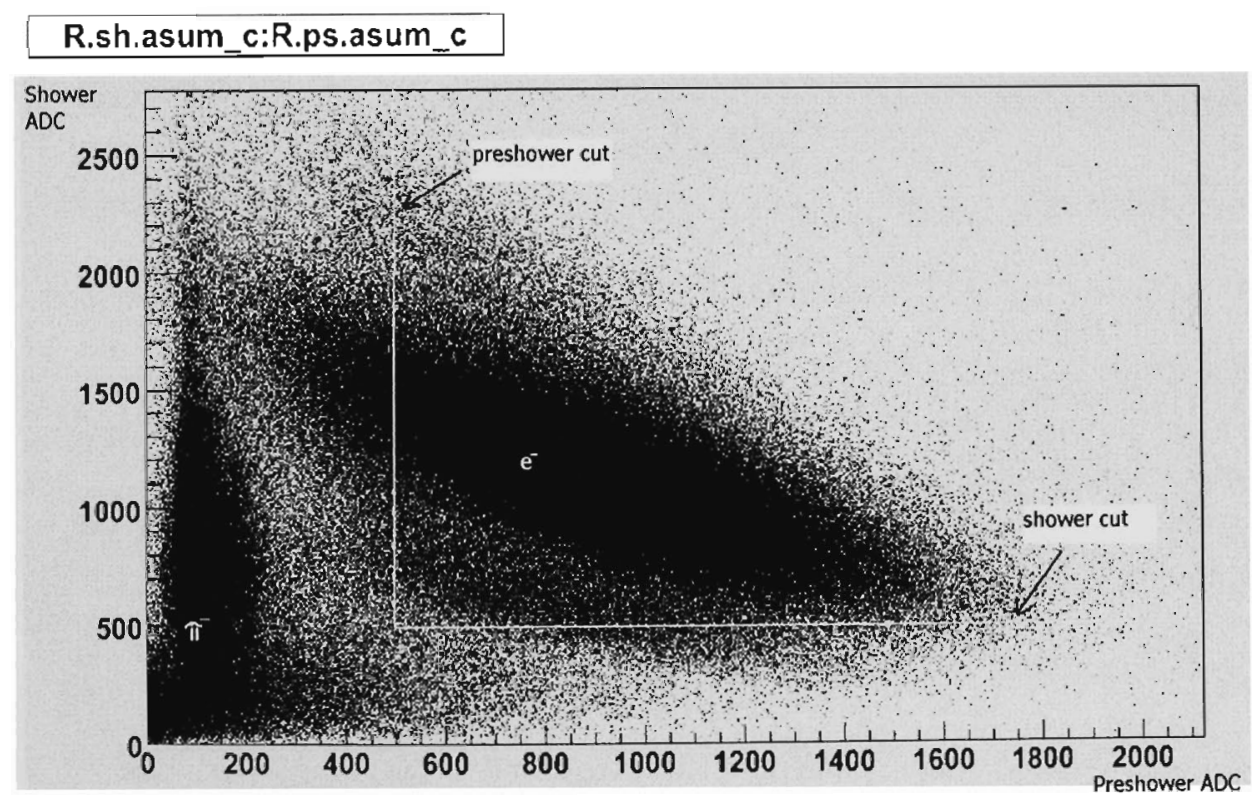

Figure 74: "Banana" plot for KIN_1, showing shower and preshower detector cuts. 


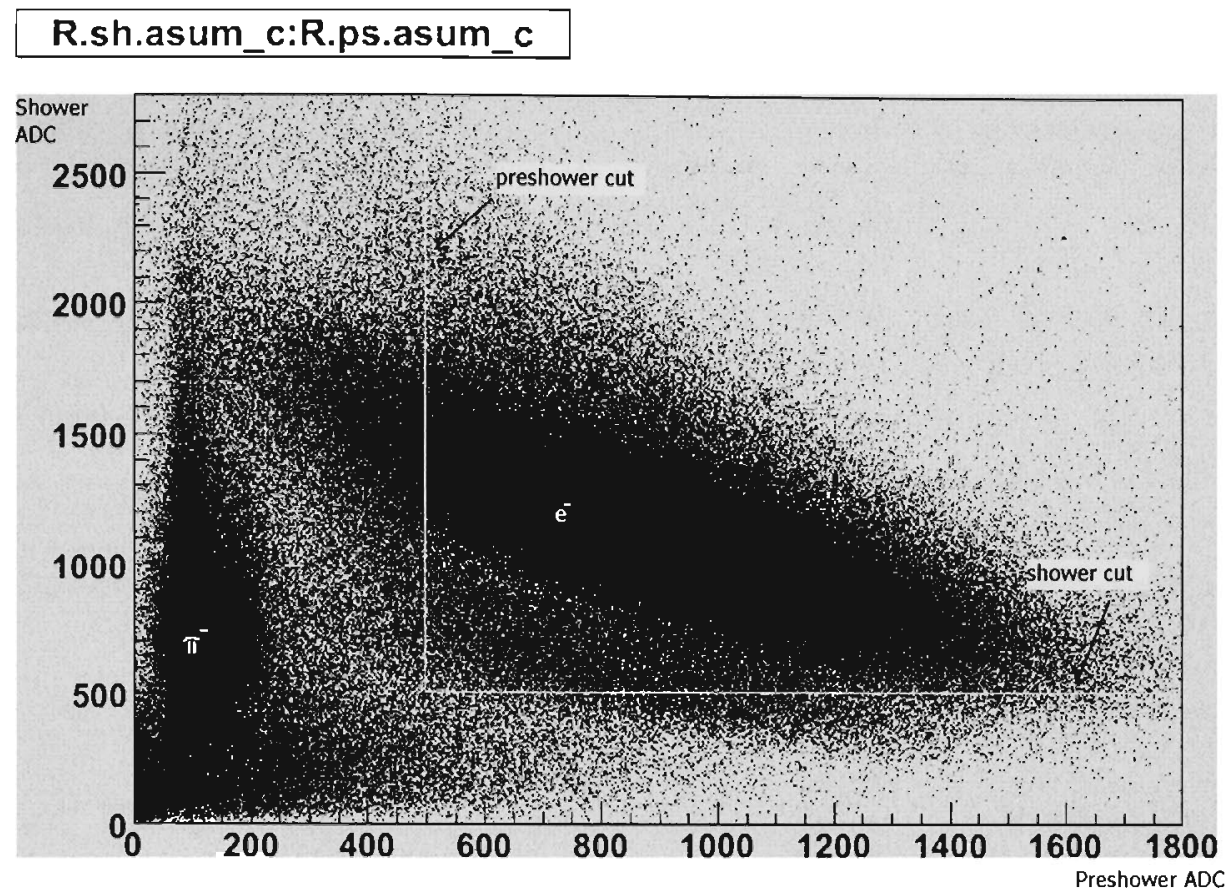

Figure 75: "Banana" plot for KIN_2, showing shower and preshower detector cuts.

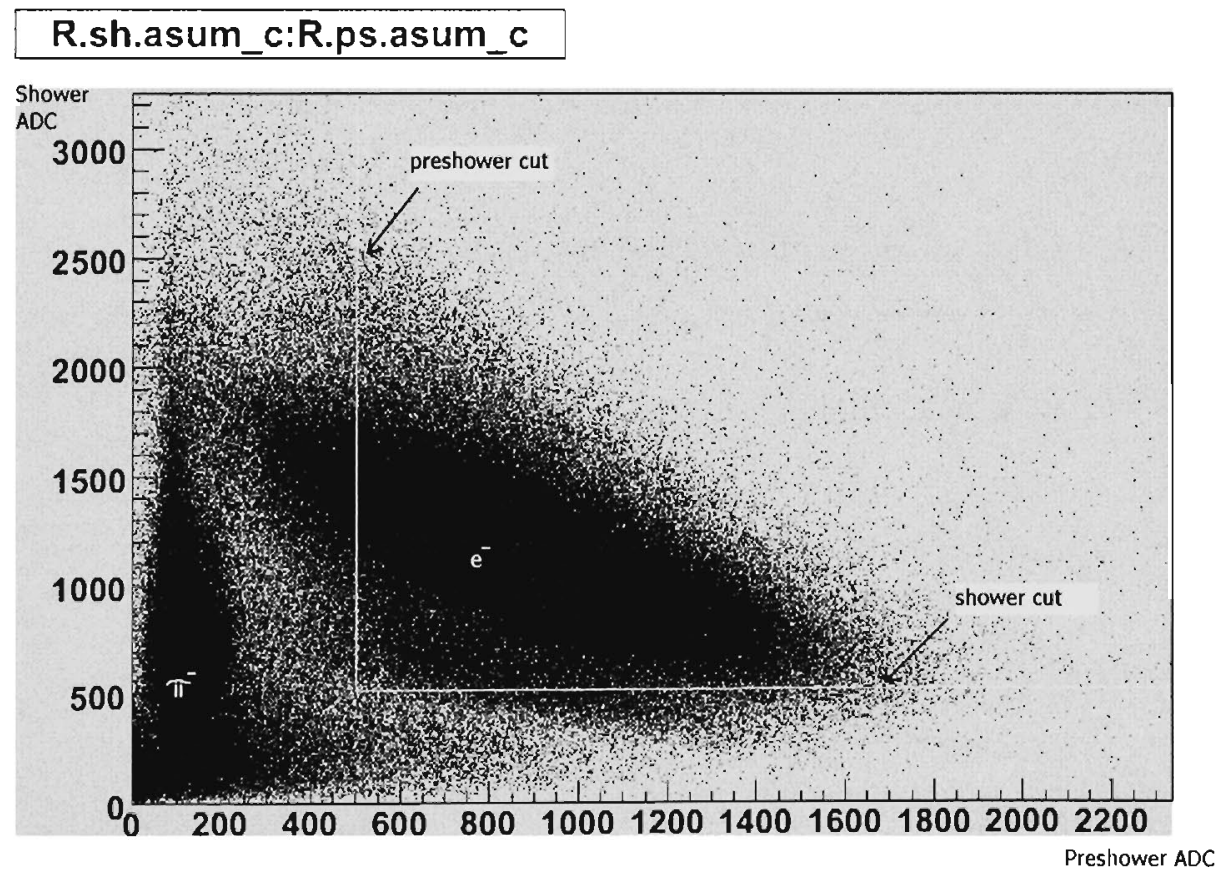

Figure 76: "Banana" plot for KIN_3, showing shower and preshower detector cuts. 
The use of pions instead of kaons has the advantage that the statistics are much larger, which gives a smaller statistical uncertainty on the measurement of the gas Cherenkov detector efficiency. The details of efficiency analysis for the different kinematics are shown in Tables 35-37 and Figures 77-79. More details of uncertainties calculations are shown in Section 4.9.

\begin{tabular}{|c|c|c|c|c|c|}
\hline coin PIONS & & RCERsum & $\mathrm{A} 1>100, \mathrm{~A} 2>500$ & & \\
\hline $\mathrm{RICH}+\mathrm{A} 1+\mathrm{A} 2:$ & 48175 & 118212 & 36353 & & \\
\hline $\mathrm{A} 2 \operatorname{CUT}(>)$ & $\mathrm{RICH}+\mathrm{AERO}$ & counts & & efficiency & \\
\hline & TOTAL & $\begin{array}{l}\text { ACCIDENTAL } \\
\left({ }^{*} 10\right)\end{array}$ & TRUE & normalized & uncertainty \\
\hline-10 & 48175 & 118212 & 36353 & 1.0000 & 0.0000 \\
\hline 0 & 47547 & 117456 & 35801 & 0.9848 & 0.0006 \\
\hline 10 & 47539 & 117445 & 35794 & 0.9846 & 0.0006 \\
\hline 200 & 47386 & 117093 & 35676 & 0.9814 & 0.0007 \\
\hline 400 & 46172 & 113832 & 34788 & 0.9569 & 0.0011 \\
\hline 600 & 41691 & 102408 & 31450 & 0.8651 & 0.0018 \\
\hline 800 & 32793 & 80673 & 24725 & 0.6801 & 0.0024 \\
\hline 1000 & 21820 & 54203 & 16399 & 0.4511 & 0.0026 \\
\hline 1200 & 12700 & 31424 & 9557 & 0.2629 & 0.0023 \\
\hline 1400 & 6596 & 16371 & 4958 & 0.1364 & 0.0018 \\
\hline 1600 & 3251 & 8019 & 2449 & 0.0674 & 0.0013 \\
\hline 1800 & 1549 & 4015 & 1147 & 0.0316 & 0.0009 \\
\hline 2000 & 802 & 2048 & 597 & 0.0164 & 0.0007 \\
\hline
\end{tabular}

Table 35: $\mathrm{CO}_{2}$ gas Cherenkov detector efficiency at different cuts in KIN_1.

CO2 CER EFFICIENCY FOR RUN 2485-2492 WITH coin PIONS

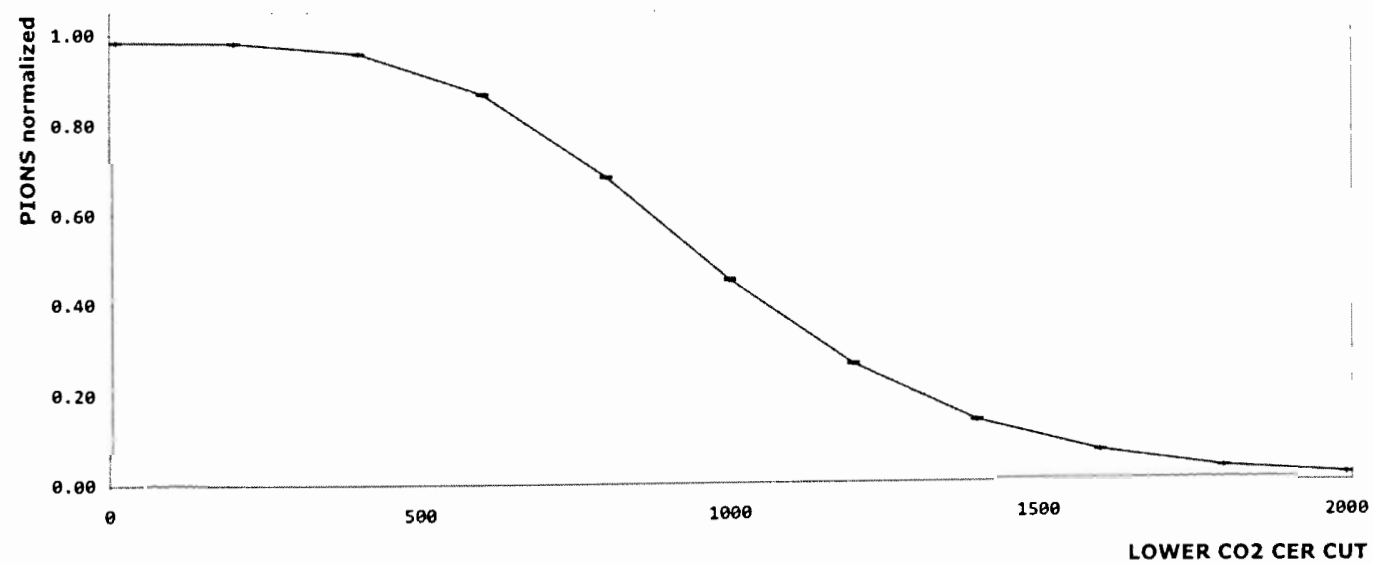

Figure 77: $\mathrm{CO}_{2}$ gas Cherenkov detector efficiency at different cuts in KIN_1. 


\begin{tabular}{|c|c|c|c|c|c|}
\hline coin PIONS & & RCERsum & $\mathrm{A} 1>100, \mathrm{~A} 2>500$ & & \\
\hline $\mathrm{RICH}+\mathrm{A} 1+\mathrm{A} 2:$ & 48895 & 137776 & 35117 & & \\
\hline A2 CUT (>) & $\mathrm{RICH}+\mathrm{AERO}$ & counts & \multicolumn{3}{|c|}{ efficiency } \\
\hline & & ACCIDENTAL & TDUE & & \\
\hline-10 & $\begin{array}{c}\text { TOTAL } \\
48895\end{array}$ & $\begin{array}{c}(* 10) \\
137776\end{array}$ & $\begin{array}{l}\text { TRUE } \\
35117\end{array}$ & $\begin{array}{c}\text { normalized } \\
1.0000\end{array}$ & $\begin{array}{c}\text { uncertainty } \\
0.0000\end{array}$ \\
\hline 0 & 48264 & 136934 & 34570 & 0.9844 & 0.0007 \\
\hline 10 & 48260 & 136928 & 34567 & 0.9843 & 0.0007 \\
\hline 200 & 48115 & 136507 & 34464 & 0.9814 & 0.0007 \\
\hline 400 & 46809 & 132647 & 33544 & 0.9552 & 0.0011 \\
\hline 600 & 41924 & 118820 & 30042 & 0.8555 & 0.0019 \\
\hline 800 & 32917 & 92906 & 23626 & 0.6728 & 0.0025 \\
\hline 1000 & 22054 & 62221 & 15831 & 0.4508 & 0.0027 \\
\hline 1200 & 12727 & 35832 & 9143 & 0.2604 & 0.0023 \\
\hline 1400 & 6590 & 18550 & 4735 & 0.1348 & 0.0018 \\
\hline 1600 & 3261 & 9100 & 2351 & 0.0669 & 0.0013 \\
\hline 1800 & 1670 & 4471 & 1222 & 0.0348 & 0.0010 \\
\hline 2000 & 886 & 2237 & 662 & 0.0189 & 0.0007 \\
\hline
\end{tabular}

Table 36: $\mathrm{CO}_{2}$ gas Cherenkov detector efficiency at different cuts in KIN_2.

CO2 CER EFFICIENCY FOR RUN 2496-2508 WITH coin PIONS

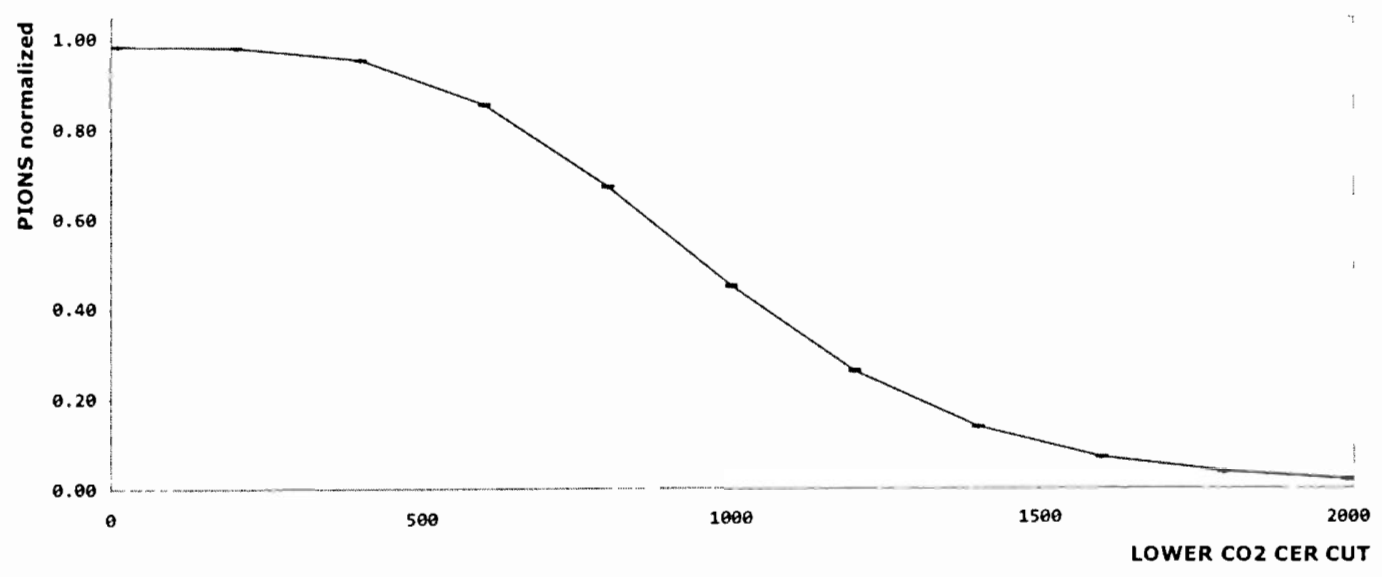

Figure 78: $\mathrm{CO}_{2}$ gas Cherenkov detector efficiency at different cuts in KIN_2. 


\begin{tabular}{|c|c|c|c|c|c|}
\hline coin PIONS & & RCERsum & $\mathrm{A} 1>100, \mathrm{~A} 2>500$ & & \\
\hline $\mathrm{RICH}+\mathrm{A} 1+\mathrm{A} 2:$ & 30775 & 117191 & 19055 & & \\
\hline $\mathrm{A} 2 \mathrm{CUT}(>)$ & $\mathrm{RICH}+\mathrm{AERO}$ & counts & & efficiency & \\
\hline & TOTAL & $\begin{array}{l}\text { ACCIDENTAL } \\
(* 10)\end{array}$ & TRUE & normalized & uncertainty \\
\hline-10 & 30775 & 117191 & 19055 & 1.0000 & 0.0000 \\
\hline 0 & 30263 & 116459 & 18617 & 0.9770 & 0.0011 \\
\hline 10 & 30260 & 116457 & 18614 & 0.9769 & 0.0011 \\
\hline 200 & 30159 & 116089 & 18550 & 0.9735 & 0.0012 \\
\hline 400 & 29341 & 112762 & 18064 & 0.9480 & 0.0016 \\
\hline 600 & 26439 & 101015 & 16337 & 0.8574 & 0.0025 \\
\hline 800 & 20659 & 78573 & 12801 & 0.6718 & 0.0034 \\
\hline 1000 & 13724 & 52079 & 8516 & 0.4469 & 0.0036 \\
\hline 1200 & 7788 & 29880 & 4800 & 0.2519 & 0.0031 \\
\hline 1400 & 3976 & 15196 & 2456 & 0.1289 & 0.0024 \\
\hline 1600 & 1893 & 7416 & 1151 & 0.0604 & 0.0017 \\
\hline 1800 & 925 & 3690 & 556 & 0.0292 & 0.0012 \\
\hline 2000 & 461 & 1865 & 274 & 0.0144 & 0.0009 \\
\hline
\end{tabular}

Table 37: $\mathrm{CO}_{2}$ gas Cherenkov detector efficiency at different cuts in KIN_3.

CO2 CER EFFICIENCY FOR RUN 2509_2521 WITH coin PIONS

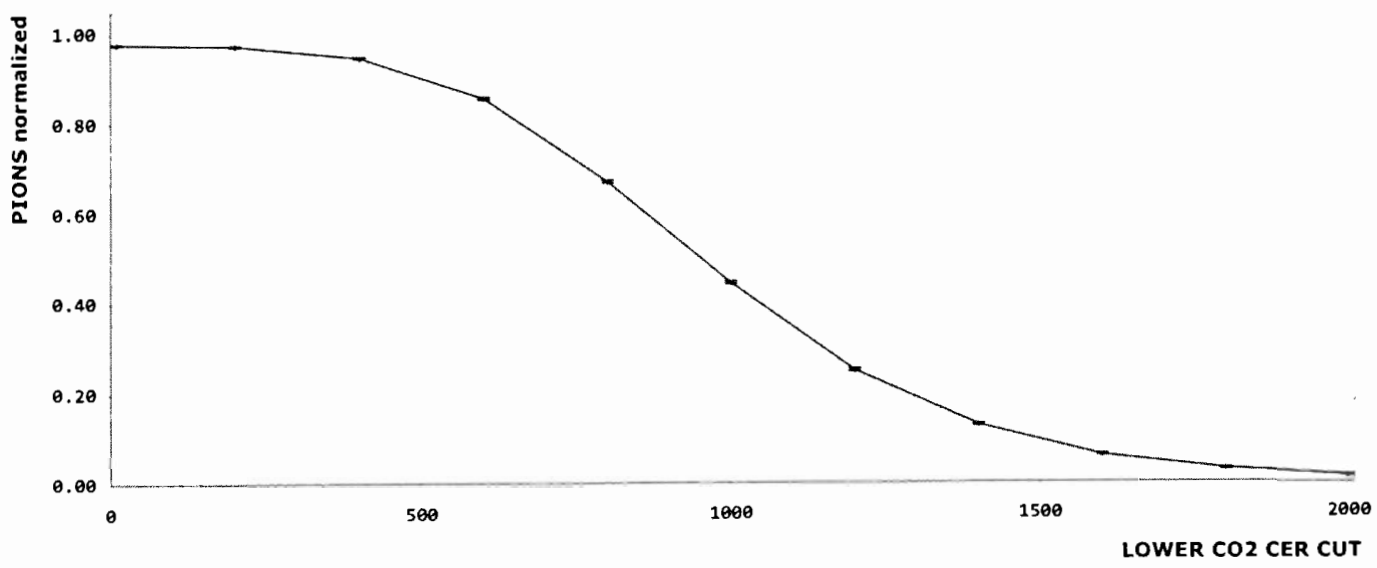

Figure 79: $\mathrm{CO}_{2}$ gas Cherenkov detector efficiency at different cuts in KIN_3. 
Another important ingredient to extract the cross section from the data for hyperon production in the elementary reaction is to simulate the experiment using Monte Carlo method. The standard Hall A Monte Carlo simulation (MCEEP) was convenient for this purpose.

\subsubsection{MCEEP description}

The MCEEP code was originally developed to simulate and calculate the cross section of the exclusive coincidence electron-nucleon reaction $\left(e, e^{\prime} N\right)$ [74]. However, later it was enhanced to also simulate and calculate the coincidence $\left(e, e^{\prime} K^{+}\right)[73]$ reaction cross section [72]. The MCEEP simulation includes: averaging over a theoretical model over the experimental acceptance; radiative and kaon decay corrections, Fermi motion, physics weighting, a realistic description of the beam, target, and detectors as well as an absolute normalization for the simulated events [72].

Among the capabilities of the MCEEP simulation is the effect of the raster of the beam and the three-dimensional interaction of the target with the beam; therefore the length of the targets is also required input in the simulation.

The MCEEP simulation also takes into account radiative corrections and realistic multiple scattering. Both internal and external radiative corrections (particle radiation before and after the vertex of the reactions) are performed. 
An event constitutes any combination of variables that satisfies the conditions for the reaction studied in the laboratory system of reference. The events are generated randomly within the spectrometer experimental acceptance. Each event is characterized by assigning a random momentum and two orientation angles to an emerging particle. The characterization of particles is performed for particles in both spectrometers (i.e., electrons and hadrons). Therefore, a single event includes six coordinates; a momentum and two angle coordinates for a hadron (left arm) and similarly for an electron (right arm). These variables can be weighted not just by phase space but if the user wishes they can also be weighted by the physics model cross section embedded in the MCEEP simulation.

The MCEEP simulation was used to calculate the yield ( $\mathrm{Y}_{\text {MCEEP }}$ ) of kaon electroproduction for the reaction $\left(e, e^{\prime} K^{+}\right)$by determining the cross section event by event. The kaon electroproduction cross sections were extracted by comparison of the simulated yield with the measured yield for both hyperons $\Lambda$ and $\Sigma^{0}$. Therefore in this case, MCEEP was used to calculate the yield considering the scattering-to-discrete states of the residual system, which in this case are the hyperons $\Lambda$ and $\Sigma^{0}$. A cut was applied in the simulation to select each of the two hyperons. The missing mass cuts in the MCEEP used were the same as in the data analysis shown in Table 20. The yield in MCEEP is then obtained by integrating the cross section over a five-fold acceptance volume:

$$
Y_{M C E E P}=L \int_{\Delta V} \frac{d^{5} \sigma}{d E^{\prime} d \Omega d \Omega} d E^{\prime} d \Omega{ }^{+} d \Omega K^{+}
$$


In Equation (48) $L$ is the luminosity given by:

$$
L=\frac{\rho \ell_{T} N_{A} Q}{e A} .
$$

In Equation (49) $\rho$ and $\ell_{T}$ are the target density and length, $N_{A}$ is Avogadro's number, $Q$ is the total integrated beam charge, $e$ is the fundamental charge constant of the electron, and $A$ is the atomic weight.

The integration in (48) is performed over the electron $\left(\Omega_{e}\right)$, kaon $\left(\Omega_{K^{+}}\right)$solid angles, and over the electron final energy $E^{\prime}$.

\subsubsection{Radiative corrections}

There are some corrections that have to be considered for the one-photon exchange diagram for the $\left(e, e^{\prime} N\right)$ reaction. Charged particles interact with the Coulomb field of the nucleus by radiating real photons through the scattering process, which is called internal bremsstrahlung. When a similar process occurs with nuclei other than the one involved in the scattering process then it is called external bremsstrahlung, and the correction in this case is done by using the Bethe-Bloch formula for the energy loss by collisions [72]. The emission and reabsorption of virtual photons are taken into consideration in the simulation. It implies the emission of real low energy photons (soft photons), whose energies are small compared to the energy of the incoming and outgoing electrons. 
The radiated photons affect the cross section by affecting the energy and momentum transfer in the reaction. The correction makes the simulation more realistic giving a better comparison with the experiment. Another type of correction included is the Schwinger correction, which considers real and virtual photons for the contribution of the radiative tail. The latter correction is also enhanced with an extra multiphoton correction that accounts for mutiple photon emission and reabsorption [72].

\subsubsection{Physics models}

In the MCEEP simulation the phase-space variables are weighted by the physics model. The simulation allows the usage of different models by opting for the kind of reaction the user is interested in. In the case of $\left(e, e^{\prime} K^{+}\right)$reactions the option is the "700". For the "700" option the physics model embedded was initially based on experimental fitting to the existing data prior to 1980 [11]. In this experimental fitting the cross section is parameterized by a couple of scaling factors given by [11]:

$$
\frac{d^{2} \sigma}{d \Omega_{K^{+}}^{*}} \sim f\left(Q^{2}\right) \times f(W),
$$

where these scaling factors are given by the equations:

$$
f\left(Q^{2}\right)=\frac{1}{\left(Q^{2}+X\right)^{2}} \quad \text { and }
$$




$$
f(W)=\frac{\left|\bar{p}_{K} \cdot\right|}{W\left(W^{2}-m_{p}^{2}\right)},
$$

where $X=2.67(\mathrm{GeV} / \mathrm{c})^{2}$ for the $\Lambda$ channel and $X=0.79(\mathrm{GeV} / \mathrm{c})^{2}$ for the $\Sigma^{0}$ channel. However, in the later experiment carried out about ten years ago it was found the scaling with $W$ at low $W$ was incorrect as shown by the solid line in Figure 80 .

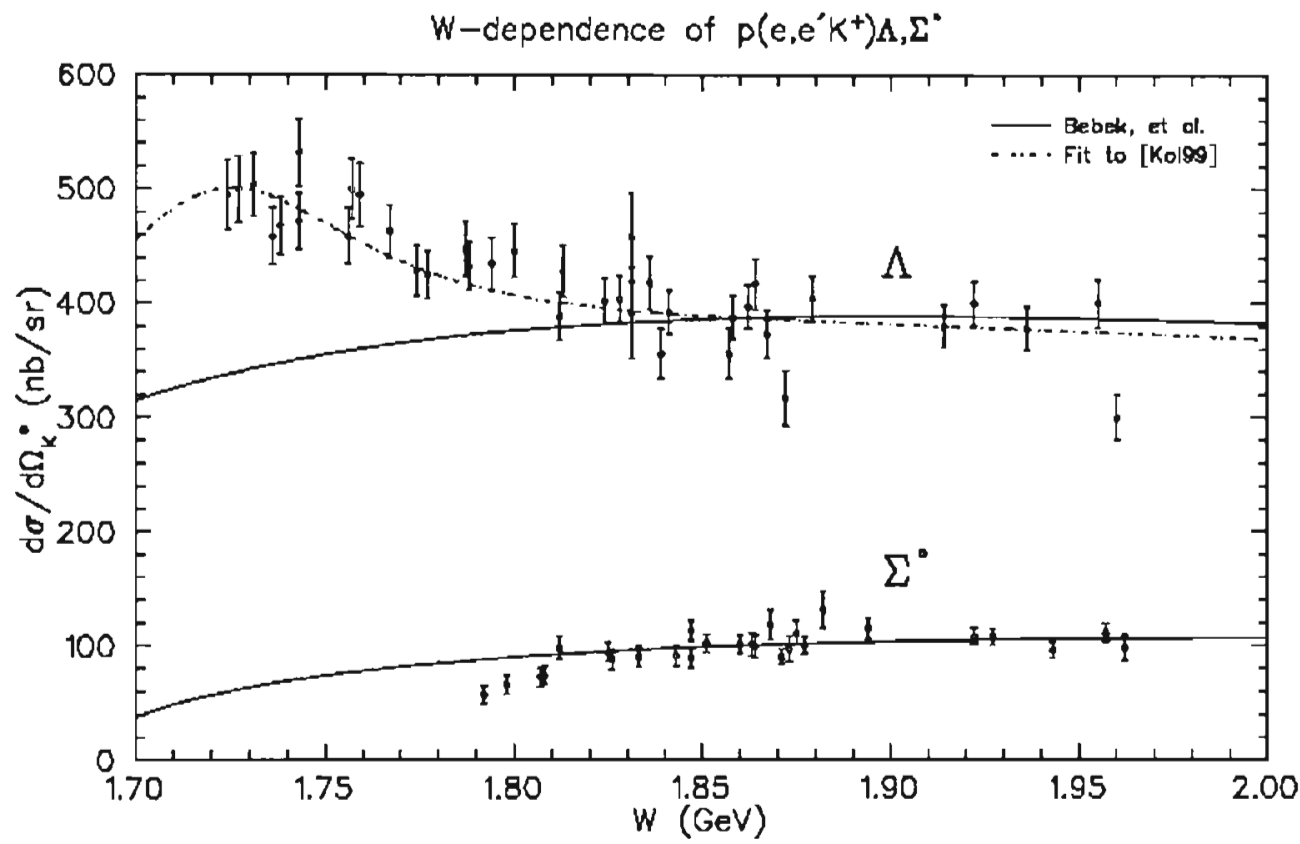

Figure 80: Cross sections as functions of $W$ with different scaling functions, $f(W)$, overlaid [75].

The embedded scaling functions in the MCEEP were provided by Bebek (solid line in Figure 80 ) while analysis results done in JLab experiment by D.M. Koltenuk for experiment E91-016 shows a better scaling function for the simulation [75]. The improved scaling function performed for the $\Lambda$ channel is given by: 


$$
f(W)=\frac{C_{1}\left|\bar{p}_{K^{+}}\right|}{W\left(W^{2}-m_{p}^{2}\right)}+\frac{C_{2}(1.72)^{2}(0.10)^{2}}{\left(W^{2}-(1.72)^{2}\right)^{2}+(1.72)^{2}(0.10)^{2}},
$$

here $C_{l}=4023.9 \mathrm{GeV}^{2} \mathrm{nb} / \mathrm{sr}$ and $C_{2}=180.0 \mathrm{GeV}^{2} \mathrm{nb} / \mathrm{sr}$. The corrected Equation (53) was estimated by considering possible resonance contributions to the cross section at lower $W$ that modify Equation (52).

Another scaling function was also taken into account later to correct the ones used initially in MCEEP. The scaling function is used to estimate the behavior of the cross section with $\theta_{C M}$. On the basis of the results by Brauel et al., [76], the scaling function can be shown to be [25]:

$$
\frac{f\left(\theta_{C M}\right)}{\left\langle f\left(\theta_{C M}=0^{0}\right)\right\rangle}=\frac{e^{-\xi|t|}}{e^{-\xi\left|t_{\min }\right|}}=e^{-\xi\left(t-t_{\min }\right)}
$$

with Mandelstam $t$,

$$
-t=-Q^{2}+m_{K^{+}}^{2}-2 E_{K^{+}}^{*} v^{*}+2\left|\bar{q}^{*}\right|\left|\bar{p}_{K^{+}}^{*}\right| \cos \left(\theta_{C M}\right),
$$

here $-t \rightarrow-t_{\min }$ when $\theta_{C M} \rightarrow 0^{0}$. The variables used in the equations of Section 4.6.3 were previously defined in Chapter 1.3. The starred variables refer to the $\mathrm{CM}$ frame.

MCEEP contains the latest corrections and it will be shown later in the next sections that it does a very good job in the comparison with the yields. One of the reasons 
is that, experiment $\mathrm{E} 94-107$ is performed at a higher $W \approx 2.2 \mathrm{GeV}$ where the model does a good job of describing the data compared to the data shown in Figure 80. However, the later corrections do not change the results by much in the kinematical region of experiment E94-107.

\subsection{Spectrometer acceptance corrections}

As was shown in Table 21 there are five cases to study that combine three different momenta in the hadron arm and two hyperon channels. The acceptance correction is performed for each of these cases with the purpose of excluding undesired events because of the edges of the entrance to the spectrometers where the acceptance changes rapidly. By comparing the MCEEP yield versus the measured yield it is possible to find the correction for the acceptance.

The MCEEP runs with many statistics, but the results must be scaled down so the amount of charge is the same as in the real experiment in order to get the same yields, which can be performed by giving the proper running time in MCEEP, and the proper luminosity so the integrated charges match. However, since the MCEEP simulation does not include detector efficiencies, for the purpose of comparing both yields the running time was deliberately changed to make the yields the same.

There were several details that must be taken into account when adapting the MCEEP input file to a particular experiment. Among them, the need to make sure that the coordinate $y_{\operatorname{tg}}$ matches the experimental value. Resolution spreading of the VDC resolution was adjusted so that the MCEEP $y_{t g}$ distribution matched $y_{t g}$ for real data. It 
was found that the missing mass peak was off by about $1 \sim 2 \mathrm{MeV}$. The reason for this was because of some small uncertainty in the accelerator (EPICS) data stream reading of the beam energy [38]. The database was corrected for that small amount of energy and the data were analyzed again. Because this experiment had essentially a point target (only 0.4 $\mathrm{cm}$ thick as seen by the spectrometers), the complexity of this correction procedure was reduced. By comparing both the measured yield versus the MCEEP yield with their integrals normalized it can be seen whether the two match. Then a small cut in the angular variables was applied watching carefully how all other variables vary. The idea is to apply the minimum cuts to have a good match and in this way avoid reducing the statistics by too much.

The comparison (histograms) of the two yields for the five different cases are shown in the next pages. The variables have been plotted such that in the first two rows the left or kaon spectrometer variables are to the left and the right or electron spectrometer variables are to the right while the last row show some of the reconstructed physics and coincidence variables. The angles in the first row are in radians and the $y_{t g}$ is in meters. The reconstructed variables are: missing energy in $\mathrm{GeV}$, the square of invariant mass $W$ in $\mathrm{GeV}^{2}$, the square of the four-momentum transfer $Q^{2}(\mathrm{GeV} / \mathrm{c})^{2}$ and $\theta_{C M}$ in radians. They are shown such that dash lines represent the MCEEP yield and solid lines represent the measured yield.

From Figure 81 to 85 the comparison between MCEEP yield and measured yield are shown with areas made equal (for comparison purposes), then from Figure 86 to 90 the comparison is shown after cuts made to the angles and/or momenta to make the acceptance correction. The kinematic/particle selections are given in Table 21 . 
The histograms shown in Figures 81-85 and 86-90 show a comparison between the MCEEP simulation (dashed line) and the real data (solid line). The first two rows are the simulated and reconstructed target variables for both spectrometers, where kaon refers to the left arm and electron refers to the right arm, so that;

$$
\begin{array}{ll}
\text { kaon phi }=\phi_{k}(\mathrm{rad}), & \text { kaon theta }=\theta_{k}(\mathrm{rad}), \\
\text { electron phi }=\phi_{e}(\mathrm{rad}), & \text { electron theta }=\theta_{e}(\mathrm{rad}), \\
\text { kaon } \mathrm{dp} / \mathrm{p}=(\delta p / p)_{K}, & \text { kaon } \mathrm{y}=\left(y_{t g}\right)_{K}, \\
\text { electron } \mathrm{dp} / \mathrm{p}=(\delta p / p)_{e}, & \text { electron } \mathrm{y}=\left(y_{t g}\right)_{e},
\end{array}
$$

the third row shows some of the reconstructed variables (see Section 1.3 for variable definitions);

$$
\begin{array}{ll}
\text { missing energy }=E_{\text {miss }}(\mathrm{GeV}), & \text { invariant mass } \mathrm{W}^{\wedge} 2=W^{2}\left(\mathrm{GeV}^{2}\right), \\
4 \text { momentum transfer } \mathrm{Q}^{\wedge} 2=Q^{2}\left(\mathrm{GeV}^{2}\right), & \text { theta in } \mathrm{CM}=\theta_{\mathrm{CM}}(\mathrm{rad}) .
\end{array}
$$

In Figures 91 to 95 extra reconstructed variables are shown;

$$
\begin{array}{ll}
\text { Mandelstam } \mathrm{t}=t\left(\mathrm{GeV}^{2}\right), & \text { phi_x }=\varphi(\mathrm{rad}), \\
\cos \left(\mathrm{phi} \_\mathrm{x}\right)=\cos (\varphi), & \sin \left(\mathrm{phi} \_\mathrm{x}\right)=\sin (\varphi), \\
\text { Electron } \mathrm{p}=p_{e}(\mathrm{GeV} / \mathrm{c}), & \text { Kaon } \mathrm{p}=p_{K}(\mathrm{GeV} / \mathrm{c}), \\
\text { Theta } \mathrm{Kq}=\theta_{K}(\mathrm{rad}), & \text { Theta_Lambdaq }=\theta_{Y}(\mathrm{rad}), \\
\text { Omega }=E-E^{\prime}(\mathrm{GeV}), & \text { Theta_lab } \mathrm{q}=\operatorname{angle}\left(\vec{p}_{e}, \vec{q}\right)(\mathrm{rad}), \\
\text { Electron scattering angle }=\theta_{e}(\mathrm{rad}), & \text { Epsilon }=\varepsilon .
\end{array}
$$


\begin{tabular}{|l|l|}
\hline kaon phi & Entries 188667 \\
\hline & Mean -0.0006204 \\
\hline
\end{tabular}

$\begin{array}{ll}\infty & \end{array}$
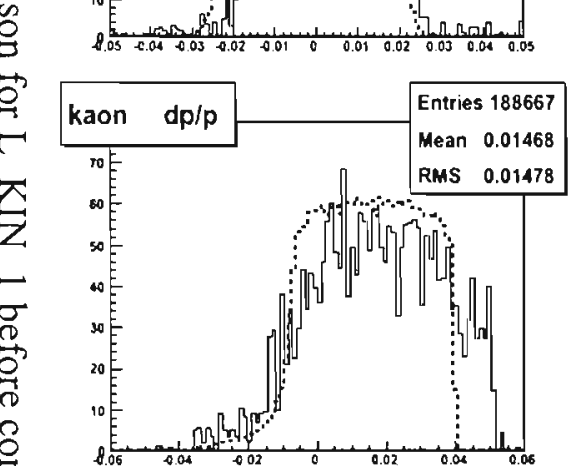

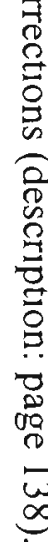

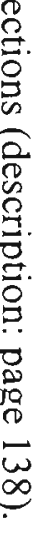
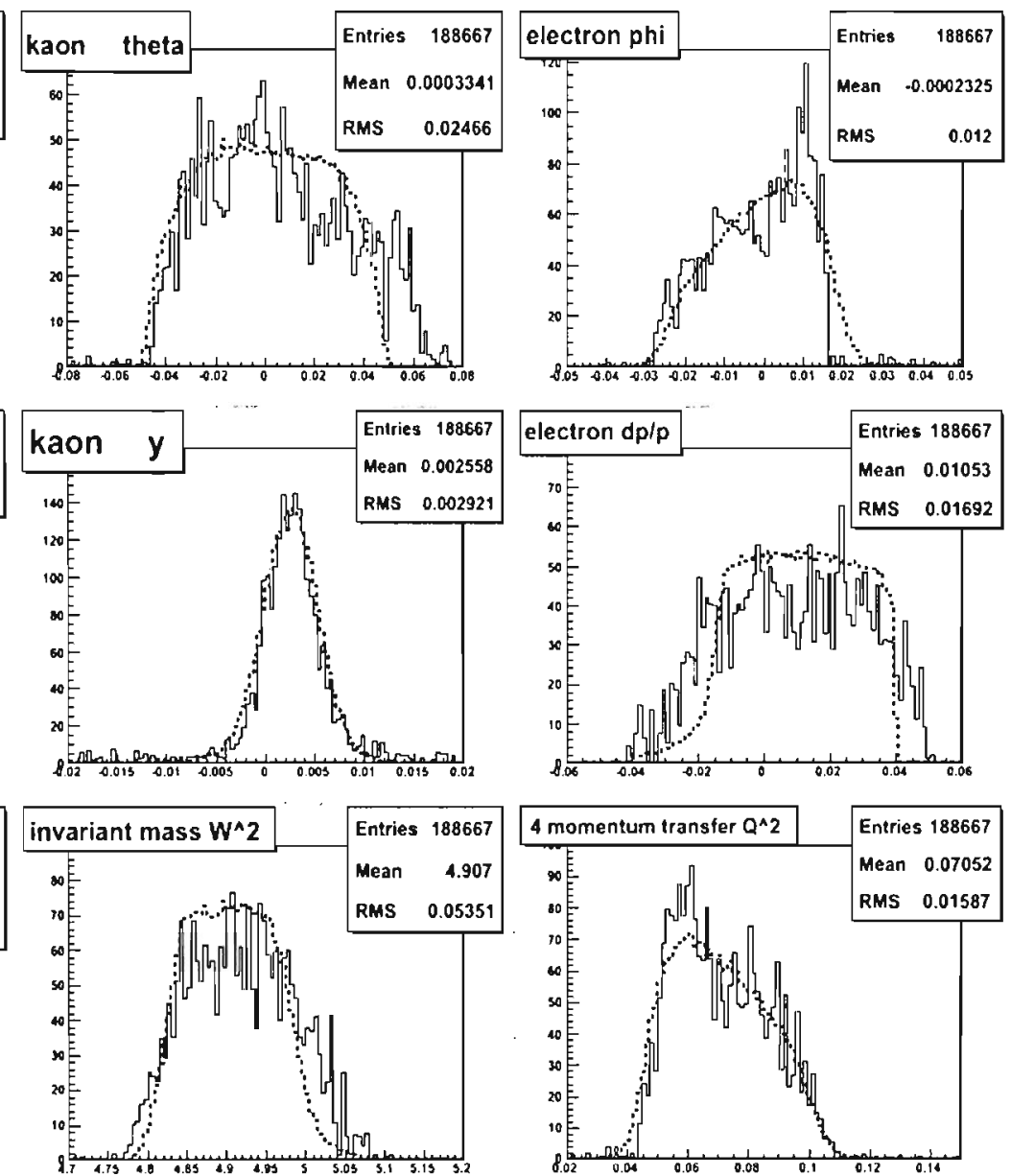
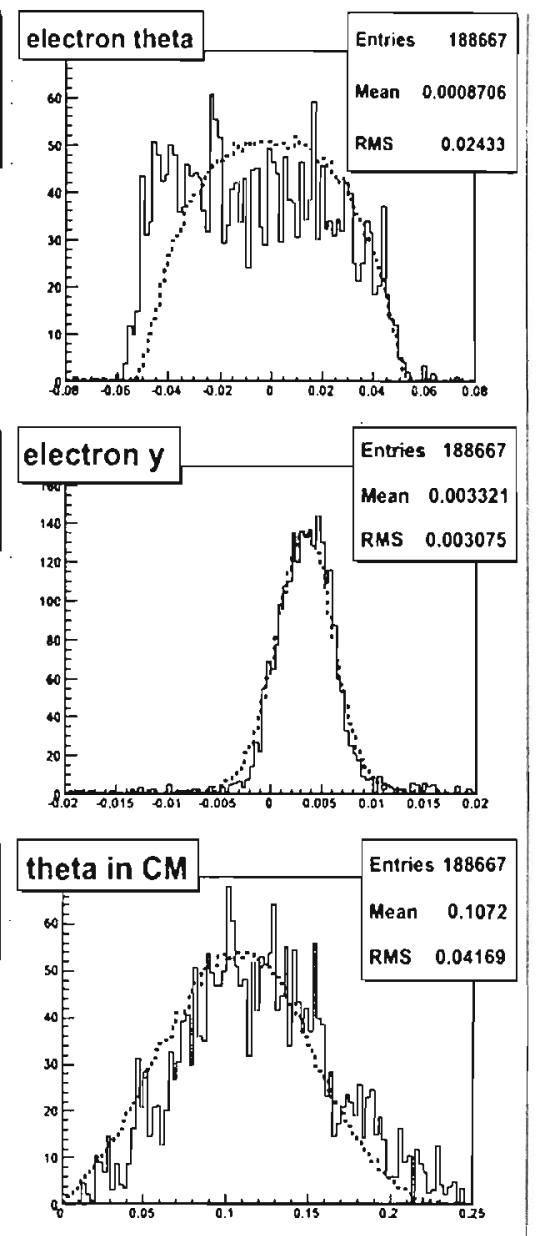

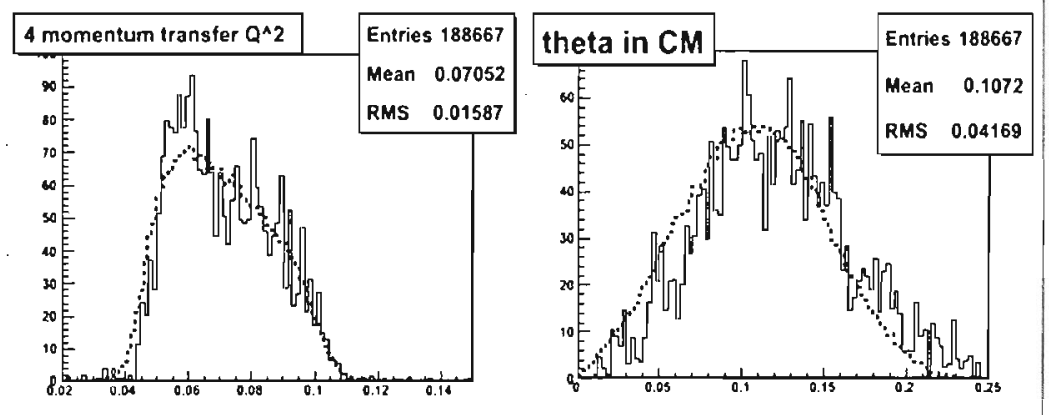




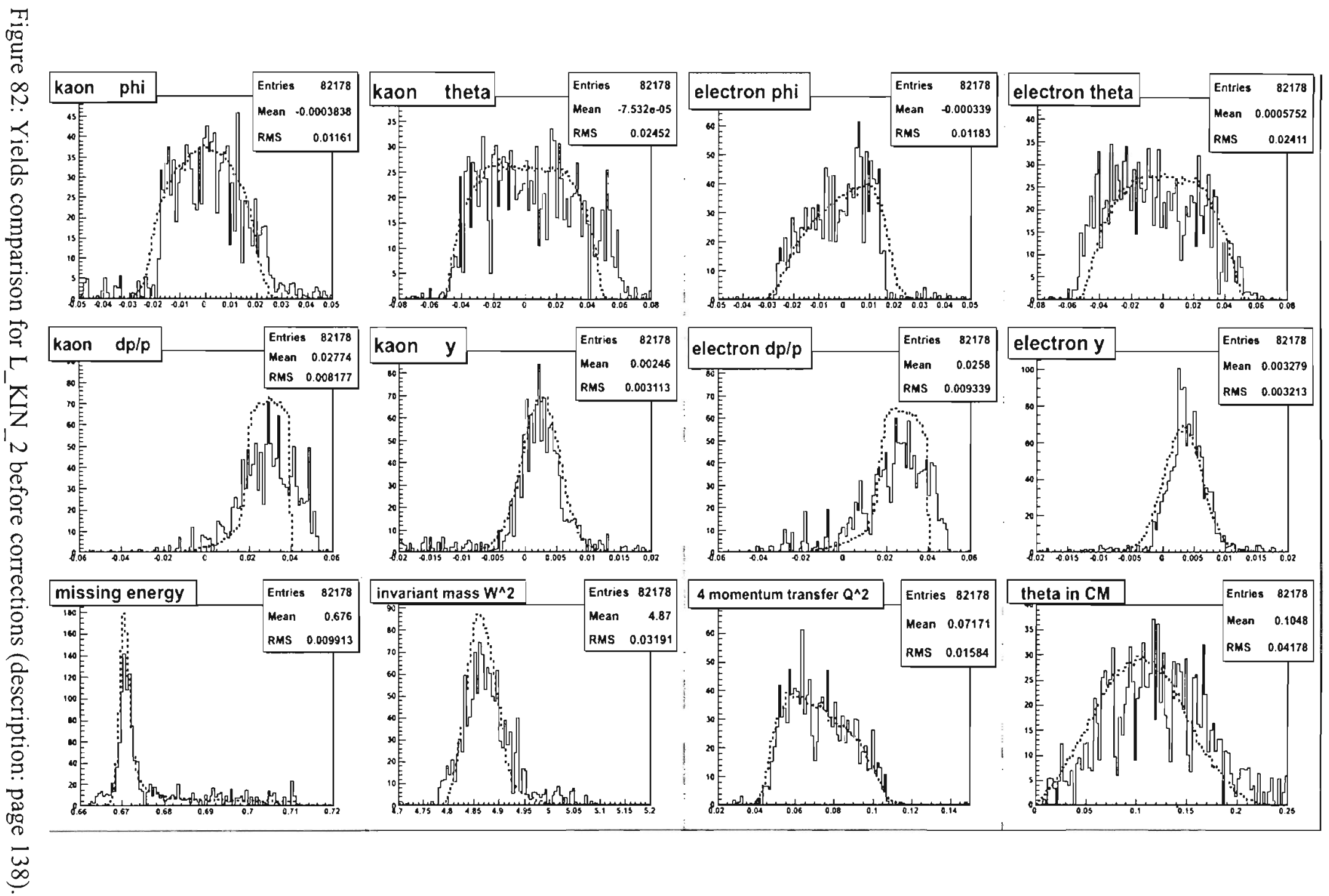




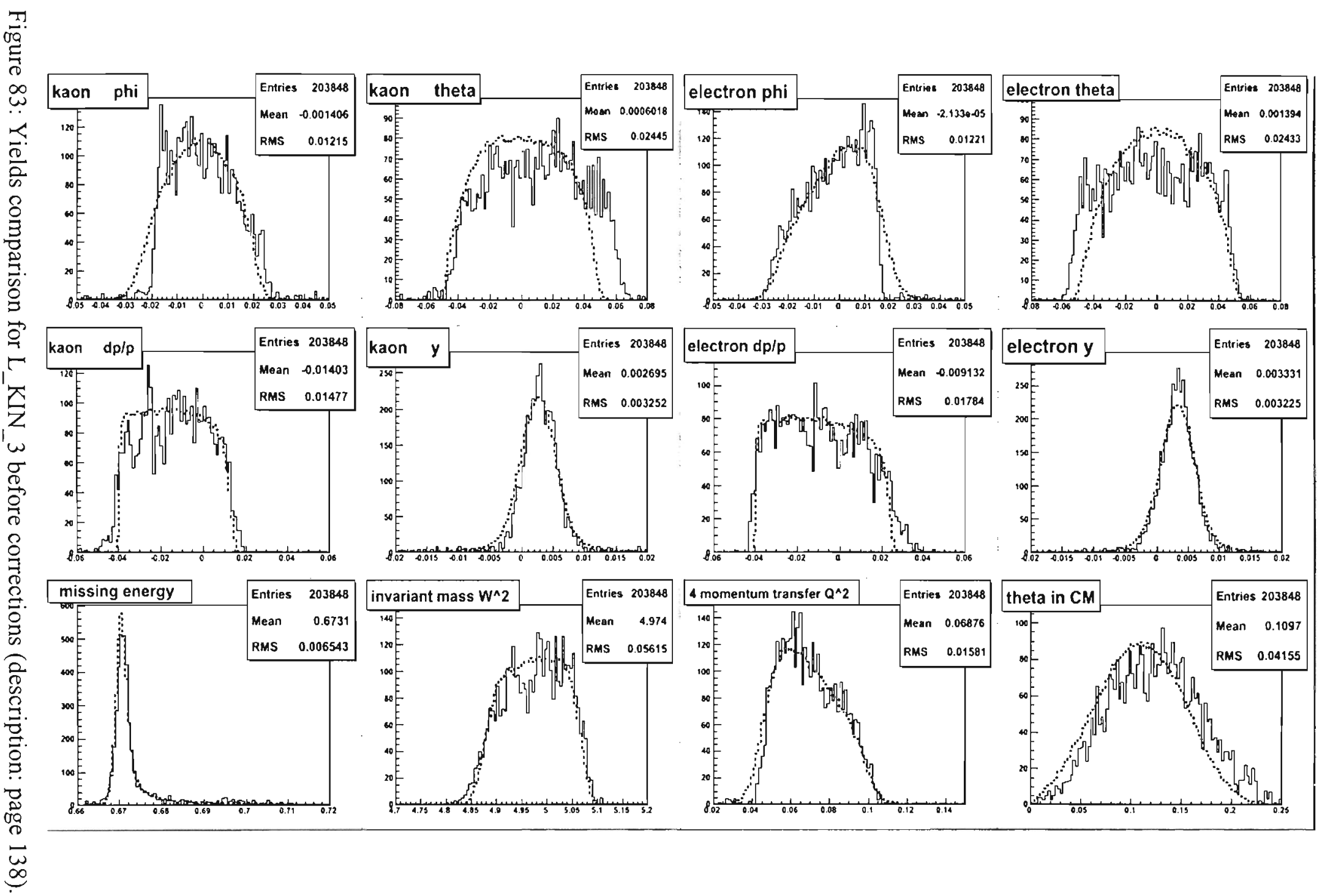




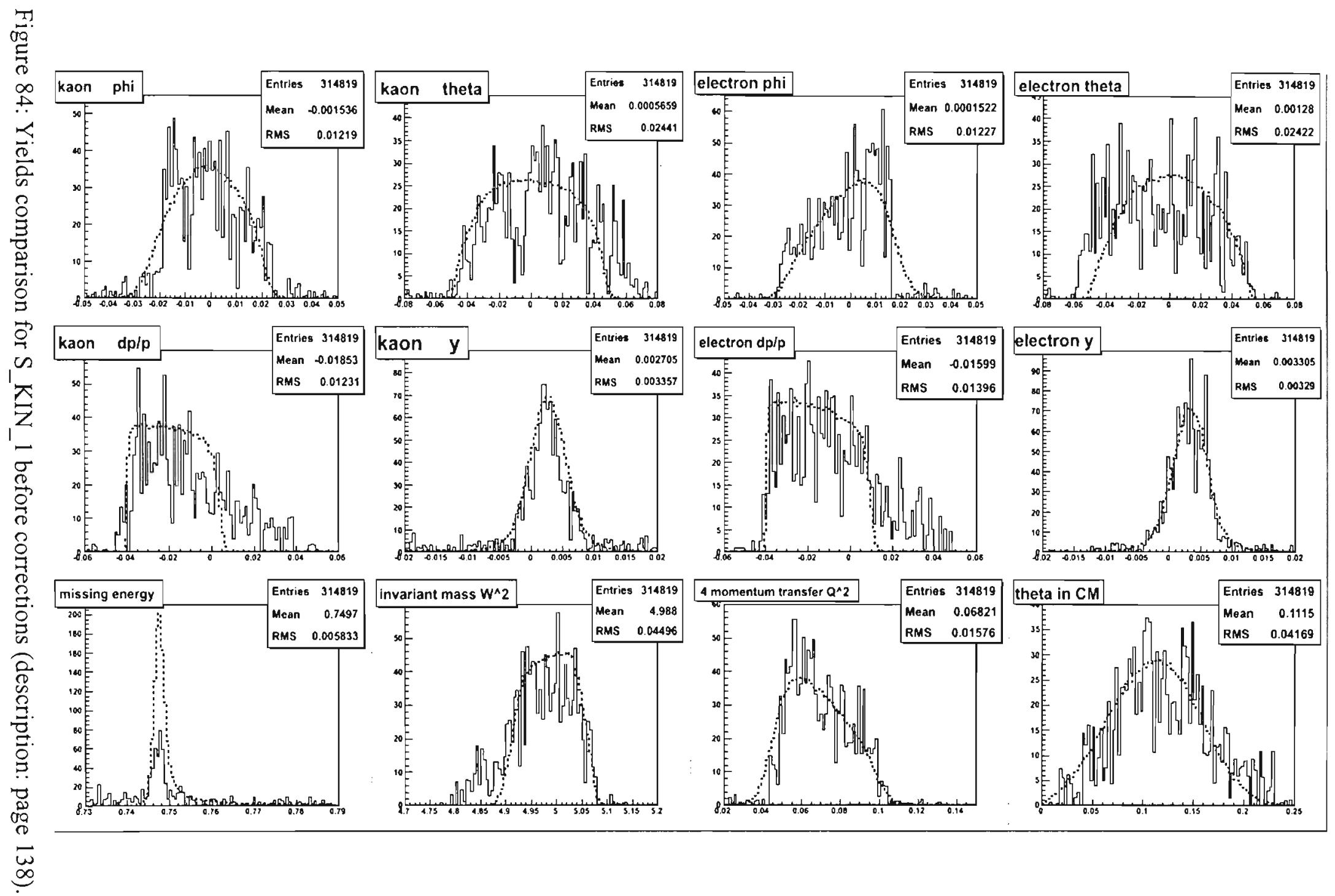




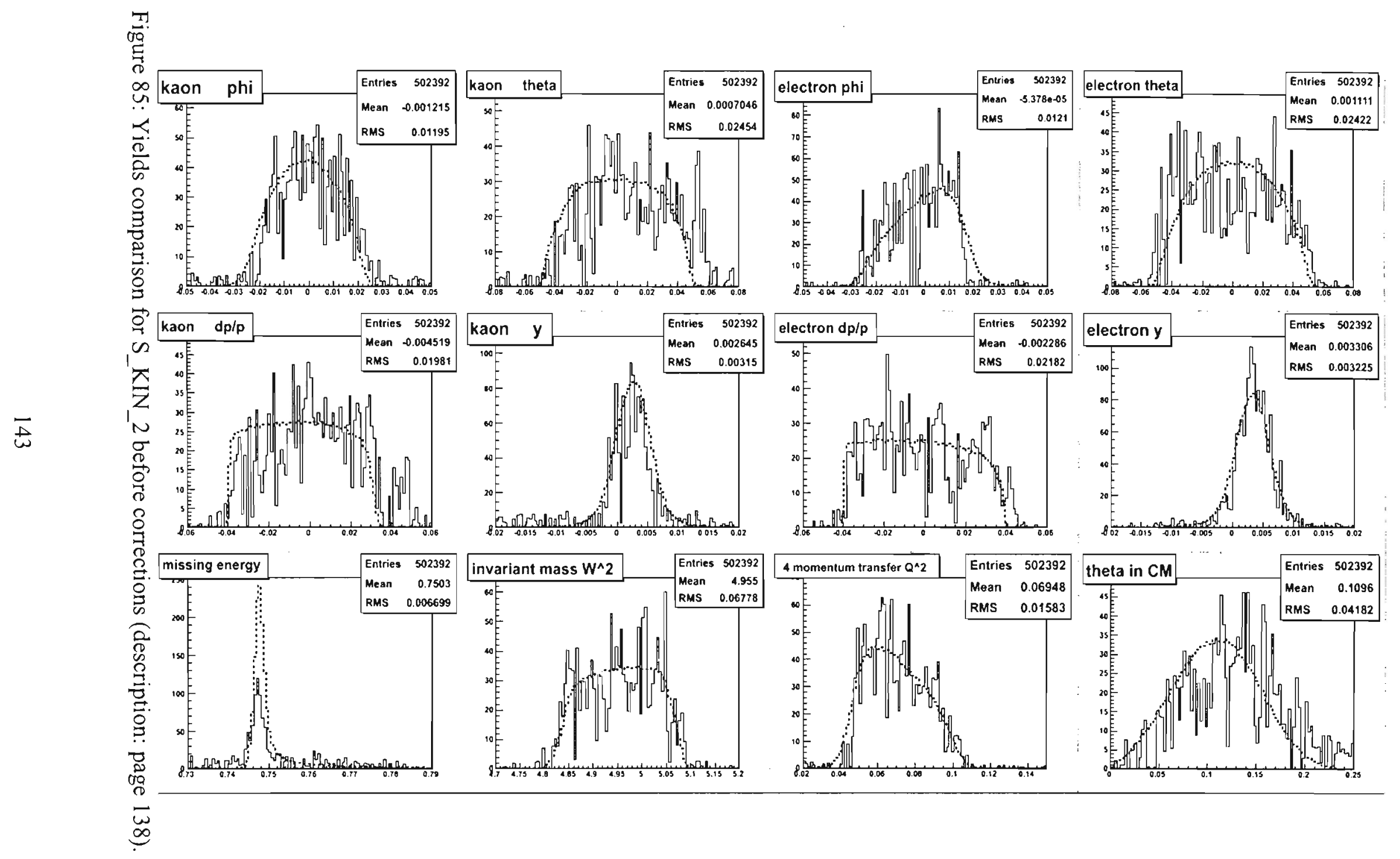


After empirically trying cuts into the angles and in last instance to momentum, it was observed that the following cuts in Table 38 make the most appropriate acceptance variable corrections, providing the best matching between the simulated and real yields.

\begin{tabular}{lllllll}
\hline Kin/particle & $\phi_{K}(\mathrm{rad})$ & $\theta_{K}(\mathrm{rad})$ & $\phi_{e}(\mathrm{rad})$ & $\theta_{e}(\mathrm{rad})$ & $(\delta p / p)_{K}$ & $(\delta p / p)_{e}$ \\
\hline L_KIN_1 & {$[-0.02,0.02]$} & {$[-0.045,0.045]$} & {$[-0.03,0.016]$} & {$[-0.045,0.045]$} & {$[-0.04,0.04]$} & {$[-0.04,0.04]$} \\
& {$[-0.017,0.02]$} & {$[-0.045,0.045]$} & {$[-0.03,0.015]$} & {$[-0.045,0.045]$} & {$[-0.04,0.04]$} & {$[-0.04,0.04]$} \\
L_KIN_2 & & & & & & \\
L_KIN_3 & {$[-0.018,0.02]$} & {$[-0.045,0.045]$} & {$[-0.03,0.016]$} & {$[-0.045,0.045]$} & {$[-0.04,0.04]$} & {$[-0.04,0.04]$} \\
S_KIN_1 & {$[-0.02,0.02]$} & {$[-0.045,0.045]$} & {$[-0.03,0.016]$} & {$[-0.045,0.045]$} & {$[-0.04,0.00]$} & {$[-0.04,0.01]$} \\
S_KIN_2 & {$[-0.017,0.02]$} & {$[-0.045,0.045]$} & {$[-0.03,0.015]$} & {$[-0.045,0.045]$} & {$[-0.04,0.04]$} & {$[-0.04,0.04]$} \\
\hline
\end{tabular}

Table 38: Acceptance variable corrections for all kinematics/particle selections.

It can be seen in Table 38 that the corrections were basically small cuts at the border of the angle ranges and that there was no need to apply cuts on the $\delta p / p$ variable other than the regular cut of $[-0.04,0.04]$ for full acceptance except for the case of $\Sigma^{0}$ particle selected, in which there was a need for a hard cut in the $\delta p / p$.

The cuts shown in the above table were applied to both the MCEEP simulation and in the measurement of the experimental yield and compared again to make sure the corrections were producing well matched yields. The following histograms show the simulated and measured yield normalized. 
종
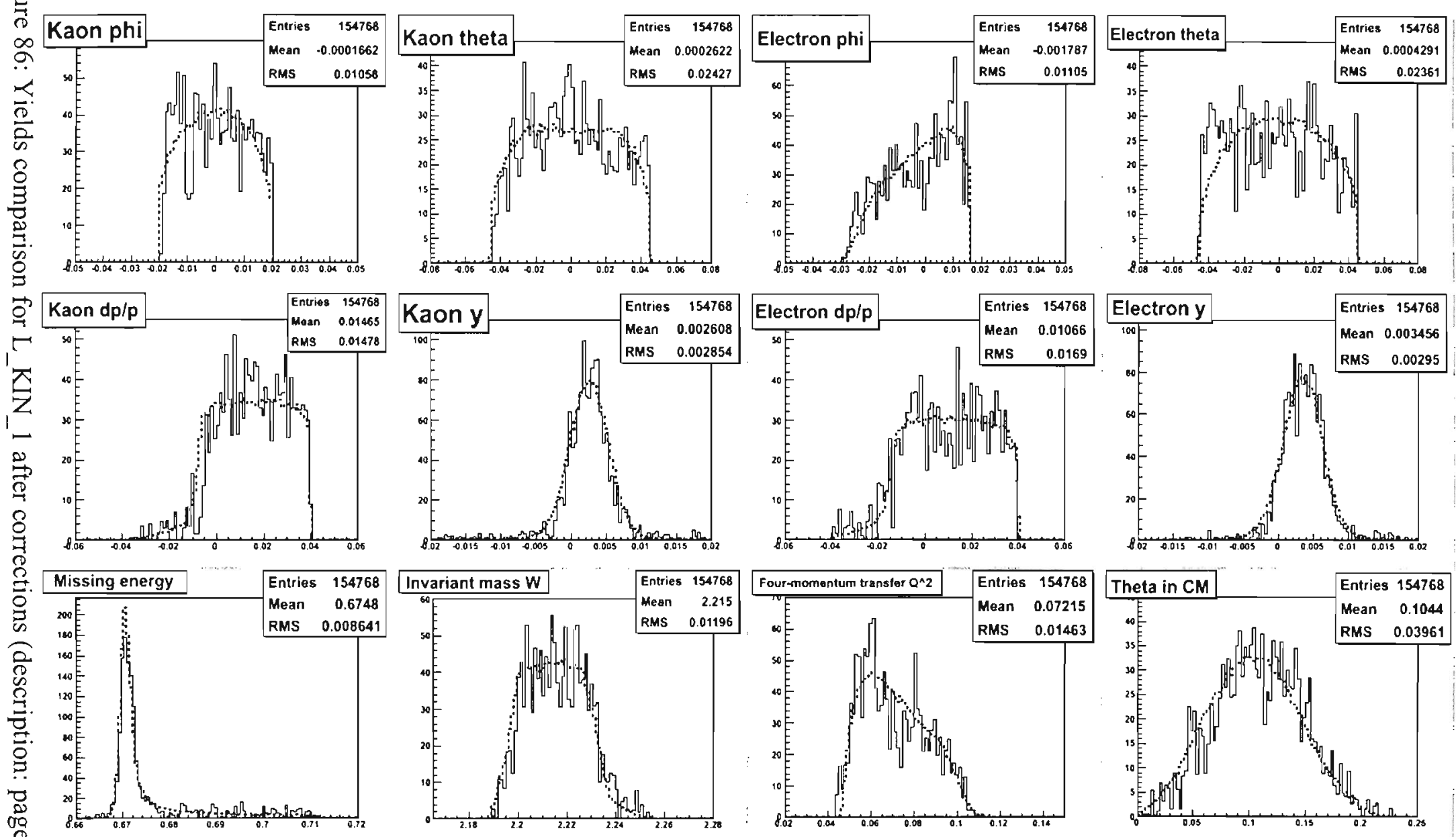

$\underset{\infty}{\infty}$ 
$\stackrel{3}{3}$
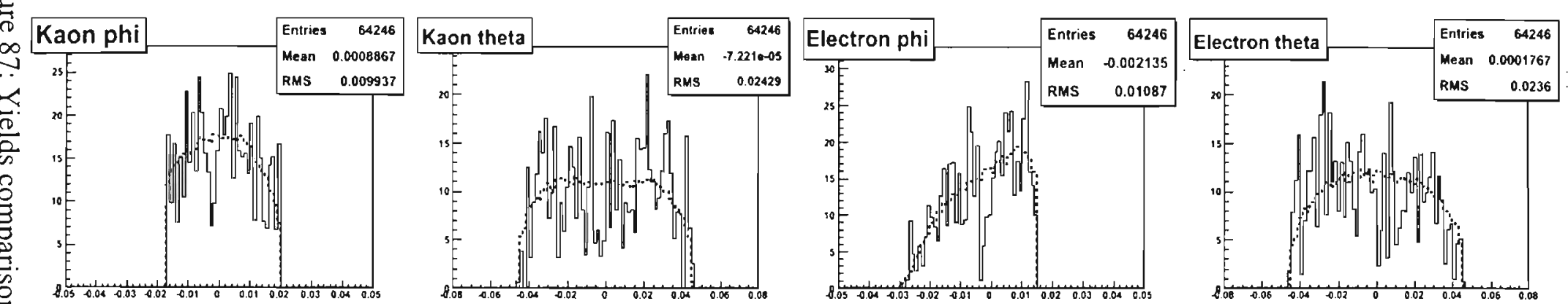

$\frac{F}{6}$
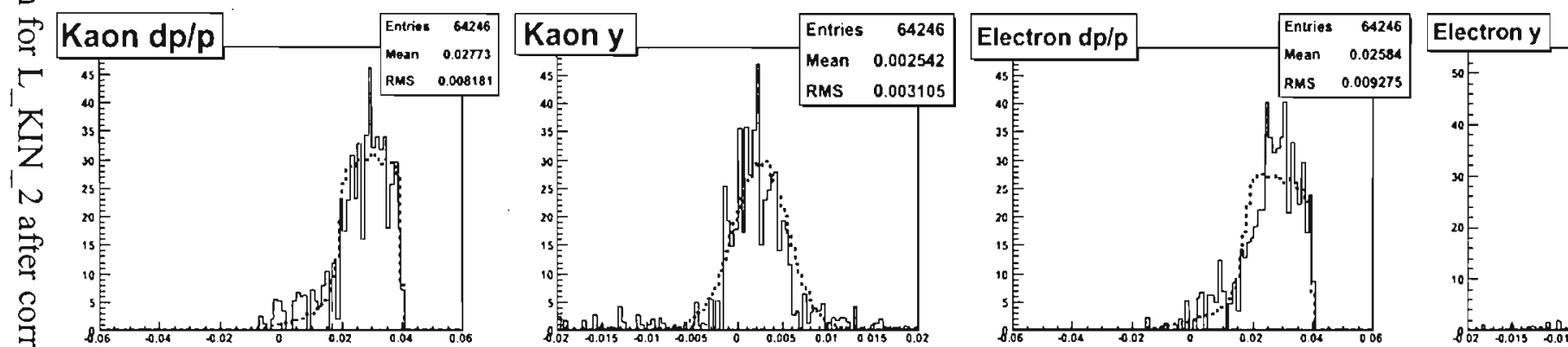

Entries $\quad 64246$
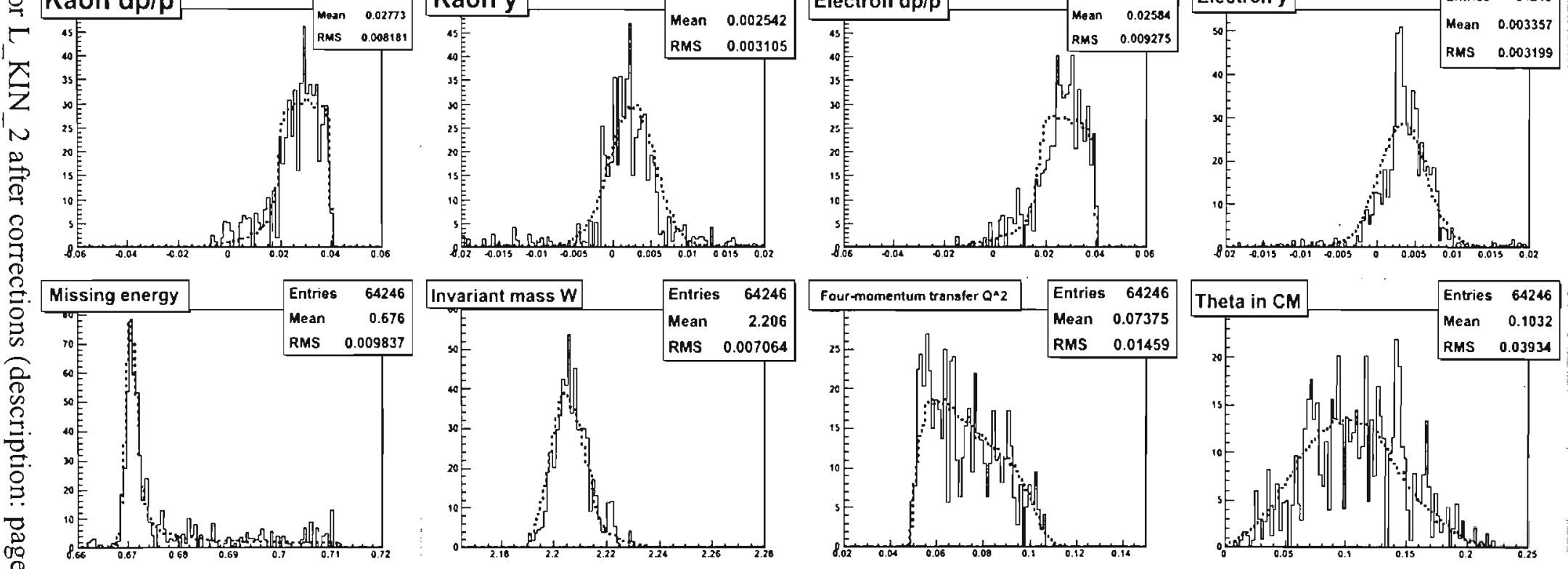

$\underbrace{\infty}$ 


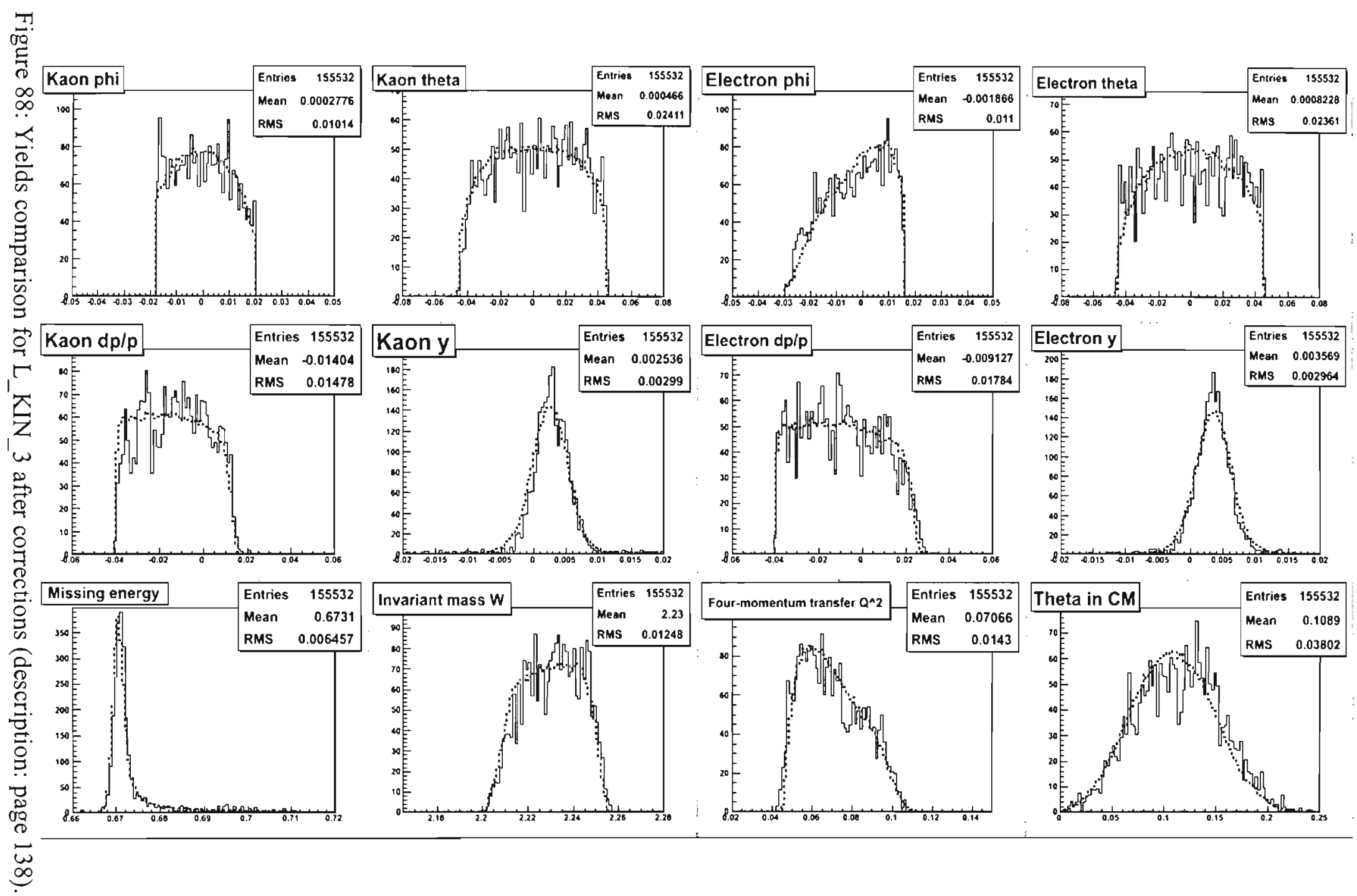




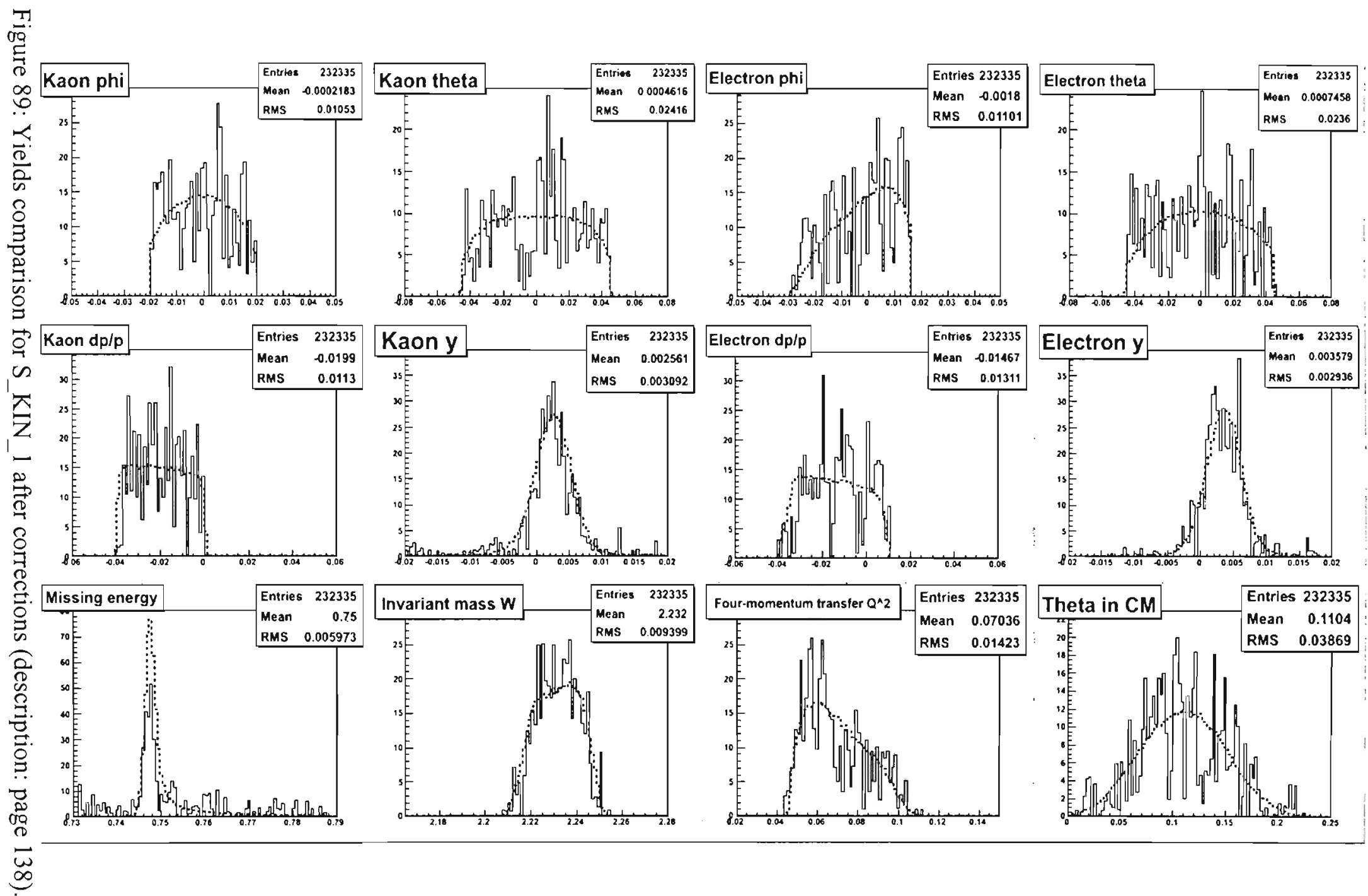




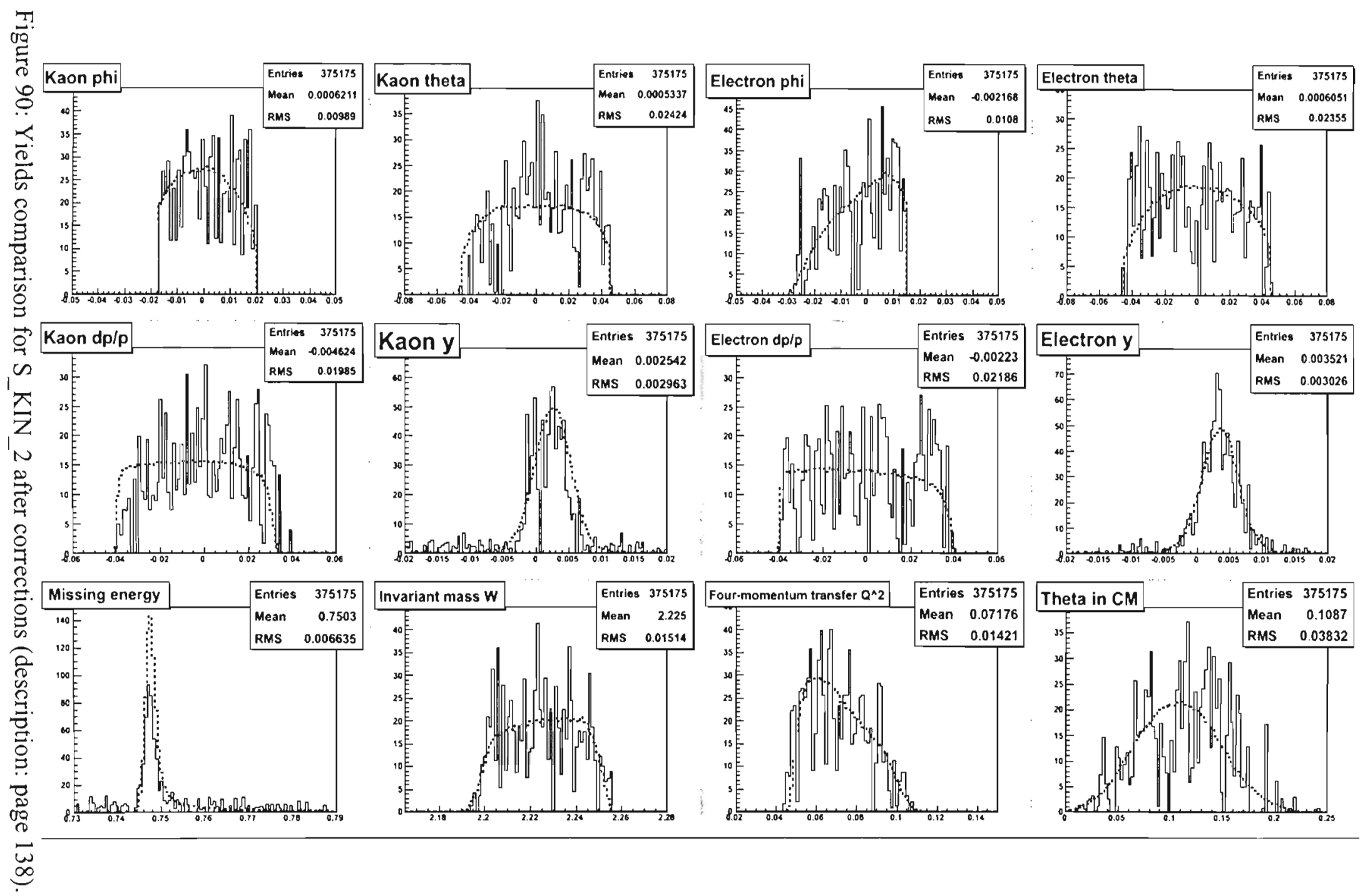


After observing good agreement between the MCEEP yield and the measured yield for the spectrometer variables (with the acceptance correction cuts applied as in Table 38) more histograms for other variables were obtained. Figures 91 to 95 show additional variables described in page 138 (see Figure 2, and Section 1.3).

Another important distribution to consider is the yield around the angle between the reaction plane and the scattering plane ( $\varphi$ in Equation (19)). From the distribution, it can be seen if there are contributions from the interference terms of the cross section in the measured cross section. If the distribution is homogenous around $\varphi$ then the interference terms cancel out in Equation (19). To perform this comparison the Mandelstam variable $t$ is used, and the simulated and real yields are plotted on the reconstructed variable $t \cos (\varphi)$ vs. $t \sin (\varphi)(\mathrm{GeV})^{2}$. The results are shown in Figures 96100. The distributions are not homogeneous and they have a semi "donut" shape and the MCEEP yields are mnormalized to match data yield. The MCEEP simulated yield matched very well with the measured yield. For a description of the variables shown in Figures $96-100$ see page 138 . 


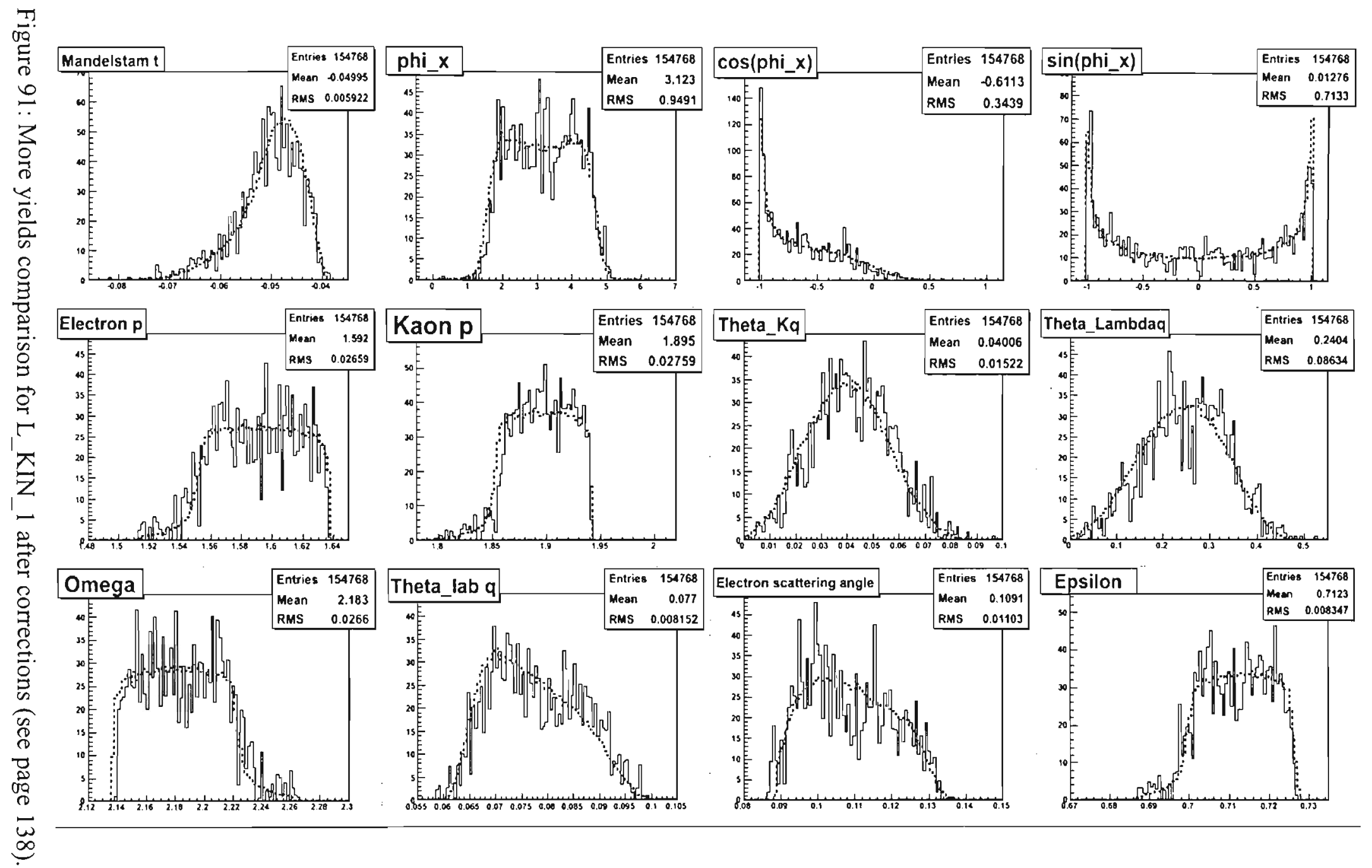




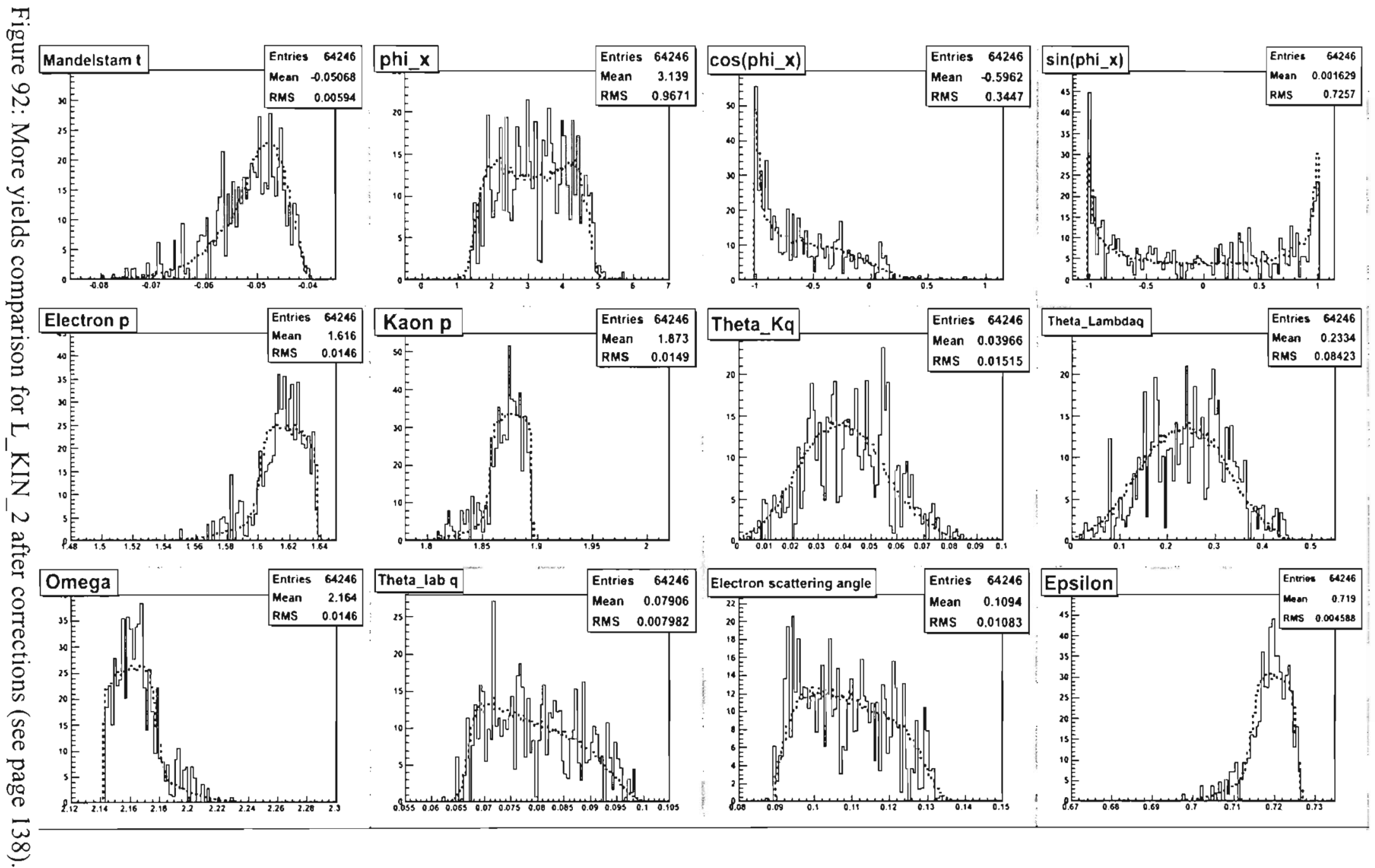




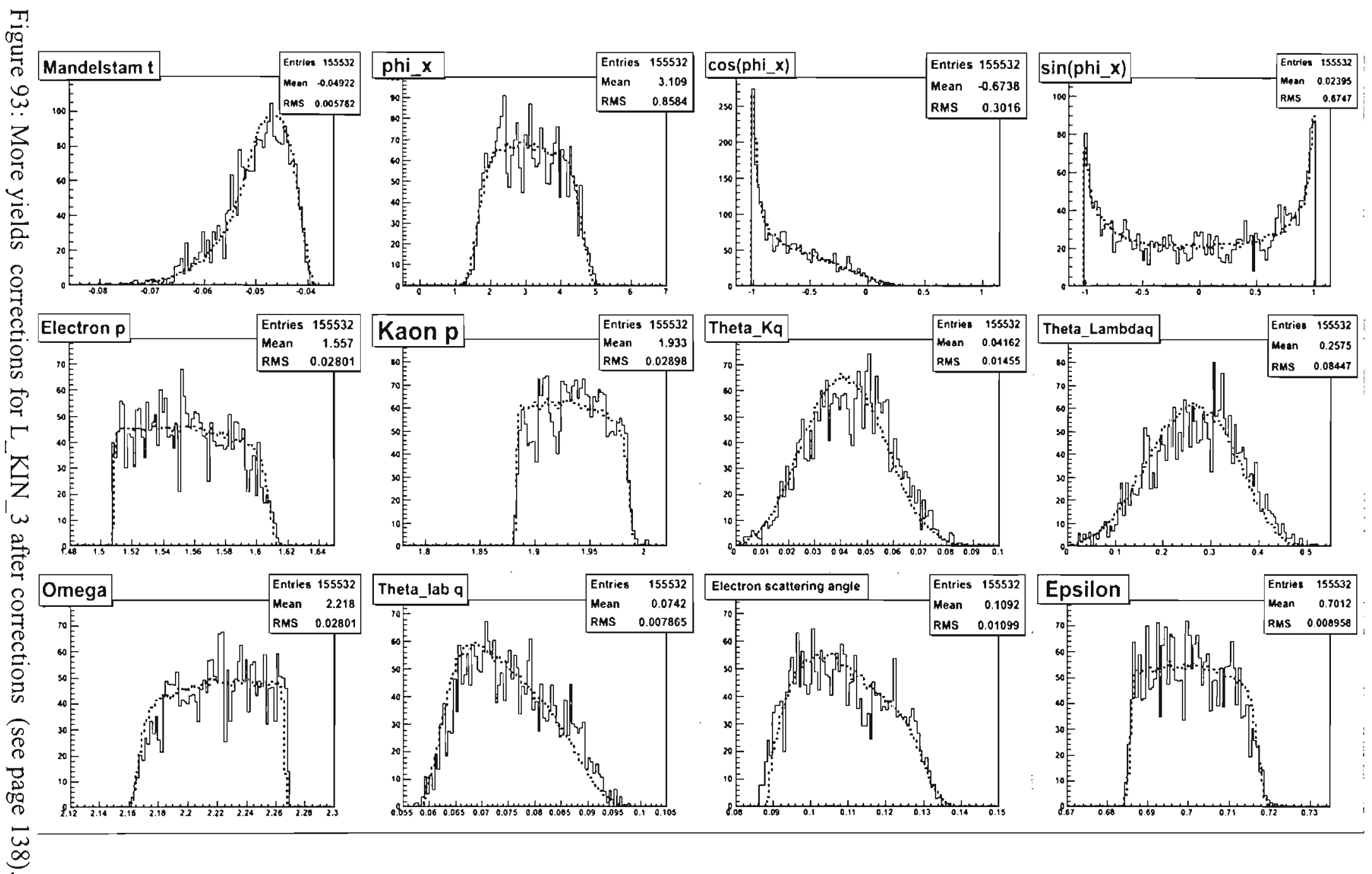




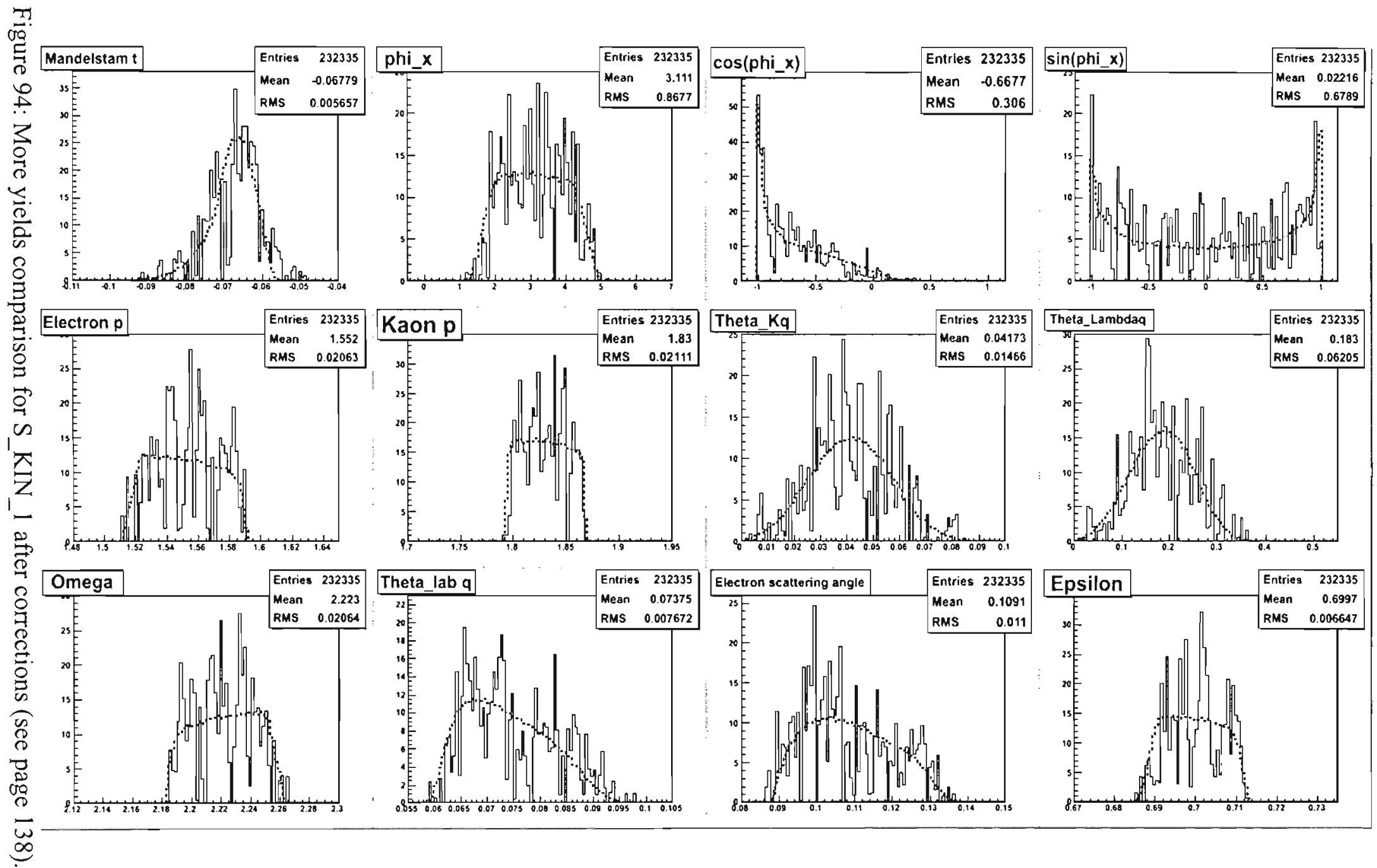




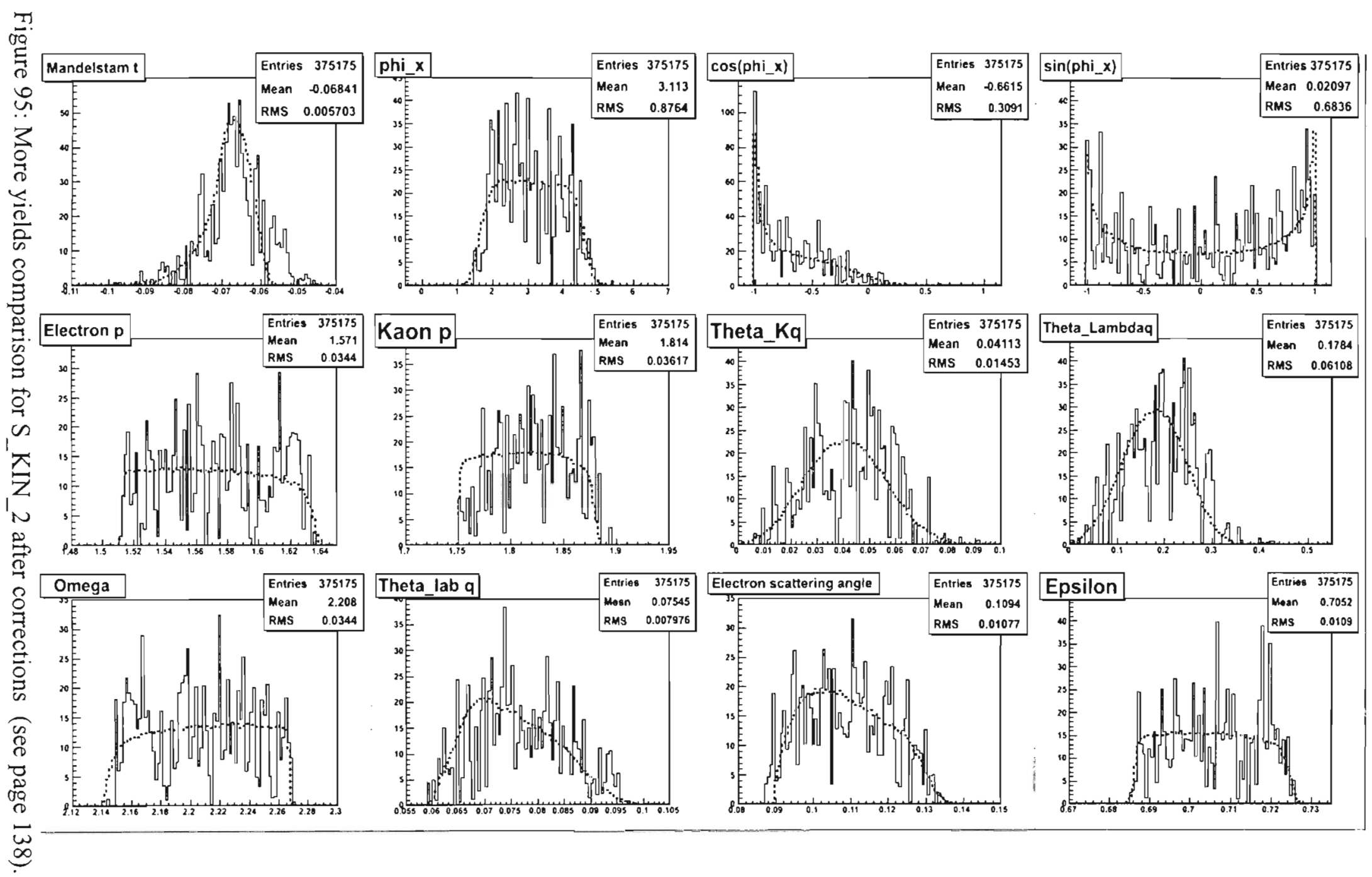




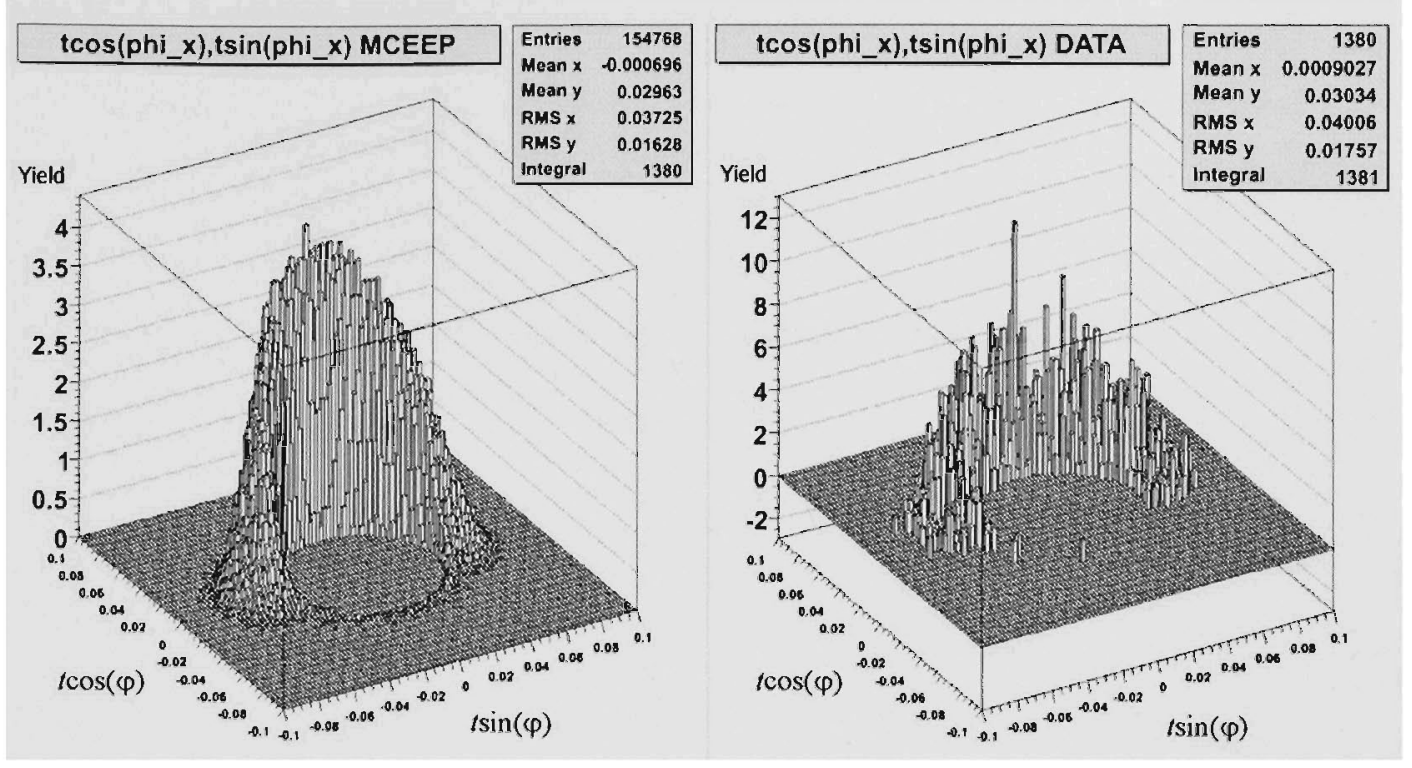

Figure 96: Yields distributions on $\varphi$ for L_KIN_1 after corrections. For a description see page 138 and Section 1.3.

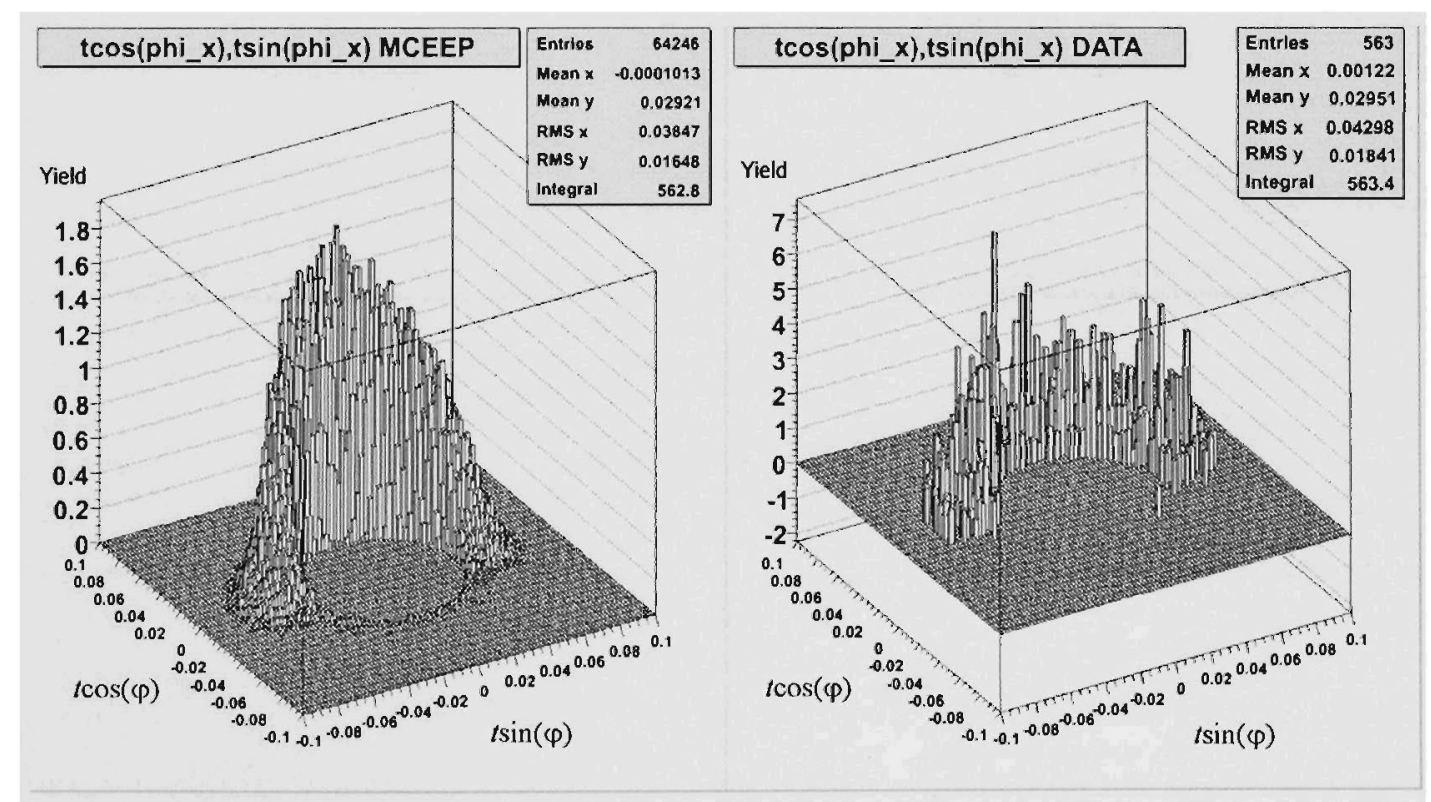

Figure 97: Yields distributions on $\varphi$ for L_KIN_2 after corrections. For a description see page 138 and Section 1.3. 


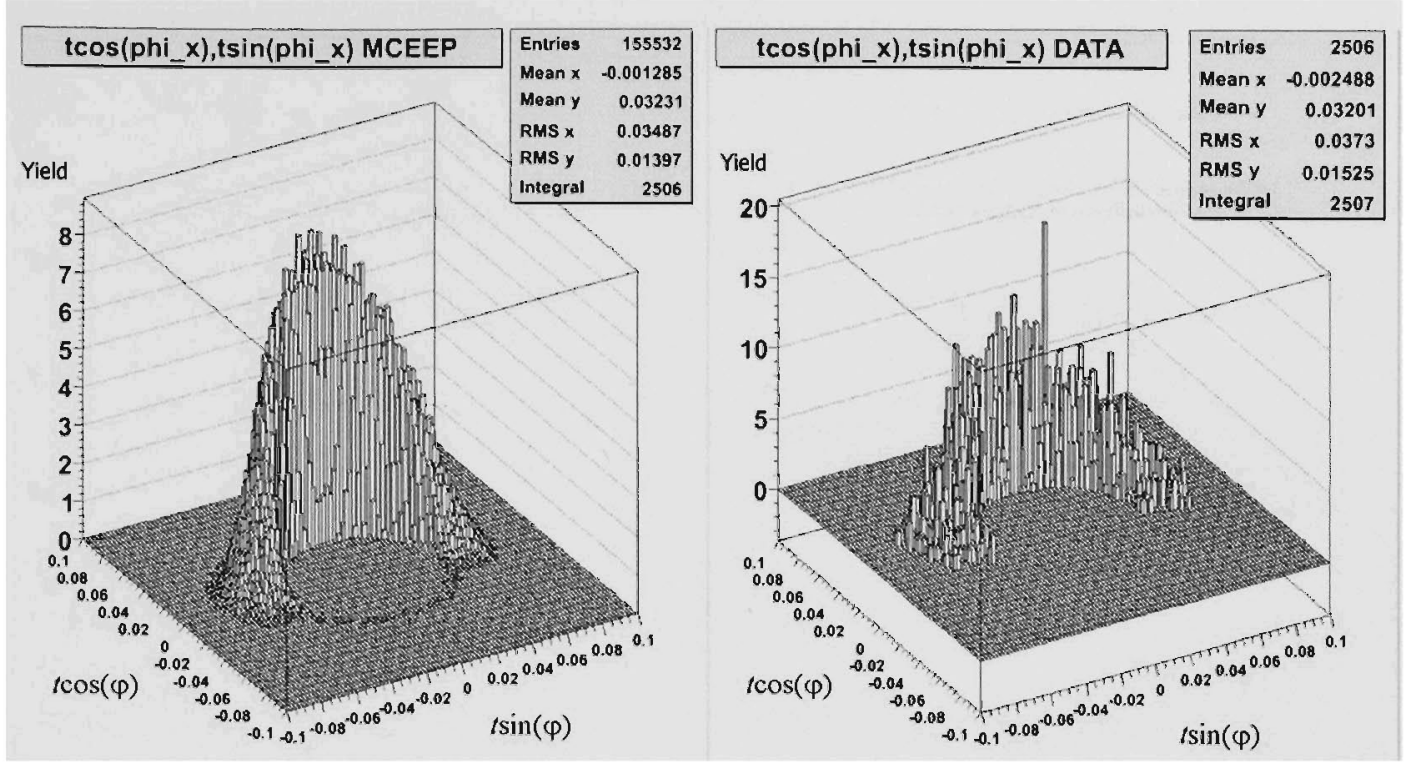

Figure 98: Yields distributions on $\varphi$ for $L_{-}$KIN_3 after corrections. For a description see page 138 and Section 1.3.

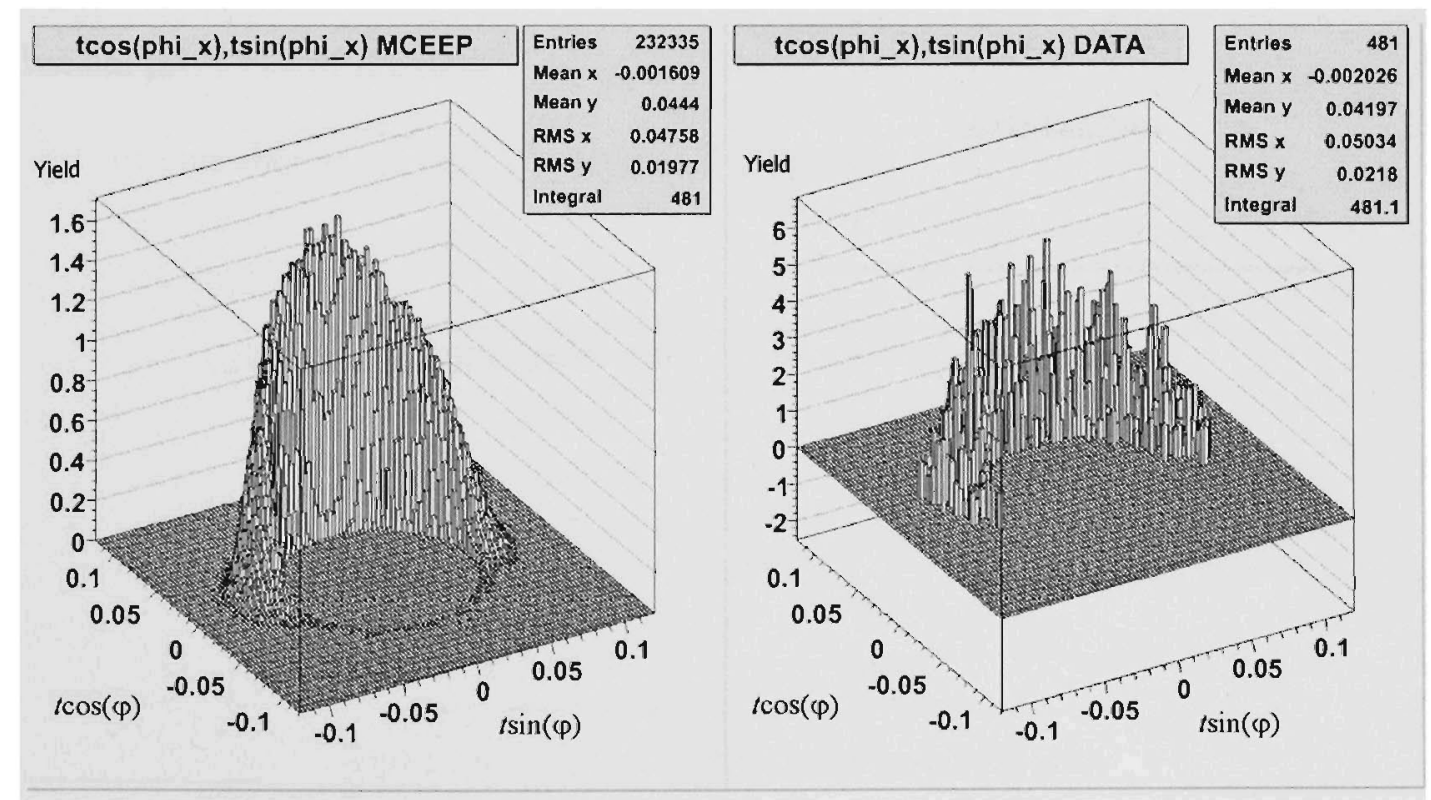

Figure 99: Yields distributions on $\varphi$ for $S_{-}$KIN_l after corrections. For a description see page 138 and Section 1.3. 


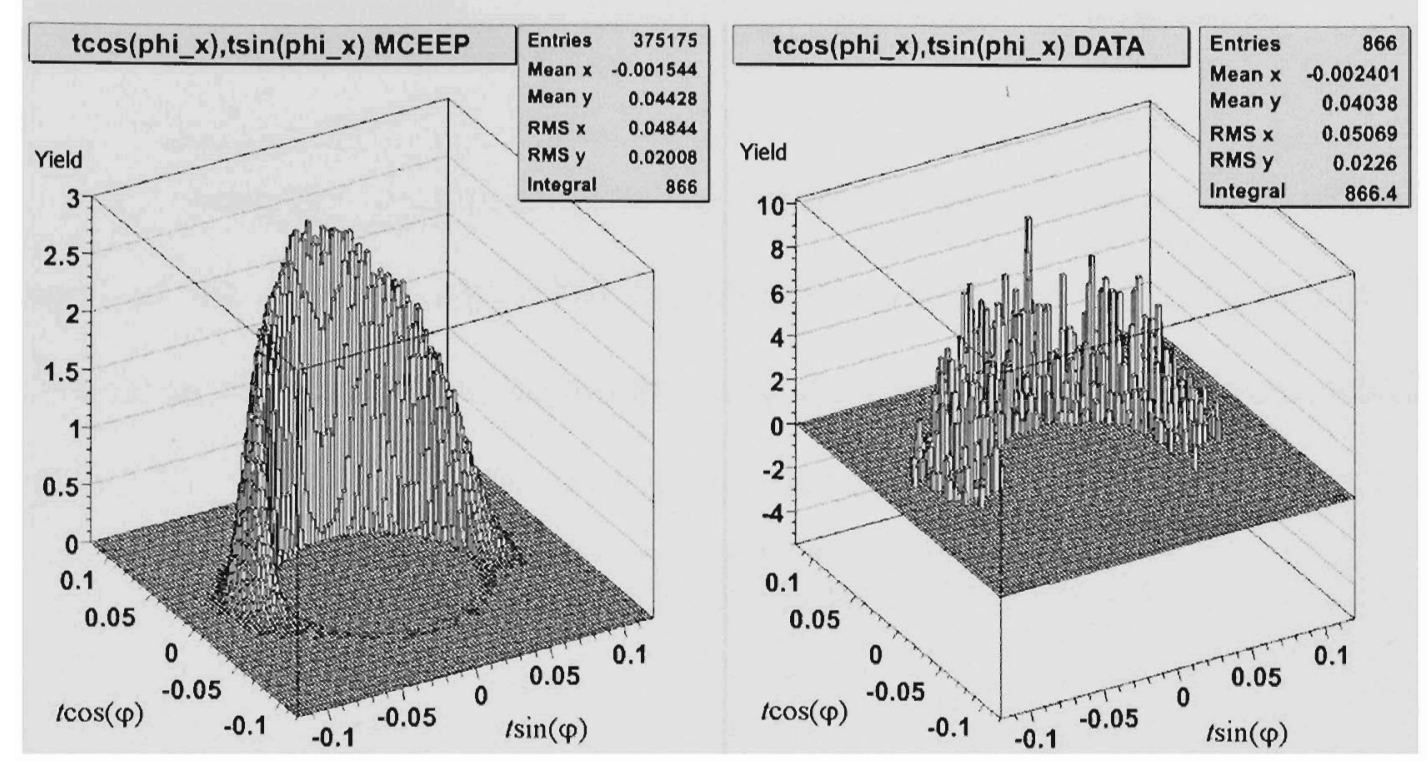

Figure 100: Yields distributions on $\varphi$ for S_KIN_2 after corrections. For a description see page 138 and Section 1.3.

A good agreement is observed for the $\Lambda$ channel. However, the quality of the agreement for the $\Sigma^{0}$ channel is harder to discern as a result of the relatively poor statistical precision of the data.

The number of $\Lambda$ and $\Sigma^{0}$ particles found were:

\begin{tabular}{cc}
\hline Kinematics/particle & Number of hyperons \\
\hline L_KIN_l $_{-}$ & $1380 \Lambda$ \\
L_KIN_2 $_{-}$ & $563 \Lambda$ \\
L_KIN_3 & $2506 \Lambda$ \\
S_KIN_1 & $481 \Sigma^{0}$ \\
S_KIN_2 & $866 \Sigma^{0}$ \\
\hline
\end{tabular}

Table 39: Total measured experimental yield. 


\subsection{Cross-section measurements}

To obtain the measurements of the cross sections it is necessary to know the integral of the electron charge and the number of potential scattering centers. The measurement of the charge is performed using the beam current monitors described in Section 2.3.1. Table 40 shows the total charge used in E94-107 ( cryotarget) for each run.

\begin{tabular}{|c|c|c|c|}
\hline Run & Charge $(\mathrm{C})$ & Uncertainty (C) & Uncertainty $(\%)$ \\
\hline 2485 & 0.0503 & 0.0002 & 0.40 \\
\hline 2486 & 0.0499 & 0.0002 & 0.40 \\
\hline 2487 & 0.05 & 0.0003 & 0.60 \\
\hline 2488 & 0.0398 & 0.0003 & 0.75 \\
\hline 2489 & 0.0399 & 0.0001 & 0.25 \\
\hline 2490 & 0.0403 & 0.0002 & 0.50 \\
\hline 2491 & 0.0391 & 0.0002 & 0.51 \\
\hline 2492 & 0.0397 & 0.0002 & 0.50 \\
\hline 2496 & 0.0411 & 0.0003 & 0.73 \\
\hline 2497 & 0.0428 & 0.0003 & 0.70 \\
\hline 2498 & 0.043 & 0.0004 & 0.93 \\
\hline 2499 & 0.0425 & 0.0004 & 0.94 \\
\hline 2500 & 0.0428 & 0.0004 & 0.93 \\
\hline 2501 & 0.0374 & 0.0003 & 0.80 \\
\hline 2503 & 0 & & \\
\hline 2504 & 0.0425 & 0.0004 & 0.94 \\
\hline 2505 & 0.0428 & 0.0004 & 0.93 \\
\hline 2506 & 0.0171 & 0.0002 & 1.17 \\
\hline 2507 & 0.0426 & 0.0005 & 1.17 \\
\hline 2508 & 0.0426 & 0.0004 & 0.94 \\
\hline 2509 & 0.0471 & 0.0004 & 0.85 \\
\hline 2510 & 0.047 & 0.0004 & 0.85 \\
\hline 2511 & 0.0467 & 0.0004 & 0.86 \\
\hline 2512 & 0.047 & 0.0004 & 0.85 \\
\hline 2513 & 0.0473 & 0.0004 & 0.85 \\
\hline 2514 & 0.0471 & 0.0004 & 0.85 \\
\hline 2515 & 0.0471 & 0.0004 & 0.85 \\
\hline 2516 & 0.0461 & 0.0004 & 0.87 \\
\hline 2517 & 0.0469 & 0.0004 & 0.85 \\
\hline 2518 & 0.0467 & 0.0004 & 0.86 \\
\hline 2519 & 0.0468 & 0.0005 & 1.07 \\
\hline 2520 & 0.0474 & 0.0004 & 0.84 \\
\hline 2521 & 0.0473 & 0.0003 & 0.63 \\
\hline
\end{tabular}

Table 40: Charges and uncertainties in E94-107 from BCM measurements. 


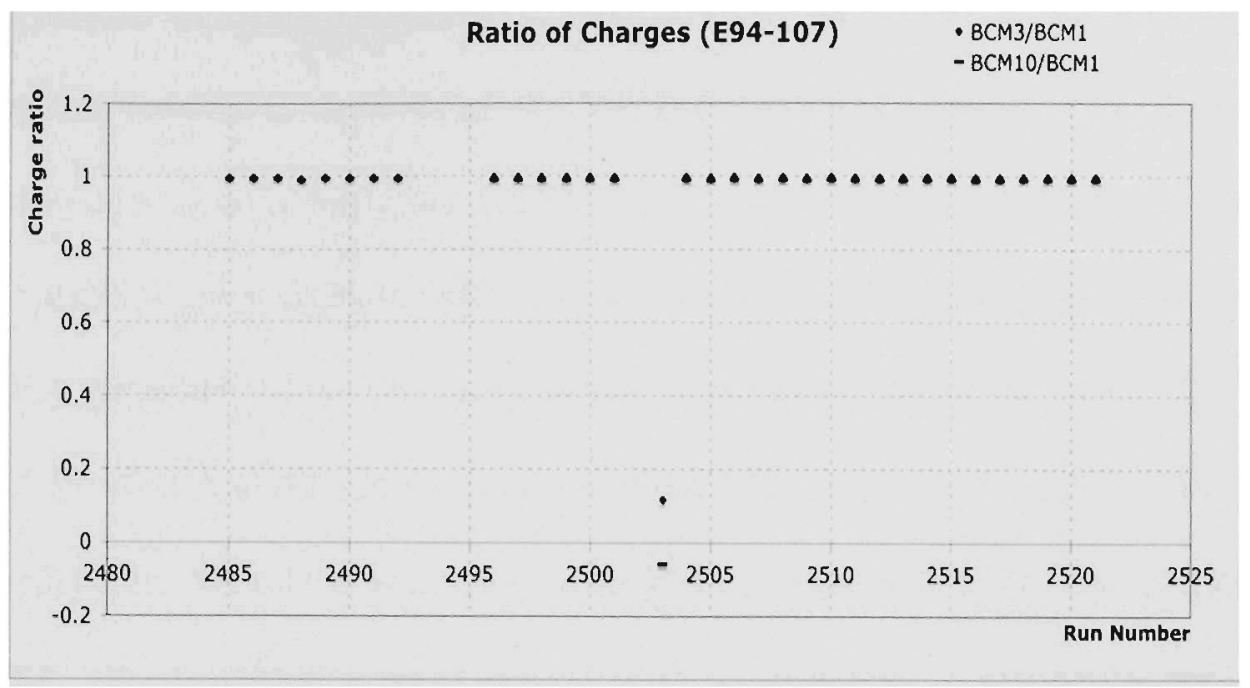

Figure 101: Charge ratio among different BCM.

The uncertainties in Table 40 were obtained by comparing the three BCMs during the experiment and, therefore, represent a systematic uncertainty (for details see Section 4.9).

Former JLab graduate student, J. Cha [77] did a fit to the experimental data obtained at JLab on $\Lambda$ electroproduction in the late 90's (Experiment E91-016), from which later, a program was developed by the hypernuclear collaboration to find out what the cross section should be for different values of $Q^{2}, W$ and $\theta_{C M}$ and by including the scaling factors described in Section 4.6.3. The program is called KFIT3s.f and by introducing the beam energy value $(E)$, momentum transfer squared $\left(Q^{2}\right)$, invariant mass $(W)$, left spectrometer momentum $\left(p_{K}\right)$ and the angle between the virtual photon and the kaon particle $\left(\theta_{K}\right)$ (see Section 1.3) gives the corresponding cross section on the basis of a fit of the available experimental data [77]. See Appendix H for details of KFIT3s.f. 
Instead of using Equation (15) directly for the cross-section measurements, a different approach was used. Since the MCEEP simulation was used for the acceptance correction cuts by comparing the MCEEP yield to the measured yield, the average values of the variables taken from the MCEEP output summary were used as input to KFITs3.f to get the expected cross sections (cross-section values from Cha's fit [77]). Then, the ratio of the corrected measured yield to the MCEEP yield and the ratio of the measured cross section in E94-107 to the expected cross section from KFIT3s.f are considered to be the same. The measured yield is corrected by a correction factor, where the correction factor is calculated as the inverse of the product of all the detectors efficiencies and inefficiencies [68]. Therefore, the equations are:

$$
\frac{d \sigma_{\mathrm{exp}}}{d \Omega}=Y_{\text {ratio }} \frac{d \sigma_{\text {expected }}}{d \Omega}
$$

with,

$$
\begin{aligned}
& Y_{\text {ratio }}=\frac{Y_{\exp }^{\text {corrected }}}{Y_{M C E E P}}, \\
& Y_{\exp }^{\text {corrected }}=Y_{\exp } F_{\text {correction }}, \\
& F_{\text {correction }}=\frac{1}{E_{V D C} \cdot E_{S C I N T} \cdot I_{A 1} \cdot E_{A 2} \cdot E_{C O_{2}} \cdot L \cdot A},
\end{aligned}
$$


where $E_{V D C}, E_{S C I N T}, E_{A 2}, E_{C O_{2}}$ are detector efficiencies, $I_{A 1}$ is the A1 inefficiency, $L$ is the livetime factor and $A$ is the absorption factor.

It is also typical to express the yield as the yield per unit of charge, which can be simply obtained by just dividing the yield obtained, either from the simulation or from the experiment, by the total charge (Table 40) for each kinematics (Table 15). However, the ratio in (57) remains the same. The experimental yields are in Table 39.

The correction factors are shown in Table 41.

\begin{tabular}{ccccccccc}
\hline $\begin{array}{c}\text { Kinematics } \\
\text { particle }\end{array}$ & $\begin{array}{c}\text { Correction } \\
\text { Factor }\end{array}$ & VDC & A1 & A2 & CO2 & Deadtime & Scint_eff & Absorp. \\
\hline L_KIN_1 & 1.8751 & 1.3577 & 1.0978 & 1.0042 & 1.0190 & 1.1089 & 1.06900 & 1.0372 \\
L_KIN_2 & 2.0931 & 1.4406 & 1.1074 & 1.0064 & 1.0189 & 1.1497 & 1.07200 & 1.0381 \\
L_KIN_3 & 2.0709 & 1.4285 & 1.1208 & 1.0063 & 1.0272 & 1.1299 & 1.06950 & 1.0354 \\
S_KIN_1 & 1.7373 & 1.3577 & 1.0308 & 0.9909 & 1.0190 & 1.1089 & 1.06900 & 1.0372 \\
S_KIN_2 & 2.1335 & 1.4406 & 1.1450 & 0.9921 & 1.0189 & 1.1497 & 1.07200 & 1.0381 \\
\hline
\end{tabular}

Table 41: Correction factors for all kinematics/particles.

In the case of the VDC, deadtime, and scintillator correction factors, it is important to remark that because the analyses were performed run by run, the combined efficiency (or correction factor as the inverse) is previously weighted by the charge of each run by the equation:

$$
E=\frac{\sum Q_{i} E_{i}}{\sum Q_{i}}
$$


Table 42 shows the input values to the KFITs3.f program that were taken from the MCEEP summary output (Appendix I). In Table 42, the average values for the beam energy value $(E)$, momentum transfer squared $\left(Q^{2}\right)$, invariant mass $(W)$, left spectrometer momentum $\left(p_{K}\right)$ and the angle between the virtual photon and the kaon particle $\left(\theta_{K}\right)$ are shown.

\begin{tabular}{cccccc}
\hline kinematics/particle & $\begin{array}{c}E \\
(\mathrm{GeV})\end{array}$ & $\begin{array}{c}Q^{2} \\
(\mathrm{GeV} / \mathrm{c})^{2}\end{array}$ & $\begin{array}{c}W \\
(\mathrm{GeV})\end{array}$ & $\begin{array}{c}p_{K} \\
(\mathrm{GeV} / \mathrm{c})\end{array}$ & $\begin{array}{c}\theta_{K} \\
\text { (degrees) }\end{array}$ \\
\hline L_KIN_1 & 3.7758 & 0.0750 & 2.2140 & 1.8934 & 2.2420 \\
L_KIN_2 & 3.7807 & 0.0770 & 2.2060 & 1.8721 & 2.2230 \\
L_KIN_3 & 3.7758 & 0.0740 & 2.2290 & 1.9319 & 2.3300 \\
S_KIN_1 & 3.7758 & 0.0730 & 2.2320 & 1.8293 & 2.3340 \\
S_KIN_2 & 3.7807 & 0.0750 & 2.2250 & 1.8122 & 2.3040 \\
\hline
\end{tabular}

Table 42: KFIT3s.f input values from the MCEEP simulation.

In Table 43, the output values (expected cross sections) from KFIT3s.f are shown after entering input values shown in Table 42.

\begin{tabular}{cc}
\hline kinematics/particle & $\begin{array}{c}\text { Expected C.S. } \\
(\mu \mathrm{B} / \mathrm{sr})\end{array}$ \\
\hline L_KIN_1 & 0.5800 \\
L_KIN_2 & 0.5829 \\
L_KIN_3 & 0.5728 \\
S_KIN_1 & 0.5424 \\
S_KIN_2 & 0.5449 \\
\hline
\end{tabular}

Table 43: Expected cross sections for all kinematics/particle selections (from KFIT3s.f). 
The corrected experimental yields are obtained by multiplying the experimental yields shown in Table 39 by the correction factors shown in Table 41. The simulated and corrected experimetal yields, as well as the ratios (see Equation (57)), are shown in Table 44.

\begin{tabular}{cccc}
\hline kinematics/particle & MCEEP yield & $\begin{array}{c}\text { Experimental Yield } \\
\text { (corrected) }\end{array}$ & Ratio \\
\hline L_KIN_1 & 2597 & 2588 & 0.9963 \\
L_KIN_2 & 1299 & 1178 & 0.9068 \\
L_KIN_3 & 4710 & 5190 & 1.1020 \\
S_KIN_1 & 1836 & 836 & 0.4552 \\
S_KIN_2 & 3698 & 1848 & 0.4996 \\
\hline
\end{tabular}

Table 44: Yields and ratios for all kinematics/particle selections.

From Tables 43, 44, and Equation (56) the measured experimental cross sections were obtained as shown in Table 45 .

\begin{tabular}{ccccc}
\hline kinematics/particle & $\begin{array}{c}\text { Measured Cross Section } \\
(\mu \mathrm{B} / \mathrm{sr})\end{array}$ & $\begin{array}{c}\text { Average } \\
\text { experimental } \\
Q^{2}(\mathrm{GeV} / \mathrm{c})^{2}\end{array}$ & $\begin{array}{c}\text { Average } \\
\text { experimental } \\
W(\mathrm{GeV})\end{array}$ & $\begin{array}{c}\text { Average } \\
\text { experimental } \\
\theta_{C M}(\mathrm{rad})\end{array}$ \\
\hline L_KIN_1 & 0.5779 & 0.0710 & 2.2160 & 0.1089 \\
L_KIN_2 & 0.5286 & 0.0719 & 2.2080 & 0.1092 \\
L_KIN_3 & 0.6312 & 0.0699 & 2.2310 & 0.1134 \\
S_KIN_1 & 0.2469 & 0.0691 & 2.2320 & 0.1108 \\
S_KIN_2 & 0.2723 & 0.0711 & 2.2250 & 0.1145 \\
\hline
\end{tabular}

Table 45: Experimental cross sections in E94-107. Experimental average variable values are listed. Uncertainties discussed in Section 4.9. 
When the analysis is performed with the inclusion of the RICH detector, it causes the statistics to decrease. However, after correcting for RICH efficiency, the cross sections were similar, but also using the RICH produced a larger statistical uncertainty. The RICH performs very well in reducing the background, something crucial for resolving hypernuclear energy states with different targets or nuclear core states. In this analysis since the $\Lambda$ and $\Sigma^{0}$ peaks are quite evident above the background the $\mathrm{RICH}$ is not really necessary.

\subsection{Uncertainty measurements}

The origin of the many of the uncertainties in this work is statistical in nature. However, the uncertainties in the cross-section measurements produced when analyzing the efficiencies, inefficiencies, or correcting factors of the detectors, absorption and deadtime, called systematic uncertainties, are taken separately from the statistical ones that comes from the yields themselves, because later, when the data are binned in different variables to study the cross-section measurement trends, the statistical uncertainties depend on the number of counts for each bin while the systematic uncertainties remain the same. The efficiencies of the different detectors follow binomial or Poisson distributions because of the dependence between the numerator and denominator of the general detector efficiency $\left(\varepsilon_{d e t}\right)$ formula:

$$
\varepsilon_{\mathrm{det}}=\frac{N_{\mathrm{det}}}{N_{t o t}}
$$


where $N_{\text {det }}$ and $N_{t o t}$ are the detected events and the total number of events (detected and undetected). In this case the uncertainty of the efficiency $\left(\delta \varepsilon_{\text {det }}\right)$ is given by the formula:

$$
\delta \varepsilon_{\mathrm{det}}=\sqrt{\frac{\varepsilon_{\mathrm{det}}\left(1-\varepsilon_{\mathrm{det}}\right)}{N_{\text {tot }}}},
$$

After obtaining the uncertainties for the different efficiency analyses, the standard method for uncertainties propagation is used:

$$
\delta \varepsilon_{\mathrm{det}}=\sqrt{\sum\left(\frac{\partial \varepsilon_{\mathrm{det}}}{\partial x_{i}}\right) \cdot\left(\delta x_{i}\right)^{2}}
$$

where $\frac{\partial \varepsilon_{\text {det }}}{\partial x_{i}}$ is the partial derivative with respect to a generic variable and $\delta x_{i}$ is variation of the generic variable. Because systematic uncertainties result from the uncertainties in the measurements of the detector efficiencies, inefficiencies, and scaling factors, and because they propagate as shown above, they are straightforward to tabulate as shown in Table 46. After obtaining the uncertainties in the correction factors, it is simple to get the systematics uncertainties in the cross sections. The systematics uncertainties are obtained by multiplying the experimental yield by the uncertainties of the correction factors according to Equation (63) instead of the correction factors, and following the same method as when calculating the experimental cross section (see Section 4.8). It is 
important to note that the uncertainties obtained per run in sections $3.3,3.4$ and 3.5 were weighted by the charge by the following equation:

$$
\delta \varepsilon_{\mathrm{det}}=\frac{\sum Q_{i} \delta \varepsilon_{i}}{N \sum Q_{i}}
$$

where $N$ is the number of runs for a particular kinematics/particle selected. Table 46 shows the uncertainties in each correction factor for each detector that contribute for the total uncertainty in the total correction factor.

\begin{tabular}{ccccccccc}
\hline $\begin{array}{c}\text { Kinematics } \\
\text { particle }\end{array}$ & Uncertainty: & \multicolumn{6}{c}{$\begin{array}{c}\text { SYSTEMATIC UNCERTAINTIES IN THE CORRECTION } \\
\text { FACTORS FROM DETECTORS AND ELECTRONICS }\end{array}$} \\
\hline $\begin{array}{c}\text { Uncertainty } \\
\text { in: }\end{array}$ & $\begin{array}{c}\text { Correction } \\
\text { FACTOR }\end{array}$ & VDC & $\mathrm{A} 1$ & $\mathrm{~A} 2$ & $\mathrm{CO}_{2}$ & Deadtime & Scint_eff & Absorp. \\
L_KIN_1 & 0.1110 & 0.0008 & 0.0145 & 0.0021 & 0.0007 & 0.0002 & 0.00003 & 0.0016 \\
L_KIN_2 & 0.1738 & 0.0010 & 0.0210 & 0.0037 & 0.0007 & 0.0001 & 0.00003 & 0.0016 \\
L_KIN_3 & 0.1465 & 0.0009 & 0.0195 & 0.0028 & 0.0012 & 0.0001 & 0.00003 & 0.0015 \\
S_KIN_1 & 0.2814 & 0.0008 & 0.0221 & 0.0090 & 0.0007 & 0.0002 & 0.00003 & 0.0016 \\
S_KIN_2 & 0.2632 & 0.0010 & 0.0356 & 0.0056 & 0.0007 & 0.0001 & 0.00003 & 0.0016 \\
\hline
\end{tabular}

Table 46: Systematic uncertainties from detectors and electronics in the correction factors.

Regarding Table 46 and proceeding as the explanation in the above paragraphs the contribution from detectors and electronics for the systematic uncertainties in the cross-section measurements were calculated and are shown in Table 47.á 


\begin{tabular}{cc}
\hline kinematics/particle & Systematic Uncertainties $(\mu \mathrm{B} / \mathrm{sr})$ \\
\hline L_KIN_1 & 0.0078 \\
L_KIN_2 & 0.0103 \\
L_KIN_3 & 0.0112 \\
S_KIN_1 & 0.0058 \\
S_KIN 2 & 0.0086 \\
\hline
\end{tabular}

Table 47: Systematic uncertainties from detectors and electronics in the experimental cross sections.

In addition to the systematic uncertainties shown in Table 47, the integrated beam current uncertainty and the target density uncertainty that reflect the uncertainty in the luminosity has to be included in the overall systematic uncertainties. The integrated beam current uncertainties are calculated from Table 40 . The target density uncertainties are taken as the estimates described in Section 3.7. These additional uncertainties are shown in Table 48.

\begin{tabular}{ccc}
\hline Kinematics & Target density & Integrated beam current \\
\hline & Uncertainty $(\%)$ & Uncertainty $(\%)$ \\
KIN_1 $(2485-2492)$ & 1.00 & 0.18 \\
KIN_2 $(2496-2508)$ & 1.00 & 0.28 \\
KIN_3 (2509-2521) & 1.00 & 0.24 \\
\hline
\end{tabular}

Table 48: Uncertainties in target density and integrated beam current.

The statistical uncertainties comes from the experimental yields themselves and they are different for each bin done later in Section 4.10. In the case of the five different kinematics/particle selected, the uncertainty in the yield is given by the square root of the uncorrected experimental yield (Table 39), which then correcting the uncertainties and following the same procedure to calculate the cross section as done using the Equation (56), this produces the following results shown in Tables 49 and 50. 


\begin{tabular}{cc}
\hline kinematics/particle & $\begin{array}{c}\text { Statistical Uncertainties } \\
(\mu \mathrm{B} / \mathrm{sr})\end{array}$ \\
\hline L_KIN_1 & 0.0156 \\
L_KIN_2 & 0.0223 \\
L_KIN_3 & 0.0126 \\
S_KIN_1 & 0.0113 \\
S_KIN_2 & 0.0093 \\
\hline
\end{tabular}

Table 49: Statistical uncertainties in the experimental cross sections.

\begin{tabular}{lcccccc}
\hline $\begin{array}{c}\text { Kinematics } \\
\text { /particle }\end{array}$ & $\begin{array}{c}\text { SYSTEMATICS } \\
(\mu \mathrm{B} / \mathrm{sr})\end{array}$ & $\begin{array}{c}\text { STATISTICAL } \\
(\mu \mathrm{B} / \mathrm{sr})\end{array}$ & $\begin{array}{c}\text { TOTAL } \\
(\mu \mathrm{B} / \mathrm{sr})\end{array}$ & $\begin{array}{c}\text { Syst. } \\
\text { Unc. } \\
(\%)\end{array}$ & $\begin{array}{c}\text { Stat. } \\
\text { Unc. } \\
(\%)\end{array}$ & $\begin{array}{c}\text { Total } \\
\%\end{array}$ \\
\hline L_KIN_1 & 0.0104 & 0.0156 & 0.0187 & 1.79 & 2.69 & 3.24 \\
L_KIN_2 & 0.0123 & 0.0223 & 0.0255 & 2.33 & 4.21 & 4.82 \\
L_KIN_3 & 0.0137 & 0.0126 & 0.0186 & 2.16 & 2.00 & 2.94 \\
S_KIN_1 & 0.0065 & 0.0113 & 0.0130 & 2.63 & 4.56 & 5.28 \\
S_KIN_2 & 0.0093 & 0.0093 & 0.0131 & 3.41 & 3.40 & 4.82 \\
\hline
\end{tabular}

Table 50: Summary of uncertainties in E94-107 (Tables 47, 48 and 49 included).

In addition, having a large number of entries (hundreds of thousands) in the MCEEP simulation makes the statistical uncertainties in the simulation results too small to be considered.

\subsection{Data binning}

The purpose of binning the data over the narrow range of kinematics covered in this experiment is to observe the trend or slope of the cross sections when a particular variable changes. In particular the interesting variables are: momentum transfer squared $\left(Q^{2}\right)$, invariant mass $(W)$ and kaon angle in the $\mathrm{CM}$ frame $\left(\theta_{C M}\right)$. As a result of the limited statistics in experiment E94-107, it was decided to bin the data in three approximately equal parts for each variable, producing three cross-section measurements 
for each kinematics/particle selection for each of the five kinematics/particle (Table 21) selections. Given three different variables, a total of forty five cross-section measurements were obtained resulting from the binning. The ranges for the variables taken for the binning, mentioned in the previous paragraph are shown in Tables 51, 52 and 53.

\begin{tabular}{cccc}
\hline $\begin{array}{c}\text { Kinematics } \\
\text { /particle }\end{array}$ & $\mathrm{Q} 1(\mathrm{GeV} / \mathrm{c})^{2}$ & $\mathrm{Q} 2(\mathrm{GeV} / \mathrm{c})^{2}$ & $\mathrm{Q} 3(\mathrm{GeV} / \mathrm{c})^{2}$ \\
\hline L_KIN_1 & {$[0.0400,0.0593]$} & {$[0.0593,0.0756]$} & {$[0.0756,0.1200]$} \\
L_KIN_2 & {$[0.0400,0.0666]$} & {$[0.0666,0.0789]$} & {$[0.0789,0.1200]$} \\
L_KIN_3 & {$[0.0400,0.0613]$} & {$[0.0613,0.0762]$} & {$[0.0762,0.1200]$} \\
\hline S_KIN_1 & {$[0.0400,0.0568]$} & {$[0.0568,0.0677]$} & {$[0.0677,0.1200]$} \\
S_KIN_2 & {$[0.0400,0.0599]$} & {$[0.0599,0.0700]$} & {$[0.0700,0.1200]$} \\
\hline
\end{tabular}

Table 51: Ranges for binning the data in $Q^{2}$ for each kinematics/particle selection. The bins in $Q^{2}$ were labeled; Q1, Q2 and Q3.

\begin{tabular}{cccc}
\hline $\begin{array}{c}\text { Kinematics } \\
\text { /particle }\end{array}$ & W1 $(\mathrm{GeV})$ & W2 $(\mathrm{GeV})$ & W3 $(\mathrm{GeV})$ \\
\hline L_KIN_1 & {$[2.1794,2.2112]$} & {$[2.2112,2.2218]$} & {$[2.2218,2.2694]$} \\
L_KIN_2 & {$[2.1794,2.2036]$} & {$[2.2036,2.2091]$} & {$[2.2091,2.2694]$} \\
L_KIN_3 & {$[2.1794,2.2268]$} & {$[2.2268,2.2382]$} & {$[2.2382,2.2694]$} \\
\hline S_KIN_1 & {$[2.1863,2.2303]$} & {$[2.2303,2.2369]$} & {$[2.2369,2.2583]$} \\
S_KIN_2 & {$[2.1863,2.2205]$} & {$[2.2205,2.2359]$} & {$[2.2359,2,2583]$} \\
\hline
\end{tabular}

Table 52: Ranges for binning the data in $W$ for each kinematics/particle selection. The bins in $W$ were labeled; W1, W2 and W3.

\begin{tabular}{cccc}
\hline $\begin{array}{c}\text { Kinematics } \\
\text { particle }\end{array}$ & T1 (rad) & T2 $(\mathrm{rad})$ & T3 $(\mathrm{rad})$ \\
\hline L_KIN_1 & {$[0.0000,0.0923]$} & {$[0.0923,0.1283]$} & {$[0.1283,0.2550]$} \\
L_KIN_2 & {$[0.0000,0.0892]$} & {$[0.0892,0.1194]$} & {$[0.1194,0.2550]$} \\
L_KIN_3 & {$[0.0000,0.0942]$} & {$[0.0942,0.1312]$} & {$[0.1312,0.2550]$} \\
\hline S_KIN_1 & {$[0.0000,0.0860]$} & {$[0.0860,0.1206]$} & {$[0.1206,0.2550]$} \\
S_KIN_2 & {$[0.0000,0.0916]$} & {$[0.0916,0.1280]$} & {$[0.1280,0.2550]$} \\
\hline
\end{tabular}

Table 53: Ranges for binning the data in $\theta_{C M}$ for each kinematics/particle selection. The bins in $\theta_{C M}$ were labeled; T1, T2 and T3. 
Another piece of information needed is the input average values from the MCEEP simulation to be used as input to the program KFITs3.f that calculates the "point" cross section for each bin (see Section 4.8). The point cross sections are multiplied by the ratio of the corrected measured yield to the MCEEP simulated yield (see Equations from (56) to (60)). MCEEP values were used as input to KFIT3s.f instead of real data because the statistical uncertainties in the simulation (high number of entries) were neglectable compared to the significant statistical experimental uncertainties (see Table 50), and a good matching between the simulated and experimental yields was obtained after acceptance corrections (see Section 4.7). Detailed information of the MCEEP summary outputs and the comparison of the MCEEP histograms and the experimental histograms are in Appendix J.

The MCEEP values are listed for the binned data in the variables $Q^{2}, W$ and $\theta_{C M}$ in Tables 54 to 56 , where variations on the average values of the unbinned variables are observed, because when binning in one variable no cuts were applied in the other variables to keep statistics as high as possible, otherwise the statistical uncertainties increases too much. 


\begin{tabular}{cccccc}
\hline $\begin{array}{c}\text { Binned } \\
\text { Kinematics } \\
\text { /particle }\end{array}$ & $\begin{array}{c}\text { Beam } \\
\text { Energy }(E) \\
(\mathrm{GeV})\end{array}$ & $\begin{array}{c}Q^{2} \\
(\mathrm{GeV} / \mathrm{c})^{2}\end{array}$ & $\begin{array}{c}W \\
(\mathrm{GeV})\end{array}$ & $\begin{array}{c}p_{K} \\
(\mathrm{GeV} / \mathrm{c})\end{array}$ & $\begin{array}{c}\theta_{K} \\
(\text { degrees })\end{array}$ \\
\hline Q1_L_KIN_1 & 3.7758 & 0.0540 & 2.2200 & 1.8961 & 2.6400 \\
Q2_L_KIN_1 & 3.7758 & 0.0670 & 2.2170 & 1.8944 & 2.4010 \\
Q3_L_KIN_1 & 3.7758 & 0.0880 & 2.2110 & 1.8917 & 1.9800 \\
\hline Q1_L_KIN_2 & 3.7807 & 0.0590 & 2.2100 & 1.8724 & 2.5580 \\
Q2_L_KIN_2 & 3.7807 & 0.0730 & 2.2070 & 1.8723 & 2.3100 \\
Q3_L_KIN_2 & 3.7807 & 0.0910 & 2.2030 & 1.8718 & 1.9490 \\
\hline Q1_L_KIN_3 & 3.7758 & 0.0550 & 2.2350 & 1.9348 & 2.7110 \\
Q2_L_KIN_3 & 3.7758 & 0.0690 & 2.2310 & 1.9326 & 2.4420 \\
Q3_L_KIN_3 & 3.7758 & 0.0880 & 2.2250 & 1.9298 & 2.0270 \\
\hline Q1_S_KIN_1 & 3.7758 & 0.0530 & 2.2370 & 1.8317 & 2.7540 \\
Q2_S_KIN_1 & 3.7758 & 0.0620 & 2.2340 & 1.8302 & 2.5790 \\
Q3_S_KIN_1 & 3.7758 & 0.0830 & 2.2290 & 1.8284 & 2.1330 \\
\hline Q1_S_KIN_2 & 3.7807 & 0.0550 & 2.2310 & 1.8174 & 2.6990 \\
Q2_S_KIN_2 & 3.7807 & 0.0650 & 2.2270 & 1.8139 & 2.5150 \\
Q3_S_KIN_2 & 3.7807 & 0.0850 & 2.2220 & 1.8099 & 2.0950 \\
\hline
\end{tabular}

Table 54: MCEEP average values for data binning in $Q^{2}$. The bins in $Q^{2}$ were labeled; Q1, Q2 and Q3.

\begin{tabular}{cccccc}
\hline $\begin{array}{c}\text { Binned } \\
\text { Kinematics } \\
\text { /particle }\end{array}$ & $\begin{array}{c}\text { Beam } \\
\text { Energy }(E) \\
(\mathrm{GeV})\end{array}$ & $\begin{array}{c}Q^{2} \\
(\mathrm{GeV} / \mathrm{c})^{2}\end{array}$ & $\begin{array}{c}W \\
(\mathrm{GeV})\end{array}$ & $\begin{array}{c}p_{K} \\
(\mathrm{GeV} / \mathrm{c})\end{array}$ & $\begin{array}{c}\theta_{K} \\
(\text { degrees })\end{array}$ \\
\hline W1_L_KIN_1 & 3.7758 & 0.0790 & 2.2030 & 1.8682 & 2.0910 \\
W2_L_KIN_1 & 3.7758 & 0.0750 & 2.2160 & 1.8997 & 2.2410 \\
W3_L_KIN_1 & 3.7758 & 0.0700 & 2.2290 & 1.9232 & 2.4550 \\
\hline W1_L_KIN_2 & 3.7807 & 0.0840 & 2.1990 & 1.8625 & 2.0060 \\
W2_L_KIN_2 & 3.7807 & 0.0750 & 2.2060 & 1.8746 & 2.2330 \\
W3_L_KIN_2 & 3.7807 & 0.0690 & 2.2150 & 1.8815 & 2.4750 \\
\hline W1_L_KIN_3 & 3.7758 & 0.0780 & 2.2170 & 1.9064 & 2.1600 \\
W2_L_KIN_3 & 3.7758 & 0.0730 & 2.2320 & 1.9382 & 2.3760 \\
W3_L_KIN_3 & 3.7758 & 0.0680 & 2.2440 & 1.9645 & 2.5420 \\
\hline W1_S_KIN_1 & 3.7758 & 0.0780 & 2.2230 & 1.8122 & 2.1520 \\
W2_S_KIN_1 & 3.7758 & 0.0730 & 2.2340 & 1.8337 & 2.3510 \\
W3_S_KIN_1 & 3.7758 & 0.0670 & 2.2420 & 1.8496 & 2.5700 \\
\hline W1_S_KIN_2 & 3.7807 & 0.0780 & 2.2090 & 1.7782 & 2.1330 \\
W2_S_KIN_2 & 3.7807 & 0.0740 & 2.2280 & 1.8194 & 2.3420 \\
W3_S_KIN_2 & 3.7807 & 0.0700 & 2.2430 & 1.8544 & 2.5130 \\
\hline
\end{tabular}

Table 55: MCEEP average values for data binning in $W$. The bins in $W$ were labeled; $\mathrm{W} 1, \mathrm{~W} 2$ and $\mathrm{W} 3$. 


\begin{tabular}{cccccc}
\hline $\begin{array}{c}\text { Binned } \\
\text { Kinematics } \\
\text { /particle }\end{array}$ & $\begin{array}{c}\text { Beam } \\
\text { Energy }(E)\end{array}$ & $\begin{array}{c}Q^{2} \\
(\mathrm{GeV} / \mathrm{c})^{2}\end{array}$ & $\begin{array}{c}W \\
(\mathrm{GeV})\end{array}$ & $\begin{array}{c}p_{K} \\
(\mathrm{GeV} / \mathrm{c})\end{array}$ & $\begin{array}{c}\theta_{K} \\
(\text { degrees })\end{array}$ \\
\hline T1_L_KIN_1 & 3.7758 & 0.0800 & 2.2120 & 1.8922 & 1.4010 \\
T2_L_KIN_1 & 3.7758 & 0.0730 & 2.2150 & 1.8944 & 2.4110 \\
T3_L_KIN_1 & 3.7758 & 0.0700 & 2.2180 & 1.8940 & 3.3650 \\
\hline T1_L_KIN_2 & 3.7807 & 0.0820 & 2.2040 & 1.8728 & 1.3630 \\
T2_L_KIN_2 & 3.7807 & 0.0750 & 2.2070 & 1.8725 & 2.2880 \\
T3_L_KIN_2 & 3.7807 & 0.0720 & 2.2080 & 1.8709 & 3.2340 \\
\hline T1_L_KIN_3 & 3.7758 & 0.0790 & 2.2260 & 1.9299 & 1.4850 \\
T2_L_KIN_3 & 3.7758 & 0.0720 & 2.2300 & 1.9330 & 2.4540 \\
T3_L_KIN_3 & 3.7758 & 0.0680 & 2.2330 & 1.9335 & 3.3920 \\
\hline T1_S_KIN_1 & 3.7758 & 0.0800 & 2.2290 & 1.8278 & 1.3410 \\
T2_S_KIN_1 & 3.7758 & 0.0730 & 2.2310 & 1.8294 & 2.2400 \\
T3_S_KIN_1 & 3.7758 & 0.0680 & 2.2340 & 1.8304 & 3.2110 \\
\hline T1_S_KIN_2 & 3.7807 & 0.0800 & 2.2210 & 1.8083 & 1.4280 \\
T2_S_KIN_2 & 3.7807 & 0.0730 & 2.2260 & 1.8139 & 2.3730 \\
T3_S_KIN_2 & 3.7807 & 0.0690 & 2.2280 & 1.8152 & 3.3210 \\
\hline
\end{tabular}

Table 56: MCEEP average values for data binning in $\theta_{C M}$. The bins in $\theta_{C M}$ were labeled; $\mathrm{T} 1, \mathrm{~T} 2$ and $\mathrm{T} 3$.

The method followed to obtain the cross sections was the same as explained in Section 4.8. The results have been listed in Tables 57, 58 and 59. The first column indicates the different bins, the second the yield in each bin, the third column indicates the average value of the variable on which the binning was performed, then follows the measured cross section, and the four final columns are the uncertainties, which were obtained by the procedure explained in Section 4.9. The results are shown graphically in Chapter 5, Section 5.1. 


\begin{tabular}{cccccccc}
\hline $\begin{array}{c}\text { Binned } \\
\text { Kinematics } \\
\text { /particle }\end{array}$ & $\begin{array}{c}\text { Binned } \\
\text { Yield }\end{array}$ & $\begin{array}{c}\text { Average } \\
Q^{2} \\
(\mathrm{GeV} / \mathrm{c})^{2}\end{array}$ & $\begin{array}{c}\text { Cross } \\
\text { Section } \\
(\mu \mathrm{B} / \mathrm{sr})\end{array}$ & $\begin{array}{c}\text { Systematic } \\
\text { Uncert. } \\
(\mu \mathrm{B} / \mathrm{sr})\end{array}$ & $\begin{array}{c}\text { Statistical } \\
\text { Uncert. } \\
(\mu \mathrm{B} / \mathrm{sr})\end{array}$ & $\begin{array}{c}\text { Total } \\
\text { Uncert. } \\
(\mu \mathrm{B} / \mathrm{sr})\end{array}$ & $\begin{array}{c}\text { Total } \\
\text { Uncer. } \\
(\%)\end{array}$ \\
\hline Q1_L_KIN_1 & 399 & 0.0540 & 0.7280 & 0.0104 & 0.0364 & 0.0379 & 5.20 \\
Q2_L_KIN_1 & 450 & 0.0670 & 0.5004 & 0.0104 & 0.0236 & 0.0258 & 5.15 \\
Q3_L_KIN_1 & 531 & 0.0880 & 0.5672 & 0.0104 & 0.0246 & 0.0267 & 4.71 \\
\hline Q1_L_KIN_2 & 245 & 0.0590 & 0.6210 & 0.0123 & 0.0397 & 0.0416 & 6.69 \\
Q2_L_KIN_2 & 135 & 0.0730 & 0.4806 & 0.0123 & 0.0414 & 0.0432 & 8.99 \\
Q3_L_KIN_2 & 181 & 0.0910 & 0.4683 & 0.0123 & 0.0348 & 0.0369 & 7.88 \\
\hline Q1_L_KIN_3 & 881 & 0.0550 & 0.7177 & 0.0137 & 0.0242 & 0.0278 & 3.87 \\
Q2_L_KIN_3 & 788 & 0.0690 & 0.5774 & 0.0137 & 0.0206 & 0.0247 & 4.28 \\
Q3_L_KIN_3 & 837 & 0.0880 & 0.6110 & 0.0137 & 0.0211 & 0.0252 & 4.12 \\
\hline Q1_S_KIN_1 & 128 & 0.0530 & 0.3313 & 0.0065 & 0.0293 & 0.0300 & 9.06 \\
Q2_S_KIN_1 & 149 & 0.0620 & 0.2775 & 0.0065 & 0.0227 & 0.0236 & 8.51 \\
Q3_S_KIN_1 & 203 & 0.0830 & 0.1995 & 0.0065 & 0.0140 & 0.0154 & 7.74 \\
\hline Q1_S_KIN_2 & 225 & 0.0550 & 0.2883 & 0.0093 & 0.0192 & 0.0213 & 7.40 \\
Q2_S_KIN_2 & 215 & 0.0650 & 0.2694 & 0.0093 & 0.0184 & 0.0206 & 7.65 \\
Q3_S_KIN_2 & 425 & 0.0850 & 0.2670 & 0.0093 & 0.0130 & 0.0160 & 5.99 \\
\hline
\end{tabular}

Table 57: Cross-section results for data binning in $Q^{2}$, summed over the other variables. The bins in $Q^{2}$ were labeled; Q1, Q2 and Q3.

\begin{tabular}{cccccccc}
\hline $\begin{array}{c}\text { Binned } \\
\text { Kinematics } \\
\text { /particle }\end{array}$ & $\begin{array}{c}\text { Binned } \\
\text { Yield }\end{array}$ & $\begin{array}{c}\text { Average } \\
W \\
(\mathrm{GeV})\end{array}$ & $\begin{array}{c}\text { Cross } \\
\text { Section } \\
(\mu \mathrm{B} / \mathrm{sr})\end{array}$ & $\begin{array}{c}\text { Systematical } \\
\text { Uncert. } \\
(\mu \mathrm{B} / \mathrm{sr})\end{array}$ & $\begin{array}{c}\text { Statistical } \\
\text { Uncert. } \\
(\mu \mathrm{B} / \mathrm{sr})\end{array}$ & $\begin{array}{c}\text { Total } \\
\text { Uncert. } \\
(\mu \mathrm{B} / \mathrm{sr})\end{array}$ & $\begin{array}{c}\text { Total } \\
(\%)\end{array}$ \\
\hline W1_L_KIN_1 & 513 & 2.2030 & 0.5274 & 0.0104 & 0.0233 & 0.0255 & 4.84 \\
W2_L_KIN_1 & 382 & 2.2160 & 0.5699 & 0.0104 & 0.0292 & 0.0310 & 5.44 \\
W3_L_KIN_1 & 484 & 2.2290 & 0.6503 & 0.0104 & 0.0296 & 0.0314 & 4.82 \\
\hline W1_L_KIN_2 & 179 & 2.1990 & 0.4534 & 0.0123 & 0.0339 & 0.0361 & 7.95 \\
W2_L_KIN_2 & 192 & 2.2060 & 0.5930 & 0.0123 & 0.0428 & 0.0445 & 7.51 \\
W3_L_KIN_2 & 191 & 2.2150 & 0.5539 & 0.0123 & 0.0401 & 0.0419 & 7.57 \\
\hline W1_L_KIN_3 & 958 & 2.2170 & 0.5865 & 0.0137 & 0.0189 & 0.0233 & 3.98 \\
W2_L_KIN_3 & 737 & 2.2320 & 0.6555 & 0.0137 & 0.0241 & 0.0277 & 4.23 \\
W3_L_KIN_3 & 811 & 2.2440 & 0.6721 & 0.0137 & 0.0236 & 0.0273 & 4.06 \\
\hline W1_S_KIN_1 & 212 & 2.2230 & 0.2520 & 0.0065 & 0.0173 & 0.0185 & 7.33 \\
W2_S_KIN_1 & 129 & 2.2340 & 0.2947 & 0.0065 & 0.0259 & 0.0267 & 9.06 \\
W3_S_KIN_1 & 138 & 2.2420 & 0.2073 & 0.0065 & 0.0176 & 0.0188 & 9.05 \\
\hline W1_S_KIN_2 & 348 & 2.2090 & 0.2819 & 0.0093 & 0.0151 & 0.0177 & 6.29 \\
W2_S_KIN_2 & 259 & 2.2280 & 0.2626 & 0.0093 & 0.0163 & 0.0188 & 7.15 \\
W3_S_KIN_2 & 258 & 2.2430 & 0.2712 & 0.0093 & 0.0169 & 0.0193 & 7.11 \\
\hline
\end{tabular}

Table 58: Cross-section results for data binning in $W$, summed over the other variables. The bins in $W$ were labeled; W1, W2 and W3. 


\begin{tabular}{cccccccc}
\hline $\begin{array}{c}\text { Binned } \\
\text { Kinematics } \\
\text { /particle }\end{array}$ & $\begin{array}{c}\text { Binned } \\
\text { Yield }\end{array}$ & $\begin{array}{c}\text { Average } \\
\theta_{\mathrm{CM}} \\
(\mathrm{deg} .)\end{array}$ & $\begin{array}{c}\text { Cross } \\
\text { Section } \\
(\mu \mathrm{B} / \mathrm{sr})\end{array}$ & $\begin{array}{c}\text { Systematical } \\
\text { Uncert. } \\
(\mu \mathrm{B} / \mathrm{sr})\end{array}$ & $\begin{array}{c}\text { Statistical } \\
\text { Uncert. } \\
(\mu \mathrm{B} / \mathrm{sr})\end{array}$ & $\begin{array}{c}\text { Total } \\
\text { Uncert. } \\
(\mu \mathrm{B} / \mathrm{sr})\end{array}$ & $\begin{array}{c}\text { Total } \\
\text { Uncert. } \\
(\%)\end{array}$ \\
\hline T1_L_KIN_1 & 484 & 3.6600 & 0.5191 & 0.0104 & 0.0236 & 0.0258 & 4.97 \\
T2_L_KIN_1 & 451 & 6.2900 & 0.5767 & 0.0104 & 0.0272 & 0.0291 & 5.05 \\
T3_L_KIN_1 & 444 & 8.7730 & 0.6602 & 0.0104 & 0.0313 & 0.0330 & 5.00 \\
\hline T1_L_KIN_2 & 186 & 3.5530 & 0.4665 & 0.0123 & 0.0342 & 0.0363 & 7.79 \\
T2_L_KIN_2 & 142 & 5.9590 & 0.4700 & 0.0123 & 0.0394 & 0.0413 & 8.78 \\
T3_L_KIN_2 & 235 & 8.4170 & 0.6481 & 0.0123 & 0.0423 & 0.0441 & 6.80 \\
\hline T1_L_KIN_3 & 840 & 3.8920 & 0.5885 & 0.0137 & 0.0203 & 0.0245 & 4.16 \\
T2_L_KIN_3 & 784 & 6.4290 & 0.5599 & 0.0137 & 0.0200 & 0.0242 & 4.33 \\
T3_L_KIN_3 & 881 & 8.8790 & 0.7745 & 0.0137 & 0.0261 & 0.0295 & 3.81 \\
\hline T1_S_KIN_1 & 145 & 3.5550 & 0.2705 & 0.0065 & 0.0225 & 0.0234 & 8.66 \\
T2_S_KIN_1 & 165 & 5.9310 & 0.2625 & 0.0065 & 0.0204 & 0.0214 & 8.16 \\
T3_S_KIN_1 & 170 & 8.4930 & 0.2184 & 0.0065 & 0.0167 & 0.0179 & 8.21 \\
\hline T1_S_KIN_2 & 297 & 3.7780 & 0.2756 & 0.0093 & 0.0160 & 0.0185 & 6.71 \\
T2_S_KIN_2 & 227 & 6.2740 & 0.2051 & 0.0093 & 0.0136 & 0.0165 & 8.03 \\
T3_S_KIN_2 & 341 & 8.7760 & 0.3452 & 0.0093 & 0.0187 & 0.0209 & 6.05 \\
\hline
\end{tabular}

Table 59: Cross-section results for data binning in $\theta_{C M}$ summed over the other variables. The bins in $\theta_{C M}$ were labeled; T1, T2 and T3.

$5 \quad$ Results and conclusions

In the beginning of this dissertation, Section 1.5 posed the following Question a): What are the cross sections for ${ }^{1} \mathrm{H}\left(e, e^{\prime} K^{+}\right) \Lambda, \Sigma^{0}$ at $\theta_{C M}=6^{\circ}, W=2.2 \mathrm{GeV}$ and $Q^{2} \sim 0.07$ $(\mathrm{GeV} / \mathrm{c})^{2}$ ? The answer is in Section 5.1, where the results from E94-107 are summarized as well as shown graphically.

In Section 5.2, discussions of the results and conclusions are presented to answer Questions b), c), d) and e).

\section{$5.1 \quad$ Results}

The results for the $\Lambda$ channel (Table 45) corresponding to the three first rows of Table 42 are plotted vs. their average kaon momentum and shown in Figure 102. 
Similarly the results for the $\Sigma^{0}$ channel corresponding (Table 45) to the last two rows of Table 42 are plotted vs. their average kaon momentum and shown in Figure 103. In both cases, the inner error bars represent the statistical uncertainties, while the outer error bars represent the total uncertainties (statistical and systematical uncertainties). It is important to mention that the average of the other variables like $Q^{2}$ and $W$ are not quite equal but they are close (e.g., typically a few $\mathrm{MeV}$ in $W$ ) because of the very small acceptance in the Hall A spectrometers. Therefore to answer Questiona a), the cross sections for ${ }^{1} \mathrm{H}\left(e, e^{\prime} K^{+}\right) \Lambda, \Sigma^{0}$ at $\theta_{\mathrm{CM}}=6^{\circ}, W=2.2 \mathrm{GeV}$ and $Q^{2} \sim 0.07(\mathrm{GeV} / \mathrm{c})^{2}$ are shown in Figures 102 and 103 for each kinematics/particle selection (Table 21).

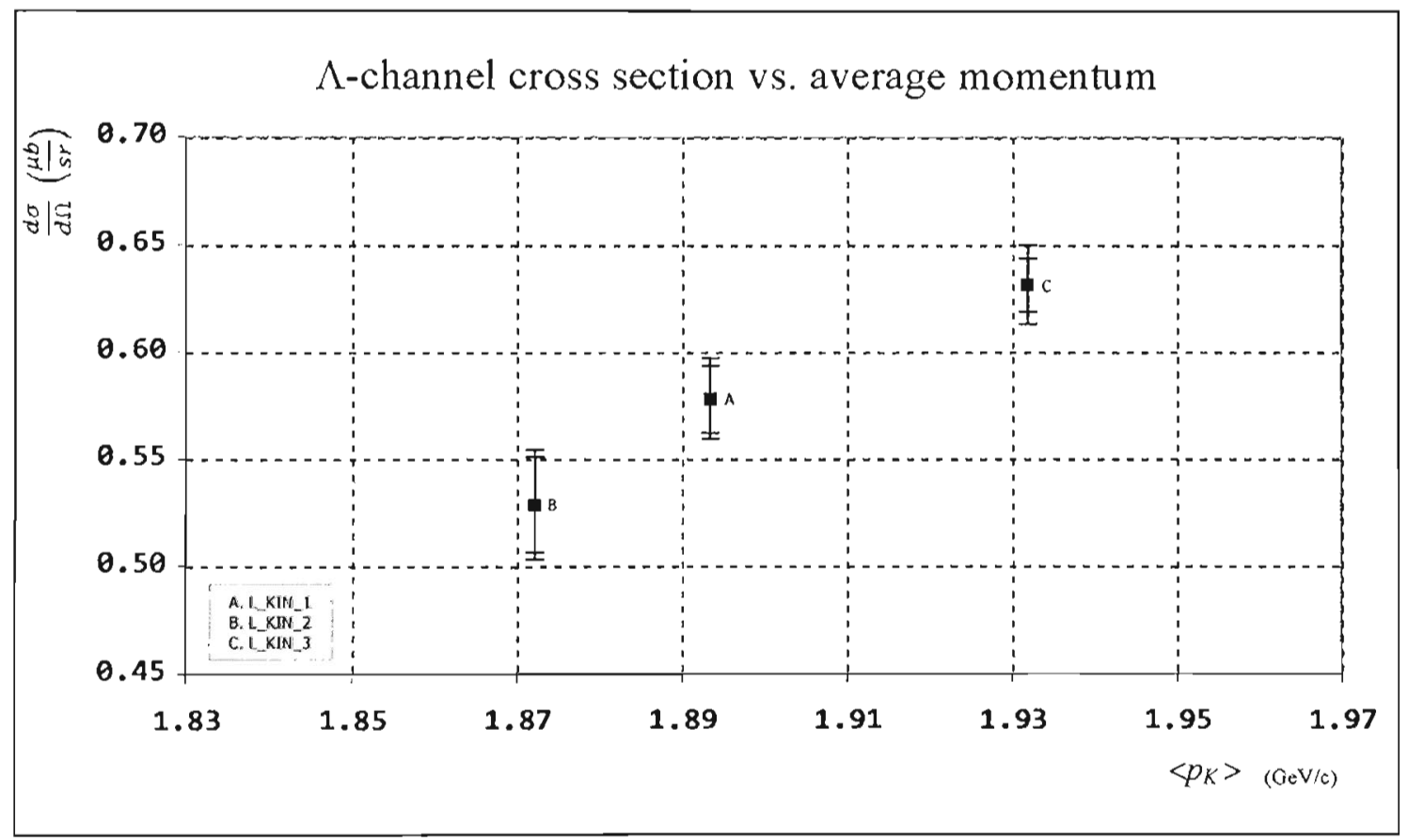

Figure 102: $\Lambda$-channel measured cross section vs. average kaon momentum for unbinned data. $W=2.2 \mathrm{GeV}, Q^{2} \sim 0.07(\mathrm{GeV} / \mathrm{c})^{2}$ and $\theta_{\mathrm{CM}}=6^{\circ}$. Inner error bars represent statisitical uncertainties only, and outer error bars represent total uncertainties (statistical and systematic uncertainties). 


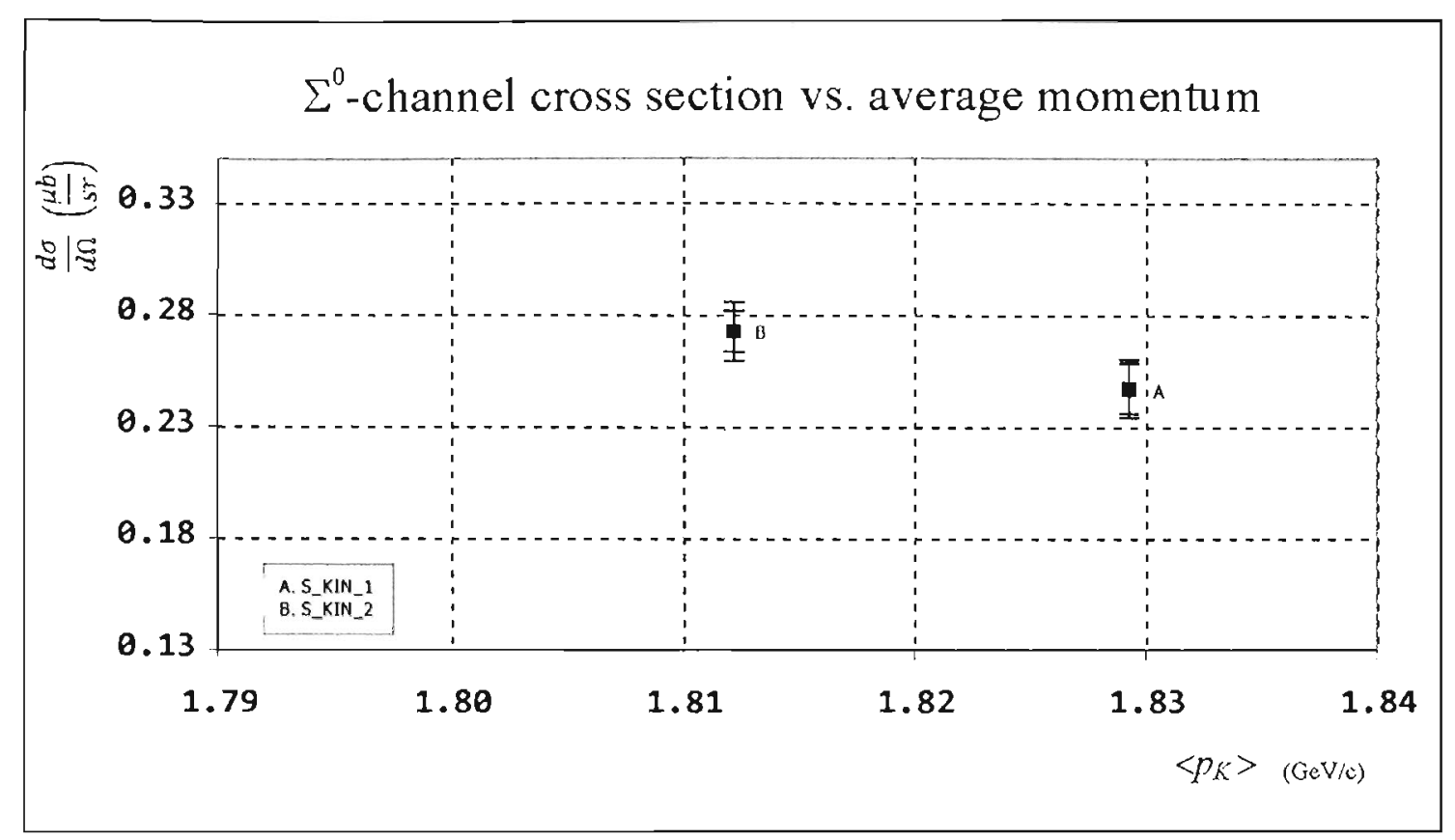

Figure 103: $\Sigma^{0}$ channel measured cross sections vs. average kaon momentum for unbinned data. $W=2.2 \mathrm{GeV}, Q^{2} \sim 0.07(\mathrm{GeV} / \mathrm{c})^{2}$ and $\theta_{\mathrm{CM}}=6^{\circ}$. Inner error bars represent statisitical uncertainties only, and Outer error bars represent total uncertainties (statistical and systematic uncertainties).

The results from the binned data in $Q^{2}, W$ and $\theta_{\mathrm{CM}}$ that are listed in Tables 57, 58 and 59 are shown in Figures 104, 105 and 106. The results are organized for the five different kinematics/particle selections as described in Table 21 . The error bars represent total uncertainties; the combined systematic and statistical uncertainties. 


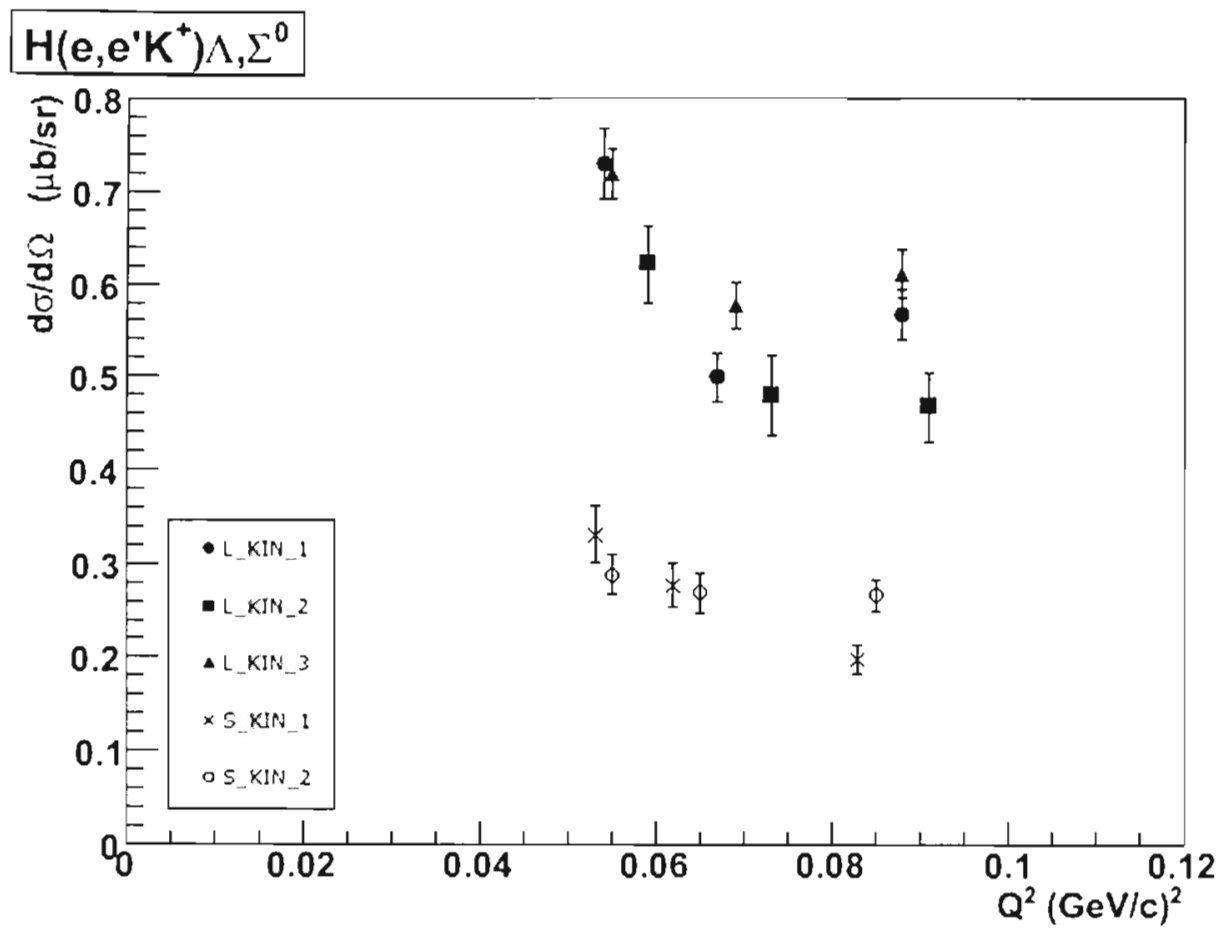

Figure 104: Cross sections vs. $Q^{2}$ in E94-107. Error bars represent total uncertainties (statistical and systematic uncertainties). Average variable values are listed in Table 57.

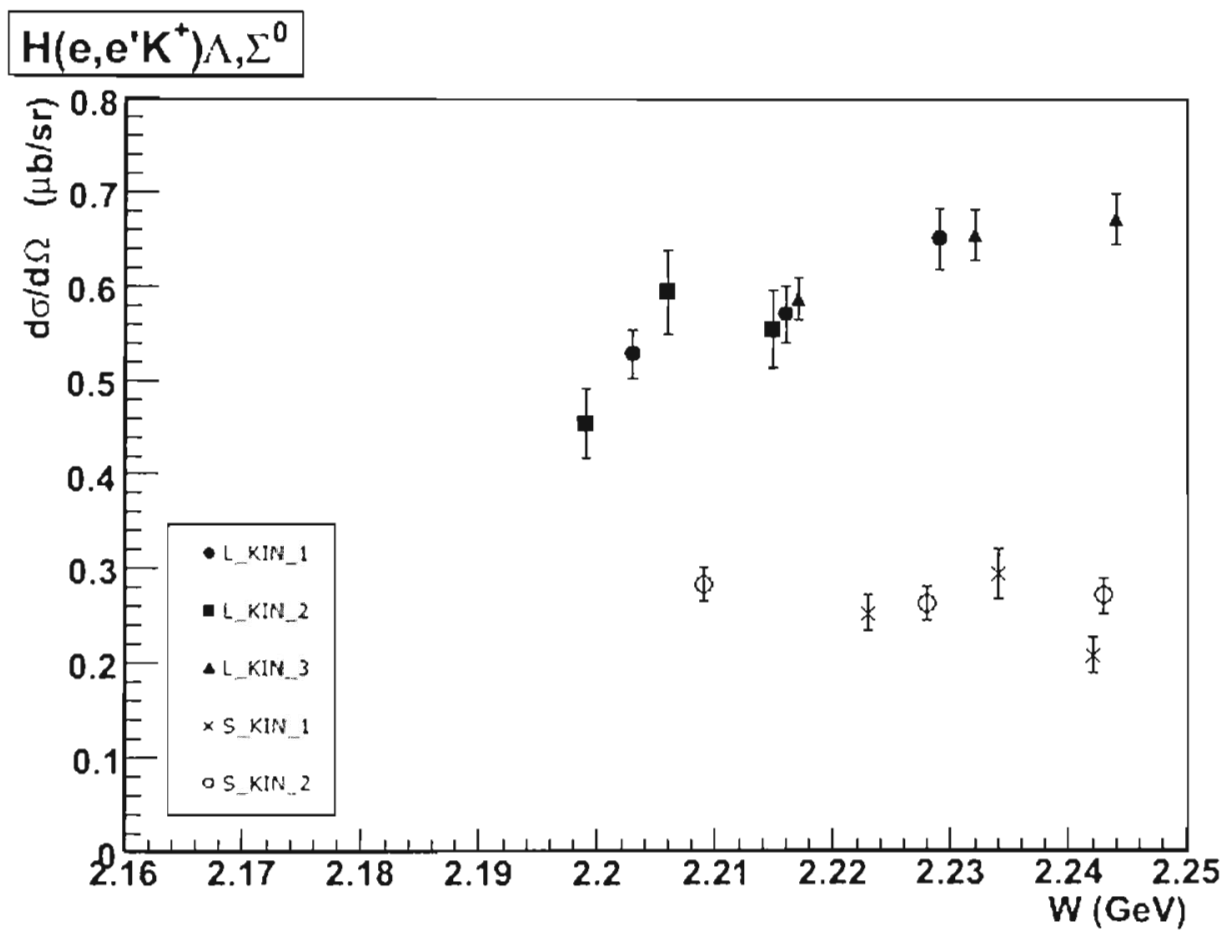

Figure 105: Cross sections vs. $W$ in E94-107. Error bars represent total uncertainties (statistical and systematic uncertainties). Average variable values are listed in Table 58. 


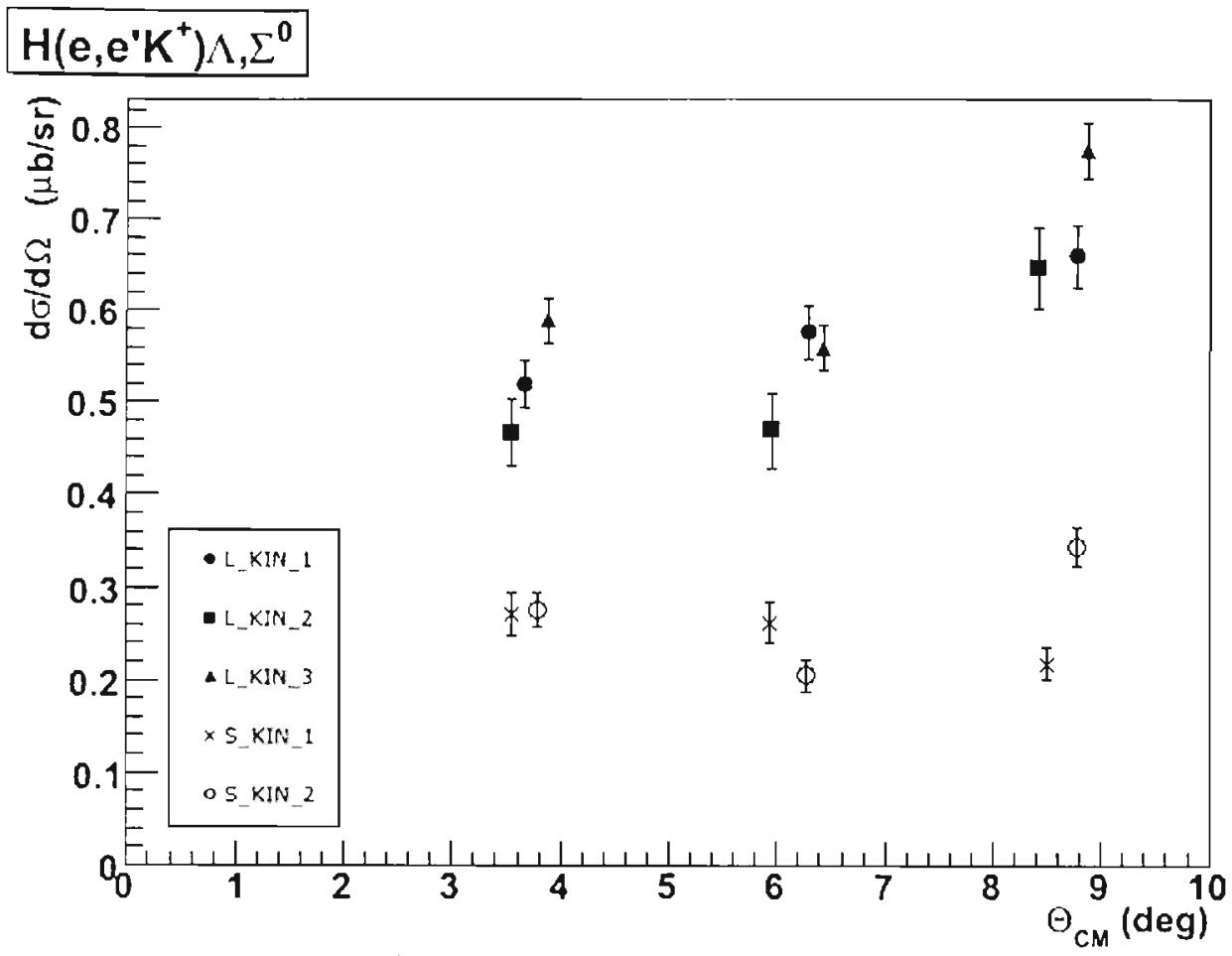

Figure 106: Cross section vs. $\theta_{C M}$ in E94-107. Error bars represent total uncertainties (statistical and systematic uncertainties). Average variable values are listed in Table 59.

Figure 104 shows that the $\Lambda$ channel cross section slightly decreases with $Q^{2}$, but Figure 106 shows that it slightly increases in $\theta_{C M}$. However, according to Figure 105 the trend appears almost flat in $W$. These results are expected according to the scaling factors mentioned in Equations (51) and (54). Note also that the change in $W$ is very small, a possible reason for observing an almost flat trend.

On the other hand, Figures 104, 105 and 106 show that all the trends for the $\Sigma^{0}$ channel are almost flat. Although, it is difficult to conclude from the binning in $Q^{2}$, it can arguably be said that there is a slight decrease when $Q^{2}$ increases. The $\Sigma^{0}$ channel results are less understood than the $\Lambda$ channel. There are not much data available and less 
knowledge about it. One of the scopes of my dissertation is to provide extra data that could be useful for future model construction.

\subsection{Conclusions}

The results of experiment E94-107 are shown in Section 5.1. In this section several comparisons of the results from E94-107 with the existing data and the available models are performed. Furthermore, Questions b), c), d) and e) in Section 1.5 are answered. These questions are:

b) What is the estimated transverse component of the electroproduction cross section? Which model better describes the data with the new experimental point included in Figure 8?

c) Does the cross section for photoproduction continue rising as the kaon angles goes to zero or is there a plateau?

d) What is the ratio of $\Sigma^{0} / \Lambda$ at low $Q^{2}$ ? How does this result compare to the existing data?

e) Which of the models describes the data at forward angles and can therefore be used in the analysis of the hypernuclear data without introducing an additional uncertainty? 
ln order to compare E94-107 results with the existing data and considering that small variations in $\delta p / p$ were done over a fairly small acceptance, the results for the different $\Lambda$ channels (Table 45) and their uncertainties (Table 50) were averaged by the charge for each of the channels (see Equation (60) and (64)). The result obtained is then $0.5858 \pm 0.0208 \frac{\mu \mathrm{b}}{\mathrm{sr}}$ and is shown graphically in Figure 107.

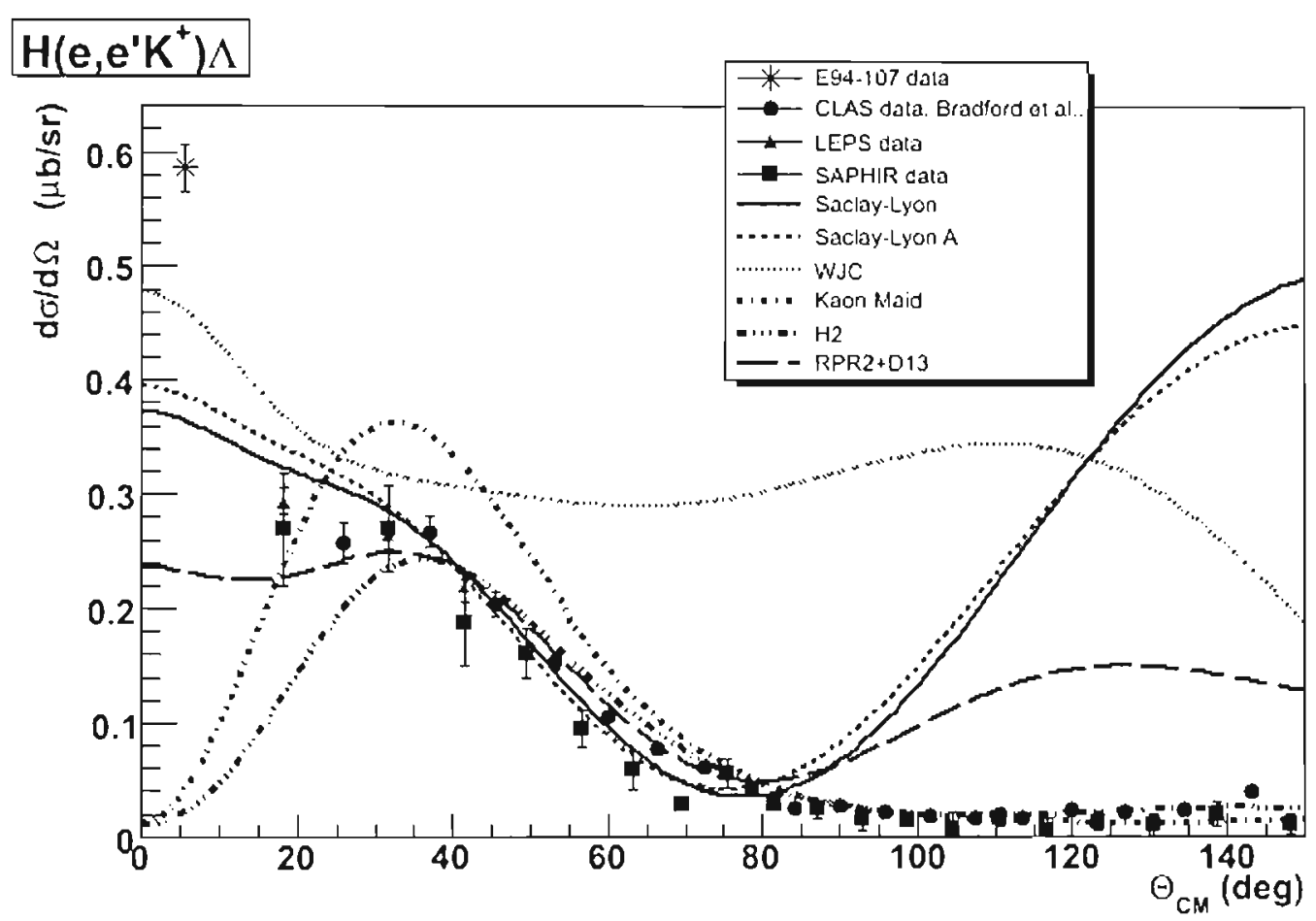

Figure 107: E94-107 electroproduction result with $Q^{2} \sim 0.07(\mathrm{GeV} / \mathrm{c})^{2}, W=2.2 \mathrm{GeV}$ and $\theta_{\mathrm{CM}}=6^{\circ}$ compared to photoproduction data and models, all of them with $W=2.2 \mathrm{GeV}$. Error bars in E94-107 result represent total uncertainty (statistical and systematic uncertainties). CLAS data from Bradford et al., [27].

As shown in Figure 107, the cross section for electroproduction obtained $\theta_{\mathrm{CM}}=6^{\circ}$ from experiment E94-107 is greater than any value of the cross section for 
photoproduction predicted by the models. There is also a lack of experimental data for angles less than $\theta_{\mathrm{CM}}=20^{\circ}$. However, any comparison has to take into account that the cross section for electroproduction in Equation (19), even at low $Q^{2}$, contains extra terms (longitudinal contributions) that make it different from the (strictly transverse) cross section for photoproduction. The cross section for photoproduction does not have either longitudinal or longitudinal-transverse interference terms as is shown in (19) for the electroproduction cross section. In order to proceed with a better comparison, it is required to estimate how significant are the contributions for longitudinal and interference terms in the electroproduction cross section for the E94-107 kinematics. Experiment E94-107 was run not at the same kinematic conditions of the experiment E91-016 [77]. However, we are in a kinematical region in $W$ where there are no resonances dominating and where previous measurements by Bradford et al. [27] have shown that the interference terms are negligible. Therefore, only the longitudinal contribution needs to be estimated. Figure 108 shows the existing data on electroproduction of $\Lambda$ hyperons at JLab. The data include Halls A, B and C (a short description of the experimental halls at JLab is in Section 2.1). Figure 108 shows the different measurements of the cross sections vs. $Q^{2}$ for different invariant masses $W$. For comparison purposes, the data have been scaled in $W$. Consequently, it can be estimated that over the entire range in $Q^{2}$, the ratio $\frac{\sigma_{L}}{\sigma_{T}}$ is not more than 0.5 . In order words, $\frac{\sigma_{L}}{\sigma_{T}}<0.5$ and with Equation (19) it can be stated that: $\frac{d^{2} \sigma}{d \Omega^{*}{ }_{K}} \approx \sigma_{T}+\varepsilon \sigma_{L}$. Observing the E94-107 kinematic settings for the virtual photon's polarization, Figures 91, 92 and 93 
show that the average polarization is $\varepsilon=0.710 \pm 0.010$ for this experiment. Therefore, the estimated contribution by transverse amplitudes to the electroproduction cross section is conservatively greater than $0.740 \pm 0.003$ of the measured cross section. Furthermore, the minimum value that the corresponding photoproduction cross section for E94-107 can reach is given by multiplying $0.740 \pm 0.003$ times the weighted measured electroproduction cross sections and their uncertainties by the charge for each kinematics as shown in Equation (60).

\section{Forward Angle $\Lambda$ L:T Ratio, all W}

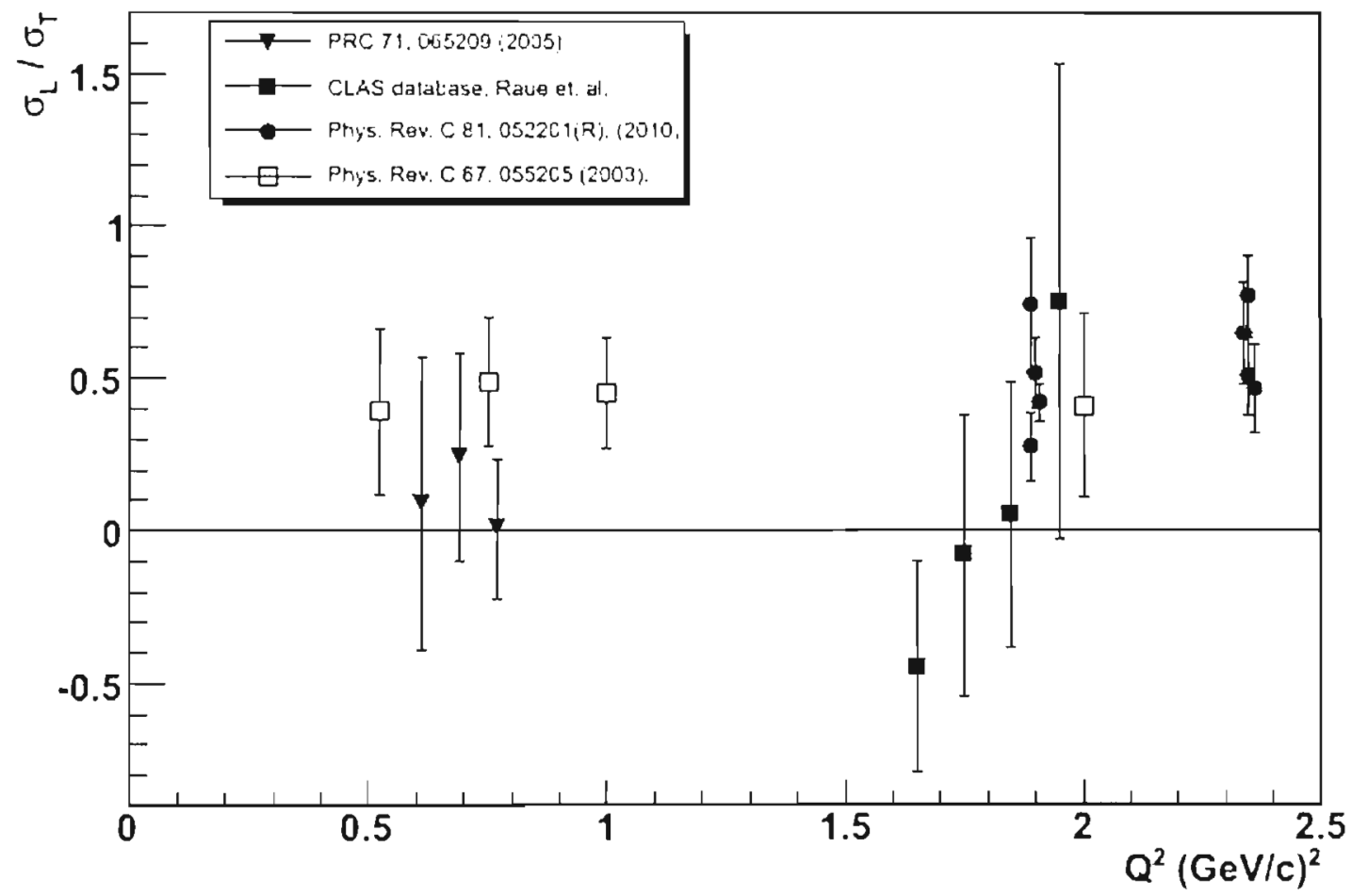

Figure 108: Longitudinal to transversal component ratios of electroproduction cross section for JLab experiments. Data shown are at different $\theta_{\mathrm{CM}}$ and $W$ (on the range 1.5 $1.8 \mathrm{GeV})$. 
Consequently, to answer Questions b) and c), it can be stated that the transverse component of the electroproduction cross section in E94-107 would have a minimum possible value of $0.432 \pm 0.015 \frac{\mu \mathrm{b}}{\mathrm{sr}}$. However, since an actual longitudinal-transverse separation was not performed, the ratio $\frac{\sigma_{L}}{\sigma_{T}}$ in Figure 108 has an assigned uncertainty of 0.2 (for low $Q^{2}$ ). The assigned uncertainty produces a larger uncertainty on the estimate of the transverse component of the cross section. Nevertheless, the overestimate of the uncertainty comes from directly comparing electroproduction and photoproduction results. This larger uncertainty is \pm 0.05 . Figure 109 shows the transverse component of the electroproduction cross section in E94-107 with inner error bars because of the uncertainties in E94-107 only, and outer error bars that include the assigned uncertainty obtained from Figure 108 in addition to the uncertainty in E94-107. 


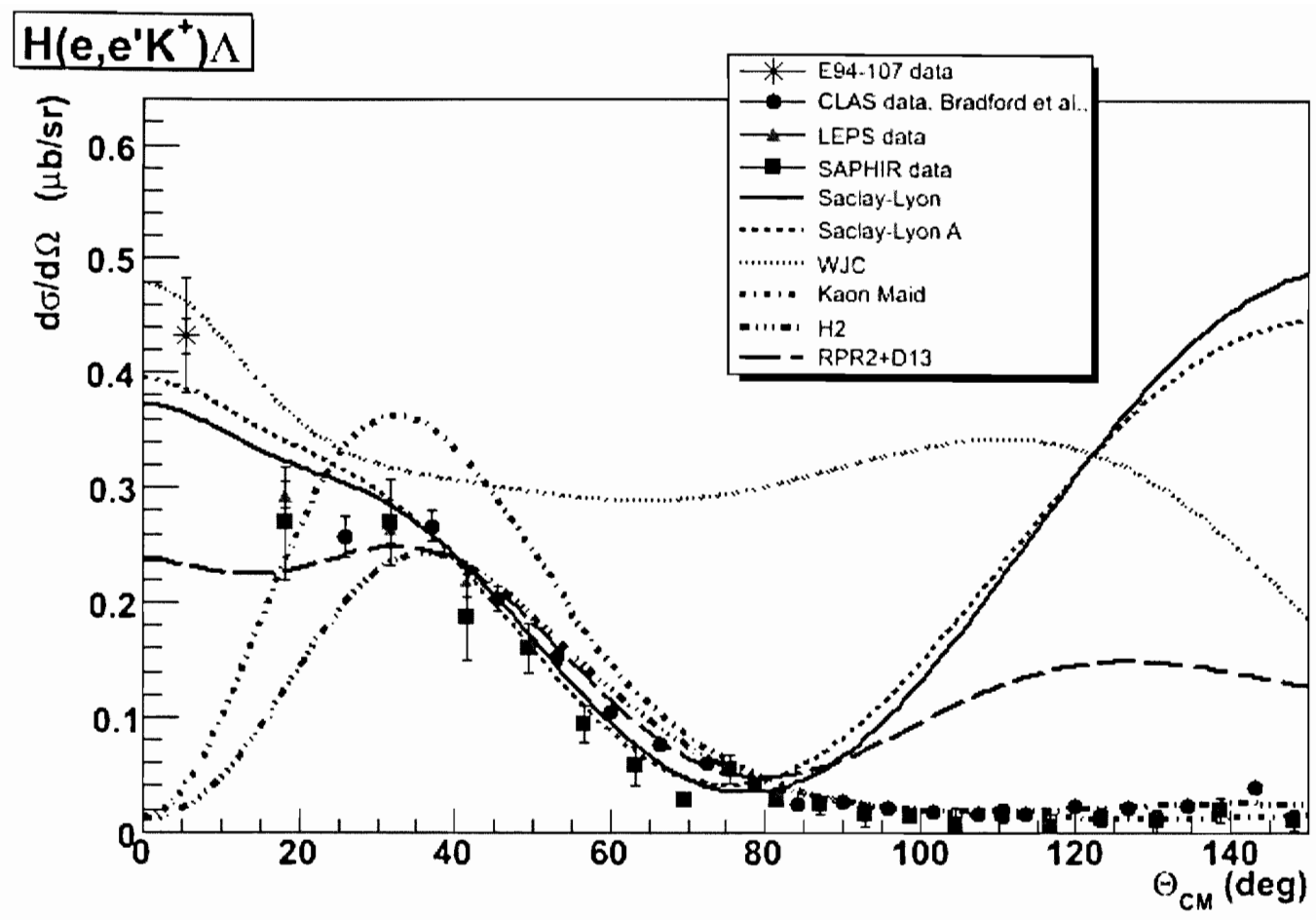

Figure 109: Estimate of the transverse component of the electroproduction cross section in E94-107 $\left(Q^{2} \sim 0.07(\mathrm{GeV} / \mathrm{c})^{2}, W=2.2 \mathrm{GeV}\right.$ and $\left.\theta_{\mathrm{CM}}=6^{\circ}\right)$ compared to photoproduction data and models, all of them with $W=2.2 \mathrm{GeV}$. Inner error bars in E94-107 estimate represent E94-107 uncertainty only. Outer error bars represent total uncertainty (E94107 uncertainty and assigned uncertainty from Figure 108). CLAS data from Bradford et al., [27].

As a conclusion for Question e), the obtained cross section for transversely polarized photons exceeds most of the predicted values by the different theoretical models and shows that the transverse component of the electroproduction cross section rises when $\theta_{\mathrm{CM}} \rightarrow 0^{0}$, except in the WJC and Saclay-Lyon A models. However, the WJC and Saclay-Lyon A models do not describe correctly the experimental measurements at higher $\theta_{\mathrm{CM}}$.

In order to answer Question d) in Section 1.5 the results taken from the data binning in $Q^{2}$ were used to find the $\frac{\Sigma^{0}}{\Lambda}$ ratios. From Table 57, it was obtained Table 60. 


\begin{tabular}{cccc}
\hline $\begin{array}{c}\text { Binning } \\
\text { in } Q^{2}\end{array}$ & $\begin{array}{c}\text { Kinematics } \\
\text { particle } \\
\text { combination }\end{array}$ & $\begin{array}{c}\text { Cross } \\
\text { section } \\
\text { ratios }\end{array}$ & Uncertainties \\
\hline Q1: & S_KIN_1/L_KIN1 & 0.4551 & 0.0475 \\
Q2: & S_KIN_1/L_KIN1 & 0.5546 & 0.0552 \\
Q3: & S_KIN_1/L_KIN1 & 0.3517 & 0.0319 \\
Q1: & S_KIN_2/L_KIN2 & 0.4643 & 0.0463 \\
Q2: & S_KIN_2/L_KIN2 & 0.5605 & 0.0662 \\
Q3: & S_KIN_2/L_KIN2 & 0.5701 & 0.0564 \\
\hline
\end{tabular}

Table 60: $\frac{\Sigma^{0}}{\Lambda}$ ratios in E94-107.

Tables 54 and 60 were used to generate Figure 110, where the solid circles correspond to KIN_1 and the solid square to KIN_2, both for different binned data in $Q^{2}$ as shown in Table 60.

From the $\frac{\Sigma^{0}}{\Lambda}$ cross-section ratios for the different kinematics listed in Table 45, the weighted by the charge (Equation (60)), average value for the $\frac{\Sigma^{0}}{\Lambda}$ cross-section ratio was determined to be $0.4761 \pm 0.0313$ (see Figure 111). The ratio would be the slightly greater for the transverse components of the cross section since the $\Sigma^{0}$ longitudinal responses appear to be smaller than the $\Lambda$. Figure 110 shows the results of $\frac{\Sigma^{0}}{\Lambda}$ crosssection ratio after binning the data in $Q^{2}$. 


\section{$\Delta: \Sigma$ Ratio vs. $\mathrm{Q}^{2}$}

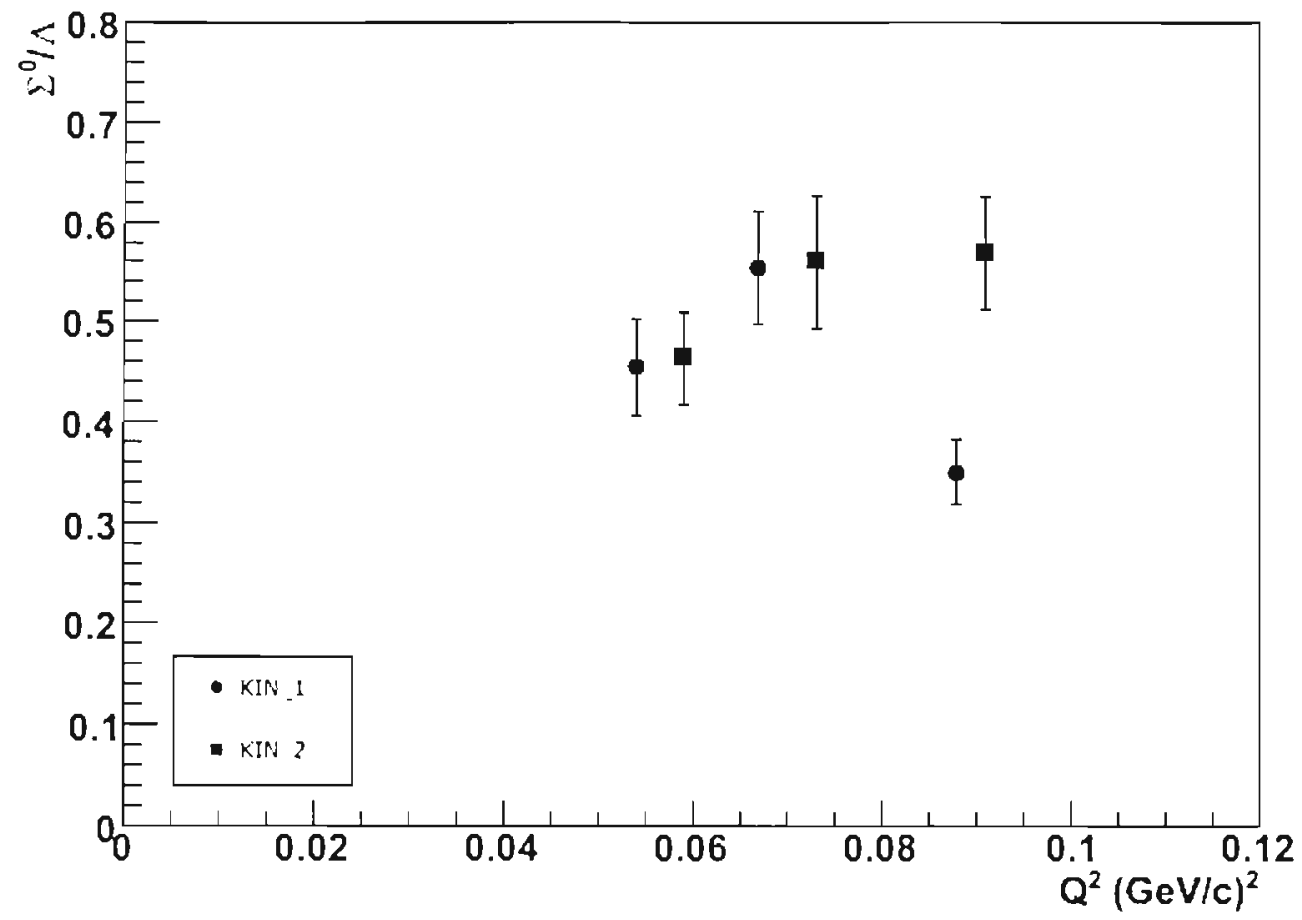

Figure 110: $\frac{\Sigma^{0}}{\Lambda}$ cross section ratio results from E94-107. Values listed in Table 60. Average variable values listed in Tables 54 and 57.

Figure 111 shows the $\frac{\Sigma^{0}}{\Lambda}$ photoproduction cross-section ratio data available from Hall B published by Bradford et al, [27]. The E94-107 $\frac{\Sigma^{0}}{\Lambda}$ electroproduction crosssection ratio has been added at a very small angle $\theta_{\mathrm{CM}}=6^{\circ}$. It can be seen that the E94107 contribution fits well on the trend of the data, which was obtained at the same invariant mass $W \approx 2.2 \mathrm{GeV}$. 


\section{CLAS Ratio of $\Sigma^{0}: \Lambda^{0}, W=2.207 \mathrm{GeV}$}

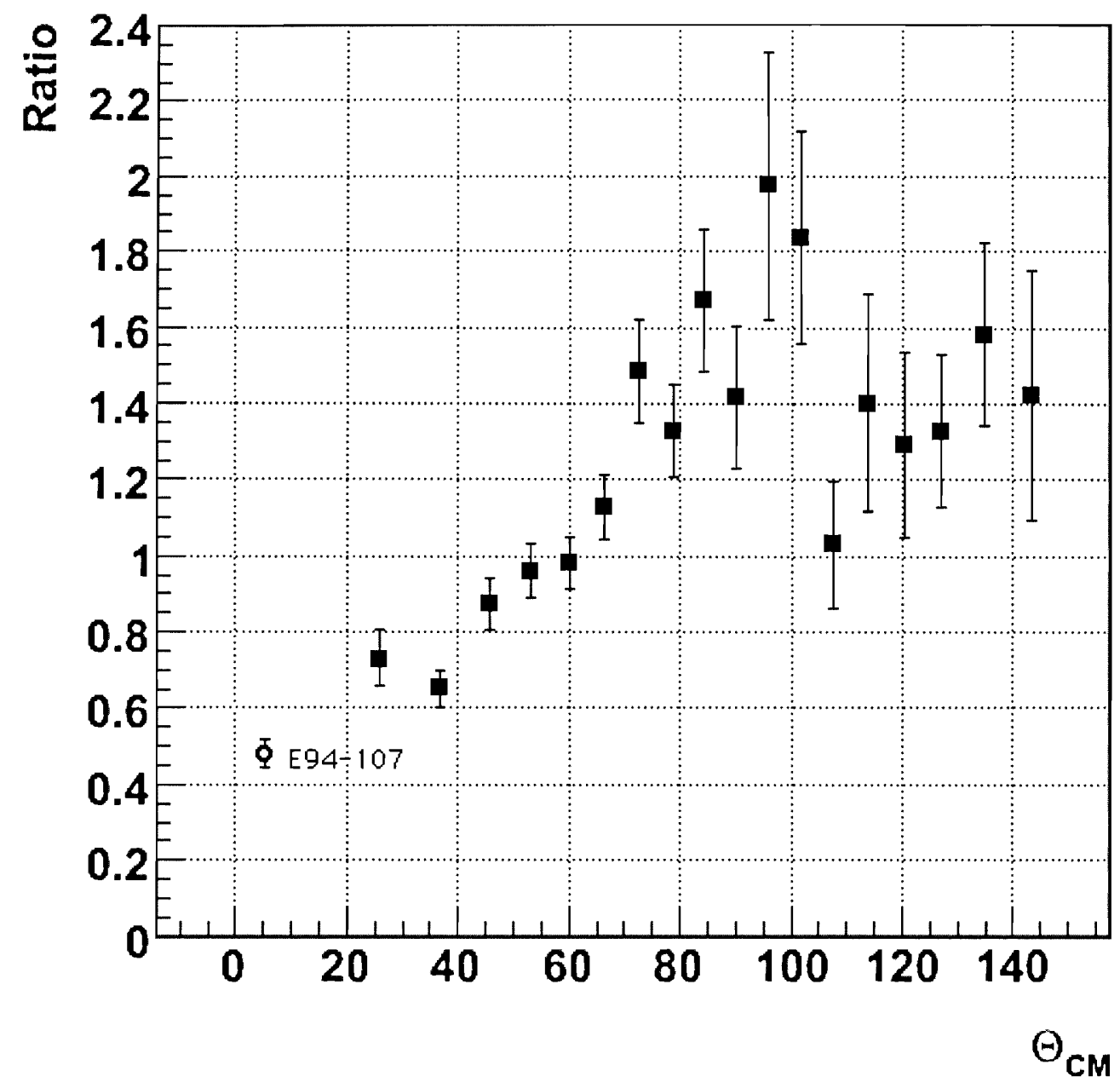

Figure 111: $\frac{\Sigma^{0}}{\Lambda}$ photoproduction cross-section ratio data from Hall B, Jlab (Bradford et al., [27]) and E94-107 contribution at $\theta_{C M}=6^{0}$, with $Q^{2}=0.07(\mathrm{GeV} / \mathrm{c})^{2}$ and $W=2.2 \mathrm{GeV}$.

Furthermore, the Kaon Maid model that includes hadronic form factors in the calculations differs from the data for $\theta_{\mathrm{CM}}$ less than $20^{\circ}$, as was shown in Figure 112 . 


\section{Kaon Maid Ratio of $\Sigma^{0}: \Lambda^{0}$ vs. $Q^{2}$}

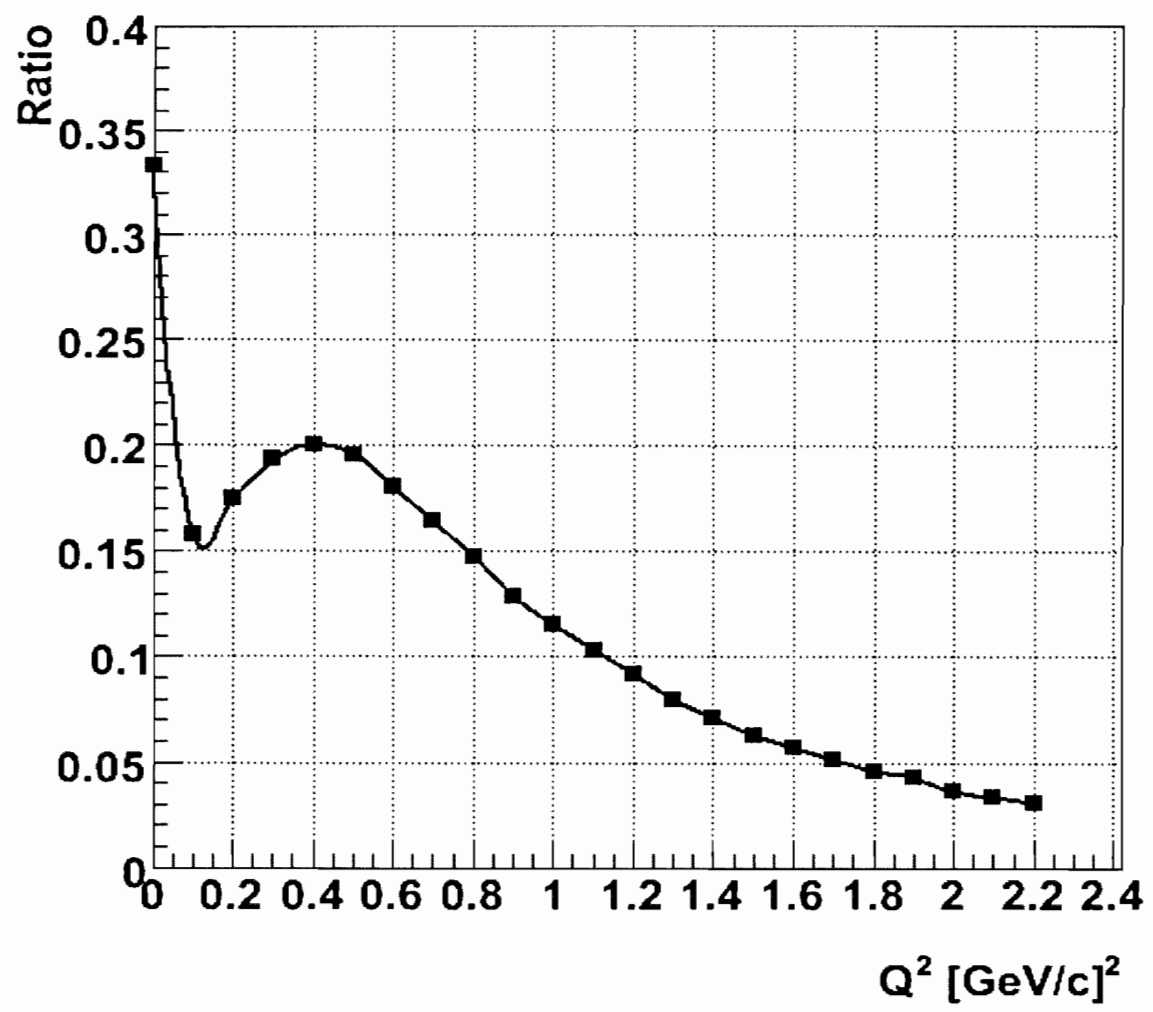

Figure 112: Kaon Maid model for $\frac{\Sigma^{0}}{\Lambda}$ cross-section ratio vs. $Q^{2}$, with $W=2.2 \mathrm{GeV}$ and $\theta_{C M}=0^{0}$.

The Kaon maid model also predicts a $\frac{\Sigma^{0}}{\Lambda}$ cross-section ratio of about 0.15 to 0.2 for $Q^{2} \approx 0.07(\mathrm{GeV} / \mathrm{c})^{2}$, which is too low (see Figure 112) compared to E94107 result (see Figure 111).

In conclusion, none of the models completely describe well the results of the photoproduction cross sections. The results of my research are important experimental measurements that will probably contribute to improve the existing theoretical models (see Section 1.5). 
Finally, it is also important to remark that in the case of the $\Sigma^{0}$ channel, the MCEEP simulation was run using a similar model as in the case of the $\Lambda$ channel. In particular, the model assumed a similar cross section dependence on the kinematic variables in averaging the cross section over the experimental acceptance. Nevertheless, the MCEEP-to-measured yield distributions for the $\Sigma^{0}$ channel showed a good agreement. Currently, although we have highlighted that there are still many adjustments to do to describe the $\Lambda$ channel mechanisms, the $\Sigma^{0}$ channel is even less known. The results of my work will probably contribute to a better understanding of the elementary reaction $\mathrm{H}\left(e, e^{\prime} K^{+}\right) \Lambda, \Sigma^{0}$ as more data become available. 
References

[1] S.R. Cotanch, R. A. Williams, and C.R. Ji, Physics Scripta 48 (1993), 217.

[2] W. Greiner and A. Shafer, Quantum Chromodynamics, Springer-Verlag, Berlin 1994.

[3] F.E. Close, Nucl.Phys. B73 (1974), 410.

[4] D. Haidt and P.M. Zerwas, European Phys. Journal C 3 (1998), no. 1-4, Particle Data Book.

[5] F. Halzen and A.D. Martin, Quarks and Leptons, John Wiley and Sons, New York, 1984.

[6] D. Griffiths, Introduction to Elementary Particle Physics, John Wiley and Sons, 1987.

[7] Povh, Rith, Scholz, Zetsche, Particles and Nuclei, Springer, 2003.

[8] J. D. Walecka, Theoretical Nuclear and Subnuclear Physics, World Scientific, 2004.

[9] A. Gal and E. Hungerford, Hypernuclear and Strange Particle Physics, Proceedings of the Eighth International Conference on Hypernuclear and Strange Particle Physics, Newport News, VA, 2003.

[10] Niculescu, G. et al. (1998). Longitudinal and transverse cross sections in the ${ }^{1} \mathrm{H}\left(e, e^{\prime} K^{+}\right) \Lambda$ reaction. Phys. Rev. Lett. 81,1805.

[11] Bebek, C.J. et al. (1977). Electroproduction of $K^{+}$meson along the virtual photon direction. Phys. Rev. D15, 594.

[12] Adelseck, R. A. \& Wright, L.E. (1998). Electromagnetic production of kaon. Phys. Rev. C38, 1965.

[13] Brauel, P. et al.(1977). Separation of $\sigma_{L}$ and $\sigma_{T}$ in $\pi^{+}$electroproduction above the resonance region. Phys. Rev. Lett. 69B, 253.

[14] Carlson, C.E. \& Milana, J. (1990). Difficulty in determining the pion form factor at high $Q^{2}$. Phys. Rev. Lett. 65,1717. 
[15] S.R. Cotanch, R. A. Williams, and C.R. Ji, Physics Review C46 (1992), no 5, 1617.

[16] David G Fayard G H Lamot and B Saghai Phys Rev C53 (1996), no 6, 2613.

[17] R. Adelseck and B. Saghai, Phys. Rev. C42 (1990), no 1, 108.

[18] T. Mart, C. Bennhold and C.E. Hyde Wright, Phys. Rev. C51 (1995), no 3, R1074.

[19] P. D. B. Collins, An Introduction to Regge Theory and High Energy Physics, Cambridge University Press, Cambridge.

[20] M. Guidal, J. M. Laget and M. Vanderhaegen, Nucl. Phys. A627 (1997), 645.

[21] P. Feller et al., Nucl. Phys. B 39, (1972), 413.

[22] O. Nachtmann, Nucl. Phys. B38, (1972), 397.

[23] O. Nachtmann, Nucl. Phys. B74, (1974), 422.

[24] F.E. Close, Nucl. Phys. B73, (1974), 410.

[25] R.M. Mohring, $\mathrm{PhD}$ thesis, A Comparison of Longitudinal and Tranverse Cross Sections in the $\mathrm{p}\left(e, e^{\prime} K^{+}\right) \Lambda$ and $\mathrm{p}\left(e, e^{\prime} K^{+}\right) \Sigma^{0}$ reactions. (1999).

[26] P. Bydzovsky, private communication.

[27] R. Bradford et al (CLAS Collaboration), Phys. Rev. C 73, 035202 (2006).

[28] K.-H. Glander et al., Eur. Phys. J. A 19, 251 (2004); nucl-ex/0308025.

[29] M. Sumihama et al (LEPS Collaboration), Nucl. Phys. A 754, 303 (2005).

[30] T. Corthals, J. Ryckenbusch, T. Van Cauteren, Phys. Rev. C 73, 045207 (2006).

[31] P. Bydzovsky and M. Sotona, Nucl. Phys. A 754, 243c (2005); nucl-th/0408039.

[32] John Alcorn et al., Basic Instrumentation for Hall A at Jefferson Lab, Nuclear Instruments and Methods in Physics Research, Section A, NIM A 522, (2004).

[33] Hall A 3-dimensional image library, Jefferson lab website. http://www.jlab.org/help/Ghelp/halla3d.html. 
[34] Y. C. Chao , P. Chevtsov, A. Day , A.P. Freyberger, R. Hicks, M. Joyce and J.-C. Denard, Energy Spread Monitoring for the JLAB Experimental Program: Synchrotron Light Interferometers, Optical Transition Radiation Monitors and Wire Scanners.

http://www1.jlab.org/Ul/publications/documents/ACF1B27.pdf.

[35] P.E. Ulmer, I. Karabekov, A Saha, P. Bertin, P. Vernin, JLab internal report, CEBAF-TN-94-001 (2001).

[36] P. Chevtsov, A. Day, A.P. Freyberger, R. Hicks, Synchrotron Light Interferometry at Jefferson Lab, Proceedings of EPAC 2004, Lucerne, Switzerland.

[37] K. Unser, IEEE Transactions on Nuclear Science, Vol. NS-28, No.3, June 1981.

[38] J.H Mitchell et al., Hall A Experimental Equipment Operations Manual. http//hallaweb.jlab.org/document/OPMAN/.

[39] J. P. Chen, Jefferson Lab, Hall A, Cryotarget System.

http://hallaweb.jlab.org/equipment/targets/cryotargets/Halla_tgt.html

[40] F. Garibaldi et al., Nuclear Instruments and Methods in Physics Research, Section A, NIM A 314 (1992),1.

[41] Hall A Hypernuclear Collaboration, A Proposal For Two Septum Magnets For Forward Angle Physics in Hall A at TJNAF, The TJNAF Technical Advisory Committee (1996).

[42] W. Bertozzi et al., Nuclear Instruments and Methods in Physics Research, Section A, NIM A 191 (1977), 957.

[43] G. Charpak, D. Rahm and H. Steiner, Nucl. Instrum. Method. 80 (1970) 13.

[44] O.K. Baker et al., Nuclear Instruments and Methods in Physics Research, Section A, NIM A 367 (1995), 92.

[45] J. Litt and R. Meunier, Ann. Rev. Nucl. Sci. 23 (1973), 1.

[46] F. Garibaldi et al., Nuclear Instruments and Methods in Physics Research, Section A, NIM A 496 (2003), 305-314.

[47] M. Iodice et al., Nuclear Instruments and Methods in Physics Research, Section A, NIM A 411 (1998), 223-237.

[48] Lingyan Zhu(MIT), Particle Identification with Aerogel Cherenkov Detector 


\section{A1/A2, For JLAB-FIU-MIT-PHOTONIS.}

[49] L. Lagamba et al., Silica aerogel threshold Cherenkov counters for the JLab Hall A spectrometers: improvements and proposed modifications, Nuclear Instruments and Methods in Physics Research, Section A, NIM A 471 (2001), 325-332.

[50] E. Cisbani et al., RICH detector at Jefferson Lab, design, performance and physics results, JLAB-PHY-05-485 (DOE/ER/40150-4158).

[51] F. Cusanno et al., Nuclear Instruments and Methods in Physics Research Section A: Accelerators, Spectrometers, Detectors and Associated Equipment.Volume 525, Issues 1-2, 1 June 2004, Pages 163-167.

[52] D. Abbot et al ., The CODA system and its Performance in the First Online Experiments at CEBAF, Proceedings of the 1995 IEEE Conference on Real Time Computing Applications in Nuclear, Particle and Plasma Physics, 147-151 (May 1995).

[53] Robert Michaels, Trigger Programming for Hall A spectrometers, hallaweb:jlab.org/equipment/daq/daq_trig.html.

[54] E. Offerman et al:, ESPACE: Event Scanning Programm for Hall A Collaboration Experiments, v.2.9B, (2000).

[55] N. K. Liyanage, PhD thesis, Massachusetts Institute of Technology, (1999).

[56] Jefferson Lab Hall A ESPACE User Guide, http://wwww.jlab.org/espace/docs.html.

[57] Y. Qiang, PhD thesis, Appendix A, Massachusetts Institute of Technology (2007).

[58] F. Cusanno, PhD thesis, Appendix B, Universit'a degli Studi Roma Tre. (2004).

[59] K.G. Fissum et al., JLAB-TN-00-016, (2000).

[60] R.J. Abrams et al., Phys. Rev. D4, (1971).

[61] R. Hausmann and W. Weise, Z. Phys. A 324, 355. (1986).

[62] J. Reinhold, private communication, (2008).

[63] P. Markowitz, private communication, (2009).

[64] P. Markowitz, private communication, (2006). 
[65] B. Michaels, private communication, (2008).

[66] Hall A log webpage; http:/hallaweb.jlab.org/adaq/log/html/logdir.html, April 2004.

[67] D.S. Armstrong et al., Target Density Fluctuations and Bulk Boiling in The Hall A Cryotarget, JLAB-TN-03-017 (2003).

[68] P. Markowitz, Private communications, (2007).

[69] High Resolution Spectroscopy of ${ }_{\Lambda}^{16} N$ by Electroproduction F. Cusanno, ... A. Acha et al., Submitted to Phys. Rev. Lett., arXiv:0810.3853v1 [nucl-ex] (2008).

[70] B. Raue, Private communications, (2009).

[71] P. Markowitz, Private communications, (2006).

[72] P.E. Ulmer, MCEEP: Monte Carlo Simulation for electro-nuclear coincidence experiments, version 3.9 (2009).

[73] T. Mart and C. Bennhold, Phys. Rev. C61 (2000).

[74] A. Picklesimer and J. Van Orden, Phys. Rev. C40, 290 (1989).

[75] D.M. Koltenuk, Ph.D. Thesis, University of Pennsylvania,(1999).

[76] P. Brauel et al., Z. Phys. Rev. C3,101, (1979).

[77] J. Cha, PhD Dissertation, page 163, Hampton University (2000). 
A Kaon absorption program (by J. Reinhold).

program absorb

implicit none

real siga( $(0: 20)$, NovrN, NovrNO(0:20), R, Ns, Rabs $(0: 20)$, p, dens, A, t, Ntar, integer $\mathrm{i}$

real sigKA

c write $\left(*,{ }^{*}\right)$ 'momenta, absorp coef, absorp coef Reinhold' write $\left(*,{ }^{*}\right)$ 'momenta, absorp coef'

do $p=1 ., 2.6,0.01$

c $\quad /^{*}$ function calls for the various materials for a given momemtum */

C $\quad I^{*}$ first do hydrogen target */

$A=1.01$

$\mathrm{t}=4.00$

dens $=0.0723$

!/* atomic weight, hydrogen */

$\operatorname{siga}(0)=0.804^{*}\left(\mathrm{p}^{* *}(-0.99)\right) * 26.2^{*}\left(\mathrm{~A}^{* *} 0.79\right) ! /^{*}$ cross section in mb*/

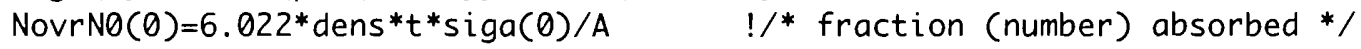

$\operatorname{Rabs}(\theta)=6.022 \mathrm{E}-4 *$ dens*t*sigKA(1., p)/A

C /* second: target walls second */ !/* aluminum */

$A=26.98$

$t=0.0102$

dens $=2.70$

$\operatorname{siga}(1)=0.804 *(p * *(-0.99)) * 26.2 *(A * 0.79)$

NovrNO(1) $=6.022 *$ dens*t*siga(1)/A

$\operatorname{Rabs}(1)=6.022 \mathrm{E}-4 *$ dens*t*sigKA(27, , p $) / A$

c ! third: VDC detectors

C !/* kevlar (approx)*/

$A=(5 . * 12.01+4 . * 1.01+2 . * 16.00) / 11.0$

$t=0.0381$

dens $=0.74$

$\operatorname{siga}(3)=0.804 *\left(p^{* *}(-0.99)\right) * 26.2 *(A * 0.79)$

NovrNO(3) $=6.022 *$ dens*t*siga( 3$) / A$

Ntar $=6.022 \mathrm{E}-4 *$ dens*t/(5.*12.01+4.*1.01+2.*16.00)

$\operatorname{Rabs}(3)=\operatorname{Ntar} *\left(5 . * \operatorname{sigKA}(12,, p)+4 .{ }^{*} \operatorname{sigKA}(1,, p)+2 .{ }^{*} \operatorname{sigKA}(16, p)\right)$

c $\quad I^{*}$ wire chambers (2 each), fourth */

c $\quad ! / *$ tungsten */

$A=183.85$

$t=0.0000212 * 2 .+0.00085 * 2$

dens $=19.30$

$\operatorname{siga}(4)=0.804 *(p * *(-0.99)) * 26.2 *(A * * 0.79)$

NovrNO(4) $=6.022 *$ dens*t*siga $(4) / A$ 
Ntar $=6.022 \mathrm{E}-4 *$ dens $* \mathrm{t} / \mathrm{A}$

$\operatorname{Rabs}(4)=N \operatorname{tar}^{*} \operatorname{sigKA}(184, \mathrm{p})$

c ! !* cathode (mylar) fifth */

$A=(5 . * 12.01+4 . * 1.01+2 . * 16.00) / 11.0$

$\mathrm{t}=0.0044 * 2$.

dens $=1.39$

$\operatorname{siga}(5)=0.804 *\left(p^{* *}(-0.99)\right) * 26.2 *(A * * 0.79)$

NovrNO(5) $=6.022 *$ dens*t*siga $(5) / A$

Ntar $=6.022 \mathrm{E}-4 *$ dens*t/(5.*12.01+4.*1.01+2.*16.00)

$\operatorname{Rabs}(5)=\operatorname{Ntar} *\left(5 .{ }^{*} \operatorname{sigKA}(12, p)+4 .{ }^{*} \operatorname{sigKA}(1,, p)+2 .{ }^{*} \operatorname{sigKA}(16, p)\right)$

C ! I/* Ar-ethane air sixth */

$A=(39.95+(2 . * 12.01+6 . * 1.01) / 8) /$.2 .

$\mathrm{t}=10.16 * 2$.

dens $=((1.356+1.780) / 2) * 1 . E-$.

$\operatorname{siga}(6)=0.804 *\left(p^{* *}(-0.99)\right) * 26.2 *(A * * 0.79)$

NovrNO(6) $=6.022 *$ dens*t*siga $(6) / A$

Ntar $=6.022 \mathrm{E}-4 *$ dens*t/(39.95 $+2 . * 12.01+6 . * 1.01)$

$\operatorname{Rabs}(6)=\operatorname{Ntar} *\left(\operatorname{sigKA}(40 ., p)+2 .{ }^{*} \operatorname{sigKA}(12 ., p)+6 .{ }^{*} \operatorname{sigKA}(1 ., p)\right)$

c $\quad$ * scintillators ( 2 each), seventh */

C ! /* mylar */

$A=(5 . * 12.01+4 . * 1.01+2 . * 16.00) / 11.0$

$\mathrm{t}=.00254 * 2$.

dens $=1.39$

$\operatorname{siga}(7)=0.804 *\left(p^{* *}(-0.99)\right) * 26.2 *(A * 0.79)$

NovrNO(7) $=6.022 *$ dens*t*siga( 7$) / A$

Ntar $=6.022 \mathrm{E}-4 *$ dens*t/(5.*12.01+4.*1.01+2.*16.00)

$\operatorname{Rabs}(7)=\operatorname{Ntar} *\left(5 . * \operatorname{sigKA}(12, p)+4 . * \operatorname{sigKA}(1,, p)+2 .{ }^{*} \operatorname{sigKA}(16 ., p)\right)$

C !eight !/* aluminum */

$A=26.98$

$\mathrm{t}=0.0000480 * 2$.

dens $=2.70$

$\operatorname{siga}(8)=0.804 *\left(p^{* *}(-0.99)\right) * 26.2 *(A * 0.79)$

NovrNO(8) $=6.022 *$ dens*t*siga $(8) / A$

$\mathrm{Ntar}=6.022 \mathrm{E}-4 *$ dens $* \mathrm{t} / \mathrm{A}$

$\operatorname{Rabs}(8)=N \operatorname{tar}^{*} \operatorname{sigKA}(27, \mathrm{p})$

C !nineth ! /* polystyrene */

$A=(1.01+12.01) / 2$.

$\mathrm{t}=1.125 * 2$.

dens $=1.032$

$\operatorname{siga}(9)=0.804 *\left(p^{* *}(-0.99)\right) * 26.2 *(A * 0.79)$

NovrNO(9) $=6.022 *$ dens*t*siga( $(9) / A$

Ntar $=6.022 \mathrm{E}-4 *$ dens $* t /(12.01+1.01)$ 


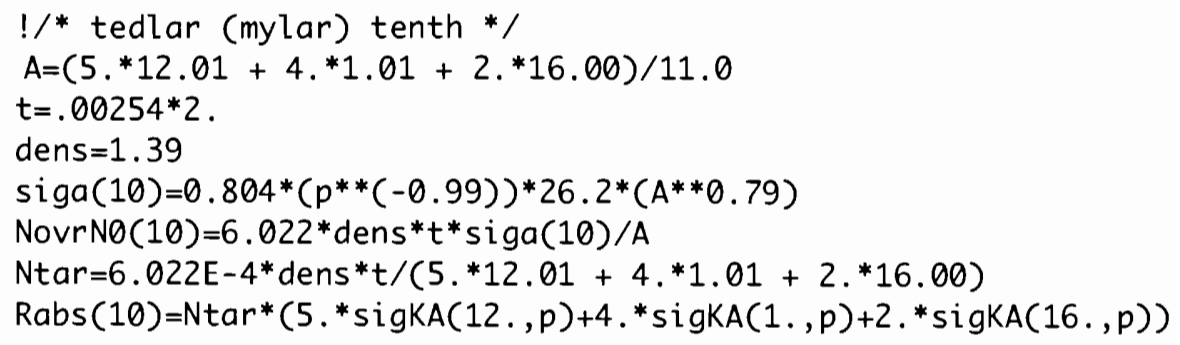

c /* AEROGEL A1 Cherenkokv, eleventh */ !/*AEROGEL A1 */ $A=(12.01+2 . * 16.00) / 3$.

$t=9.00$

dens $=0.074$

$\operatorname{siga}(11)=0.804 *\left(p^{* *}(-0.99)\right) * 26.2 *\left(A^{* * 0} 0.79\right)$

NovrNO(11) $=6.022 *$ dens $* t * \operatorname{siga}(11) / A$

Ntar $=6.022 \mathrm{E}-4 *$ dens*t/(12.01+2.*16.00)

$\operatorname{Rabs}(11)=\operatorname{Ntar}^{*}(\operatorname{sigKA}(12,, p)+2 . * \operatorname{sigKA}(16, p))$

C I* AEROGEL A2 Cherenkov, twelveth */ !/*AEROGEL A2 */

$A=(12.01+2 . * 16.00) / 3$.

$t=5.00$

dens $=0.074$

$\operatorname{siga}(12)=0.804 *\left(p^{* *}(-0.99)\right) * 26.2 *\left(A^{* * 0} .79\right)$

NovrNO(12) $=6.022 *$ dens*t*siga $(12) / A$

Ntar $=6.022 \mathrm{E}-4 *$ dens*t/(12.01+2.*16.00)

$\operatorname{Rabs}(12)=\operatorname{Ntar}^{*}\left(\operatorname{sigKA}(12,, p)+2 .{ }^{*} \operatorname{sigKA}(16, p)\right)$

c ! !* kevlar (approx) thirteenth*/

$A=(5 . * 12.01+4 . * 1.01+2 . * 16.00) / 11.0$

$t=0.0381 * 2$

dens $=0.74$

$\operatorname{siga}(13)=0.804 *\left(p^{* *}(-0.99)\right) * 26.2 *\left(A^{* * 0} .79\right)$

NovrNO(13) $=6.022 *$ dens*t*siga $(13) / A$

Ntar $=6.022 \mathrm{E}-4 *$ dens*t/(5.*12.01+4.*1.01+2.*16.00)

$\operatorname{Rabs}(13)=\operatorname{Ntar} *\left(5 .{ }^{*} \operatorname{sigKA}(12,, p)+4 .{ }^{*} \operatorname{sigKA}(1 ., p)+2 .{ }^{*} \operatorname{sigKA}(16 ., p)\right)$

C ! RICh

C !Neoceram+quartz $\mathrm{SiO2}$

$A=(28.086+2 . * 16.00) / 3$.

$t=0.90$

dens $=2.20$

$\operatorname{siga}(14)=0.804 *\left(p^{* *}(-0.99)\right) * 26.2 *(A * 0.79)$

NovrNO(14) $=6.022 *$ dens*t*siga(14) $/ \mathrm{A}$

Ntar $=6.022 \mathrm{E}-4 *$ dens*t $/\left(28.086+2 .{ }^{*} 16.00\right)$

$\operatorname{Rabs}(14)=\operatorname{Ntar}^{*}\left(\operatorname{sigKA}(28,, p)+2 .{ }^{*} \operatorname{sigKA}(16, p)\right)$ 
$A=(6 . * 12.01+14 . * 18.998) / 20$.

$t=1.5$

dens $=1.68$

$\operatorname{siga}(15)=0.804 *\left(p^{* *}(-0.99)\right) * 26.2 *\left(A^{* * 0} 0.79\right)$

NovrNO(15) $=6.022 *$ dens*t*siga $(15) / A$

Ntar $=6.022 \mathrm{E}-4 *$ dens*t/(6.*12.01+14.*18.998)

$\operatorname{Rabs}(15)=\operatorname{Ntar}^{*}\left(6 . * \operatorname{sigKA}(12,, p)+14 .{ }^{*} \operatorname{sigKA}(19 ., p)\right)$

C !methane

$A=(12.01+4 . * 1.01) / 5$.

$\mathrm{t}=10.0$

dens $=0.0004$

$\operatorname{siga}(16)=0.804 *\left(p^{* *}(-0.99)\right) * 26.2 *(A * 0.79)$

NovrNO(16) $=6.022 *$ dens*t*siga $(16) / A$

Ntar $=6.022 \mathrm{E}-4 *$ dens*t/(12.01+4.*1.01)

$\operatorname{Rabs}(16)=\operatorname{Ntar} *(\operatorname{sigKA}(12, p)+4 . * \operatorname{sigKA}(1, p))$

C !rohacell:polymethacrylimide aprox ! -C2H7N02- rich electronics $A=\left(2 .{ }^{*} 12.01+7 .{ }^{*} 1.01+1 .{ }^{*} 14.007+2 .{ }^{*} 16.00\right) / 12$.

$t=4.0$

dens $=0.03$

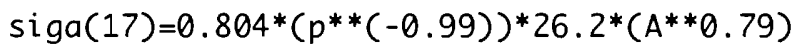

NovrNO(17) $=6.022 *$ dens $* t^{*}$ siga(17) $/ A$

Ntar $=6.022 \mathrm{E}-4 *$ dens*t $/\left(2 .{ }^{*} 12.01+7 .{ }^{* 1.01}+1 .{ }^{* 14.007}+2 . * 16.00\right)$

$\operatorname{Rabs}(17)=\operatorname{Ntar} *\left(2 .{ }^{*} \operatorname{sigKA}(12, \mathrm{p})+7 . * \operatorname{sigKA}(1 ., \mathrm{p})\right)$

$\operatorname{Rabs}(17)=\operatorname{Rabs}(17)+\operatorname{Ntar} *\left(1 .{ }^{*} \operatorname{sigKA}(14 ., p)+2 .{ }^{*} \operatorname{sigKA}(16 ., p)\right)$

c !18th !/* aluminum rich */ rich electronics

$A=26.98$

$t=0.1$

dens $=2.70$

$\operatorname{siga}(18)=0.804 *\left(p^{* *}(-0.99)\right) * 26.2 *\left(A^{* * 0} 0.79\right)$

NovrNO(18) $=6.022 *$ dens*t*siga $(18) / A$

Ntar $=6.022 \mathrm{E}-4^{*}$ dens*t/A

$\operatorname{Rabs}(18)=N \operatorname{tar}^{*} \operatorname{sigKA}(27, \mathrm{p})$

C /*air, ninth */ !/* air*/

$A=(0.80 * 14.01+0.20 * 16.00)$

$t=25.0$

dens $=1.29 \mathrm{E}-3$

$\operatorname{siga}(19)=0.804 *\left(p^{* *}(-0.99)\right) * 26.2 *\left(A^{* * 0} .79\right)$

NovrNO(19) $=6.022 *$ dens*t*siga $(19) / A$

Ntar $=6.022 \mathrm{E}-4 *$ dens*t/(8.*14.01+2.*16.00)

$\operatorname{Rabs}(19)=\operatorname{Ntar}^{*}\left(8 .{ }^{*} \operatorname{sigKA}(14,, p)+2 .{ }^{*} \operatorname{sigKA}(16, p)\right)$

C $\quad / *$ now sum the cross sections and $\mathrm{N} / \mathrm{NO} *$ /

NovrN $=0.0$ 
$\mathrm{R}=0.0$

$\mathrm{Ns}=1$.

do $\mathrm{i}=0,19$

NovrN $=\operatorname{NovrN}+\operatorname{NovrNO}(i)$

$\mathrm{R}=\mathrm{R}+\mathrm{R} a b s(\mathrm{i})$

$N s=N s *(1 .-\operatorname{Rabs}(i))$

enddo

write $(*, *)$ p, NovrN*1E-4

enddo

stop

end

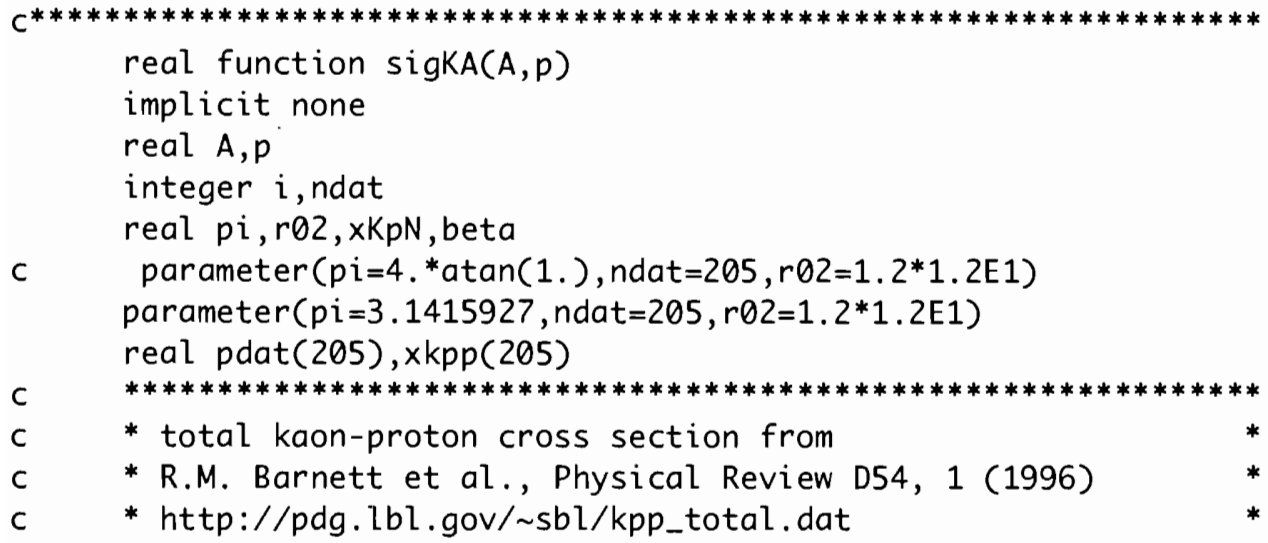
data pdat/

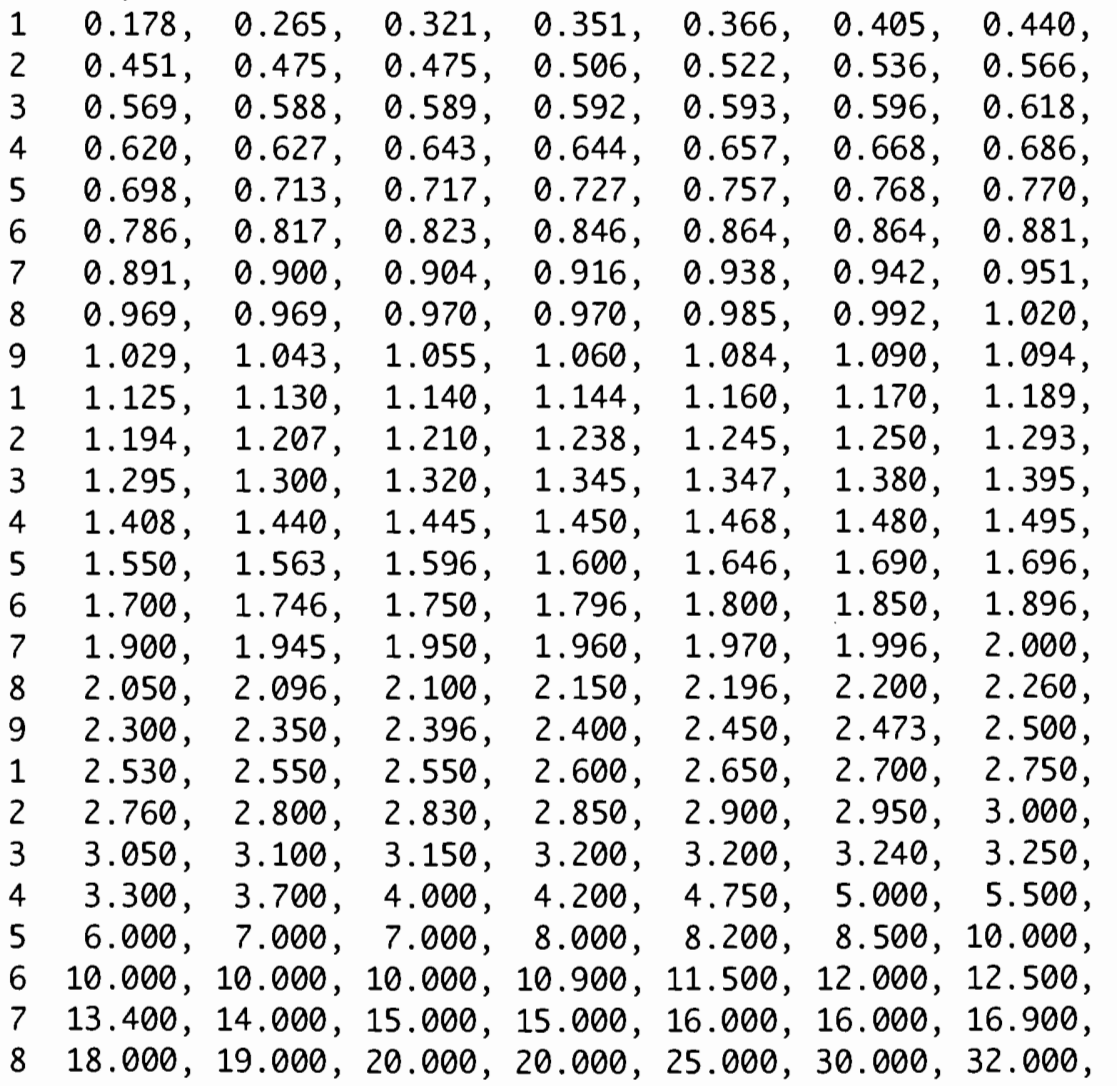


$935.000,35.000,40.000,42.500,45.000,50.000,50.000$, $152.200,55.000,70.000,100.000,100.000,120.000,147.000$, $2150.000,170.000,200.000,200.000,240.000,240.000,280.000$, $3280.000,310.000 /$

data $\mathrm{xkp} /$

$111.400,13.000,14.000,12.200,13.200,13.690,12.810$, $216.300,12.700,13.580,13.020,15.200,12.090,13.200$, $312.700,12.600,16.300,14.360,13.050,13.040,12.650$, $412.910,12.180,12.500,12.880,12.430,13.100,11.250$, $512.600,11.140,11.100,12.450,12.650,11.650,13.000$, $612.800,13.200,12.970,13.450,14.070,13.210,13.900$, $714.390,13.100,14.230,14.200,14.590,15.570,14.950$, $815.630,15.280,15.250,15.400,16.200,15.970,16.100$, $915.690,17.390,16.950,16.400,17.040,17.600,17.120$, $117.550,18.080,18.020,18.090,17.950,18.100,18.060$, $218.470,19.850,18.580,18.110,18.540,20.710,18.440$, $318.610,17.900,19.330,18.440,18.270,18.640,18.270$, $417.970,18.100,18.040,18.200,17.940,18.040,17.930$, $517.700,17.660,17.750,17.710,17.860,17.500,17.850$, $617.730,17.800,17.830,17.800,17.980,17.770,17.810$, $717.790,17.410,17.750,19.400,16.900,17.600,17.630$, $817.720,17.510,17.560,17.570,17.540,17.600,17.100$, $917.440,17.520,17.550,17.560,17.480,17.250,17.490$, $117.470,17.100,17.440,17.500,17.470,17.410,17.410$, $217.410,17.400,16.700,17.300,17.340,17.300,17.190$, $317.140,17.080,17.150,17.130,17.130,17.130,17.500$, $417.140,21.000,17.600,17.100,21.300,17.200,17.900$, $517.000,17.200,18.400,17.300,17.200,18.700,17.300$, $617.200,17.300,18.800,18.100,19.000,17.300,18.300$, $717.500,17.400,17.310,18.500,17.100,17.000,18.800$, $817.100,17.300,17.420,17.500,17.680,17.720,18.400$, $917.800,17.820,18.050,17.910,17.880,18.060,18.370$, $118.280,18.170,18.520,18.700,18.880,19.140,19.520$, $219.360,19.640,19.910,19.900,20.220,20.280,20.450$, $320.640,20.670 /$

c

c *** find $\mathrm{Kp} x$-section at momentum $\mathrm{p} * * *$

c do $i=1$, ndat

$i=1$

do while(i.lt.ndat)

if $(p \operatorname{dat}(i)$.eq.p) then

$x \mathrm{KpN}=x \mathrm{kpp}(\mathrm{i})$

$i=$ ndat +1

endif

if (pdat (i).gt.p)then

\&

$x K p N=x k p p(i-1)+(\operatorname{xkpp}(i)-x k p p(i-1)) *(p-\operatorname{pdat}(i-1)) /$ $(\operatorname{pdat}(i)-\operatorname{pdat}(i-1))$

endif

$i=$ ndat +1

$i=i+1$ 
enddo

if $(\operatorname{abs}(A-1.) \cdot$ tt.0.1) then

else

$\operatorname{sigKA}=x K p N$

c $\quad * * *$ calculate KA $x$-section using the eikonal approximation of

c $\quad * * *$ W. Weise, Il Nuovo Cimento 102A(1989)265.

C *** J.Reinhold, 5/27/98:

c *** beta determined by fit to $\mathrm{K}-12 \mathrm{C}$ absorption data from:

c *** R.J.Abrams et al., Phys.Rev.D 4(1971)3235.

bet $a=0.82119-0.11274^{*} p$

$\operatorname{sigKA}=A^{*} x K_{p N}^{*}(1 .-(3 . / 8) *.(x K p N /(p i * r 02)) *$

\&. (1. -beta*beta)*A**(1./3.))

endif

return

end 
B Scintillators' efficiencies measurement details.

\begin{tabular}{|c|c|c|c|c|c|c|c|}
\hline Run Number & psl & ps2 & ps3 & ps 4 & ps 5 & ps6 & ps7 \\
\hline \multicolumn{8}{|l|}{ KIN_1 } \\
\hline 2485 & 12000 & 1200 & 4150 & 900 & 3 & 1200 & 1 \\
\hline 2486 & 12000 & 1200 & 4150 & 900 & 3 & 1200 & 1 \\
\hline 2487 & 12000 & 1200 & 4150 & 900 & 3 & 1200 & 1 \\
\hline 2488 & 12000 & 1200 & 4150 & 900 & 2 & 1200 & 1 \\
\hline 2489 & 12000 & 1200 & 4150 & 900 & 2 & 1200 & 1 \\
\hline 2490 & 12000 & 1200 & 4150 & 900 & 2 & 1200 & 1 \\
\hline 2491 & 12000 & 1200 & 4150 & 900 & 2 & 1200 & 1 \\
\hline 2492 & 12000 & 1200 & 4150 & 900 & 2 & 1200 & 1 \\
\hline \multicolumn{8}{|l|}{ KIN 2} \\
\hline 2496 & 24000 & 1200 & 4150 & 900 & 3 & 1200 & 1 \\
\hline 2497 & 24000 & 1200 & 4150 & 900 & 3 & 1200 & 1 \\
\hline 2498 & 24000 & 1200 & 4150 & 900 & 3 & 1200 & 1 \\
\hline 2499 & 24000 & 1200 & 4150 & 900 & 3 & 1200 & 1 \\
\hline 2500 & 24000 & 1200 & 4150 & 900 & 3 & 1200 & 1 \\
\hline 2501 & 24000 & 1200 & 4150 & 900 & 3 & 1200 & 1 \\
\hline 2503 & 24000 & 1200 & 4150 & 900 & 3 & 1200 & 1 \\
\hline 2504 & 24000 & 1200 & 4150 & 900 & 3 & 1200 & 1 \\
\hline 2505 & 24000 & 1200 & 4150 & 900 & 3 & 1200 & 1 \\
\hline 2506 & 24000 & 1200 & 4150 & 900 & 3 & 1200 & 1 \\
\hline 2507 & 24000 & 1200 & 4150 & 900 & 3 & 1200 & 1 \\
\hline 2508 & 24000 & 1200 & 4150 & 900 & 3 & 1200 & 1 \\
\hline \multicolumn{8}{|l|}{$\mathrm{KIN} \_3$} \\
\hline 2509 & 24000 & 1200 & 4150 & 900 & 3 & 1200 & 1 \\
\hline 2510 & 24000 & 1200 & 4150 & 900 & 3 & 1200 & 1 \\
\hline 2511 & 24000 & 1200 & 4150 & 900 & 3 & 1200 & 1 \\
\hline 2512 & 24000 & 1200 & 4150 & 900 & 3 & 1200 & 1 \\
\hline 2513 & 24000 & 1200 & 4150 & 900 & 3 & 1200 & 1 \\
\hline 2514 & 24000 & 1200 & 4150 & 900 & 3 & 1200 & 1 \\
\hline 2515 & 24000 & 1200 & 4150 & 900 & 3 & 1200 & 1 \\
\hline 2516 & 24000 & 1200 & 4150 & 900 & 3 & 1200 & 1 \\
\hline 2517 & 24000 & 1200 & 4150 & 900 & 3 & 1200 & 1 \\
\hline 2518 & 24000 & 1200 & 4150 & 900 & 3 & 1200 & 1 \\
\hline 2519 & 24000 & 1200 & 4150 & 900 & 3 & 1200 & 1 \\
\hline 2520 & 24000 & 1200 & 4150 & 900 & 3 & 1200 & 1 \\
\hline 2521 & 24000 & 1200 & 4150 & 900 & 3 & 1200 & 1 \\
\hline
\end{tabular}

Table 61: Trigger prescale factors. 


\begin{tabular}{|c|c|c|c|c|c|c|c|}
\hline Run Number & $\mathrm{Tl}$ & $\mathrm{T} 2$ & $\mathrm{~T} 3$ & $\mathrm{~T} 4$ & TS & T6 & $\mathrm{T} 7$ \\
\hline \multicolumn{8}{|l|}{ KIN_1 } \\
\hline 2485 & 17821 & 3913 & 18281 & 4599 & 777095 & 9663 & 273646 \\
\hline 2486 & 17646 & 3891 & 18098 & 4565 & 776592 & 9528 & 273042 \\
\hline 2487 & 17656 & 3939 & 18155 & 4625 & 776468 & 9580 & 273438 \\
\hline 2488 & 13411 & 2982 & 13831 & 3444 & 875464 & 7264 & 202469 \\
\hline 2489 & 13475 & 2979 & 13747 & 3480 & 874590 & 7403 & 203680 \\
\hline 2490 & 13631 & 2981 & 14018 & 3544 & 875133 & 7577 & 202925 \\
\hline 2491 & 13126 & 2890 & 13386 & 3390 & 875909 & 7041 & 203265 \\
\hline 2492 & 13479 & 2946 & 13727 & 3524 & 875026 & 7257 & 203867 \\
\hline \multicolumn{8}{|l|}{$\underline{\mathrm{KIN}} 2$} \\
\hline 2496 & 6577 & 3213 & 14091 & 3604 & 779226 & 7401 & 257549 \\
\hline 2497 & 6881 & 3371 & 14727 & 3829 & 806462 & 7727 & 265597 \\
\hline 2498 & 6914 & 3307 & 14798 & 3847 & 806804 & 7856 & 267715 \\
\hline 2499 & 6784 & 3336 & 14598 & 3784 & 805625 & 7688 & 267277 \\
\hline 2500 & 6892 & 3358 & 14731 & 3814 & 806086 & 7751 & 268173 \\
\hline \multirow[t]{2}{*}{2501} & 5941 & 2872 & 12747 & 3269 & 717090 & 6710 & 237256 \\
\hline & 6581 & 3231 & 14054 & 3621 & 777492 & 7496 & 259602 \\
\hline 2503 & 46 & 46 & 46 & 46 & 46 & 46 & 46 \\
\hline 2504 & 6795 & 3294 & 14556 & 3799 & 807394 & 7684 & 267053 \\
\hline 2505 & 6915 & 3350 & 14659 & 3817 & 806885 & 7763 & 268460 \\
\hline 2506 & 2740 & 1345 & 5843 & 1517 & 323935 & 3056 & 107991 \\
\hline 2507 & 6799 & 3326 & 14550 & 3769 & 805201 & 7751 & 267190 \\
\hline 2508 & 6733 & 3288 & 14674 & 3830 & 805526 & 7734 & 267515 \\
\hline \multicolumn{8}{|l|}{ KIN_3 } \\
\hline 2509 & 7811 & 3797 & 14930 & 3674 & 799028 & 7924 & 273103 \\
\hline 2510 & 7847 & 3773 & 15115 & 3653 & 798667 & 8049 & 273161 \\
\hline 2511 & 7619 & 3781 & 14655 & 3591 & 799770 & 7923 & 271664 \\
\hline 2512 & 7810 & 3830 & 14989 & 3694 & 798016 & 7981 & 273985 \\
\hline 2513 & 7819 & 3791 & 15033 & 3691 & 799469 & 8085 & 273578 \\
\hline 2514 & 7742 & 3760 & 14861 & 3644 & 798998 & 7953 & 272847 \\
\hline 2515 & 7733 & 3826 & 14918 & 3692 & 799050 & 7951 & 273484 \\
\hline 2516 & 7633 & 3811 & 14694 & 3650 & 798514 & 7856 & 274656 \\
\hline 2517 & 7697 & 3796 & 14817 & 3639 & 798433 & 7960 & 273477 \\
\hline 2518 & 7656 & 3772 & 14843 & 3583 & 799360 & 7852 & 273450 \\
\hline 2519 & 7688 & 3730 & 14824 & 3632 & 798556 & 7949 & 273963 \\
\hline 2520 & 7882 & 3816 & 15051 & 3650 & 796565 & 8087 & 274800 \\
\hline 2521 & 7797 & 3757 & 15025 & 3696 & 797052 & 8058 & 274063 \\
\hline
\end{tabular}

Table 62: Trigger events. 


\begin{tabular}{|c|c|c|c|c|c|c|}
\hline Run Number & $E$ eff & $\mathrm{H}$ eff & Scint eff & Uncert. E & Uncert. H & Uncert. Scint \\
\hline \multicolumn{7}{|l|}{ KIN_1 } \\
\hline 2485 & 0.97877 & 0.95606 & 0.93576 & 0.00001 & 0.00002 & 0.00002 \\
\hline 2486 & 0.97868 & 0.95594 & 0.93556 & 0.00001 & 0.00002 & 0.00002 \\
\hline 2487 & 0.97844 & 0.95553 & 0.93493 & 0.00001 & 0.00002 & 0.00002 \\
\hline 2488 & 0.97850 & 0.95645 & 0.93589 & 0.00001 & 0.00002 & 0.00003 \\
\hline 2489 & 0.97862 & 0.95590 & 0.93546 & 0.00001 & 0.00002 & 0.00003 \\
\hline 2490 & 0.97885 & 0.95595 & 0.93573 & 0.00001 & 0.00002 & 0.00003 \\
\hline 2491 & 0.97872 & 0.95579 & 0.93544 & 0.00001 & 0.00002 & 0.00003 \\
\hline 2492 & 0.97886 & 0.95520 & 0.93501 & 0.00001 & 0.00002 & 0.00003 \\
\hline \multicolumn{7}{|l|}{ KIN 2} \\
\hline 2496 & 0.97653 & 0.95569 & 0.93326 & 0.00001 & 0.00002 & 0.00003 \\
\hline 2497 & 0.97646 & 0.95497 & 0.93249 & 0.00001 & 0.00002 & 0.00003 \\
\hline 2498 & 0.97701 & 0.95503 & 0.93307 & 0.00001 & 0.00002 & 0.00003 \\
\hline 2499 & 0.97638 & 0.95513 & 0.93257 & 0.00001 & 0.00002 & 0.00003 \\
\hline 2500 & 0.97659 & 0.95516 & 0.93280 & 0.00001 & 0.00002 & 0.00003 \\
\hline 2501 & 0.97678 & 0.95561 & 0.93342 & 0.00001 & 0.00003 & 0.00003 \\
\hline 2503 & 0.95239 & 0.85609 & 0.81533 & 0.00020 & 0.00065 & 0.00065 \\
\hline 2504 & 0.97671 & 0.95486 & 0.93262 & 0.00001 & 0.00002 & 0.00003 \\
\hline 2505 & 0.97672 & 0.95496 & 0.93273 & 0.00001 & 0.00002 & 0.00003 \\
\hline 2506 & 0.97642 & 0.95503 & 0.93251 & 0.00002 & 0.00004 & 0.00004 \\
\hline 2507 & 0.97650 & 0.95522 & 0.93278 & 0.00001 & 0.00002 & 0.00003 \\
\hline 2508 & 0.97655 & 0.95483 & 0.93243 & 0.00001 & 0.00002 & 0.00003 \\
\hline \multicolumn{7}{|l|}{$\mathrm{K} \perp \mathrm{N}_{-} 3$} \\
\hline 2509 & 0.97660 & 0.95730 & 0.93490 & 0.00001 & 0.00002 & 0.00002 \\
\hline 2510 & 0.97684 & 0.95803 & 0.93585 & 0.00001 & 0.00002 & 0.00002 \\
\hline 2511 & 0.97613 & 0.95760 & 0.93474 & 0.00001 & 0.00002 & 0.00002 \\
\hline 2512 & 0.97639 & 0.95725 & 0.93466 & 0.00001 & 0.00002 & 0.00002 \\
\hline 2513 & 0.97666 & 0.95746 & 0.93511 & 0.00001 & 0.00002 & 0.00002 \\
\hline 2514 & 0.97662 & 0.95750 & 0.93511 & 0.00001 & 0.00002 & 0.00002 \\
\hline 2515 & 0.97619 & 0.95709 & 0.93431 & 0.00001 & 0.00002 & 0.00002 \\
\hline 2516 & 0.97599 & 0.95698 & 0.93400 & 0.00001 & 0.00002 & 0.00003 \\
\hline 2517 & 0.97627 & 0.95746 & 0.93474 & 0.00001 & 0.00002 & 0.00002 \\
\hline 2518 & 0.97629 & 0.95808 & 0.93536 & 0.00001 & 0.00002 & 0.00002 \\
\hline 2519 & 0.97665 & 0.95755 & 0.93518 & 0.00001 & 0.00002 & 0.00002 \\
\hline 2520 & 0.97669 & 0.95795 & 0.93561 & 0.00001 & 0.00002 & 0.00002 \\
\hline 2521 & 0.97680 & 0.95736 & 0.93515 & 0.00001 & 0.00002 & 0.00002 \\
\hline
\end{tabular}

Table 63: Scintillators' efficiencies and uncertainties. 
C Details about VDC analysis.

\begin{tabular}{|c|c|c|c|c|c|c|c|c|}
\hline RUN & $\begin{array}{l}\text { R4 } \\
\text { efficiency }\end{array}$ & $\begin{array}{l}\text { R3 } \\
\text { efficiency }\end{array}$ & $\begin{array}{l}\mathrm{R} 2 \\
\text { efficiency }\end{array}$ & $\begin{array}{l}\mathrm{R} 1 \\
\text { efficiency }\end{array}$ & $\begin{array}{l}\text { L4 } \\
\text { efficiency }\end{array}$ & $\begin{array}{l}\text { L3 } \\
\text { efficiency }\end{array}$ & $\begin{array}{l}\text { L2 } \\
\text { efficiency }\end{array}$ & $\begin{array}{l}\text { Ll } \\
\text { efficiency }\end{array}$ \\
\hline KIN 1 & $\begin{array}{c}\mathrm{p}=1.8665 \\
\mathrm{GeV} / \mathrm{c}\end{array}$ & & & & & & & \\
\hline $248 \overline{5}$ & 0.9922 & 0.9937 & 0.9912 & 0.9911 & 0.9901 & 0.9959 & 0.9958 & 0.9960 \\
\hline 2486 & 0.9924 & 0.9936 & 0.9913 & 0.9910 & 0.9901 & 0.9960 & 0.9958 & 0.9960 \\
\hline 2487 & 0.9922 & 0.9935 & 0.9912 & 0.9912 & 0.9902 & 0.9961 & 0.9959 & 0.9958 \\
\hline 2488 & 0.9921 & 0.9937 & 0.9913 & 0.9912 & 0.9913 & 0.9961 & 0.9960 & 0.9960 \\
\hline 2489 & 0.9923 & 0.9938 & 0.9915 & 0.9914 & 0.9911 & 0.9962 & 0.9958 & 0.9960 \\
\hline 2490 & 0.9924 & 0.9935 & 0.9913 & 0.9912 & 0.9914 & 0.9962 & 0.9961 & 0.9960 \\
\hline 2491 & 0.9924 & 0.9936 & 0.9911 & 0.9912 & 0.9913 & 0.9961 & 0.9959 & 0.9958 \\
\hline \multirow[t]{2}{*}{2492} & 0.9924 & 0.9937 & 0.9914 & 0.9913 & 0.9914 & 0.9962 & 0.9960 & 0.9959 \\
\hline & $\begin{array}{c}\mathrm{p}=1.8218 \\
\mathrm{GeV} / \mathrm{c}\end{array}$ & & & & & & & \\
\hline $249 \overline{6}$ & 0.9914 & 0.9928 & 0.9903 & 0.9900 & 0.9904 & 0.9958 & 0.9957 & 0.9956 \\
\hline 2497 & 0.9912 & 0.9930 & 0.9903 & 0.9900 & 0.9904 & 0.9959 & 0.9955 & 0.9956 \\
\hline 2498 & 0.9914 & 0.9930 & 0.9902 & 0.9901 & 0.9903 & 0.9958 & 0.9956 & 0.9956 \\
\hline 2499 & 0.9914 & 0.9929 & 0.9901 & 0.9900 & 0.9903 & 0.9956 & 0.9955 & 0.9955 \\
\hline 2500 & 0.9913 & 0.9928 & 0.9903 & 0.9899 & 0.9904 & 0.9958 & 0.9956 & 0.9956 \\
\hline 2501 & 0.9913 & 0.9929 & 0.9903 & 0.9899 & 0.9902 & 0.9958 & 0.9955 & 0.9955 \\
\hline 2502 & 0.9914 & 0.9929 & 0.9903 & 0.9900 & 0.9902 & 0.9957 & 0.9956 & 0.9955 \\
\hline 2503 & & & & & & & & \\
\hline 2504 & 0.9912 & 0.9930 & 0.9901 & 0.9901 & 0.9902 & 0.9957 & 0.9955 & 0.9956 \\
\hline 2505 & 0.9915 & 0.9929 & 0.9901 & 0.9901 & 0.9901 & 0.9957 & 0.9957 & 0.9955 \\
\hline 2506 & 0.9914 & 0.9930 & 0.9905 & 0.9902 & 0.9904 & 0.9959 & 0.9955 & 0.9954 \\
\hline 2507 & 0.9913 & 0.9929 & 0.9900 & 0.9900 & 0.9903 & 0.9958 & 0.9957 & 0.9955 \\
\hline \multirow[t]{2}{*}{2508} & 0.9913 & 0.9927 & 0.9901 & 0.9901 & 0.9902 & 0.9958 & 0.9956 & 0.9955 \\
\hline & $p=1.9600$ & & & & & & & \\
\hline KIN_3 & $\mathrm{GeV} / \mathrm{c}$ & & & & & & & \\
\hline 2509 & 0.9914 & 0.9929 & 0.9902 & 0.9901 & 0.9902 & 0.9960 & 0.9958 & 0.9956 \\
\hline 2510 & 0.9913 & 0.9930 & 0.9903 & 0.9901 & 0.9901 & 0.9958 & 0.9956 & 0.9957 \\
\hline 2511 & 0.9911 & 0.9928 & 0.9902 & 0.9901 & 0.9900 & 0.9959 & 0.9957 & 0.9958 \\
\hline 2512 & 0.9913 & 0.9930 & 0.9903 & 0.9901 & 0.9900 & 0.9959 & 0.9958 & 0.9956 \\
\hline 2513 & 0.9913 & 0.9928 & 0.9901 & 0.9900 & 0.9900 & 0.9958 & 0.9956 & 0.9958 \\
\hline 2514 & 0.9914 & 0.9929 & 0.9903 & 0.9900 & 0.9900 & 0.9959 & 0.9957 & 0.9957 \\
\hline 2515 & 0.9913 & 0.9928 & 0.9902 & 0.9900 & 0.9900 & 0.9960 & 0.9956 & 0.9957 \\
\hline 2516 & 0.9912 & 0.9927 & 0.9900 & 0.9900 & 0.9897 & 0.9959 & 0.9956 & 0.9955 \\
\hline 2517 & 0.9914 & 0.9929 & 0.9901 & 0.9902 & 0.9899 & 0.9957 & 0.9958 & 0.9957 \\
\hline 2518 & 0.9913 & 0.9930 & 0.9904 & 0.9900 & 0.9900 & 0.9958 & 0.9957 & 0.9957 \\
\hline 2519 & 0.9912 & 0.9928 & 0.9902 & 0.9902 & 0.9900 & 0.9959 & 0.9956 & 0.9957 \\
\hline 2520 & 0.9913 & 0.9930 & 0.9903 & 0.9903 & 0.9899 & 0.9959 & 0.9959 & 0.9956 \\
\hline 2521 & 0.9915 & 0.9930 & 0.9903 & 0.9903 & 0.9900 & 0.9959 & 0.9958 & 0.9957 \\
\hline
\end{tabular}

Table 64: VDC firing plane efficiencies for different plane combinations in the left(L) or right (R) arms (see Section 3.5). 


\begin{tabular}{lllllllllll}
\hline RUN & \# Events & RU1 & RV1 & RU2 & RV2 & LU1 & LV1 & LU2 & LV2 \\
\hline
\end{tabular}

KIN_1 $\quad \mathrm{p}=1.8665 \mathrm{GeV} / \mathrm{c}$

$\begin{array}{lcllllllll}2485 & 1002110 & 943860 & 943609 & 949489 & 947896 & 972091 & 971617 & 969785 & 964094 \\ 2486 & 1000709 & 942500 & 942444 & 948262 & 947084 & 970948 & 970480 & 968635 & 962877 \\ 2487 & 1000762 & 942582 & 942096 & 947845 & 946393 & 970598 & 970420 & 968620 & 962890 \\ 2488 & 737738 & 697667 & 697380 & 701781 & 700484 & 717724 & 717493 & 716460 & 712977 \\ 2489 & 1003256 & 950607 & 950304 & 956165 & 954643 & 978059 & 977614 & 976084 & 971137 \\ 2490 & 1004127 & 950541 & 950171 & 955729 & 954673 & 978389 & 978183 & 976700 & 971947 \\ 2491 & 1002629 & 950597 & 949972 & 955798 & 954634 & 977875 & 977706 & 976310 & 971598 \\ 2492 & 1003334 & 950509 & 950340 & 956102 & 954602 & 977611 & 977472 & 976017 & 971416\end{array}$

KIN_2

$\begin{array}{lccccccccc}2496 & 969408 & 915223 & 915067 & 921763 & 920367 & 950558 & 950463 & 948809 & 943697 \\ 2497 & 1003161 & 946821 & 946749 & 953578 & 951650 & 983486 & 983131 & 981520 & 976083 \\ 2498 & 1004753 & 948125 & 948018 & 954980 & 953302 & 984983 & 984692 & 983069 & 977697 \\ 2499 & 1002517 & 946096 & 945727 & 953042 & 951280 & 982721 & 982506 & 980630 & 975390 \\ 2500 & 1004037 & 947467 & 947476 & 954196 & 952510 & 984238 & 984024 & 982216 & 976906 \\ 2501 & 891242 & 841502 & 841345 & 847566 & 846018 & 873776 & 873551 & 872218 & 867331 \\ 2502 & 968900 & 913759 & 913668 & 920545 & 918928 & 949384 & 949181 & 947503 & 942289 \\ 2503 & 46 & 0 & 0 & 0 & 0 & 0 & 0 & 0 & 0 \\ 2504 & 1003623 & 947373 & 946999 & 954297 & 952324 & 984192 & 983838 & 982221 & 976761 \\ 2505 & 1005176 & 949012 & 948544 & 955497 & 953961 & 985350 & 985157 & 983393 & 977783 \\ 2506 & 403281 & 380776 & 380761 & 383548 & 382861 & 395357 & 395297 & 394606 & 392413 \\ 2507 & 1001755 & 945240 & 944856 & 952149 & 950495 & 982005 & 981792 & 980199 & 974797 \\ 2508 & 1002766 & 946919 & 946424 & 953413 & 951651 & 983174 & 983026 & 981259 & 975790\end{array}$

KIN 3

\begin{tabular}{llllllllll}
2509 & 1003722 & 947145 & 946861 & 953719 & 951858 & 982270 & 982121 & 980185 & 974495 \\
2510 & 1003801 & 946955 & 946669 & 953739 & 951850 & 982123 & 981841 & 980043 & 974398 \\
2511 & 1002591 & 946031 & 945739 & 952894 & 950968 & 981538 & 981156 & 979371 & 973606 \\
2512 & 1003558 & 946872 & 946622 & 953545 & 951419 & 981988 & 981856 & 980016 & 974284 \\
2513 & 1004075 & 947392 & 947035 & 954046 & 952203 & 982618 & 982165 & 980366 & 974726 \\
2514 & 1003080 & 946460 & 946387 & 953337 & 951516 & 981696 & 981453 & 979518 & 973809 \\
2515 & 1003835 & 946828 & 946848 & 953408 & 951530 & 982325 & 982005 & 980354 & 974549 \\
2516 & 1003819 & 947321 & 947007 & 953809 & 952090 & 982364 & 982168 & 980508 & 974487 \\
2517 & 1002734 & 946140 & 945592 & 952751 & 951017 & 981338 & 981152 & 979070 & 973350 \\
2518 & 1002785 & 946189 & 946157 & 953289 & 951243 & 981420 & 981148 & 979240 & 973606 \\
2519 & 1003331 & 946740 & 946278 & 953197 & 951383 & 981889 & 981620 & 979942 & 974195 \\
2520 & 1002094 & 945609 & 945258 & 952042 & 950128 & 980475 & 980356 & 978460 & 972611 \\
2521 & 1002432 & 946103 & 945731 & 952879 & 951133 & 980993 & 980916 & 978993 & 973241 \\
\hline
\end{tabular}

Table 65: VDC single plane number of hits in the left (L) or right $(\mathrm{R})$ arms (see Section $3.5)$. 


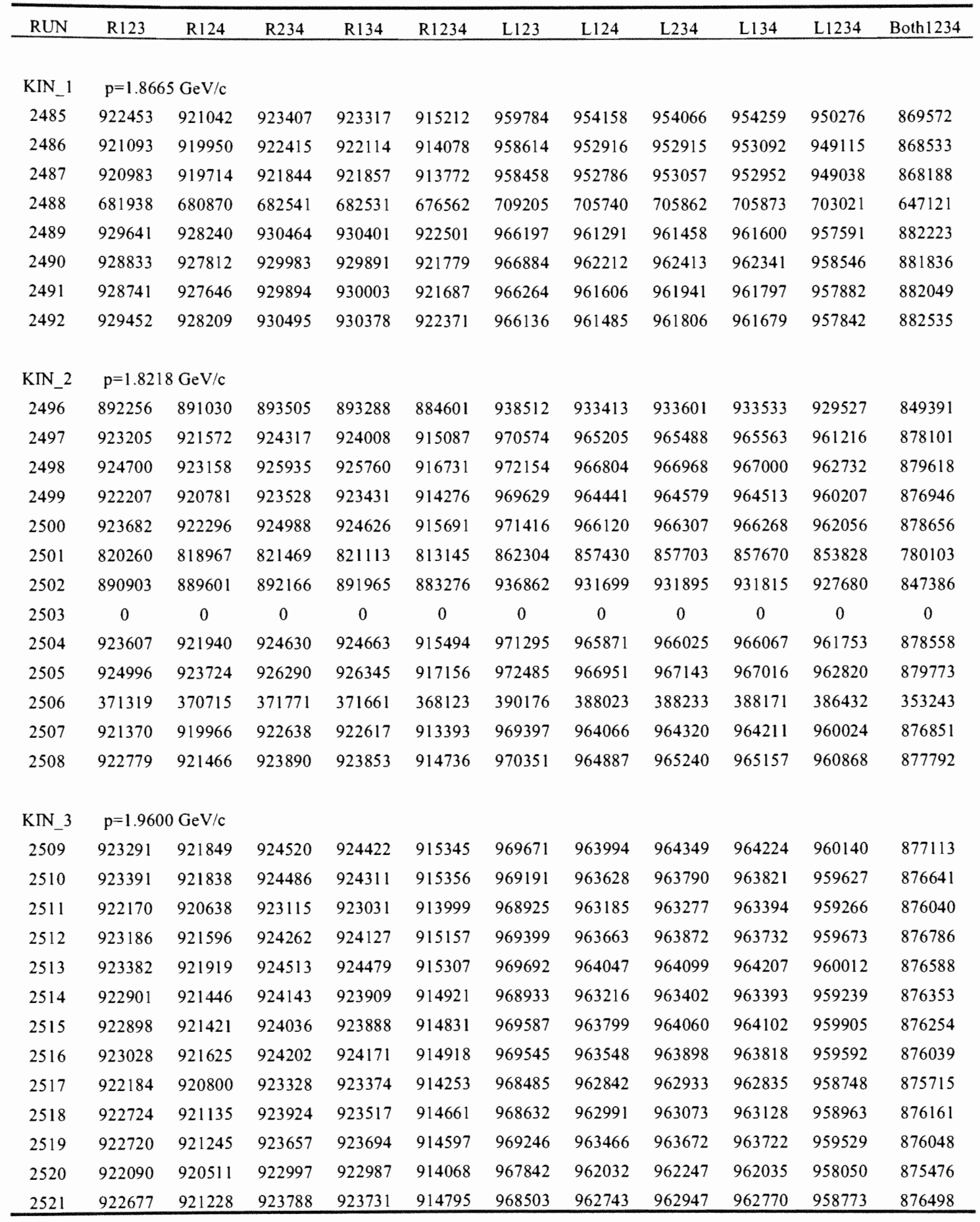

Table 66: VDC number of hits for each plane combination indicated in top row in the left (L) or right (R) arms (see Section 3.5). 


\begin{tabular}{|c|c|c|c|c|c|c|c|c|c|c|}
\hline RUN & $\begin{array}{l}\text { OTrack } \\
\mathrm{L}\end{array}$ & 1 TrackL & 2TrackL & 3TrackL & 4TrackL & $\begin{array}{l}\text { Good0 } \\
\text { TrackL } \\
\end{array}$ & $\begin{array}{l}\text { Goodl } \\
\text { TrackL } \\
\end{array}$ & $\begin{array}{l}\text { Good2 } \\
\text { TrackL } \\
\end{array}$ & $\begin{array}{l}\text { Good3 } \\
\text { TrackL } \\
\end{array}$ & $\begin{array}{l}\text { Good4 } \\
\text { TrackL }\end{array}$ \\
\hline $\mathrm{KIN}_{1}$ & \multicolumn{2}{|c|}{$\mathrm{p}=1.8665 \mathrm{GeV} / \mathrm{c}$} & & & & & & & & \\
\hline 2485 & 33883 & 901107 & 54843 & 11314 & 813 & 1662 & 886487 & 51483 & 10088 & 513 \\
\hline 2486 & 33375 & 898959 & 56013 & 11370 & 838 & 1512 & 884389 & 52512 & 10158 & 505 \\
\hline 2487 & 33675 & 899566 & 55389 & 11205 & 779 & 1583 & 885062 & 51869 & 10006 & 473 \\
\hline 2488 & 21654 & 666633 & 40529 & 8243 & 568 & 963 & 656297 & 38000 & 7360 & 370 \\
\hline 2489 & 27771 & 908742 & 55057 & 10757 & 785 & 1361 & 894395 & 51744 & 9558 & 488 \\
\hline 2490 & 27991 & 909380 & 54811 & 10951 & 814 & 1348 & 895386 & 51422 & 9826 & 511 \\
\hline 2491 & 26945 & 907752 & 55784 & 11211 & 779 & 1353 & 893524 & 52463 & 10021 & 473 \\
\hline 2492 & 27982 & 909067 & 54338 & 11068 & 723 & 1370 & 895040 & 50975 & 9965 & 446 \\
\hline \multicolumn{11}{|c|}{$\mathrm{KIN}_{-} \quad \mathrm{p}=1.8218 \mathrm{GeV} / \mathrm{c}$} \\
\hline 2496 & 21110 & 866910 & 65953 & 14091 & 1144 & 1467 & 852571 & 62090 & 12599 & 741 \\
\hline 2497 & 22006 & 896255 & 68636 & 14882 & 1138 & 1411 & 881425 & 64351 & 13230 & 731 \\
\hline 2498 & 22083 & 898055 & 68371 & 14859 & 1168 & 1395 & 883066 & 64256 & 13225 & 714 \\
\hline 2499 & 22322 & 895323 & 68827 & 14749 & 1087 & 1419 & 880228 & 64652 & 13171 & 663 \\
\hline 2500 & 22352 & 897054 & 68413 & 14808 & 1174 & 1500 & 882238 & 64218 & 13274 & 739 \\
\hline 2501 & 19416 & 795381 & 61898 & 13261 & 1085 & 1211 & 781965 & 58052 & 11849 & 692 \\
\hline 2502 & 21823 & 864721 & 66720 & 14295 & 1139 & 1387 & 850119 & 62637 & 12757 & 713 \\
\hline 2503 & 46 & 0 & 0 & 0 & 0 & 0 & 0 & 0 & 0 & 0 \\
\hline 2504 & 22140 & 896408 & 68878 & 14807 & 1183 & 1501 & 881463 & 64714 & 13223 & 780 \\
\hline 2505 & 22330 & 897673 & 69004 & 14760 & 1174 & 1427 & 882554 & 64793 & 13198 & 783 \\
\hline 2506 & 8825 & 360495 & 27413 & 5995 & 474 & 543 & 354455 & 25792 & 5318 & 296 \\
\hline 2507 & 22079 & 894549 & 68965 & 14822 & 1121 & 1485 & 879726 & 64763 & 13295 & 694 \\
\hline 2508 & 22039 & 895943 & 68595 & 14788 & 1191 & 1473 & 880961 & 64415 & 13192 & 753 \\
\hline \multicolumn{11}{|c|}{${ }_{3}^{\mathrm{KIN}_{-}} \quad \mathrm{p}=1.9600 \mathrm{GeV} / \mathrm{c}$} \\
\hline 2509 & 24356 & 902303 & 62737 & 13131 & 1010 & 1404 & 887278 & 58908 & 11817 & 665 \\
\hline 2510 & 24640 & 902821 & 62054 & 13116 & 964 & 1402 & 887724 & 58204 & 11629 & 613 \\
\hline 2511 & 24183 & 900853 & 63067 & 13292 & 1008 & 1406 & 886053 & 59240 & 11876 & 637 \\
\hline 2512 & 24594 & 902249 & 62282 & 13281 & 960 & 1472 & 887216 & 58435 & 11881 & 608 \\
\hline 2513 & 24425 & 902739 & 62741 & 12989 & 1000 & 1382 & 887465 & 58900 & 11574 & 635 \\
\hline 2514 & 24461 & 902351 & 61855 & 13238 & 991 & 1417 & 887286 & 58030 & 11824 & 626 \\
\hline 2515 & 24598 & 902400 & 62526 & 13113 & 1006 & 1408 & 887376 & 58695 & 11751 & 613 \\
\hline 2516 & 24322 & 901722 & 63124 & 13441 & 1004 & 1374 & 886366 & 59117 & 12060 & 620 \\
\hline 2517 & 24444 & 901271 & 62642 & 13189 & 1011 & 1385 & 885985 & 58873 & 11800 & 651 \\
\hline 2518 & 24409 & 901285 & 62764 & 13095 & 1026 & 1470 & 886060 & 58970 & 11729 & 677 \\
\hline 2519 & 24125 & 902063 & 62651 & 13316 & 954 & 1409 & 886857 & 58769 & 11851 & 583 \\
\hline 2520 & 24524 & 901780 & 61597 & 13088 & 933 & 1420 & 886500 & 57775 & 11705 & 584 \\
\hline 2521 & 24346 & 902220 & 61701 & 13045 & 961 & 1347 & 887273 & 57823 & 11680 & 601 \\
\hline
\end{tabular}

Table 67: Number of events with specific number of tracks in the the left arm (see Section 3.5). 


\begin{tabular}{|c|c|c|c|c|c|c|c|c|c|c|c|}
\hline RUN & $\begin{array}{l}0 \text { Trac } \\
\mathrm{kR}\end{array}$ & 1 TrackR & 2 TrackR & $\begin{array}{l}3 \text { Track } \\
\mathrm{R} \\
\end{array}$ & $\begin{array}{l}\text { 4Track } \\
\mathrm{R} \\
\end{array}$ & $\begin{array}{l}\text { Good0 } \\
\text { Track } \\
\mathrm{R} \\
\end{array}$ & $\begin{array}{l}\text { Good1 } \\
\text { TrackR } \\
\end{array}$ & $\begin{array}{l}\text { Good2 } \\
\text { TrackR } \\
\end{array}$ & $\begin{array}{l}\text { Good3 } \\
\text { TrackR } \\
\end{array}$ & $\begin{array}{l}\text { Good4 } \\
\text { TrackR } \\
\end{array}$ & $\begin{array}{l}\text { Goodl } \\
\text { Track } \\
\end{array}$ \\
\hline \multicolumn{12}{|l|}{$\mathrm{KN}_{1}$} \\
\hline 2485 & 42694 & 777671 & 125442 & 41567 & 9869 & 5120 & 760610 & 113926 & 31607 & 3481 & 674702 \\
\hline 2486 & 42501 & 776021 & 125576 & 41689 & 9979 & 5311 & 758897 & 114038 & 31889 & 3509 & 672538 \\
\hline 2487 & 42725 & 775576 & 125951 & 41530 & 10054 & 5011 & 758365 & 114449 & 31870 & 3592 & 672564 \\
\hline 2488 & 28018 & 576503 & 91932 & 30476 & 7184 & 3179 & 563951 & 83520 & 23110 & 2500 & 503765 \\
\hline 2489 & 36309 & 786304 & 124636 & 41304 & 9850 & 4311 & 769307 & 113392 & 31556 & 3533 & 687568 \\
\hline 2490 & 37041 & 786854 & 124280 & 41150 & 9832 & 4155 & 769603 & 113005 & 31204 & 3379 & 688243 \\
\hline 2491 & 35426 & 783948 & 126432 & 41851 & 10007 & 4286 & 766659 & 114929 & 31793 & 3592 & 684600 \\
\hline 2492 & 36545 & 786438 & 124530 & 41225 & 9889 & 4288 & 769573 & 113269 & 31432 & 3432 & 688551 \\
\hline \multicolumn{12}{|l|}{$\frac{\mathrm{KIN}_{2}}{-}$} \\
\hline 2496 & 34455 & 727842 & 140798 & 48308 & 12117 & 5085 & 710138 & 127526 & 36815 & 4507 & 625814 \\
\hline 2497 & 35958 & 753236 & 145659 & 49656 & 12516 & 5307 & 734884 & 131973 & 37699 & 4663 & 647149 \\
\hline 2498 & 36085 & 755461 & 145125 & 49598 & 12281 & 5176 & 737120 & 131666 & 37669 & 4509 & 648981 \\
\hline 2499 & 35761 & 752819 & 145619 & 49637 & 12524 & 5264 & 734211 & 131741 & 37884 & 4603 & 645877 \\
\hline 2500 & 36064 & 753703 & 145751 & 50086 & 12380 & 5217 & 735208 & 132205 & 37958 & 4578 & 647183 \\
\hline 2501 & 31216 & 667216 & 131246 & 44979 & 11179 & 4654 & 650696 & 118932 & 34249 & 4080 & 572212 \\
\hline 2502 & 35107 & 727125 & 140882 & 48080 & 11786 & 5094 & 709138 & 127593 & 36531 & 4389 & 623961 \\
\hline 2503 & 46 & 0 & 0 & 0 & 0 & 0 & 0 & 0 & 0 & 0 & 0 \\
\hline 2504 & 35677 & 752466 & 146234 & 50469 & 12682 & 5260 & 733753 & 132799 & 38370 & 4761 & 645908 \\
\hline 2505 & 36256 & 755646 & 145237 & 49747 & 12284 & 5366 & 737314 & 131467 & 37944 & 4514 & 648607 \\
\hline 2506 & 14256 & 303174 & 58463 & 19882 & 4999 & 2135 & 295885 & 52863 & 15185 & 1821 & 260581 \\
\hline 2507 & 35849 & 752446 & 145364 & 49619 & 12359 & 5269 & 733750 & 131623 & 37673 & 4535 & 646132 \\
\hline 2508 & 35733 & 753454 & 145417 & 49852 & 12274 & 5325 & 734681 & 131743 & 37868 & 4509 & 646710 \\
\hline \multicolumn{12}{|l|}{$\mathrm{KIN}_{2}$} \\
\hline 2509 & 36512 & 753489 & 145882 & 49583 & 12296 & 5307 & 735009 & 132236 & 37733 & 4530 & 651411 \\
\hline 2510 & 36750 & 753880 & 144913 & 50056 & 12169 & 5323 & 735587 & 131347 & 38094 & 4447 & 652189 \\
\hline 2511 & 35834 & 751233 & 146946 & 50277 & 12238 & 5334 & 732496 & 133075 & 38146 & 4425 & 648632 \\
\hline 2512 & 36614 & 753795 & 145229 & 49525 & 12329 & 5181 & 735419 & 131609 & 37796 & 4575 & 651461 \\
\hline 2513 & 36685 & 754568 & 144884 & 49462 & 12532 & 5317 & 735740 & 131343 & 37663 & 4686 & 651623 \\
\hline 2514 & 36397 & 753295 & 145300 & 49937 & 12078 & 5367 & 734890 & 131736 & 37959 & 4389 & 651465 \\
\hline 2515 & 36653 & 753547 & 145588 & 49523 & 12377 & 5309 & 734786 & 131909 & 37669 & 4589 & 651076 \\
\hline 2516 & 36059 & 750374 & 147875 & 50652 & 12612 & 5454 & 731743 & 133931 & 38436 & 4734 & 647648 \\
\hline 2517 & 36394 & 751879 & 145829 & 50138 & 12362 & 5381 & 733282 & 132214 & 38169 & 4643 & 649574 \\
\hline 2518 & 36013 & 751272 & 146699 & 50438 & 12234 & 5380 & 732787 & 133003 & 38393 & 4537 & 648835 \\
\hline 2519 & 36502 & 752269 & 146298 & 49742 & 12585 & 5367 & 733589 & 132535 & 37821 & 4728 & 649932 \\
\hline 2520 & 36760 & 753587 & 144492 & 49274 & 11936 & 5303 & 735274 & 131038 & 37423 & 4435 & 652029 \\
\hline 2521 & 36575 & 754524 & 144013 & 49333 & 12152 & 5355 & 736271 & 130700 & 37444 & 4490 & 653126 \\
\hline
\end{tabular}

Table 68: Number of events with specific number of tracks in the right arm (see Section $3.5)$. 
D CTOF spectra using aerogel detectors and RICH.

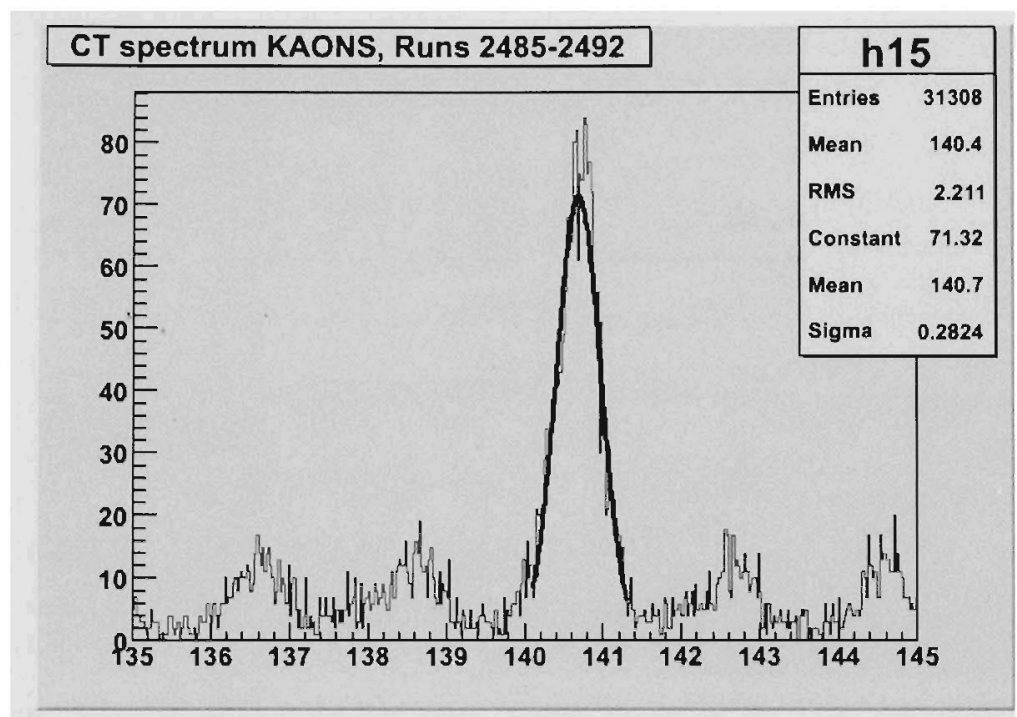

Figure 113: CT spectrum (ns) for particles identified as kaons (KIN_1).

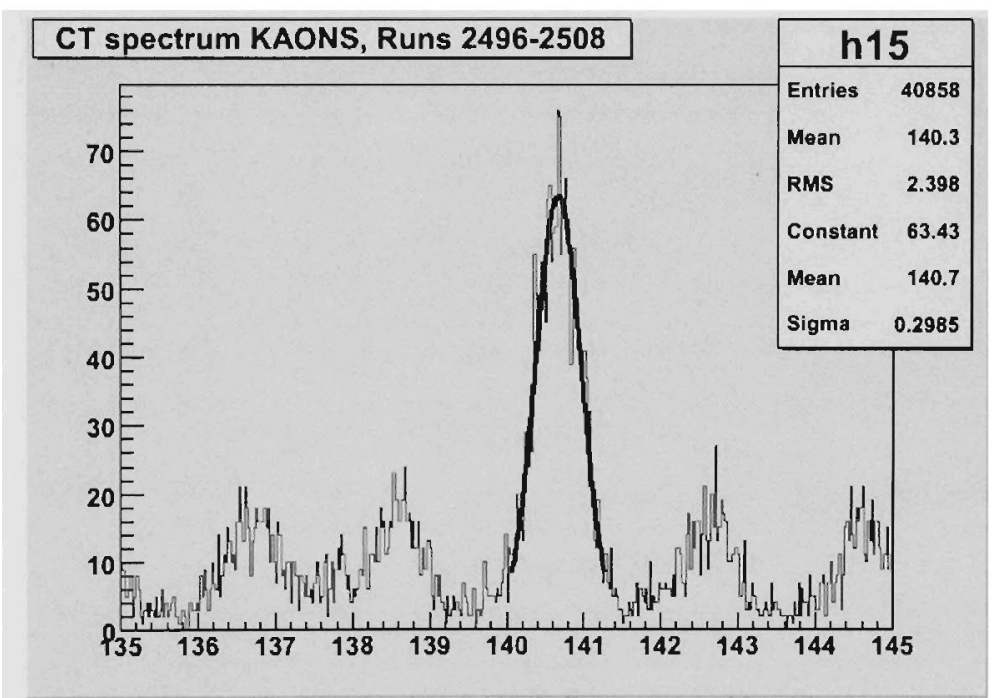

Figure 114: CT spectrum (ns) for particles identified as kaons (KIN_2). 


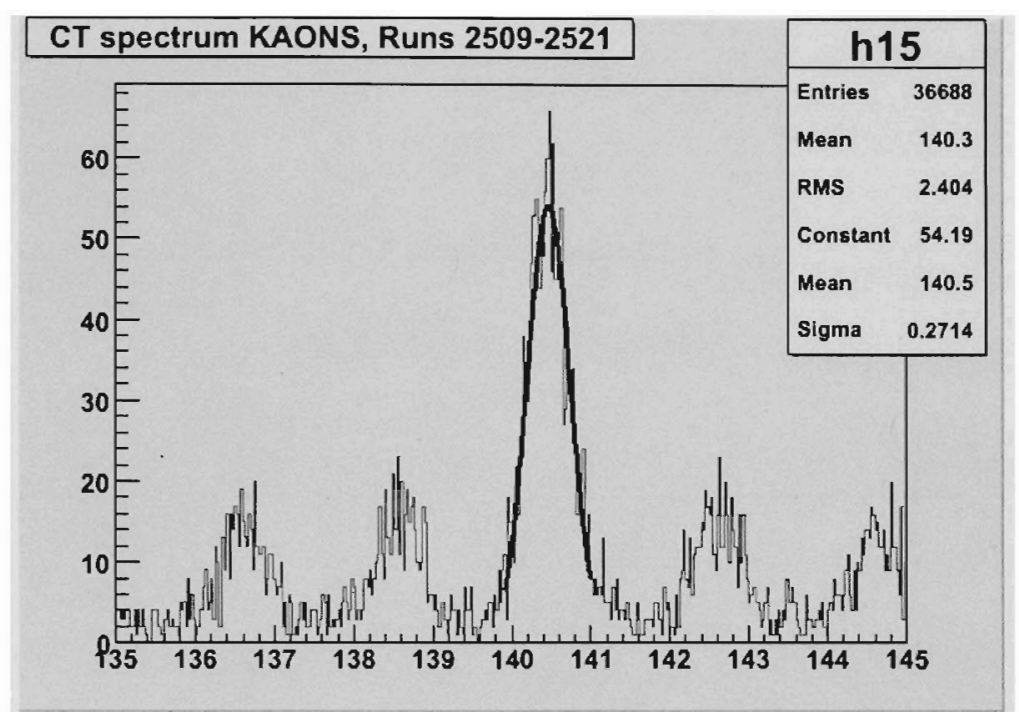

Figure 115: CT spectrum (ns) for particles identified as kaons (KIN_3).

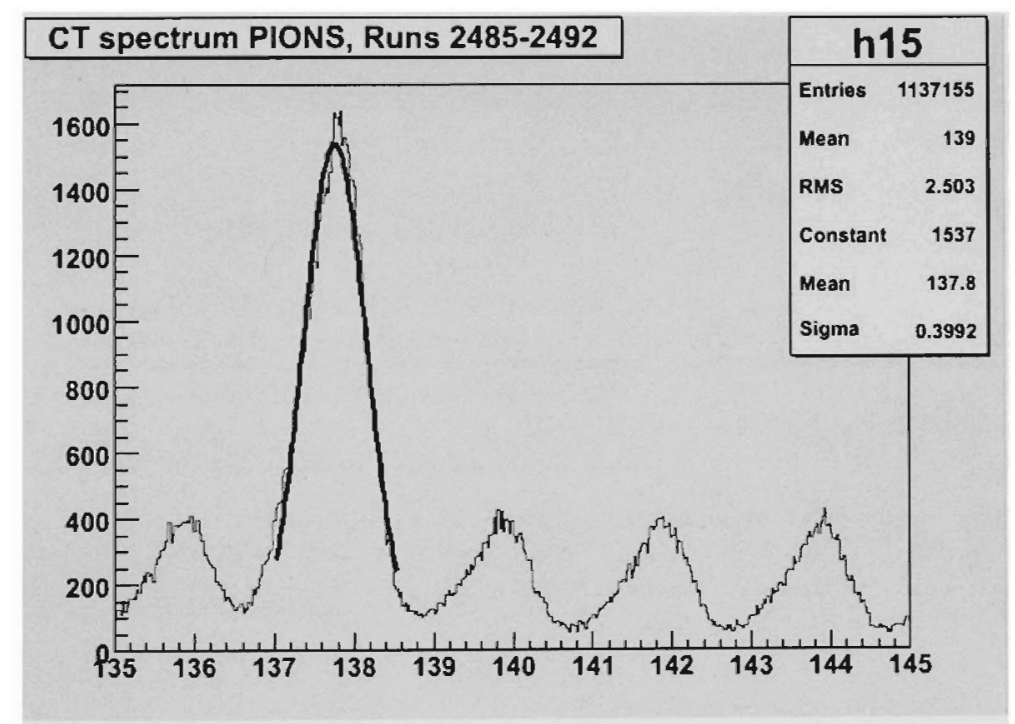

Figure 116: CT spectrum (ns) for particles identified as pions (KIN_1). 


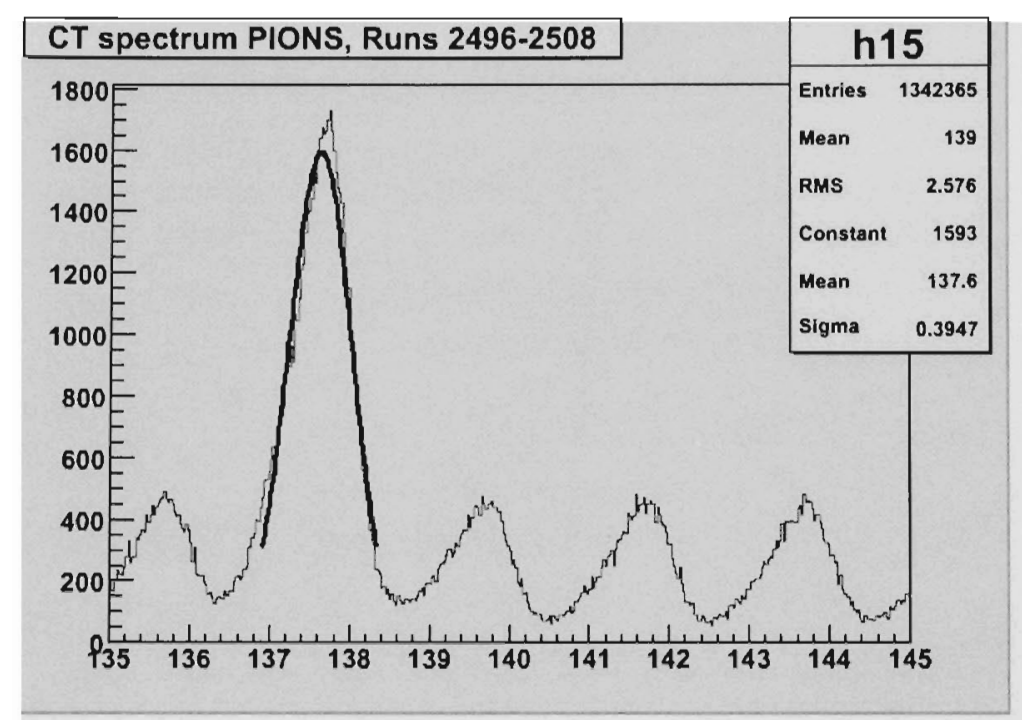

Figure 117: CT spectrum (ns) for particles identified as pions (KIN_2).

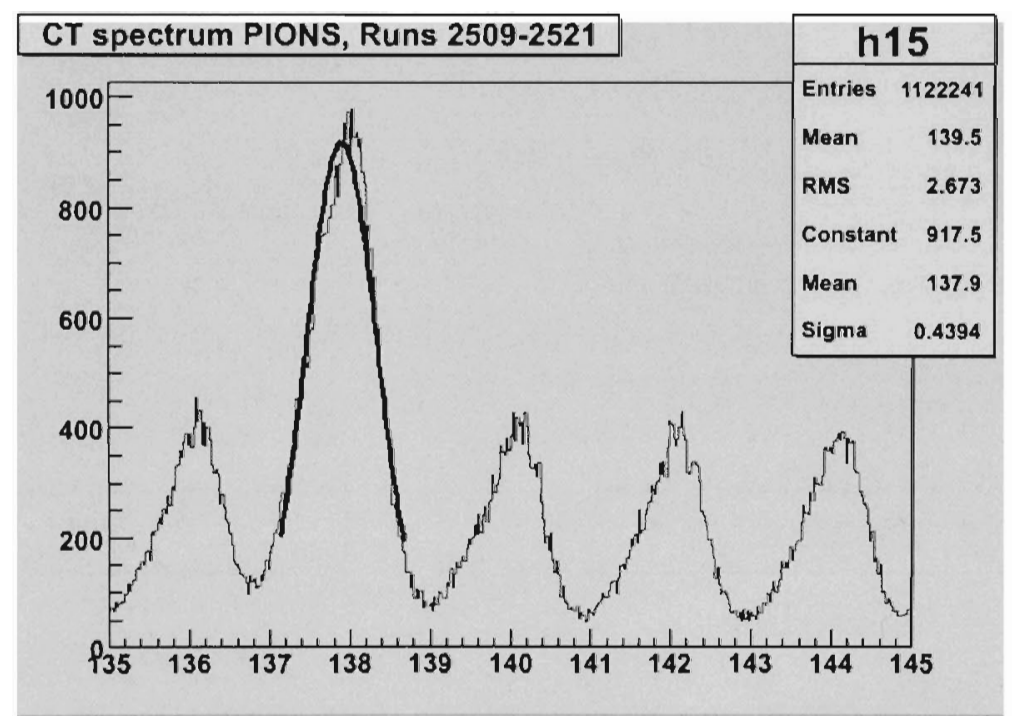

Figure 118: CT spectrum (ns) for particles identified as pions (KIN_3). 


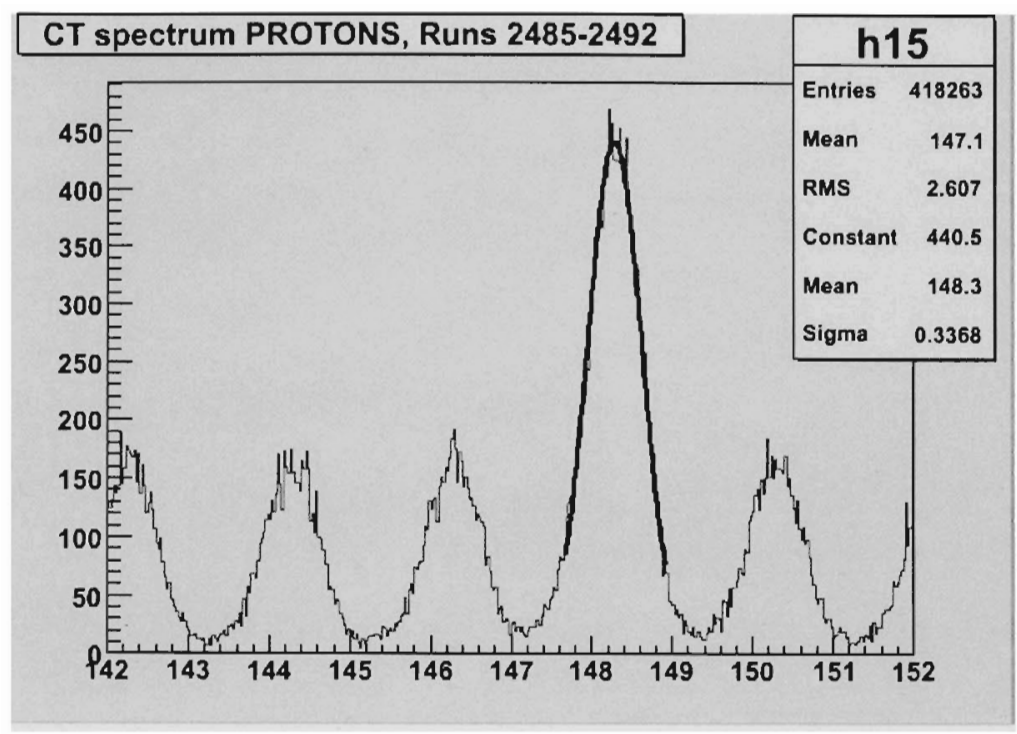

Figure 119: CT spectrum (ns) for particles identified as protons (KIN_1).

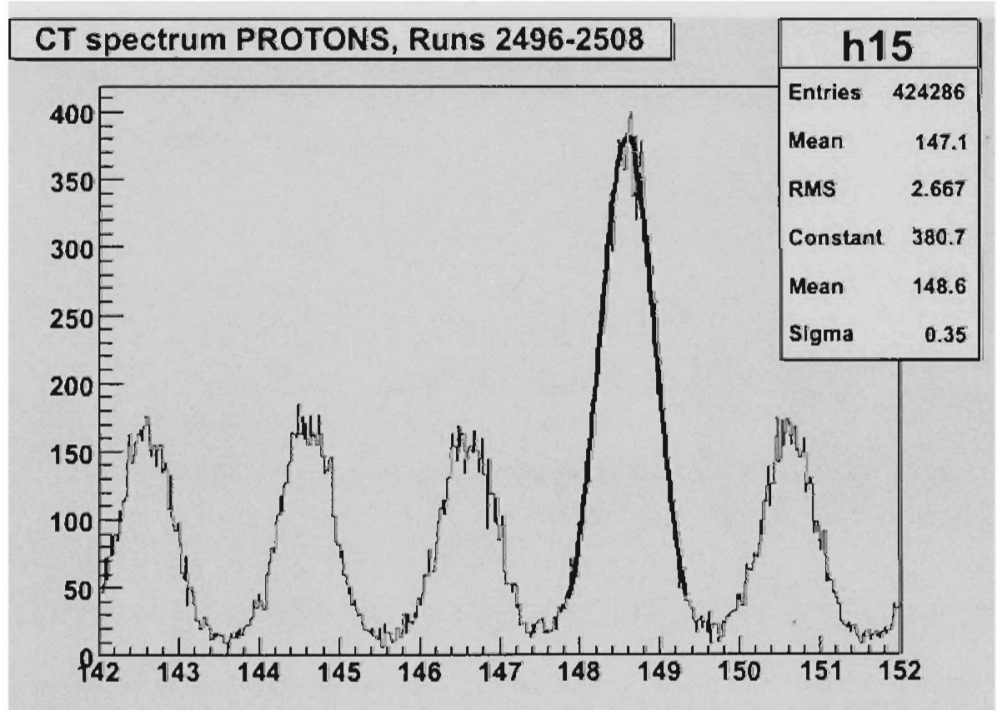

Figure 120: CT spectrum (ns) for particles identified as protons (KIN_2). 


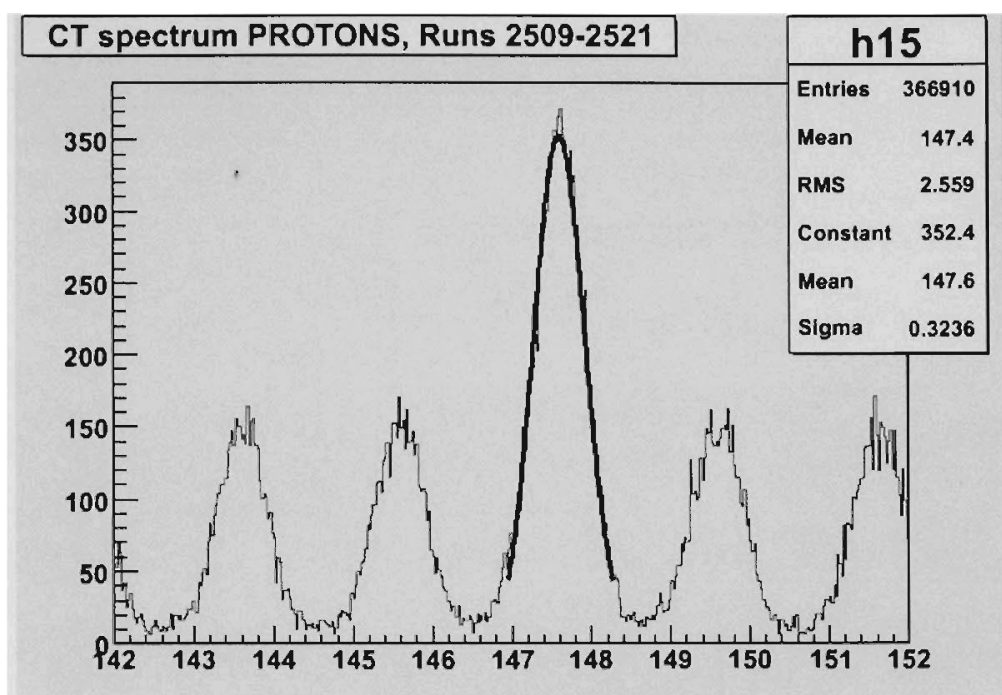

Figure 121: CT spectrum (ns) for particles identified as protons (KIN_3). 
E Missing mass spectra (see Section 4.2) with RICH PID cuts included (Table 17).

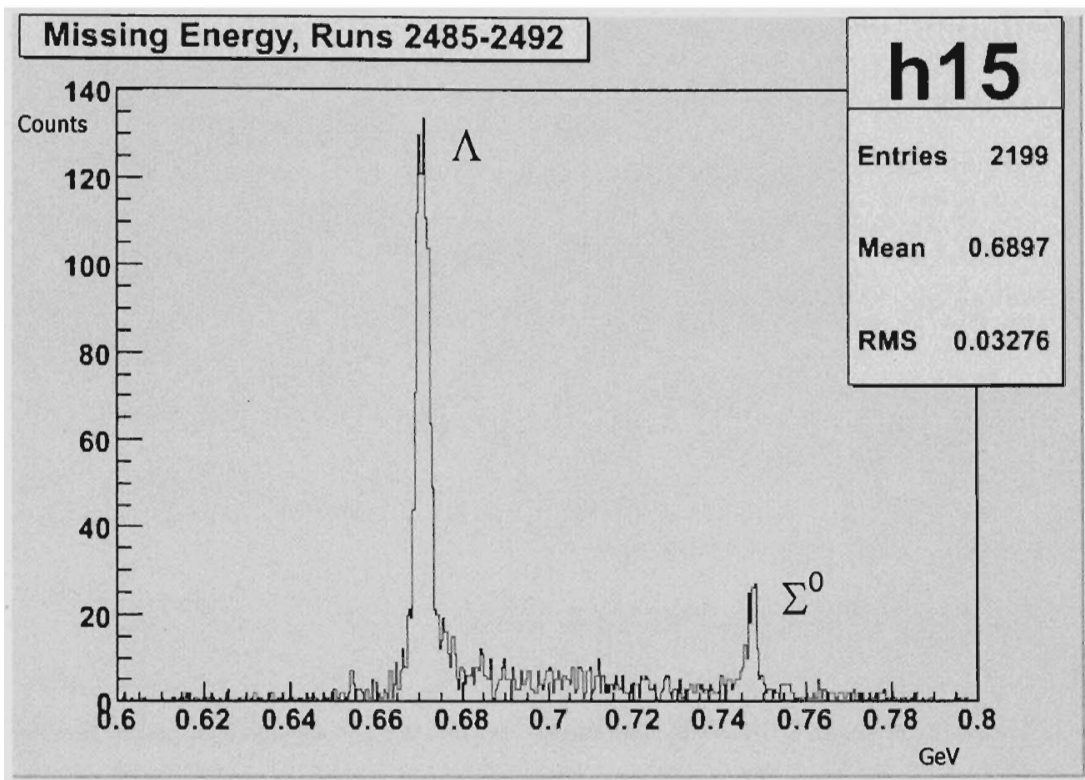

Figure 122: Missing mass spectrum with RICH PID cuts for KIN_1.

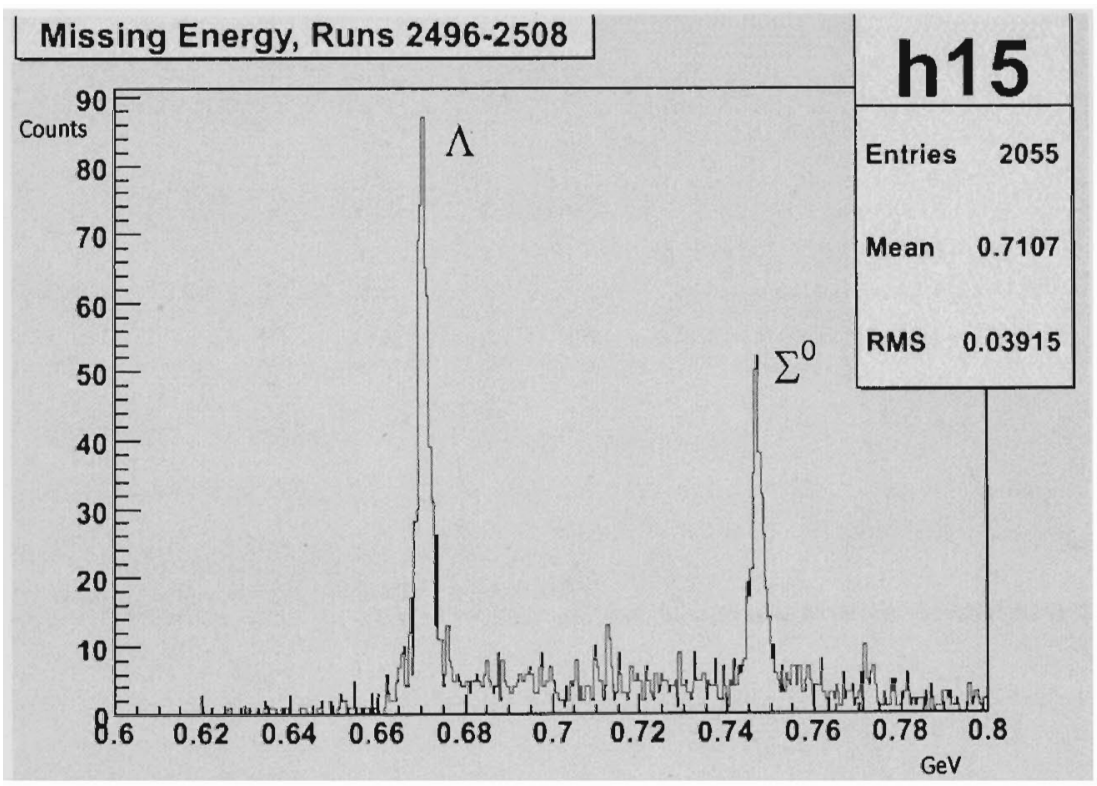

Figure 123: Missing mass spectrum with RICH PID cuts for KIN_2. 


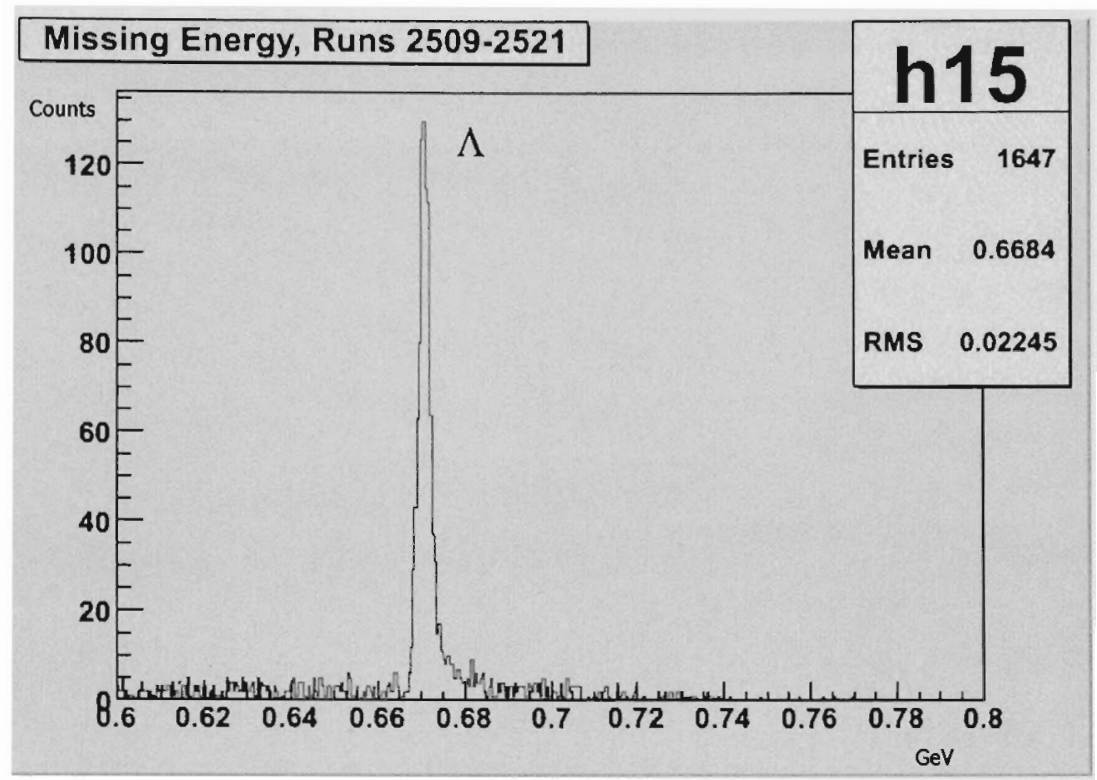

Figure 124: Missing mass spectrum with RICH PID cuts for KIN_3. 
F Subtracted missing mass spectra (see Section 4.3) with RICH PID cuts (Table 17).

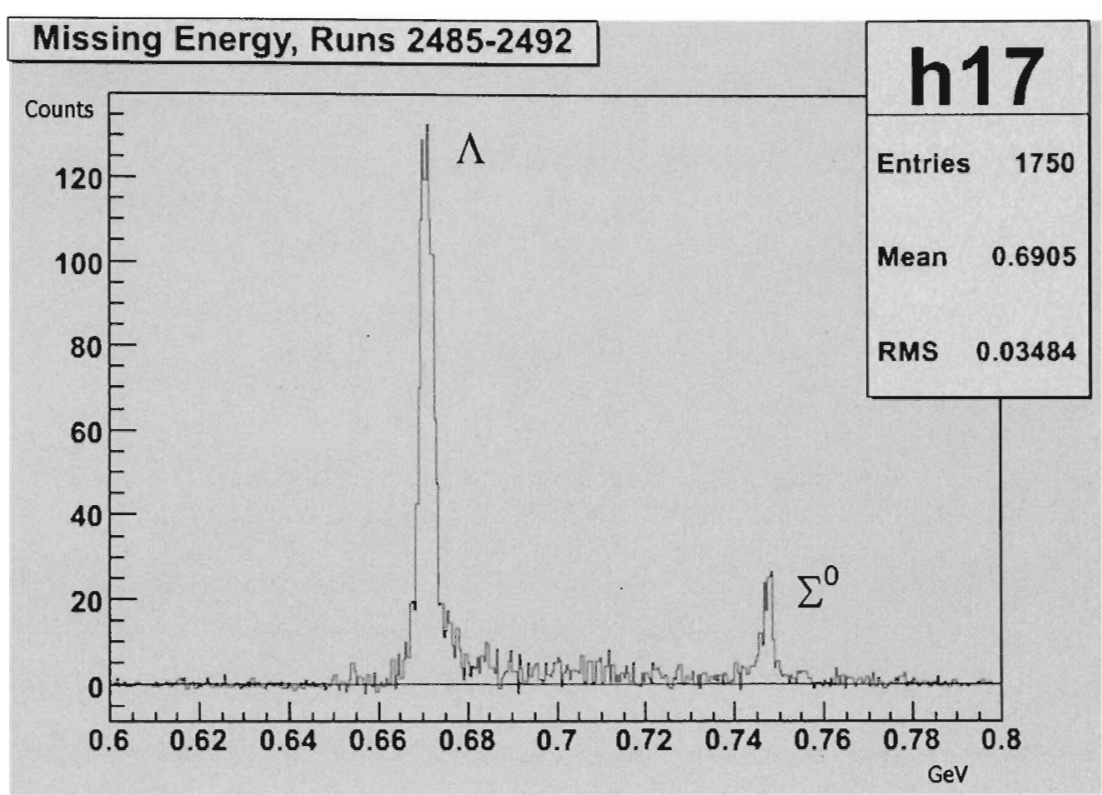

Figure 125: Background substracted spectrum with RICH PID cuts for KIN_1.

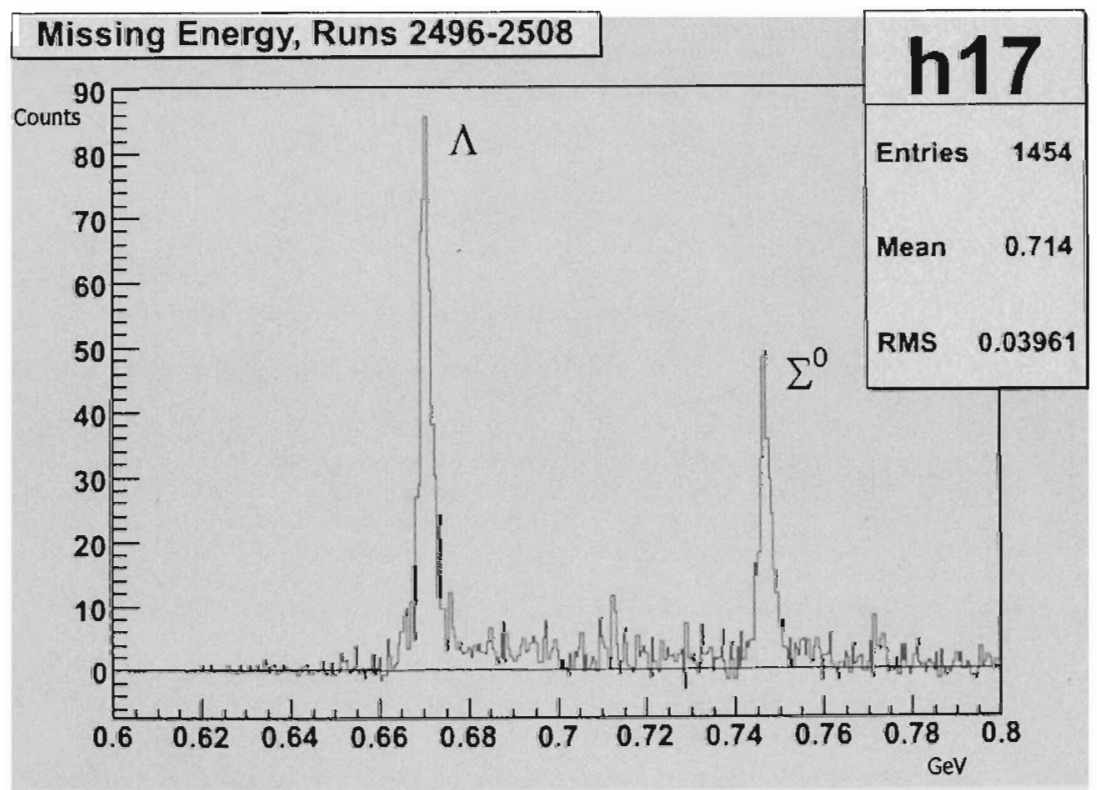

Figure 126: Background substracted spectrum with RICH PID cuts for KIN_2. 


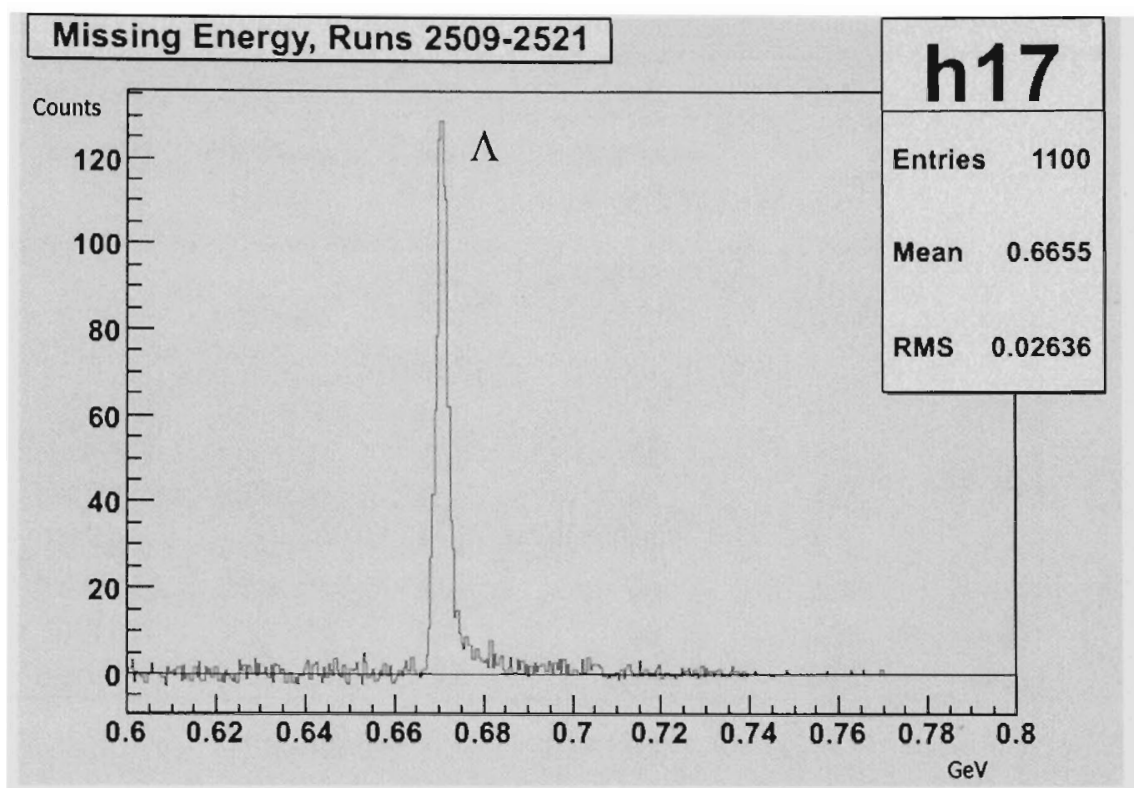

Figure 127: Background substracted spectrum with RICH PID cuts for KIN_3. 
G RICH efficiencies for the three different kinematics.

\begin{tabular}{|c|c|c|c|c|c|c|c|}
\hline KAONS & Skeb.emiss & AERO: & 857 & 347 & 822 & & \\
\hline Ro min & Ro $\max$ & $\mathrm{R}$ mean & Delta R & $\mathrm{R}$ interval & & & \\
\hline 0.621 & 0.66 & 0.6405 & 0.039 & 0.004875 & & & \\
\hline \multirow[t]{2}{*}{ \#interval } & $\mathrm{R} \min$ & $\mathrm{R} \max$ & $\begin{array}{l}\text { AERO } \\
+\mathrm{RICH}\end{array}$ & $\begin{array}{l}\text { ACCIDENTAL } \\
\left({ }^{*} 10\right)\end{array}$ & TRUE & Normalized & Uncertainty \\
\hline & & & TOTAL & & & & \\
\hline-3 & 0.635625 & 0.645375 & 318 & 35 & 314 & 0.3820 & 0.0169 \\
\hline-2 & 0.63075 & 0.65025 & 470 & 55 & 464 & 0.5645 & 0.0173 \\
\hline-1 & 0.625875 & 0.655125 & 513 & 64 & 506 & 0.6156 & 0.0170 \\
\hline 0 & 0.621 & 0.66 & 518 & 65 & 511 & 0.6217 & 0.0169 \\
\hline 1 & 0.616125 & 0.664875 & 520 & 66 & 513 & 0.6241 & 0.0169 \\
\hline 2 & 0.61125 & 0.66975 & 522 & 69 & 515 & 0.6265 & 0.0169 \\
\hline 3 & 0.606375 & 0.674625 & 522 & 72 & 514 & 0.6253 & 0.0169 \\
\hline
\end{tabular}

Table 69: RICH efficiency for KIN_1.

RICH EFFICIENCY FOR RUN 2485-2492 WITH AEROGEL

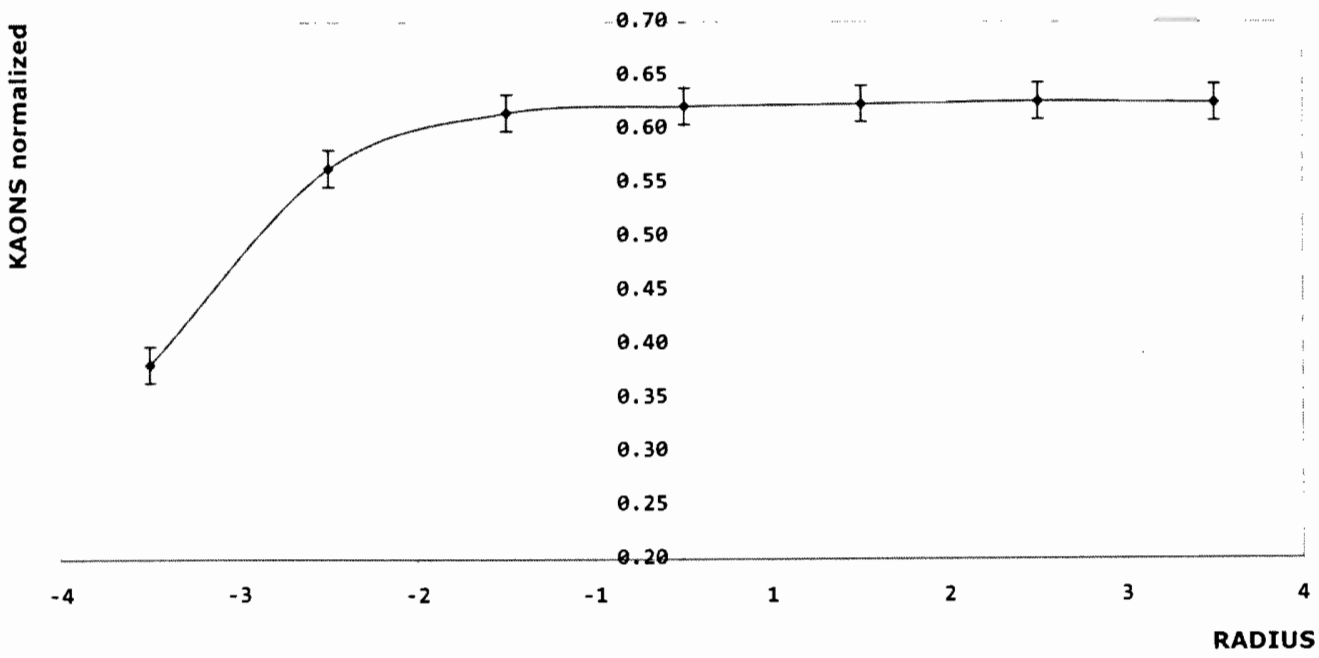

Figure 128: RICH efficiency for KIN_1. 


\begin{tabular}{cccccccc}
\hline KAONS & Skeb.emiss & AERO: & 394 & 206 & 373 & \\
\hline Ro min & Ro max & R mean & Delta R & R interval & & & \\
0.621 & 0.66 & 0.6405 & 0.039 & 0.004875 & & & \\
& & & & & & \\
\#interval & R min & R max & AERO & ACCIDENTAL & & & \\
& & & TOTAL & & & & \\
-3 & 0.635625 & 0.645375 & 190 & 34 & 186 & 0.4987 & 0.0259 \\
-2 & 0.63075 & 0.65025 & 257 & 46 & 252 & 0.6756 & 0.0242 \\
-1 & 0.625875 & 0.655125 & 272 & 50 & 267 & 0.7158 & 0.0234 \\
0 & 0.621 & 0.66 & 275 & 50 & 270 & 0.7239 & 0.0231 \\
1 & 0.616125 & 0.664875 & 275 & 51 & 269 & 0.7212 & 0.0232 \\
2 & 0.61125 & 0.66975 & 275 & 52 & 269 & 0.7212 & 0.0232 \\
3 & 0.606375 & 0.674625 & 277 & 54 & 271 & 0.7265 & 0.0231 \\
\hline
\end{tabular}

Table 70: RICH efficiency for KIN_2.

RICH EFFICIENCY FOR RUN 2496-2508 WITH AEROGEL

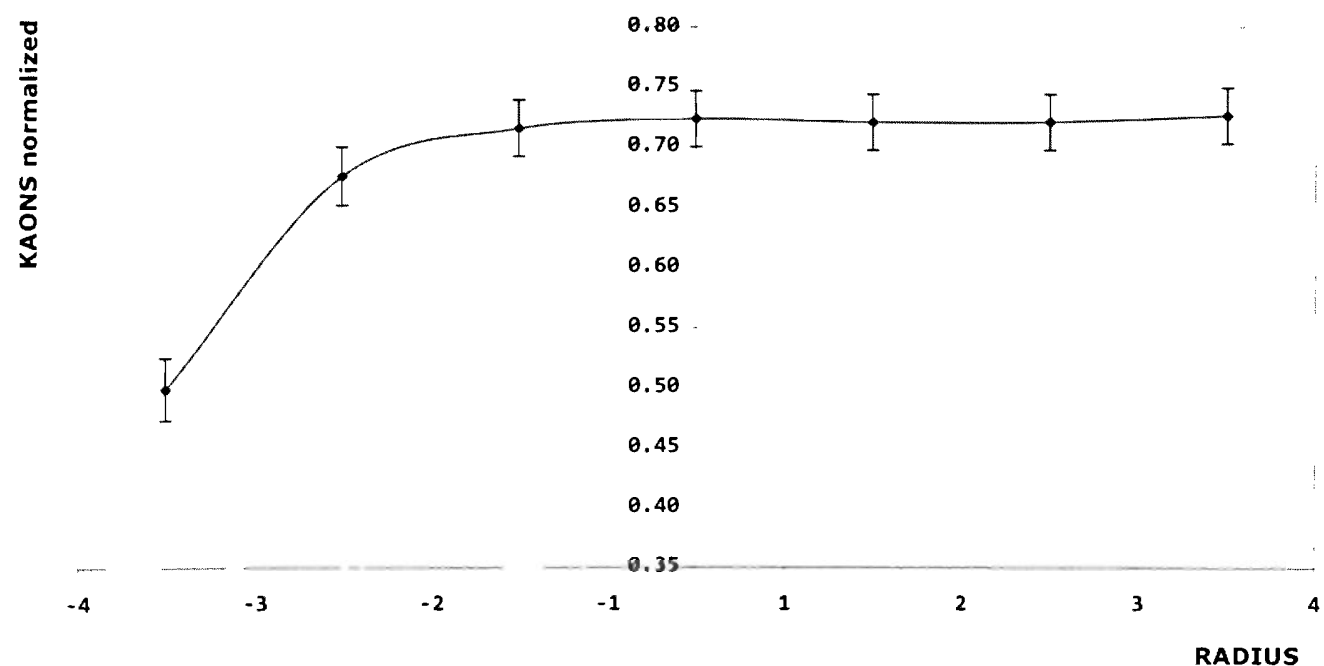

Figure 129: RICH efficiency for KIN_2. 


\begin{tabular}{cccccccc}
\hline KAONS & Skeb.emiss & AERO: & 816 & 326 & 783 & \\
\hline Ro min & Ro max & R mean & Delta R & R interval & & & \\
0.621 & 0.66 & 0.6405 & 0.039 & 0.004875 & & & \\
& & & & & & \\
\#interval & R min & R max & AERO & ACCIDENTAL & & & \\
& & & TOTAL & $\left({ }^{*} 10\right)$ & TRUE & Normalized & Uncertainty \\
-3 & 0.635625 & 0.645375 & 199 & 34 & 195 & 0.2490 & 0.0155 \\
-2 & 0.63075 & 0.65025 & 322 & 44 & 317 & 0.4049 & 0.0175 \\
-1 & 0.625875 & 0.655125 & 356 & 54 & 350 & 0.4470 & 0.0178 \\
0 & 0.621 & 0.66 & 362 & 55 & 356 & 0.4547 & 0.0178 \\
1 & 0.616125 & 0.664875 & 363 & 55 & 357 & 0.4559 & 0.0178 \\
2 & 0.61125 & 0.66975 & 364 & 56 & 358 & 0.4572 & 0.0178 \\
3 & 0.606375 & 0.674625 & 364 & 57 & 358 & 0.4572 & 0.0178 \\
\hline
\end{tabular}

Table 71: RICH efficiency for KIN_3.

RICH EFFICIENCY FOR RUN 2509-2521 WITH AEROGEL

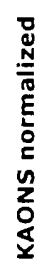

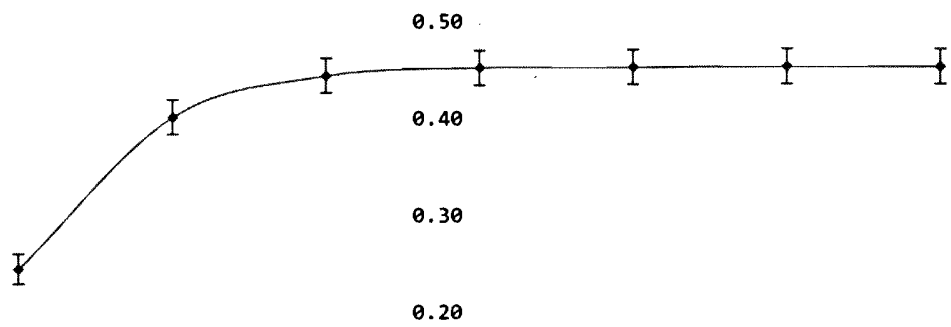

0.10

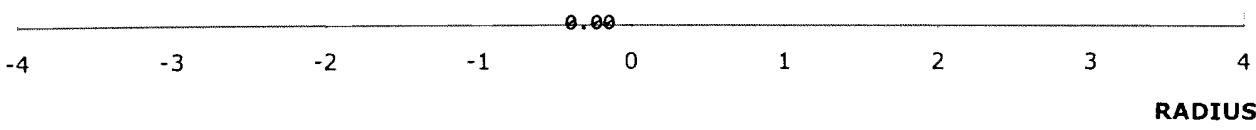

Figure 130: RICH efficiency for KIN_3. 
program kfit

c empirical fit to electroproduction data by Jinseok Cha, similar to Brauel

c on linux compile with: f77 -o kfit3 kfit3.f

c LAST REVISED: 28-Mar-08, changed inputs to $\mathrm{pk}$ and thetacm

implicit none

real*8 pi, degrad, alpha, me, mp, mk, mlam, mk2, mp2, $\mathrm{mL} 2$

parameter $(p i=3.141592653589$, degrad=pi $/ 180$. , al pha=1./137.0359895)

parameter $(\mathrm{me}=0.000511, \mathrm{mp}=0.938272, \mathrm{mk}=0.493646, \mathrm{mlam}=1.192642)$

parameter $(m k 2=m k * m k, m p 2=m p * m p, m L 2=m L a m * m L a m)$

real*8 Ebeam, $W, Q Q, t$

! initial conditions

real*8 omega, qvec, tha, epsi

! virtual photon

real*8 Ek, pk, thk, thgk, betak, gammak, survive ! kaon

real*8 $\mathrm{Ee}, \mathrm{pe}$, thedeg, the, $q \mathrm{~cm}$, omcm

! scattered electron

real*8 bcm, gcm, s, pcm, tmin, tmax

real*8 pkx,pkz,pkxcm,pkzcm, thgkcm,pkcm,gtpr, qdotk

real*8 phi, cosphi

real*8 dum1, dum2, dum3, dum4, dum5, dum6, dum7

integer iq2, iread, iang

real*8 Sigma_eep, jac, rate, Lumin, accept, time, Sigma

c *** kinematic input -- $E_{-} 0(\mathrm{GeV}), \mathrm{Q} 2(\mathrm{GeV} / \mathrm{C})^{\wedge} 2, \mathrm{~W}(\mathrm{GeV}), \mathrm{t}(\mathrm{GeV} / \mathrm{c})^{\wedge} 2$ write $\left(*,{ }^{*}\right)$ 'beam energy $(\mathrm{GeV})$ '

$\operatorname{read}(*, *) \quad$ Ebeam

write $(*, *)$ ' $Q 2+(\mathrm{GeV} / \mathrm{c})^{\wedge} 2$ and $W(\mathrm{GeV}) '$

$\operatorname{read}(*, *) \quad Q Q, W$

write $(*, *)$ 'Pk $(\mathrm{GeV} / \mathrm{C})$, Kaon final momentum'

$\operatorname{read}(*, *) \quad p k$

write $\left(*,{ }^{*}\right)$ 'th qk lab (deg),'

$\operatorname{read}(*, *)$ thgk

thgk $=$ thgk $^{*}$ degrad

C

*** scattered electron

$\mathrm{Ee}=$ Ebeam $-\left(((W * W)+Q Q-m p 2) /\left(2{ }^{*} m p\right)\right)$

the $=2 .{ }^{*} \operatorname{asin}\left(\operatorname{sqrt}\left(\mathrm{QQ} /\left(4 .{ }^{*}\right.\right.\right.$ Ebeam*Ee $\left.\left.)\right)\right)$

thedeg=the/degrad

pe $=\operatorname{sqrt}\left(E e^{*} E e-m^{*} m e\right)$

C

*** virtual photon ***

omega = Ebeam-Ee

qvec $=\operatorname{sqrt}(\mathrm{QQ}+$ omega*omega $)$

tha $=\operatorname{atan}\left(E e^{*} \sin (\right.$ the $) /\left(E\right.$ beam $\left.\left.-\left(E e^{*} \cos (t h e)\right)\right)\right)$

qvec $=\operatorname{sqrt}(Q Q+$ omega*omega $)$

epsi $=1 . /\left(1 .+2 .{ }^{*}(1 .+\right.$ omega**2. $/ Q Q) * \tan ($ the $\left./ 2) * * 2.\right)$

C

*** photon-proton center-of-mass system $* * *$

$\mathrm{bcm}=q v e c /$ (omega $+\mathrm{mp})$

$\mathrm{gcm}=1 . / \operatorname{sqrt}(1 .-\mathrm{bcm} * \mathrm{bcm})$

$\mathrm{s}=\mathrm{W} W$ 


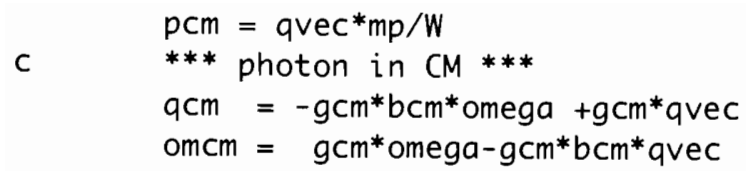

C $\quad * * *$ kaon $* * *$

tmin $=((-Q Q-m k 2-m p 2+m L 2) /(2.0 * W)) * * 2.0$

\# $\quad-(\operatorname{sart}(((W * W-Q Q-m p 2) /(2 . * W)) * * 2 .+Q Q)$

\# $\left.\quad-\operatorname{sgrt}\left(\left((W * W+m k 2-m L 2) /\left(2 .{ }^{*} W\right)\right)^{* * 2},-m k 2\right)\right) * * 2$.

tmax $=((-Q Q-m k 2-m p 2+m L 2) /(2.0 * W)) * * 2.0$

\# $\quad-\left(\operatorname{sqrt}\left(\left(W^{*} W-Q Q-m p 2\right) /(2 . * W)\right) * * 2 .+Q Q\right)$

$\left.\left.\# \quad+\operatorname{sqrt}\left(\left(W^{*} W+m k 2-m L 2\right) /(2 . * W)\right) * * 2 .-m k 2\right)\right) * * 2$.

C $\quad * * *$ kaon with respect to photon

$E k=\operatorname{sqrt}\left(\mathrm{pk}^{*} \mathrm{pk}+\mathrm{mk}^{*} \mathrm{mk}\right)$

qdotk $=$ qvec*pk* $\cos ($ thgk);

$t=-Q Q+m k 2-\left(2^{*} E k^{*}\right.$ omega $)+\left(2^{*}\right.$ qdotk $)$;

$c / * t=($ gamma $-k) 2 * /$

if $(t$. eq. $\theta)$ then

$t=t \min -1.0 \mathrm{E}-9$

endif

cosphi $=-1$

if (cosphi.eq.1) then

thk $=-$ (thq-thgk)

elseif (cosphi.eq. -1) then

thk $=-($ thq + thgk $)$

endif

$p k x=p k^{*} \sin ($ thgk)

$p k z=p k^{*} \cos ($ thgk $)$

$p k x c m=p k x$

$p k z c m=-g c m * b c m * E k+g c m * p k z$

$p k c m=\operatorname{sqrt}\left(p k x c m^{*} p k x c m+p k z c m^{*} p k z c m\right)$

thgkcm $=\operatorname{atan}(p k x c m / p k z c m)$

jac $=p k^{*} p k^{*} W / p k c m / a b s\left((m p+o m e g a) * p k-E k^{*} q v e c^{*} \cos (\right.$ thgk $\left.)\right)$

c phi=3.1415

if (cosphi.eq.1) then

phi $=0$.

elseif(cosphi.eq.-1) then phi $=p i$

endif

call eekeek(QQ,W, tmin, phi, epsi, sigma,t,pkcm)

sigma $=\operatorname{sigma*}(2 .+$ epsi $) / 3$.

!INCLUDE EPSILON INTO D

SIGMA

*** Virtual photon flux

gtpr $=a$ lpha $/ 2 . /\left(p^{* *} 2\right) *$ Ee/Ebeam*(s-mp**2)/2./mp/QQ

\# $/(1$, -epsi)

sigma_eep=sigma*gtpr*jac

!d5 sigma/dE'd0mega_e 


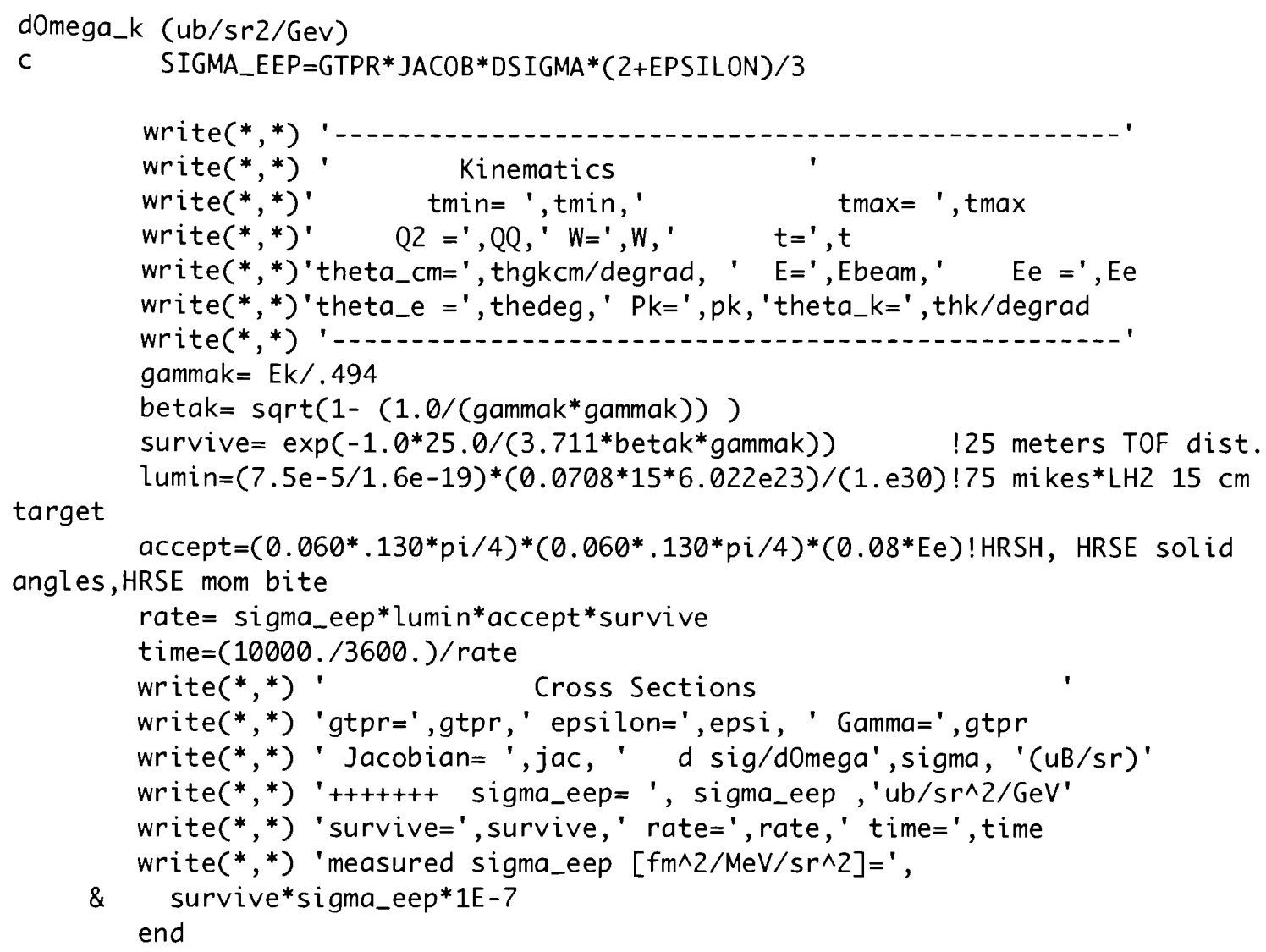




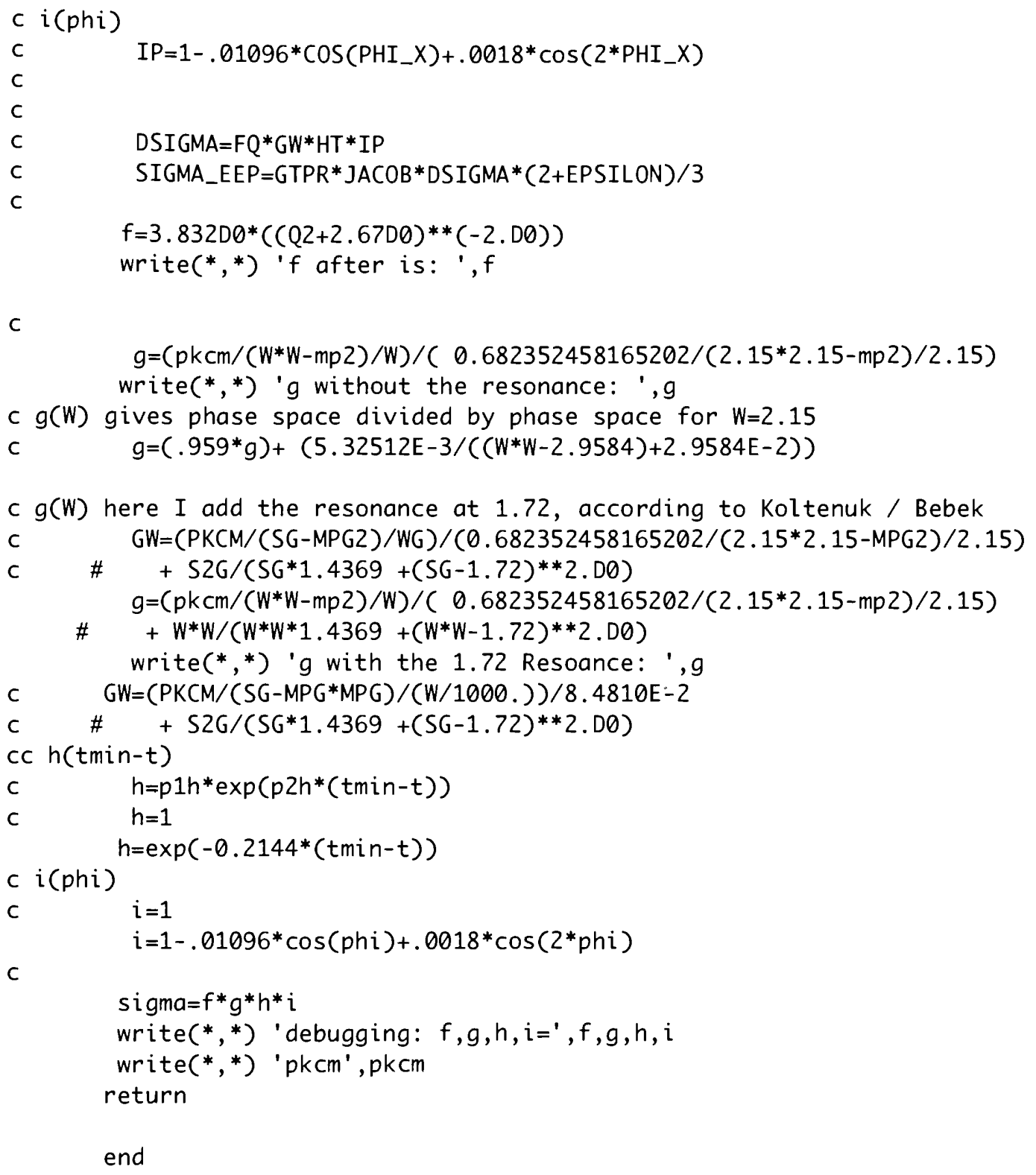


I MCEEP summary outputs (see Section 1.3). In addition to variable definitions shown in page 138, some extra variables are defined here as [72]:

Phi_Q $=\phi_{q}$, the horizontal angle of $\vec{q}$ with respect to the "laboratory" coordinate system,

Theta_Q $=\theta_{q}$, the vertical angle of $\vec{q}$ with respect to the "laboratory" coordinate system,

P_recoil $=p_{r}$, the momentum of the recoiling nuclear system, Theta $\_\mathrm{pq}=\theta_{p q}$, the angle between the emerging hadron and $\vec{q}$.

\begin{tabular}{ccccccc}
\hline Variable & Central & Average & Centroid & Minimum & Maximum & Units \\
\hline Scatt.Angle $\left(\theta_{e}\right)$ & 6 & 6.383 & 6.25 & 5.084 & 8.1 & $\mathrm{deg}$ \\
Omega $\left(E-E^{\prime}\right)$ & 2201.9 & 2184.218 & 2183.286 & 2137.1 & 2262.951 & $\mathrm{MeV}$ \\
Q-vector $(|\vec{q}|)$ & 2216.636 & 2201.364 & 2199.751 & 2148.715 & 2284.471 & $\mathrm{MeV} / \mathrm{c}$ \\
Q_mu ${ }^{2}\left(Q^{2}\right)$ & 0.065 & 0.075 & 0.072 & 0.045 & 0.123 & $(\mathrm{GeV} / \mathrm{c})^{2}$ \\
Phi_Q $\left(\phi_{q}\right)$ & 4.256 & 4.5 & 4.412 & 3.392 & 5.809 & $\mathrm{deg}$ \\
Theta_Q $\left(\theta_{q}\right)$ & 0 & 0.02 & 0.017 & -1.958 & 1.941 & $\mathrm{deg}$ \\
X (Bjorken_x $)$ & 0.016 & 0.018 & 0.018 & 0.011 & 0.03 & \\
Long. Pol. $(\varepsilon)$ & 0.707 & 0.712 & 0.712 & 0.685 & 0.728 & \\
P_f $\left(p_{k}\right)$ & 1866.5 & 1893.401 & 1894.794 & 1793.095 & 1942.697 & $\mathrm{MeV} / \mathrm{c}$ \\
P_recoil $\left(p_{r}\right)$ & 355.565 & 319.597 & 317.134 & 288.912 & 401.737 & $\mathrm{MeV} / \mathrm{c}$ \\
Theta_pq $\left(\theta_{p q}\right)$ & 1.744 & 2.242 & 2.295 & 0.004 & 5.12 & $\mathrm{deg}$ \\
Phi_x $(\varphi)$ & 180 & 178.885 & 178.924 & 0.316 & 359.355 & $\mathrm{deg}$ \\
M_miss $\left(E_{m i s s}\right)$ & 711.449 & 676.428 & 674.767 & 665.478 & 711.088 & $\mathrm{MeV}$ \\
W $(W)$ & 2.224 & 2.214 & 2.215 & 2.187 & 2.253 & $\mathrm{GeV}$ \\
Theta_CM $\left(\theta_{C M}\right)$ & 4.574 & 5.85 & 5.984 & 0.012 & 13.314 & $\mathrm{deg}$ \\
\hline
\end{tabular}

Table 72: MCEEP output summary for L_KIN_1. 


\begin{tabular}{ccccccc}
\hline Variable & Central & Average & Centroid & Minimum & Maximum & Units \\
\hline Scatt.Angle $\left(\theta_{e}\right)$ & 6 & 6.399 & 6.269 & 5.143 & 7.961 & $\mathrm{deg}$ \\
Omega $\left(E-E^{\prime}\right)$ & 2206.8 & 2166.055 & 2164.294 & 2141.997 & 2234.68 & $\mathrm{MeV}$ \\
Q-vector $(|\vec{q}|)$ & 2221.522 & 2183.692 & 2181.264 & 2153.982 & 2251.989 & $\mathrm{MeV} / \mathrm{c}$ \\
Q_mu ${ }^{2}\left(Q^{2}\right)$ & 0.065 & 0.077 & 0.074 & 0.048 & 0.118 & $(\mathrm{GeV} / \mathrm{c})^{2}$ \\
Phi_Q $\left(\phi_{q}\right)$ & 4.247 & 4.613 & 4.53 & 3.547 & 5.798 & $\mathrm{deg}$ \\
Theta_Q $\left(\theta_{q}\right)$ & 0 & 0.008 & 0.007 & -1.944 & 1.933 & $\mathrm{deg}$ \\
X (Bjorken_$)$ & 0.016 & 0.019 & 0.018 & 0.011 & 0.029 & \\
Long.Pol. $(\varepsilon)$ & 0.706 & 0.718 & 0.719 & 0.697 & 0.727 & \\
P_f $\left(p_{k}\right)$ & 1821.8 & 1872.116 & 1873.267 & 1798.927 & 1896.159 & $\mathrm{MeV} / \mathrm{c}$ \\
P_recoil $\left(p_{r}\right)$ & 404.433 & 322.69 & 319.605 & 291.693 & 399.243 & $\mathrm{MeV} / \mathrm{c}$ \\
Theta_pq $\left(\theta_{p q}\right)$ & 1.753 & 2.223 & 2.273 & 0.013 & 5.092 & $\mathrm{deg}$ \\
Phi_x $(\varphi)$ & 180 & 179.818 & 179.828 & 0.455 & 358.641 & $\mathrm{deg}$ \\
M_miss $\left(E_{\text {miss }}\right)$ & 746.166 & 678.067 & 675.97 & 665.993 & 711.086 & $\mathrm{MeV}$ \\
W $(W)$ & 2.226 & 2.206 & 2.206 & 2.189 & 2.24 & $\mathrm{GeV}$ \\
Theta_CM $\left(\theta_{C M}\right)$ & 4.624 & 5.789 & 5.912 & 0.033 & 13.191 & $\mathrm{deg}$ \\
\hline
\end{tabular}

Table 73: MCEEP output summary for L_KIN_2.

\begin{tabular}{ccccccc}
\hline Variable & Central & Average & Centroid & Minimum & Maximum & Units \\
\hline Scatt.Angle $\left(\theta_{e}\right)$ & 6 & 6.384 & 6.254 & 5.084 & 8.052 & $\mathrm{deg}$ \\
Omega $\left(E-E^{\prime}\right)$ & 2205.84 & 2218.397 & 2218.332 & 2160.721 & 2266.817 & $\mathrm{MeV}$ \\
Q-vector $(|\vec{q}|)$ & 2220.513 & 2234.926 & 2234.205 & 2172.898 & 2289.335 & $\mathrm{MeV} / \mathrm{c}$ \\
Q_mu ${ }^{2}\left(Q^{2}\right)$ & 0.065 & 0.074 & 0.071 & 0.045 & 0.116 & $(\mathrm{GeV} / \mathrm{c})^{2}$ \\
Phi_Q $\left(\phi_{q}\right)$ & 4.238 & 4.337 & 4.251 & 3.365 & 5.634 & $\mathrm{deg}$ \\
Theta_Q $\left(\theta_{q}\right)$ & 0 & 0.036 & 0.032 & -1.899 & 1.891 & $\mathrm{deg}$ \\
X (Bjorken_x $)$ & 0.016 & 0.018 & 0.017 & 0.011 & 0.028 & \\
Long.Pol. $(\varepsilon)$ & 0.706 & 0.701 & 0.701 & 0.684 & 0.72 & \\
P_f $\left(p_{k}\right)$ & 1960 & 1931.92 & 1933.421 & 1882.035 & 1993.133 & $\mathrm{MeV} / \mathrm{c}$ \\
P_recoil $\left(p_{r}\right)$ & 268.293 & 315.932 & 314.303 & 287.012 & 402.521 & $\mathrm{MeV} / \mathrm{c}$ \\
Theta_pq $\left(\theta_{p q}\right)$ & 1.762 & 2.33 & 2.385 & 0.01 & 5.201 & $\mathrm{deg}$ \\
Phi_x $(\varphi)$ & 180 & 177.98 & 178.151 & 0.807 & 358.808 & $\mathrm{deg}$ \\
M_miss $\left(E_{m i s s}\right)$ & 645.778 & 674.245 & 673.129 & 663.403 & 711.086 & $\mathrm{MeV}$ \\
W $(W)$ & 2.226 & 2.229 & 2.23 & 2.199 & 2.256 & $\mathrm{GeV}$ \\
Theta_CM $\left(\theta_{C M}\right)$ & 4.588 & 6.102 & 6.242 & 0.027 & 13.579 & $\mathrm{deg}$ \\
\hline
\end{tabular}

Table 74: MCEEP output summary for L_KIN_3. 


\begin{tabular}{cccccccc}
\hline Variable & Central & Aver & age & Centroid & Minimum & Maximum & Units \\
\hline Scatt.Angle $\left(\theta_{e}\right)$ & 6 & 6 & 380 & 6.251 & 5.084 & 8.046 & $\mathrm{deg}$ \\
Omega $\left(E-E^{\prime}\right)$ & 2201.9 & 2223 & 782 & 2223.151 & 2181.544 & 2263.018 & $\mathrm{MeV}$ \\
Q-vector $(|\vec{q}|)$ & 2216.636 & 2240 & 191 & 2238.919 & 2194.112 & 2285.487 & $\mathrm{MeV} / \mathrm{c}$ \\
Q_mu $\left(Q^{2}\right)$ & 0.065 & 0 & 73 & 0.07 & 0.045 & 0.117 & $(\mathrm{GeV} / \mathrm{c})^{2}$ \\
Phi_Q $\left(\phi_{q}\right)$ & 4.256 & 4 & 309 & 4.226 & 3.382 & 5.536 & $\mathrm{deg}$ \\
Theta_Q $\left(\theta_{q}\right)$ & 0 & 0 & 32 & 0.029 & -1.853 & 1.856 & $\mathrm{deg}$ \\
X (Bjorken_$)$ & 0.016 & 0 & 18 & 0.017 & 0.011 & 0.028 & \\
Long. Pol. $(\varepsilon)$ & 0.707 & 0 & 699 & 0.7 & 0.685 & 0.714 & \\
P_f $\left(p_{k}\right)$ & 1866.5 & 1829 & 292 & 1830.312 & 1792.225 & 1870.92 & $\mathrm{MeV} / \mathrm{c}$ \\
P_recoil $\left(p_{r}\right)$ & 355.565 & 420 & 120 & 418.251 & 393.478 & 502.623 & $\mathrm{MeV} / \mathrm{c}$ \\
Theta_pq $\left(\theta_{p q}\right)$ & 1.744 & 2 & 334 & 2.391 & 0.02 & 5.346 & $\mathrm{deg}$ \\
Phi_x $(\varphi)$ & 180 & 178 & 69 & 178.254 & 0.657 & 359.334 & $\mathrm{deg}$ \\
M_miss $\left(E_{\text {miss }}\right)$ & 711.449 & 751 & 7 & 749.954 & 741.363 & 788.011 & $\mathrm{MeV}$ \\
W $(W)$ & 2.224 & 2 & 232 & 2.232 & 2.206 & 2.254 & $\mathrm{GeV}$ \\
Theta_CM $\left(\theta_{C M}\right)$ & 4.574 & 6 & 178 & 6.325 & 0.052 & 14.13 & $\mathrm{deg}$ \\
\hline
\end{tabular}

Table 75: MCEEP output summary for S_KIN_1.

\begin{tabular}{ccccccc}
\hline Variable & Central & Average & Centroid & Minimum & Maximum & Units \\
\hline Scatt.Angle $\left(\theta_{e}\right)$ & 6 & 6.396 & 6.271 & 5.141 & 8.087 & $\mathrm{deg}$ \\
Omega $\left(E-E^{\prime}\right)$ & 2206.8 & 2208.21 & 2208.473 & 2141.995 & 2267.936 & $\mathrm{MeV}$ \\
Q-vector $(|\vec{q}|)$ & 2221.522 & 2225.054 & 2224.666 & 2153.687 & 2290.744 & $\mathrm{MeV} / \mathrm{c}$ \\
Q_mu $\left(Q^{2}\right)$ & 0.065 & 0.075 & 0.072 & 0.046 & 0.12 & $(\mathrm{GeV} / \mathrm{c})^{2}$ \\
Phi_Q $\left(\phi_{q}\right)$ & 4.247 & 4.409 & 4.323 & 3.411 & 5.762 & $\mathrm{deg}$ \\
Theta_Q $\left(\theta_{q}\right)$ & 0 & 0.028 & 0.024 & -1.944 & 1.935 & $\mathrm{deg}$ \\
X (Bjorken_x $)$ & 0.016 & 0.018 & 0.017 & 0.011 & 0.029 & \\
Long. Pol. $(\varepsilon)$ & 0.706 & 0.705 & 0.705 & 0.684 & 0.727 & \\
P_f $\left(p_{k}\right)$ & 1821.8 & 1812.211 & 1814.328 & 1749.396 & 1886.249 & $\mathrm{MeV} / \mathrm{c}$ \\
P_recoil $\left(p_{r}\right)$ & 404.433 & 421.678 & 419.558 & 393.671 & 502.013 & $\mathrm{MeV} / \mathrm{c}$ \\
Theta_pq $\left(\theta_{p q}\right)$ & 1.753 & 2.304 & 2.357 & 0.004 & 5.265 & $\mathrm{deg}$ \\
Phi_x $(\varphi)$ & 180 & 178.194 & 178.361 & 0.917 & 359.525 & $\mathrm{deg}$ \\
M_miss $\left(E_{\text {miss }}\right)$ & 746.166 & 751.405 & 750.269 & 741.646 & 788.038 & $\mathrm{MeV}$ \\
W $(W)$ & 2.226 & 2.225 & 2.225 & 2.189 & 2.256 & $\mathrm{GeV}$ \\
Theta_CM $\left(\theta_{C M}\right)$ & 4.624 & 6.091 & 6.226 & 0.011 & 13.925 & $\mathrm{deg}$ \\
\hline
\end{tabular}

Table 76: MCEEP output summary for S_KIN_2. 


\section{VITA}

\section{ARMANDO R. ACHA}

2009- Cyberknife radiosurgery system. Medical physicist at Mercy Hospital, Miami FL.

2004-2011 Doctoral candidate, Florida International University.

2001-2003 M.Sc. (Physics), Portland State University, Portland, Oregon.

1988-1996 B.Sc. (Physics), "Universidad Nacional de Ingeniería”, National University of Engineering, Lima, Peru.

2005- $\quad$ Member of American Physical Society (APS).

2005-2009 Member of Thomas Jefferson National Accelerator Facility User Group.

1992-2001 Member of the Theoretical Physics Research Group, "Universidad Nacional de Ingeniería", Professional School of Physics, National University of Engineering, Lima, Peru.

1994

Rescue of a long unused program (designed by NOAA) to calculate a radio link between Jicamarca and the peruvian base in Antarctica in the bandwidth of 2-30 MHz, Jicamarca's radio observatory "Instituto Geofísico del Perú”, Geophysical Institute of Peru, Lima, Peru.

\section{PUBLICATIONS}

1. Low $Q^{2}$ kaon electroproduction. P. Markowitz and A. Acha. Int.J.Mod.Phys. E19 (2010) 2383-2386.

2. Measurement of the electric form factor of the neutron up to $Q^{2}=3.4 \mathrm{GeV}^{2}$ using the reaction ${ }^{3} \mathrm{He}\left(\bar{e}, e^{\prime} n\right) p p$. S. Riodan, ...A. Acha et al., Phys.Rev.Lett. 105 (2010) 262302 .

3. High Resolution Spectroscopy of ${ }_{\Lambda}^{16} \mathrm{~N}$ by Electroproduction. F. Cusanno, ... A. Acha et al., Phys.Rev.Lett. 103 (2009) 202501.

4. Performance of the Two Aerogel Cherenkov Detectors of the JLab Hall A Hadron Spectrometer. S. Marrone, ... A. Acha et al., Nuovo Cim. B124 (2009) 99-114.

5. Kaon, pion and proton associated fission of Bi nuclei with electromagnetic probes $\mathrm{Y}$. Song,...A. Acha et al., Hall C, TJNAF. Phys. of Atom. Nucl. 73, 1707 (2010). 
6. Hypernuclear spectroscopy program at Jlab Hall C. O. Hashimoto, ... A. Acha et al., Nucl. Phys. A 804, 125-138 (2008).

7. Hypernuclear spectroscopy via $\left(e, e^{\prime} K^{+}\right)$in JLab's Hall A. J. Lerose, ... A. Acha et al., Nucl. Phys. A 804, 116-124 (2008).

8. High Resolution Spectroscopy of ${ }_{\Lambda}^{12}$ B by Electroproduction M. Iodice, ... A. Acha et al., Phys. Rev. Lett. 99, 052501 (2007).

9. Precision Measurements of the Nucleon Strange Form Factors at $Q^{2} \sim 0.1 \mathrm{GeV}^{2} \mathrm{~A}$. Acha et al., Phys. Rev. Lett. 98, 032301 (2007).

10. The HKS experiment on $\Lambda$-hypernuclear spectroscopy via electroproduction at Jlab L. Tang, ... A. Acha et al., Nucl. Phys. A 790, 679c-682c (2007).

\section{CONTRIBUTED TALKS AND POSTER PRESENTATIONS}

1. Kaon Electroproduction at low $Q^{2}$ and $W=2.2 \mathrm{GeV}$ off hydrogen. A. Acha, P. Markowitz, ... et al., contributed talk at the April meeting of the American Physical Society (APS), Denver, Colorado, 2-5 May 2009.

2. Electroproduction of Hyperons at Low Momentum Transfer. A. Acha, P. Markowitz, ... et al., contributed talk at the $75^{\text {th }}$ Annual Meeting of the

Southeastern Section of the APS (SESAPS), Raleigh, North Carolina, 30 Oct- 1 Nov 2008 .

3. Hypernuclear Spectroscopy in Hall $\mathrm{A}$ : The elementary reaction ${ }^{1} \mathrm{H}\left(e, e^{\prime} K^{+}\right) \Lambda, \Sigma^{0}$.

A. Acha, F. Cusanno, ... et al., contributed talk at the Hall A Collaboration Meeting, Jefferson Lab (Thomas Jefferson Accelerator Facility), Newport News, Virginia, 1314 Dec 2007.

4. High Resolution 1P-Shell Hypernuclear Spectroscopy at JLAB, HALL A (E94-107) A. Acha, P. Markowitz, ... et al., contributed talk at the $73^{\text {rd }}$ Annual Meeting of the Southeastern Section of the APS (SESAPS), Williamsburg, Virginia, 9-11 Nov 2006.

5. High Resolution 1p-shell Hypernuclear Spectroscopy at JLab, Hall A (E94-107) A. Acha, ... et al., Southeastern University Research Association (SURA) Poster Presentation, Users Group Workshop and Annual Meeting 2006, Jefferson Lab (TJNAF), Newport News, Virginia, 12-14 Jun 2006.

6. Particle Identification for JLab Experiment E94-107. A. Acha, P. Markowitz, ... et al., contributed talk at the $72^{\text {nd }}$ Annual Meeting of the Southeastern Section of the APS (SESAPS), Gainesville, Florida, 10-12 Nov 2005. 